\title{
The SuperCam Instrument Suite on the Mars 2020 Rover: Science Objectives and Mast-Unit Description
}

\author{
S. Maurice ${ }^{1}$ - R.C. Wiens ${ }^{2}$ - P. Bernardi ${ }^{3}$ P. Caïs ${ }^{4}$ S. Robinson ${ }^{2}$ - T. Nelson ${ }^{2}$.
}

O. Gasnault ${ }^{1} \cdot$ J.-M. Reess ${ }^{3}$ - M. Deleuze ${ }^{5}$ - F. Rull ${ }^{6}$ - J.-A. Manrique ${ }^{6} \cdot$ S. Abbaki ${ }^{7}$.

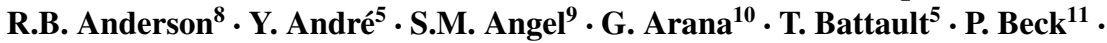
K. Benzerara ${ }^{12}$ - S. Bernard ${ }^{12}$ - J.-P. Berthias ${ }^{5}$. O. Beyssac ${ }^{12}$ - M. Bonafous ${ }^{3}$. B. Bousquet ${ }^{13}$ - M. Boutillier ${ }^{5}$ - A. Cadu $^{14}$ - K. Castro ${ }^{10}$ - F. Chapron ${ }^{3}$ - B. Chide Chit, $^{1,}$ K. Clark ${ }^{15}$ - E. Clavé ${ }^{13}$ - S. Clegg ${ }^{1}$ - E. Cloutis ${ }^{16}$ - C. Collin ${ }^{3}$ - E.C. Cordoba ${ }^{15}$. A. Cousin ${ }^{1}$ • J.-C. Dameury ${ }^{5}$. W. D'Anna ${ }^{4}$. Y. Daydou ${ }^{1}$ - A. Debus ${ }^{5}$ - L. Deflores ${ }^{15}$. E. Dehouck ${ }^{17}$ - D. Delapp ${ }^{2}$ - G. De Los Santos ${ }^{15}$. C. Donny ${ }^{5}$ A. Doressoundiram ${ }^{3}$. G. Dromart ${ }^{17}$ - B. Dubois ${ }^{18}$ - A. Dufour ${ }^{5}$ - M. Dupieux ${ }^{1}$ - M. Egan ${ }^{19} \cdot$ J. Ervin $^{15}$. C. Fabre ${ }^{20}$ - A. Fau ${ }^{12}$ - W. Fischer ${ }^{21}$ - O. Forni ${ }^{1}$ - T. Fouchet ${ }^{3}$ - J. Frydenvang ${ }^{22}$. S. Gauffre ${ }^{4}$. M. Gauthier ${ }^{12}$ - V. Gharakanian ${ }^{15}$. O. Gilard ${ }^{5}$ - I. Gontijo ${ }^{15}$. R. Gonzalez • D. Granena ${ }^{5}$ J. Grotzinger ${ }^{21}$ - R. Hassen-Khodja ${ }^{7}$ M. Heim ${ }^{5}$. Y. Hello ${ }^{3}$ G. Hervet ${ }^{5}$ O. Humeau ${ }^{7}$ - X. Jacob ${ }^{23}$ - S. Jacquinod ${ }^{3}$ - J.R. Johnson ${ }^{24}$. D. Kouach ${ }^{18}$ - G. Lacombe L N. Lanza $^{2}$ - L. Lapauw ${ }^{7}$. J. Laserna ${ }^{25}$ • J. Lasue ${ }^{1}$. L. Le Deit ${ }^{26}$ - S. Le Mouélic ${ }^{26}$ - E. Le Comte ${ }^{1}$ - Q.-M. Lee ${ }^{1}$ - C. Legett IV . $^{2}$ R. Leveille ${ }^{27}$ - E. Lewin ${ }^{28}$ - C. Leyrat ${ }^{3}$ - G. Lopez-Reyes ${ }^{6}$ - R. Lorenz Le $^{24}$ B. Lucero ${ }^{2}$. J.M. Madariaga ${ }^{10}$ - S. Madsen ${ }^{15}$ - M. Madsen ${ }^{22}$ - N. Mangold ${ }^{26} \cdot$ F. Manni ${ }^{5}$. J.-F. Mariscal ${ }^{7}$ - J. Martinez-Frias ${ }^{29}$ - K. Mathieu ${ }^{5}$ - R. Mathon ${ }^{1}$ - K.P. McCabe . $^{2}$ T. McConnochie $^{30}$ - S.M. McLennan ${ }^{31}$ - J. Mekki ${ }^{5}$ N. Melikechi ${ }^{32}$ - P.-Y. Meslin ${ }^{1}$. Y. Micheau ${ }^{18}$ - Y. Michel ${ }^{5}$ - J.M. Michel' ${ }^{2}$ D. Mimoun ${ }^{14}$ - A. Misra ${ }^{19}$ - G. Montagnac ${ }^{17}$. C. Montaron ${ }^{7}$ - F. Montmessin ${ }^{7}$ - J. Moros ${ }^{25}$ - V. Mousset ${ }^{5}$. Y. Morizet $^{26}$. N. Murdoch ${ }^{14}$ - R.T. Newell ${ }^{2}$ H. Newsom ${ }^{33}$ - N. Nguyen Tuong ${ }^{3}$ A.M. Ollila ${ }^{2}$. G. Orttner ${ }^{1}$ - L. Oudda ${ }^{5}$ L. Pares ${ }^{1}$ - J. Parisot ${ }^{3}$. Y. Parot ${ }^{1} \cdot$ R. Pérez ${ }^{5}$ D. Pheav ${ }^{5}$. L. Picot ${ }^{5}$ - P. Pilleri ${ }^{1}$ - C. Pilorget ${ }^{34}$ - P. Pinet ${ }^{1}$ - G. Pont ${ }^{5}$ - F. Poulet P $^{34}$. C. Quantin-Nataf ${ }^{17}$ - B. Quertier ${ }^{4}$. D. Rambaud ${ }^{1}$. W. Rapin ${ }^{12}$ - P. Romano ${ }^{15}$.

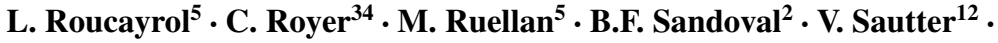
M.J. Schoppers ${ }^{15}$ - S. Schröder ${ }^{35}$ - H.-C. Seran ${ }^{1}$ - S.K. Sharma ${ }^{19}$ • P. Sobron ${ }^{36}$. M. Sodki ${ }^{5}$ A. Sournac ${ }^{14}$ - V. Sridhar ${ }^{15}$ - D. Standarovsky ${ }^{5}$ - S. Storms ${ }^{2}$ - N. Striebig ${ }^{18}$.

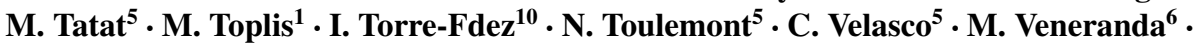
D. Venhaus ${ }^{2}$ - C. Virmontois ${ }^{5}$ - M. Viso ${ }^{5}$ - P. Willis ${ }^{15} \cdot$ K.W. Wong ${ }^{1}$

Received: 22 May 2020 / Accepted: 13 February 2021 / Published online: 12 April 2021

(C) The Author(s) 2021

The Mars 2020 Mission

Edited by Kenneth A. Farley, Kenneth H. Williford and Kathryn M. Stack

S. Maurice

sylvestre.maurice@irap.omp.eu

1 Institut de Recherche en Astrophysique et Planétologie, CNRS, Univ. Toulouse, CNES, Toulouse, France 


\begin{abstract}
On the NASA 2020 rover mission to Jezero crater, the remote determination of the texture, mineralogy and chemistry of rocks is essential to quickly and thoroughly characterize an area and to optimize the selection of samples for return to Earth. As part of the Perseverance payload, SuperCam is a suite of five techniques that provide critical and complementary observations via Laser-Induced Breakdown Spectroscopy (LIBS), Time-Resolved Raman and Luminescence (TRR/L), visible and near-infrared spectroscopy (VISIR), highresolution color imaging (RMI), and acoustic recording (MIC). SuperCam operates at remote distances, primarily 2-7 $\mathrm{m}$, while providing data at sub- $\mathrm{mm}$ to $\mathrm{mm}$ scales. We report on SuperCam's science objectives in the context of the Mars 2020 mission goals and ways the different techniques can address these questions. The instrument is made up of three separate subsystems: the Mast Unit is designed and built in France; the Body Unit is provided by the United States; the calibration target holder is contributed by Spain, and the targets themselves by the entire science team. This publication focuses on the design, development, and tests of the Mast Unit; companion papers describe the other units. The goal of this work is to provide an understanding of the technical choices made, the constraints that were imposed, and ultimately the validated performance of the flight model as it leaves Earth, and it will serve as the foundation for Mars operations and future processing of the data.
\end{abstract}

Keywords Mars 2020 PERSEVERANCE rover · Jezero crater · LIBS · Raman spectroscopy · Infrared spectroscopy · Microphone on Mars · Imaging on Mars

2 Los Alamos National Laboratory, Los Alamos, NM, USA

3 Laboratoire d'Etudes Spatiales et d'Instrumentation en Astrophysique, Observatoire de Paris, CNRS, Sorbonne Univ., Univ. Paris-Diderot, Meudon, France

4 Laboratoire d'Astrophysique de Bordeaux, CNRS, Univ. Bordeaux, Bordeaux, France

5 Centre National d'Etudes Spatiales, Toulouse, France

6 University of Valladolid, Valladolid, Espagne

7 Laboratoire Atmosphères, Milieux, Observations Spatiales, CNRS, Univ. Saint-Quentin-en-Yvelines, Sorbonne Univ., Guyancourt, France

8 U.S. Geological Survey, Flagstaff, AZ, USA

9 University of South Carolina, Columbia, SC, USA

10 University of Basque Country (UPV/EHU), Leioa, Bilbao, Spain

11 Institut de Planétologie et Astrophysique de Grenoble, CNRS, Univ. Grenoble Alpes, Grenoble, France

12 Institut de Minéralogie, de Physique des Matériaux et de Cosmochimie, Museum National d'Histoire Naturelle, CNRS, Sorbonne Université, Paris, France

Centre Lasers Intenses et Applications, CNRS, CEA, Univ. Bordeaux, Bordeaux, France

14 Institut Supérieur en Aéronautique et Espace, Toulouse, France

15 Jet Propulsion Laboratory/Caltech, Pasadena, CA, USA

16 University of Winnipeg, Winnipeg, Canada

17 Univ. Lyon, Univ. Lyon 1, ENSL, CNRS, LGL-TPE, Villeurbanne, 69007 Lyon, France

18 Observatoire Midi-Pyrénées, Univ. Toulouse, Toulouse, France 
Fig. 1 Mast-Unit investigations and overall objectives. LIBS: Laser Induced Breakdown Spectroscopy. TRR/L: Time Resolved Raman and Luminescence. VISIR: Visible and IR spectroscopy. RMI: Remote Micro Imaging. MIC: Microphone

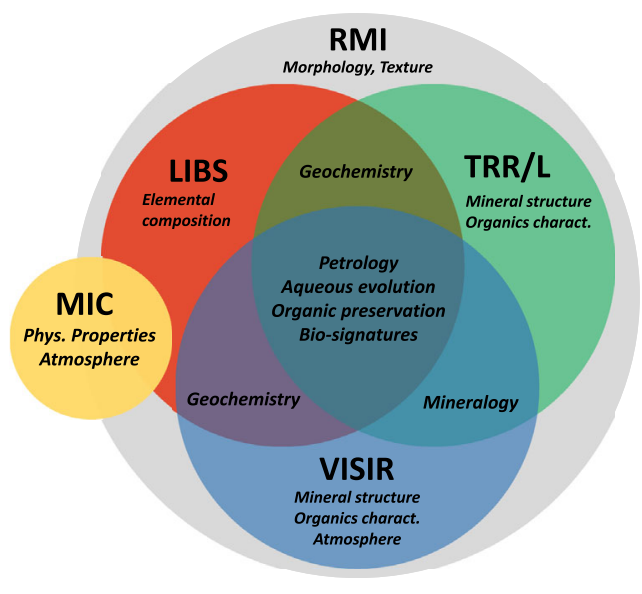

\section{Introduction}

On a mobile robotic mission to explore in-situ ancient sedimentary and aqueously altered deposits in a potentially habitable environment (Mustard et al. 2013; Williford, this journal), the remote determination of texture, mineralogy and chemistry of rocks along the traverse is essential to quickly and thoroughly characterize an area from which the best samples will be selected to return to Earth. This coordinated search will reveal the processes and environments responsible for the chemical alteration of primary igneous materials, their recycling as sedimentary rocks in an aqueous environment (e.g., in the form of clay and carbonate minerals) and the possible presence of organics. As part of the Mars 2020 Perseverance rover payload, SuperCam addresses these overarching goals with a suite of five co-boresighted investigations that provide key observations via Laser-Induced Breakdown Spectroscopy (LIBS), time-resolved Raman and Luminescence (TRR and TRL, or TRR/L combined), visible and near-infrared spectroscopy (VISIR), high-resolution color imaging (RMI), and sound recording with a microphone (MIC). The suite provides a highly synergistic analysis of rocks and soils. The greatest discoveries are likely to be made at the crossroads of the different techniques (Fig. 1).

19 University of Hawaii, Manoa, HI, USA

20 GéoRessources, CNRS, Univ. Lorraine, Nancy, France

21 California Institute of Technology, Pasadena, CA, USA

22 University of Copenhagen, Copenhagen, Denmark

23 Institut de mécanique des fluides de Toulouse, CNRS, INP, Univ. Toulouse, Toulouse, France

24 Applied Physics Laboratory, Johns Hopkins University, Laurel, MD, USA

25 Universidad de Malaga, Malaga, Spain

26 Laboratoire de Planétologie de Nantes, CNRS, Univ. Nantes, Nantes, France

27 McGill University, Montreal, Canada

28 Institut des Sciences de la Terre, CNRS, Univ. Grenoble Alpes, IRD, Univ. Savoie Mont Blanc, Grenoble, France

29 Agencia Estatal Consejo Superior de Investigaciones Científicas, Madrid, Spain 
SuperCam's telescope is accommodated at the top of the Remote Sensing Mast (RSM) so it can observe targets at remote distances. It complements the "proximity" instruments, PIXL and SHERLOC that operate within the arm workspace. Beyond the arm workspace, a circle of $\sim 7 \mathrm{~m}$ radius around the Perseverance rover is accessible to SuperCam optical investigations. This yields an observational area $>100 \mathrm{~m}^{2}$ by merely rotating the rover's mast. The RMI and VISIR investigations can also observe targets at longer distances, up to the crater walls (a few tens of kilometers away), depending on atmospheric opacity.

Along with these long-distance capabilities, SuperCam also operates at fine scales. Around the rover, the analytical footprint for LIBS and TRR/L is between $0.3 \mathrm{~mm}$ and $1 \mathrm{~cm}$ in diameter depending on the distance and technique. As shown later, this is at the scale of major features of interest for the mission, including mineral grains and rock textures. Several observation points on the same target may be necessary to explore its spatial heterogeneity. In this case, ground operators program "rasters", $N \times M$ sets of points on the same target, typically arranged as $1 \times 5,2 \times 2,3 \times 3$, and $1 \times 10$ matrices. Combining small investigation scales and large rasters, SuperCam returns a large quantity of information in relatively low data volume.

Most SuperCam observations are performed in less than 2 min per point, per investigation. The possibilities of combining the different investigations are numerous and provide the science team with many options for analyses. On average, it takes $\sim 40 \mathrm{~min}$ to collect data to fully characterize the chemistry and mineralogy of each target. Thanks to this rapidity, SuperCam is expected to study many targets, including several around each location where samples are selected for Earth-return to fully characterize the diversity of their geological context.

SuperCam benefits greatly from the heritage of ChemCam on Curiosity (Maurice et al. 2012; Wiens et al. 2012). In eight years of operations at Gale crater, Curiosity has acquired 800,000 LIBS spectra, many "VIS" spectra (called passive reflectance spectra), and 11,000 RMI images on > 3,200 different targets, which have all been returned to Earth. SuperCam possesses these same capabilities, except that the RMI images will be in color. The ChemCam and SuperCam instruments are very similar at first glance, but internally, almost every part has been redesigned to accommodate additional investigations (TRR/L, IR, and MIC).

The different investigation methods of SuperCam operate seamlessly and with techniques usually being coordinated successively for optimized sample analysis. Typically, LIBS will be performed prior to TRR, TRL, and VISIR spectroscopy to remove obscuring dust from the target. Moreover, when shooting the LIBS laser, the plasma expansion generates a shock wave, which both removes dust and can also be recorded with the SuperCam microphone, providing information on the physical properties of the target (Chide et al. 2019, 2020b).

This publication is the first of three which describe the SuperCam instrument and its expected performance for exploring Jezero crater, Mars. First, it introduces the SuperCam science objectives in the context of the Mars 2020 rover mission (Sect. 2). In a methodical

30 University of Maryland, College Park, MD, USA

31 State University of New York, Stony Brook, NY, 11794-2100, USA

32 University of Massachusetts, Lowell, MA, USA

33 University of New Mexico, Albuquerque, NM, USA

34 Institut d'Astrophysique Spatiale, CNRS, Univ. Paris-Sud, Orsay, France

35 Institute of Optical Sensor Systems, DLR, Berlin, Germany

36

SETI Institute, Mountain View, CA, USA 
way, these objectives lead to science requirements, then to an instrument concept that can be accommodated on the rover, and finally, an overall architecture (Sect. 3). This section highlights the three separate subsystems: the Mast Unit designed and built in France; the Body Unit designed and built in the US; the calibration target holder contributed primarily by Spain. The following section (Sect. 4) is devoted exclusively to the Mast Unit, including details of its design, development, and testing. The last section (Sect. 5) summarizes the Mast-Unit performance. Wiens et al. (this journal) focus on the Body-Unit design, and the performance of the whole instrument suite that has been measured prior to the launch. Manrique et al. (this journal) describe the selection of the calibration targets, their qualification, and integration on the rover. The fabrication and characterization of these targets is the subject of a dedicated publication.

\section{Scientific Context}

As a remote sensing suite, SuperCam responds broadly to the NASA solar system exploration goals and to all four of the Mars Exploration Program goals incorporated in the four objectives of the Mars 2020 mission (Farley et al., this journal):

A. Characterize the processes that formed and modified the geologic record within an astrobiologically relevant ancient environment,

B. Perform astrobiologically relevant investigations to determine habitability, search for materials with biosignature preservation potential, and search for evidence of past life,

C. Assemble a returnable cache of samples (including searching for the best samples and documenting their conditions), and

D. Contribute to preparation for human exploration of Mars (including characterization of atmospheric dust size and morphology).

Remote microscale characterization of the mineralogy and elemental chemistry of the Martian surface, along with the search for extant organic materials, lay the groundwork for several types of Mars geochemistry and astrobiology investigations. More specifically, we describe SuperCam science objectives and their relationship to the mission goals (see Table 1 for a qualitative representation of these relationships).

\subsection{Science Objectives}

We define eight Goals and describe how they fit into the mission objectives:

Goal 1: Rock Identification. SuperCam can identify specific minerals via TRR and VISIR spectroscopy, analyze major elements with LIBS, and identify some trace elements present in rocks with TRL. The high-resolution images provided by RMI (features < $80 \mu \mathrm{m}$ at $1 \mathrm{~m}$ ) in the context of broader Navcam (Maki et al., this journal) and Mastcam-Z (Bell et al., this journal) images complement these analyses by determining the rock type (e.g., sedimentary vs. igneous), its texture (e.g., porphyritic vs. aphanitic) and grain size distribution (down to medium sand grain size on the Wentworth scale). Quantification of the major elements on several points on each rock yields a smaller scale determination of the bulk chemistry as a complement to mineralogy. Conversely, the specific capability of TRR and VISIR to identify primary and secondary minerals (sulfates, carbonates, hydrous silicates, etc.) usefully completes the bulk chemistry provided by LIBS data. For instance, ChemCam has indirectly identified calcium sulfate from the presence of $\mathrm{S}, \mathrm{O}, \mathrm{H}$, and $\mathrm{Ca}$ in veins large 
enough for a LIBS spot, but it was challenging to determine the degree of hydration and the mineral phase to distinguish between bassanite and gypsum (Nachon et al. 2014; Rapin et al. 2016). TRR and VISIR spectra allow identification between these mineral phases in a more definitive way (Larkin 2011; Gaft et al. 2020). Additionally, long-distance RMI images coupled with SuperCam VISIR allow the long-range identification of key targets for broad geological interests, or tactical goals. An indication of rock hardness obtained with the microphone during LIBS analysis adds original information to help identify and characterize rocks.

As described above, SuperCam's detailed mineral, chemical and textural characterization of rocks along the rover traverse provides a broad dataset for determining the geological diversity of the site and for identifying key processes relevant to its aqueous history (Mission goal A) and for documenting the context of the sample cache (Mission goal C).

Goal 2: Sedimentary Stratigraphy and Facies/Alteration Characterization. Color images allow a detailed analysis of the sediment facies, thus providing identification of depositional features (ripples, cross-bedding, lamination, etc.) as well as diagenetic features (nodules, veins, etc.). The LIBS chemistry (including relevant minor and trace elements) (Ollila et al. 2014; Payré et al. 2017) and the mineralogy obtained by TRR and VISIR enable us to understand the provenance of the sediments, as well as any later modification by aqueous processes (e.g., diagenesis or weathering). More specifically, TRR discriminates secondary minerals that have chemistries close to that of more abundant primary minerals (e.g., zeolites vs. anhydrous silicates; quartz vs amorphous silica or chert in a hydrothermal area), or minerals that are not abundant enough to be detected from a variation in chemistry (e.g., goethite in Fe-rich sediments). For instance, ChemCam identified a hydrated cement binding fluvial conglomerate (Williams et al. 2013) without determining its mineralogy. As TRR can identify many hydrated minerals, the mineralogy of rocks like these should be readily identifiable with SuperCam.

Hence SuperCam's characterization of the texture and composition of the aqueous alteration and sedimentary structures (e.g., cross-bedding, lamination) provides strong constraints on the aqueous processes (Mission goal A) as well as the landing site potential habitability (Mission goal B).

Goal 3: Organics and Bio-signatures. LIBS data allow the identification of light elements that are the major building blocks of organic molecules, such as $\mathrm{C}, \mathrm{H}, \mathrm{N}, \mathrm{O}, \mathrm{P}$, and $\mathrm{S}$ at various levels of detection (Wiens and Maurice 2015; Maurice et al. 2016). LIBS may also identify elements such as Mn that, when concentrated, are associated with biological activity on Earth (Lanza et al. 2016), or other elements necessary for life (e.g., Gasda et al. 2017). This capability coupled with TRR, and especially short-lived luminescence revealed by TRL, enables the identification of a large number of organic molecules - if they are present in sufficient concentration - from the variety of molecular vibrations between $\mathrm{C}, \mathrm{N}, \mathrm{O}$ and $\mathrm{H}$. The signal is enhanced for conjugated organic structures. The comparison between LIBS and TRR allows us to determine if $\mathrm{C}, \mathrm{N}$, and $\mathrm{H}$ detected by LIBS are associated with minerals, as opposed to $\mathrm{CO}_{2}$, and $\mathrm{N}_{2}$ atmospheric contamination, or adsorbed $\mathrm{H}_{2} \mathrm{O}$. If present in sufficient concentration, aliphatic organic molecules may be identifiable in VISIR (Cruikshank et al. 2019). The observation of organic molecules on specific textured surfaces at the scale of the Wide-Angle Topographic Sensor for Operations and eNgineering (WATSON) imager (Bhartia et al., this journal) facilitates the detection of potential bio-signatures. 
Hence, SuperCam analyzes astrobiologically relevant materials (Mission goal B) without requiring contact, determines the best area for proximity science and caching (Mission goal C), and allows interrogation of areas inaccessible to the rover arm.

Goal 4: Volatiles (Hydration and Halogens). Among the volatiles detectable by LIBS, H is key to determine aqueous alteration, not only in primary hydrous phases but also as cements or secondary hydrated phases, such as sulfates and phyllosilicates. Hydrogen, as well as many other light elements such as $\mathrm{S}, \mathrm{Cl}, \mathrm{F}, \mathrm{P}$, and $\mathrm{Li}$ are quantifiable by LIBS (Forni et al. 2015; Schröder et al. 2015; Payré et al. 2017; Rapin et al. 2017; Anderson et al. 2017); they may be observed in igneous rocks, where they serve as indicators of volatile processes in the crust, as well as in sedimentary rocks, where they can provide evidence of fluid circulation. TRR and VISIR enable the discrimination of the phases in which volatiles are present. TRR is a powerful method to investigate the speciation of $\mathrm{OH} / \mathrm{H}_{2} \mathrm{O}$ structurally present in minerals (Bishop 2019; Sharma and Egan 2019).

Hence, SuperCam will constrain the aqueous processes involving volatiles (Mission goal A \& B) and provide data on volatile content for the documentation of cached material (Mission goal C).

Goal 5: Context Morphology and Texture. Thanks to its high resolution, RMI can be used as an independent tool for analyzing specific outcrop geometries, such as the presence of bedding or contacts between units. Layered facies can be analyzed over scales of a few $\mathrm{mm}$ at distances up to several hundred meters away from the rover. Images taken within $7 \mathrm{~m}$ can also be used to analyze features that are potentially unrelated to the underlying nature of rocks or to identify modifications to the initial rock texture, such as wind abrasion or open cracks, which can potentially links to recent or current environmental conditions (wind direction, frost action, etc.) (Bridges et al. 2014; Le Mouélic et al. 2015; Mangold et al. 2017). Imaging will also provide information about the color of mineral phases, which can greatly aid in the interpretation of their chemistries and potentially their redox states, which in turn can provide key insight into past aqueous environments.

Hence, high resolution color images will provide detailed information on dust coverings (Mission goal D2) and a visual approach to oxidation states of layers relevant for aqueous processes (Mission goal A).

Goal 6: Coatings and Varnishes. Chemical weathering may modify rock compositions by either adding or removing material through limited aqueous alteration that can produce coatings and rinds. LIBS is uniquely capable for remotely identifying coatings by detecting coatings enriched in trace elements (Ollila et al. 2014) or minor elements such as Mn, especially by providing depth profiles (up to 500 laser shots at once) at the scale of the coating thickness $(\sim 10-100 \mu \mathrm{m})$ (Lanza et al. 2015). On Earth, rock coatings can be a key indicator for the presence of past or extant life because many are produced by microbes or colonized by them (Parchert et al. 2012; Northup et al. 2010), and such communities may leave behind organic material that may be preserved even after they are no longer living. The coupling of TRR/L analysis will provide a strong constraint on detection and possibly characterization of any organicbearing coatings (Misra et al. 2016). VISIR will complement these observations by identifying areas in which to search for coatings that are distinct from country rocks. The microphone will help to determine the depth of the transition between the coating and the underlying rock (Lanza et al. 2020).

Hence, SuperCam analyses of coatings will allow the identification of late-stage weathering (Mission goal A) and its relationships to potential exobiological material (Mission goal B). 
Goal 7: Regolith Characterization. Regoliths and soils constitute a record of the alteration processes that transformed the igneous Martian crust, or result from impact processes. As such they reflect the evolution of climate and habitability conditions. With its subcentimeter resolution, SuperCam may characterize the soil's chemical and mineral heterogeneity, including its abundant amorphous components, and will relate it to grain size, in order to better understand the respective contributions from local and global sources (Meslin et al. 2013; Cousin et al. 2017; David et al. 2020). Aeolian dust composition may be analyzed by LIBS at the surface of rocks and onboard calibration targets to assess its origin (Lasue et al. 2018). Additionally, hydrogen characterization by LIBS provides a tool for analyzing amorphous phases and adsorbed water (Meslin et al. 2013; Schröder et al. 2015; David et al. 2020), while the presence of brines may be assessed by Raman spectroscopy (Zhang and Chan 2003). Coupling of VISIR with this $\mathrm{H}$ analysis provides a strong constraint to the unsolved question of the origin of widespread hydration at Mars' surface.

Thus, SuperCam addresses soil diversity at the landing site (Mission Goal A) and can characterize the soil potential for biosignature preservation (Mission Goal B2).

Goal 8: Atmospheric Characterization. The $\mathrm{CO}, \mathrm{O}_{2}$, and $\mathrm{H}_{2} \mathrm{O}$ relative abundances and distributions are controlled, in large part, by odd hydrogen radicals sourced from photolyzed water vapor. They have been observed individually but have never been observed simultaneously in the same atmospheric column before, as proposed by $\mathrm{Su}-$ perCam (Nair et al. 1994; McConnochie et al. 2018). Atmospheric CO, $\mathrm{O}_{2}$, and $\mathrm{H}_{2} \mathrm{O}$ are all potentially valuable for in-situ resource utilization (Meurisse and Carpenter 2020); $\mathrm{O}_{2}$ variability and local-scale $\mathrm{H}_{2} \mathrm{O}$ temporal variability are poorly known and are affected by synoptic and global atmospheric circulation, sources and sinks. Additionally, the microphone can directly sample many atmospheric phenomena, such as wind (Chide et al. 2020a), vortices or dust devils, etc. (e.g., Williams 2001).

Thus, SuperCam will measure atmospheric molecules, water ice, and dust characteristics, which address the radiative balance of the atmosphere, as well as observing atmospheric phenomena, which will prepare for human exploration (Mission goal D2, D3).

As described above, SuperCam covers the majority of Mars 2020 science objectives. SuperCam's measurements are conducted from the top of the mast to cover the arm work zone and its geological surroundings further afield. To this end, all investigations operate at least between $2 \mathrm{~m}$ and $7 \mathrm{~m}$ (and imaging and passive spectroscopy can perform at distances as far as atmospheric transparency allows). Over this distance range, the sampled footprints are very small: between $300 \mu \mathrm{m}$ and $600 \mu \mathrm{m}$ for LIBS spots, between $1.5 \mathrm{~mm}$ and $8 \mathrm{~mm}$ for the TRR/L and IR spectroscopy, from $160 \mu \mathrm{m}$ to $560 \mu \mathrm{m}$ for the RMI resolution (size of the smallest detail that can be detected, given by the point spread function which covers $4 \times 4$ white pixels - each white pixel is the assembly of 1 red, 2 green, and 1 blue color pixels). The size of the LIBS footprints as well as the resolution of RMI images are the same as on ChemCam. Figure 2 shows the extent to which the size of these analyses is consistent with the typical dimensions of the essential geological features including grain size, diagenetic features, bio-structures, and sedimentary facies. To study targets with sizes similar to the investigation footprint, "fine scale" pointing is required with possible several iterations with the ground operations. To examine the heterogeneity of targets with sizes larger than the investigation footprints, multi-point targeting is possible, namely "raster" or "scan" modes (see Wiens et al. (this journal) for details).

Importantly, SuperCam can analyze diversity in composition of any rock type at millimeter scales. For instance, the individual mineral composition of igneous porphyritic minerals 

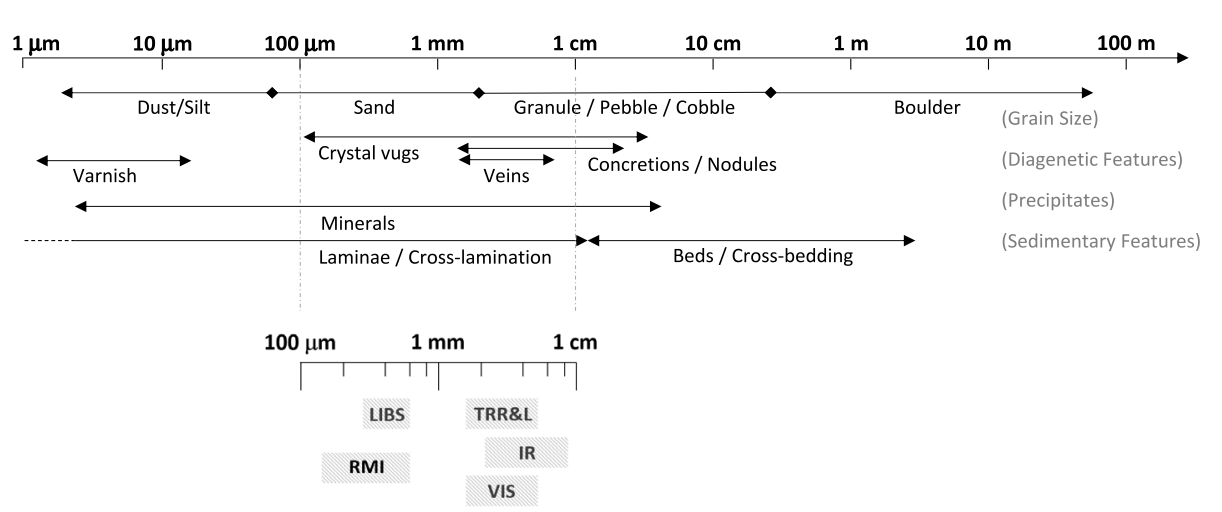

Fig. 2 Typical spatial scales of geologic features of interest to the Mars 2020 mission. At the bottom, the footprints of the different analytical investigations between $2 \mathrm{~m}$ and $7 \mathrm{~m}$ to targets. The RMI bar refers to the dimension of the smallest resolvable feature. The RMI field of view itself ranges from $3.8 \mathrm{~cm}$ to $13.3 \mathrm{~cm}$ over this distance range

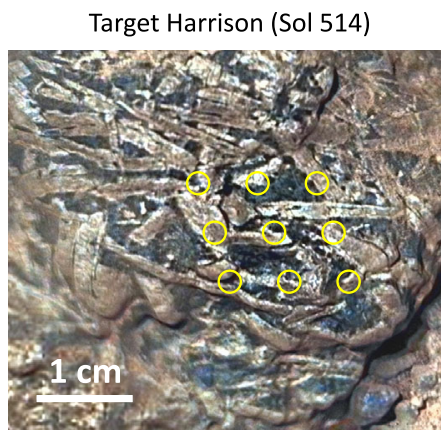

Target Howey (Sol 353)

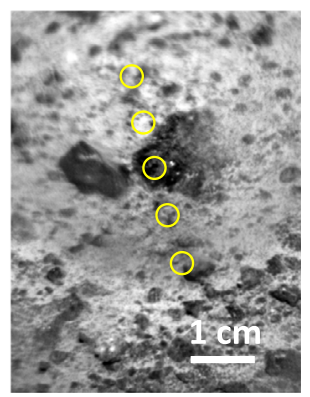

Target Cumberland (Sol 283)

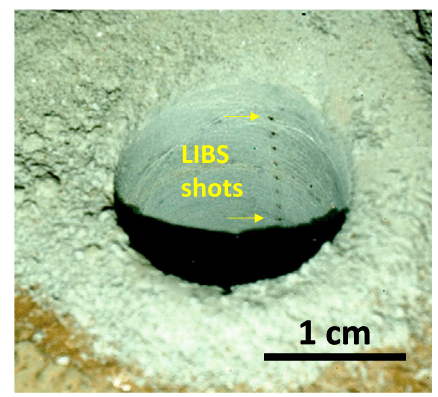

Fig. 3 Illustrations of SuperCam's capability to investigate various rock types at sub-centimeter scales from Curiosity data. (Left) ChemCam/Mastcam composite of a magmatic clast with elongated, light-toned crystals determined as feldspars. (Middle) ChemCam RMI image of a conglomerate with varying grain types that can be assessed individually. (Right) MAHLI image of the Cumberland drill hole that contains veins, with a raster of $\times 10$ LIBS bursts ( 30 shots each); only LIBS pits from bursts 3 (bottom arrow) to 10 (top arrow) are clearly visible (the two first points are in shadow). The sample contains detrital basaltic minerals, calcium sulfates, iron oxides, sulfides, and smectites

(Fig. 3, left), coarse sedimentary rocks such as conglomerates (Fig. 3, middle), and diagenetic veins (Fig. 3, right) was deduced indirectly from the elemental chemistry obtained by ChemCam (Sautter et al. 2015; Mangold et al. 2016; L'Haridon et al. 2020). On such similar objects, SuperCam TRR and VISIR will enable the direct and more definitive determination of the mineralogy, in addition to the elemental chemistry provided by LIBS on local points. Variable rasters of $3 \times 3$ or $1 \times 5$ points (as in Fig. 3, left and middle) will enable a statistical determination of the major element bulk chemistry. These capabilities will be helpful in determining the provenance of sediments when analyzing the sedimentary rocks of the delta fan in Jezero crater, or when assessing the diversity in crustal rock types on the crater rim in order to sample those that are the most representative samples. The capability to analyze the inner wall of the drill hole is unique to SuperCam and SHERLOC. 


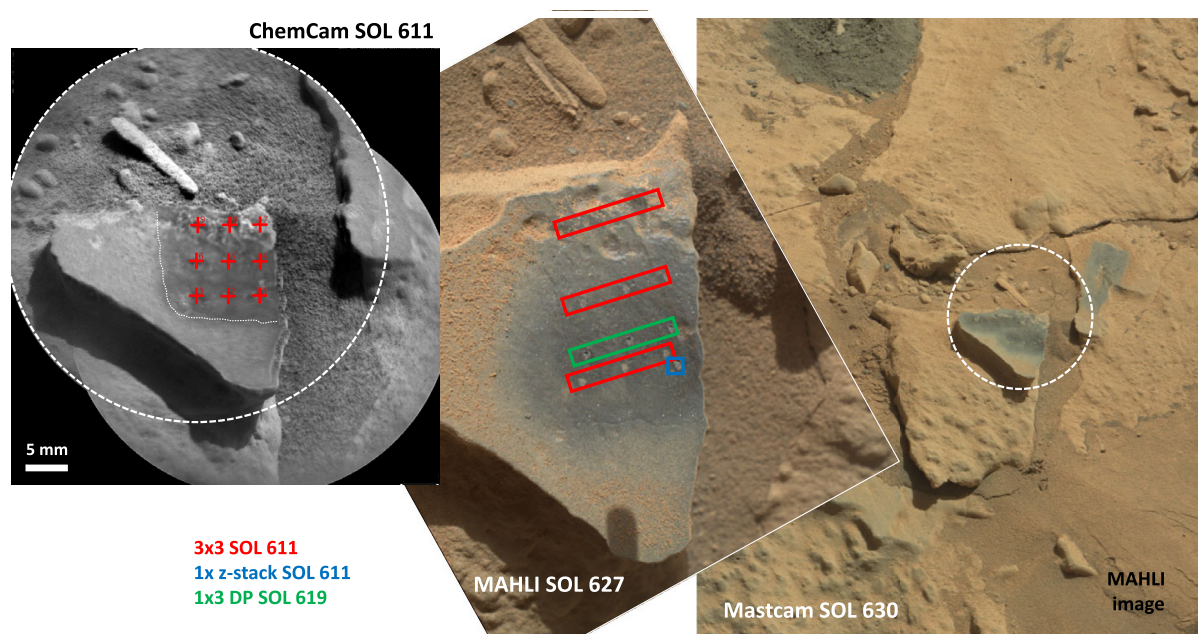

Fig. 4 Dust removal by LIBS based on ChemCam examples. Study of Stephen rock (2.25 m) with LIBS activities: $3 \times 3$ raster on Sol 611, $1 \times$ z-stack on Sol 611, $1 \times 3$ depth profile on Sol 619, $1 \times 5$ depth profile on Sol 630. (Left) ChemCam RMI after the first $3 \times 3$ raster. A thin dashed line delineates the region that has been cleared from dust. The thick circle indicates the RMI field of view. (Middle) Same rock as seen by MAHLI on Sol 627. The sub-millimeter LIBS pits are clearly visible. (Right) Broader view by MastCam on Sol 630 after the last LIBS activity

The ability of LIBS to blow off dust is a key SuperCam capability that enhances science return from its multiple measurement techniques. Owing to the much smaller size of the LIBS laser impacts compared to the analytical footprint of TRR/L, LIBS laser shots are expected to have no noticeable effects on Raman spectra (Fau et al. 2019). Figure 4 illustrates the efficiency of this process. At Gale crater, a rock of a few centimeters, Stephen, was studied in detail by ChemCam: a $3 \times 3$ raster of 30 shots/point on Mars solar day (Sol) 611 , an 8-burst z-stack (30 shots each, different focus) on Sol 611, a $1 \times 3$ depth profile of 150 shots/point on Sol 619, and a $1 \times 5$ depth profile of 150 shots each on Sol 630. After LIBS activities on Sol 611, an RMI shows that dust has been blown away over a few $\mathrm{cm}^{2}$ to reveal a darker veneer that turned out to be Mn-rich (Lanza et al. 2016). The images in Fig. 4 show how well the dust was cleared from this surface.

To summarize, SuperCam's objective is to analyze targets within their geological context, with no sample preparation (aside from potentially removing dust using LIBS). Multi-point analyses and statistical surveys are obtained at sub-centimeter scales. Since SuperCam is relatively simple to use (low in resource use) and highly relevant to the mission objectives, as is ChemCam on Curiosity, we expect to use it daily either by itself or, most of the time, as part of a broader observation strategy in association with the other remote sensing instruments of the payload.

\subsection{SuperCam Investigations}

SuperCam is a very versatile instrument, with four complementary spectroscopic techniques, an imager and a microphone. In order to optimize the instrument design, to understand how environmental factors can affect the properties or performance of the instrument and subsystems, and ultimately to obtain better quality data, it is essential to understand some details of the physics of those techniques. This physics is at the atomic, molecular 
or crystal scale and is summarized hereafter for LIBS, Time-Resolved Raman (TRR) and Luminescence (TRL), and VISIR spectroscopy. Some of these techniques have much in common: TRR and TRL occur nearly simultaneously, though slight differences in response times can be exploited by time-resolved measurements. TRR and VISIR spectroscopy are non-destructive and highly complementary techniques, providing independent information on the mineralogy of the target. The physics of the LIBS plasma formation and evolution also explains the origin of the sounds that can be captured by the SuperCam microphone. The description of each technique is followed by a discussion of the elemental or mineralogical information that can be acquired. The performance requirements follow after. For completeness, we first briefly introduce imaging (RMI) and sound measurements (MIC).

\subsubsection{Remote Micro-Imaging (RMI)}

SuperCam carries a three-color imager with a small field of view to document the various spectral analyses. There have been numerous color imagers on previous NASA rovers, and more to come on the US Perseverance, Chinese Tianwen-1, and European ExoMars/Rosalind Franklin rovers. SuperCam's RMI is an advanced color imager with a field of view that includes the analysis spots of the spectroscopic techniques. ChemCam's panchromatic RMI on Curiosity provided the highest resolution images of the rover remote sensing cameras (Le Mouélic et al. 2015). The situation is similar on Perseverance, with SuperCam RMI providing the highest resolution of remote sensing cameras, but also the smallest field of view. SuperCam RMI should therefore be highly complementary to Mastcam-Z investigations. While keeping a similar pixel resolution compared to ChemCam's RMI, SuperCam's RMI is upgraded by providing a color capability.

\subsubsection{Sound Recording (MIC)}

At the top of the rover mast, SuperCam records sounds between $100 \mathrm{~Hz}$ and $10 \mathrm{kHz}$, from natural and artificial sources, including laser-induced sounds related to LIBS. On two previous occasions, a microphone was developed for the surface of Mars (Delory et al. 2007): on the Polar Lander, which did not reach the surface of Mars safely, and on the Phoenix lander, where it was not switched on to avoid a major electrical interference with a high-priority instrument. SuperCam uses the same commercial microphone as flown before on these two missions. On top of atmospheric and artificial background sounds, its originality is to record the shock wave produced by LIBS sparks. Hence, it can help characterize rock hardness and other physical properties (Murdoch et al. 2019; Chide et al. 2019, 2020b).

\subsubsection{Laser Induced Breakdown Spectroscopy (LIBS)}

ChemCam on Curiosity was the first LIBS experiment in space (Wiens and Maurice 2015; Maurice et al. 2016). A similar instrument, MarSCoDe, is installed on the Chinese Tianwen-1 rover that is to land on Mars the same year as Perseverance (Xin et al. 2018). SuperCam's LIBS characteristics are very similar to those of ChemCam, with the added capability to perform time-resolved acquisitions for the spectral range of 536-900 nm. The physics of LIBS is described below.

The breakdown processes that result in the creation of a LIBS plasma depend on laser characteristics such as the laser pulse length and its wavelength as well as properties of the sample itself, such as its optical absorption at the laser wavelength, its thermodynamic properties, and the pressure of the ambient gas above its surface (e.g. Miziolek et al. 2006). When 
the leading edge of a nanosecond laser pulse interacts with sufficient irradiance with the surface of a target, sample material is heated, melted and vaporized (Anabitarte et al. 2012; Fau et al. 2019). Thermal effects dominate the ionization process for nanosecond lasers. A micro plasma forms and expands at supersonic speed, which produces an audible sound in an ambient gas (e.g. Cremers and Radziemski 2006). The plasma plume is a mixture of electrons, atoms, ions, and molecules from both vaporized sample material and ambient gas. The shockwave expansion can be described by the Taylor-Sedov model (Taylor 1950).

Between 1 and 10 nanoseconds after the start of the irradiation, in typical laboratory experiments the plasma becomes opaque to laser radiation. Therefore, the last part of the nanosecond laser pulse interacts with the expanding plasma front and is partially absorbed or reflected. At this point, the efficiency of the ablation decreases. This effect is called plasma shielding and is strongly dependent on environmental conditions (surrounding gases or vacuum) and experimental conditions (laser irradiance and wavelength). Martian atmospheric pressure is close to ideal for LIBS due to an optimal configuration of relatively high ablation rate at lower pressures and plasma heating due to plasma shielding that leads to stronger plasma emission (Brennetot et al. 2003).

After ignition, the plasma expands and cools; the electron temperature and density decrease. As the plasma cools, ions recombine with free electrons to form neutral atoms under the so-called radiative recombination. Free electrons decelerate when interacting with atomic nuclei under the free-free transition process called thermal bremsstrahlung. Both mechanisms produce a broad continuum of electromagnetic emission. In addition, the ionic and neutral de-excitation processes exhibit a discrete (or quantized) set of energy levels with related emission lines characteristic of each element, allowing their identification. Molecular emission of simple diatomic molecules occurs as well due to the recombination of plasma species in colder plasma regions. In Martian atmospheric conditions, the maximum intensity of these molecular bands is observed to last slightly later than that of ionic and atomic emission and stays intense for a longer period of time due to the low-temperature dependence (Vogt et al. 2018). If the plasma emission is collected over the entire plasma lifetime, the continuum signal can simultaneously exhibit atomic emission lines, and sometimes molecular bands from simple molecules. If the acquisition can be delayed by a few microseconds possible on SuperCam's transmission spectrometer, see Wiens et al. (this journal), some molecular lines can be isolated.

The discrete emission lines characterize the elemental composition: they are readily identifiable since their position is fixed in wavelength. To derive quantitative elemental compositions, the LIBS data can be analyzed with calibration models, where measurements are compared to those of samples whose composition is known, measured in laboratory-simulated Martian atmospheric conditions. On this basis, several univariate and statistical methods have been developed over the years to increase the accuracy of quantitative analysis and to overcome matrix effects in particular for the Martian LIBS data (Wiens et al. 2013; Clegg et al. 2017).

The implementation and data analysis of LIBS on SuperCam is similar to how it is done on ChemCam (Maurice et al. 2016). SuperCam yields quantitative elemental compositions for all major rock-forming oxides, $\mathrm{SiO}_{2}, \mathrm{TiO}_{2}, \mathrm{Al}_{2} \mathrm{O}_{3}, \mathrm{FeO}_{\mathrm{T}}, \mathrm{MgO}, \mathrm{CaO}, \mathrm{Na}_{2} \mathrm{O}$, and $\mathrm{K}_{2} \mathrm{O}$, with associated uncertainties. SuperCam also quantifies $\mathrm{H}$ by univariate analysis (Rapin et al. 2017), and can measure the non-metallic elements C, N, O, P, and S, despite degraded detection limits for $\mathrm{C}$ and $\mathrm{O}$ in rocks analyzed under Martian conditions (i.e. $\mathrm{CO}_{2}$ atmosphere). $\mathrm{F}$ and $\mathrm{Cl}$ are indirectly measured through the molecule emissions from secondary Ca-bearing species $\mathrm{CaF}$ and $\mathrm{CaCl}$, or through the $\mathrm{Cl}$ neutral line at $837.8 \mathrm{~nm}$ (Forni et al. 2015). Other measured elements are Li, B, Cr, V, Mn, Ni, Cu, Rb, Sr, Ba, and Zn. Quantification is obtained using comprehensive ground reference datasets, which are chosen to 
Fig. 5 Simplified Jablonski diagram for the different transitions responsible for the IR, Raman, and luminescence signals. The anti-Stokes emission is shown for reference but not used by SuperCam

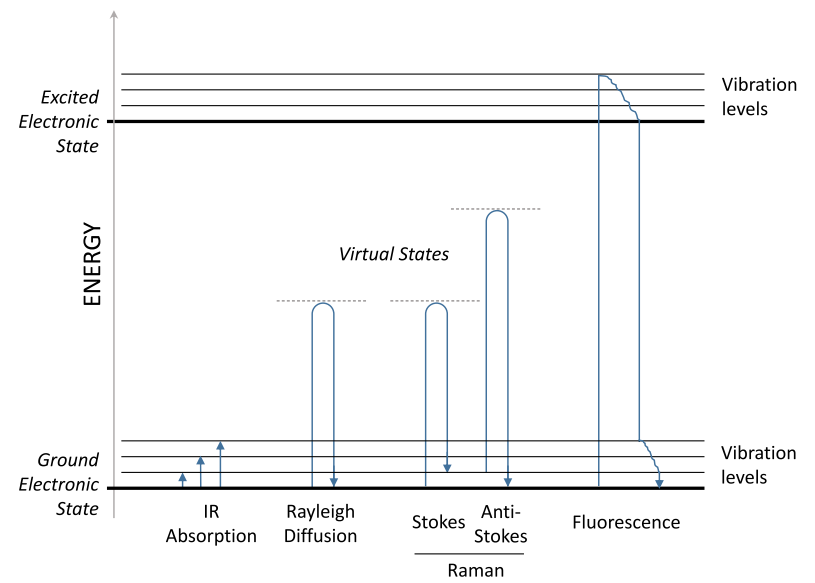

mimic the expected mineralogy and chemistry on Mars (Ollila et al. 2014; Payré et al. 2017; Gasda et al. 2017; Lanza et al. 2016).

It is worth noting that at even longer delays $\sim 75-100 \mu \mathrm{s}$, when the plasma is relatively cold and atomic, ionic, and molecular emissions have already quenched, other types of radiation may occur within matrices containing luminescence centers. The plasma plume acts as the excitation source in this case; the UV-VIS photons from the plasma activate the luminescence centers (Plasma Induced Luminescence). Such luminescence usually has long decay times, as for trivalent rare earth elements $\left(\mathrm{REE}^{3+}\right), \mathrm{Cr}^{3+}, \mathrm{Fe}^{3+}$, and $\mathrm{Mn}^{2+}$ in minerals (Gaft et al. 2019).

\subsubsection{Time Resolved Raman (TRR)}

SuperCam is the first stand-off Raman and luminescence spectrometer in space. Both Raman and luminescence measurements will also be made by SHERLOC at micron scales in close proximity to the target using a UV laser (Bhartia et al., this journal) to specifically detect and analyze organic molecules. Other instruments based on continuous wavelength lasers will fly on ExoMars and MMX rovers (Rull et al. 2017; Schröder et al. 2020). The physics of Raman and luminescence are briefly described below and in the following section, respectively.

Raman spectroscopy is a vibrational spectroscopy technique based on a scattering process widely used in chemistry, material sciences and mineralogy to characterize the vibrational state of materials. This provides a structural fingerprint by which such molecules, or crystals can be identified (e.g. Dubessy et al. 2012; Beyssac 2020; Pasteris and Beyssac 2020, and references therein).

For Raman spectroscopy, SuperCam uses visible light $(532 \mathrm{~nm})$ that cannot be absorbed through phonon excitation as in the case of infrared spectroscopy. This is because the laser photon energy is much greater than the energy difference between the vibrational states of the molecules. Rather, visible light is scattered and excites the molecules to virtual energy states with extremely short lifetimes and quasi-immediate relaxation (Fig. 5). Most light scattering is essentially elastic (Rayleigh scattering), i.e., molecules end up in the same vibrational state as before excitation, and the energy of the scattered light is unchanged. But a very small fraction ( 1 in $10^{7}$ incident photons) of the scattering occurs inelastically. After scattering, molecules then reside at a higher (photon energy loss for the photon: Stokes scattering) or lower (photon energy gain for the photon: anti-Stokes scattering) vibrational 
state than before irradiation. Consequently, the photon energy of the scattered light is either decreased or increased with respect to the exciting photon: this is the Raman effect. Note that this photon energy difference corresponds to the specific energy difference between vibrational states of the molecule, thereby imparting molecular information to the Raman signal. In the case of minerals, the molecular vibration contributes to the Raman spectrum by generating not only internal modes but also lattice vibrations. Vibrations of atoms in a crystal structure are not independent and when atoms vibrate around their equilibrium position synchronously, they create fundamental lattice vibrations (phonons) accessible by Raman spectroscopy. Importantly, these lattice vibrations carry unique crystallographic information allowing the distinction between polymorphs.

SuperCam's Raman spectrometer differs from commercial laboratory instruments in two fundamental ways. First, SuperCam senses the Raman signal at large distances (i.e. 2 to $7 \mathrm{~m}$ ) with large laser spot sizes (i.e. several $\mathrm{mm}$ in diameter) while commercial laboratory instruments detect Raman signals at short distances $(\sim \mathrm{mm})$ with small spot sizes $(\sim \mu \mathrm{m})$ when using a microscope. Hence, SuperCam's Raman will likely probe a population of diverse mineral phases depending on grain size and effective spot diameter, while laboratory instruments generally target a single phase because their spot is smaller than the grain size. Second, SuperCam utilizes a pulsed excitation source combined with a gated detector, while commercial laboratory instruments typically employ continuous-wave $(\mathrm{CW})$ excitation sources and detectors. The idea is that the Raman signal (lifetime $\sim 10^{-15} \mathrm{~s}$ ) is only produced during the interaction of the sample with the excitation pulse, while other signals such as luminescence (lifetime $>1 \mathrm{~ns}$ ) start at the pulse but continue to decay after the excitation (Gaft et al. 2015). Using a short gate (100 ns for SuperCam) that is synchronized with the laser pulse, allows efficient rejection of the unwanted signals, like luminescence or daylight entering the telescope, and optimizes the collection of the Raman signal in the time domain. Time-resolved spectroscopy further allows collection of only the luminescence signal by opening the detector gate with delay after the laser pulse: by then the Raman signal is over while luminescence is still present (Beyssac 2020). Compared to CW Raman instruments, SuperCam Raman benefits from signal intensification and filtering in the time domain, but uses considerably less excitation, hence receives less signal because of the non-continuous excitation.

SuperCam records Stokes emissions between 0 and $4000 \mathrm{~cm}^{-1}$. However, the $0-150 \mathrm{~cm}^{-1}$ range is cut by filters blocking the Rayleigh-scattered laser light. Figure 6 is a simplified map of Raman peaks for major minerals and some organics. The main "fingerprint region" from which mineralogy and molecular structure are determined is in the $150-1500 \mathrm{~cm}^{-1}(536.3-578.1 \mathrm{~nm})$ range. In this window, internal modes (e.g. stretching, bending modes) from molecular groups (e.g. $\mathrm{CO}_{3}, \mathrm{PO}_{4}, \mathrm{SiO}_{4}$ ) present in minerals occur at higher wavenumbers than lattice vibrations. At higher wavenumbers, internal vibrations in $\mathrm{CH}\left(\sim 2900 \mathrm{~cm}^{-1}\right)$ from organics, or $\mathrm{OH}$ and $\mathrm{H}_{2} \mathrm{O}\left(\sim 3300-3700 \mathrm{~cm}^{-1}\right)$ present in minerals provide important information. Raman spectra collected under Martian surface temperature and pressure conditions yield the same spectral position (not necessarily FWHM) as those obtained in terrestrial laboratories, allowing existing Raman spectral databases to be used for mineral identification.

\subsubsection{Time-Resolved Luminescence (TRL)}

Time-resolved optical stimulation of luminescence has recently become established as an important method for mineral detection (Lisitsyn et al. 2011; Gaft and Panczer 2013; Chithambo 2018). When light interacts with a molecule and the energy of the incident photon gets close to the transition between two electronic states, the molecule energy shifts 


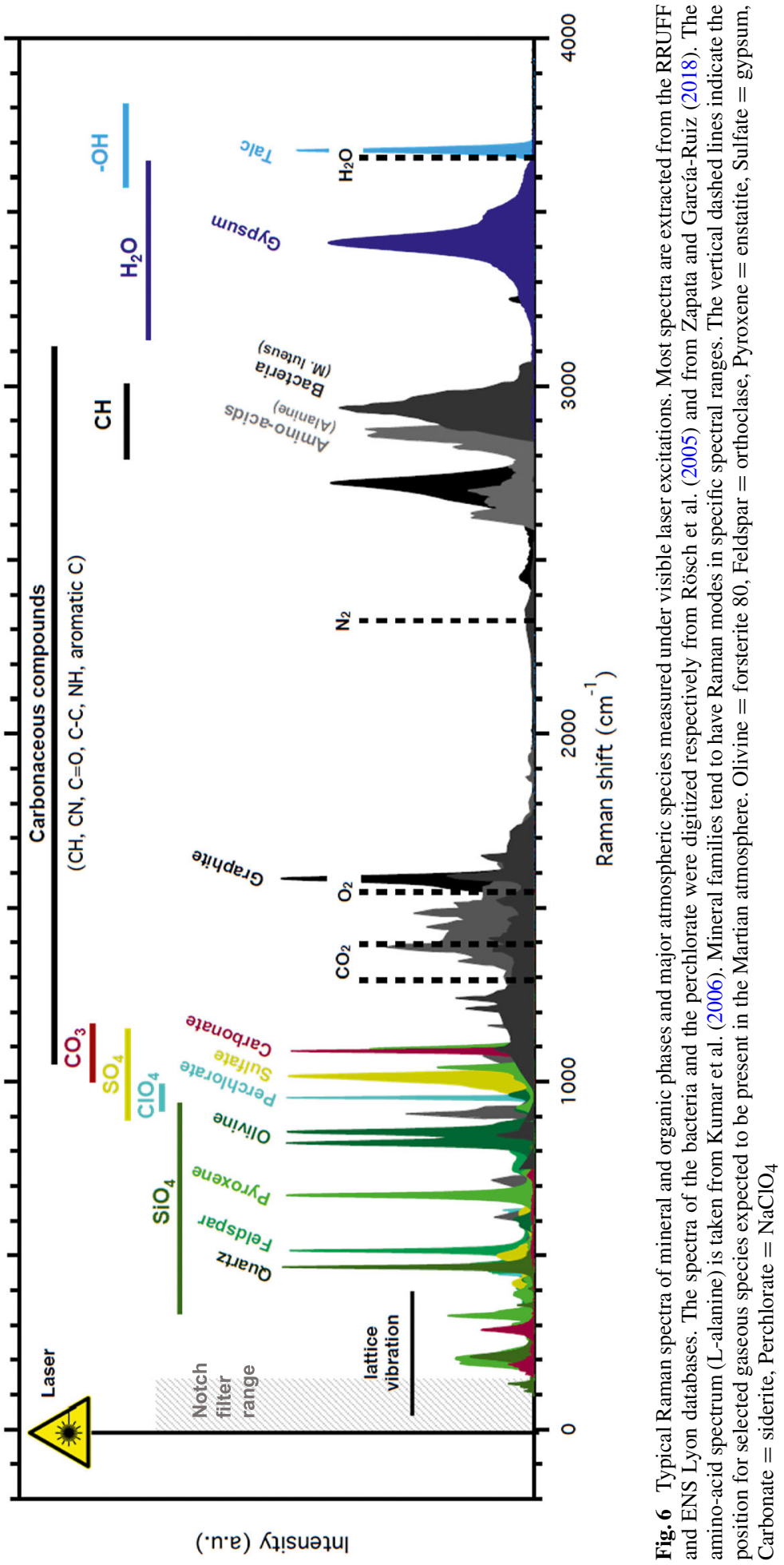


from its ground state to various vibrational states in the excited electronic state (Fig. 5). Following the absorption, the molecule's atoms adjust their relative positions to the new excited situation. During this process, called relaxation, there is usually no light emission. After some time (nanoseconds to milliseconds), the system returns spontaneously to the vibrational states of its ground electronic state, emitting a luminescence photon. The emission occurs at a lower energy than the absorption due to the relaxation process.

In addition, many mineral phases contain impurities (e.g., defects, trace elements, and organics) that can generate a luminescence signal through electronic transitions when excited by the laser (Gaft and Panczer 2013). This luminescence signal may consist of broad bands to a continuous background, as exhibited by $\mathrm{Mn}^{2+}$ substitution in calcite for example (Mason et al. 2005). Alternatively, emission spectra of luminescence may consist of narrow lines, small energy transitions, as exhibited by REEs in many minerals (Gaft et al. 2020). Luminescence may be so intense that, without the time resolution of SuperCam, the Raman signal becomes subsumed by, and often indistinguishable from, the luminescence band and/or background.

Luminescence is characterized by its wavelength distribution, quantum yield, and lifetime (Gaft and Panczer 2013). Quantum yield is the ratio of the number of photons emitted by luminescence to the number of photons absorbed, thus the probability of the excited state being deactivated by luminescence rather than by another non-radiative mechanism, such as the loss of energy in the form of heat to the surroundings. Luminescence centers with the largest quantum yields display the brightest emissions.

Lifetime is a measure of the transition probability, i.e. the average time that luminescence centers spend in the excited state prior to returning to the ground state. It is given as the time for the intensity to drop by $1 / \mathrm{e}$ from its original value. Lifetime is a characteristic and unique property of luminescence centers in a specific mineral structure, and it is highly improbable that two different emissions have the same decay time. The best way to combine the spectral and temporal nature of the luminescence emission is to obtain time-resolved spectra.

Time-resolved spectroscopy (Gaft and Panczer 2013) collects only the luminescence signal, by opening the detector gate a few ns or ms after the laser pulse. SuperCam has excellent synchronization between the laser pulse and the intensified CCD gate making it possible to explore luminescence with various decay periods. For this, one can use smart combinations of delays (the time when the detector gate opens with respect to the laser pulse) and open-gate duration. Lastly, Supercam is able to analyze the time decay of luminescence. A post-pulse detector gate with appropriate opening duration is set to various delay times (gate opening after laser pulse), and the integrated intensity of the signal is plotted versus the delay time. These data give further information on the nature of the electronic transition and, more practically, enable the identification of the emission center (Gaft et al. 2015).

Many lasers can induce luminescence when interacting with materials. Near-infrared lasers do not have enough energy to excite molecular luminescence. By contrast, ultra-violet lasers are very efficient and the emission is widely separated in energy from the Raman signal (as a reminder, Raman peaks are related to the excitation frequency; luminescence peaks are at a specific absolute frequency). The use of a UV laser is the option chosen by SHERLOC on Perseverance (Bhartia et al., this journal). However, to use the same laser oscillator for LIBS and TRR/L to minimize the resources, SuperCam is constrained to using a pulsed green beam at $532 \mathrm{~nm}$, which generates luminescence that spectrally overlaps the Raman signal.

It is important to stress that luminescence may be extremely effective in minerals (Gaft et al. 2020). Time-resolved luminescence using $532 \mathrm{~nm}$ stimulation can excite various emission centers in many minerals that emit in the wavelength range from $150 \mathrm{~cm}^{-1}(536.3 \mathrm{~nm})$ 
to $7060 \mathrm{~cm}^{-1}(852 \mathrm{~nm})$. These include $\mathrm{REE}^{3+}, \mathrm{Mn}^{2+}, \mathrm{Fe}^{3+}, \mathrm{Cr}^{3+}, \mathrm{Mn}^{3+}, \mathrm{V}^{2+}, \mathrm{Mn}^{4+}, \mathrm{Ti}^{3+}$, $\mathrm{Ag}^{+}, \mathrm{Bi}^{2+}, \mathrm{Pb}^{+}, \mathrm{Ni}^{2+}$, and $\mathrm{S}^{2-}$. For many of these emission centers, the detection is unambiguous but the quantification is challenging. In addition, many organic compounds exhibit a strong and short-lifetime (ns) luminescence signal and in some instances (e.g. pigments like chlorophyll), this luminescence is specific to the compound.

Fluorescence time scales are typically on the order of nanoseconds for organics while they can be much longer, from micro- to milliseconds, for most emission centers in minerals (Liu et al. 2017). Phosphorescence occurs via a similar mechanism, but it involves electronic transitions between states of differing spin. These "spin-forbidden" transitions, although spectrally similar to fluorescence, occur over significantly longer time scales (1 ms to $10 \mathrm{~s}$ for most organic molecules). The term "luminescence" encompasses both of these photoninduced mechanisms, referred to hereafter for SuperCam as Time-Resolved Luminescence (TRL).

\subsubsection{Visible and Near Infrared Spectroscopy (VISIR)}

Visible to near IR spectroscopy has been used from Mars orbit for nearly two decades. OMEGA on Mars Express (Bibring and Langevin 2008) and CRISM on Mars Reconnaissance Orbiter (Murchie et al. 2007) have made remarkable discoveries from orbit on the alteration mineralogy of the Martian surface. On the surface, Mastcam on Curiosity has several band filters in the 0.440-1.015 $\mu \mathrm{m}$ range (Malin et al. 2017). ChemCam covers the VIS range up to $850 \mathrm{~nm}$, which has allowed Johnson et al. $(2015,2017)$ to constrain the mineralogy of several iron-bearing minerals (e.g., hematite, olivine, and ferric sulfates). Near-IR investigation from the surface of Mars is new, especially in conjunction with LIBS to clear dust away. Both SuperCam onboard Perseverance and MarSCoDe onboard the Chinese Tianwen-1 rover carry similar VIS-IR channels with different fields of view. Whereas IR spectrometers from orbit are imagers, the two instruments on the ground are point spectrometers and will have a much smaller footprint, though on Mars 2020 it is complemented by Mastcam-Z's multispectral capabilities (Bell et al., this journal). Ground-based IR spectroscopy will for the first time give ground truth that can be compared to orbital IR data.

Passive VISIR spectroscopy, also known as VIS-NIR-SWIR reflectance spectroscopy, is widely used for the detection and identification of both organic and inorganic compounds. It relies on the targets being illuminated by artificial light sources in the laboratory or by the Sun for field work and planetary exploration. It exploits the fact that molecules absorb frequencies that are characteristic of their structure. Such absorption occurs at resonant frequencies when the frequency of the absorbed radiation matches the molecular vibrational frequencies (Fig. 5). Typical vibrational frequencies range from $\sim 10^{13}$ to $5.10^{14} \mathrm{~Hz}$ and more, covering at least the $0.6-30 \mu \mathrm{m}$ range. By convention, the $0.7-2.5 \mu \mathrm{m}$ range is called near-infrared range (NIR) and is the object of our study. For simplicity, we subdivide it between SuperCam's VIS range below $0.85 \mu \mathrm{m}$ and its IR range from 1.3 to $2.6 \mu \mathrm{m}$. Absorption bands in the reflectance spectra are usually sharper in IR than in the VIS range with the exception of some rare spin-forbidden transitions. Note that Mastcam has three broad RGB filters ( $\sim 40 \mathrm{~nm}$ width), and nine narrow filters per camera ( $\sim 10 \mathrm{~nm}$ width), which overlay SuperCam's VIS range and fill a gap between 850 and $1040 \mathrm{~nm}$. More details can be found in Bell et al. (this journal).

A major cause for IR absorption is a change in the dipole moment of a molecule as it vibrates or rotates. Absorptions in the IR are typical of molecular species that have a small difference between their rotational and vibrational states. Absorption bands in the NIR range are weak because they arise from vibrational overtones and combination bands (two molecular vibrations excited simultaneously). By comparison with longer IR wavelengths, however, 
Fig. 7 Orbital reflectance spectra from the Compact Reconnaissance Imaging Spectrometer for Mars (CRISM, Murchie et al. 2007) that are representative of the primary and secondary minerals found on Mars, restricted to the SuperCam VISIR wavelength range, calibrated to approximate surface reflectance, and ratioed to spectrally neutral terrains to suppress surface dust and residual atmospheric signatures (Viviano-Beck et al. 2014)

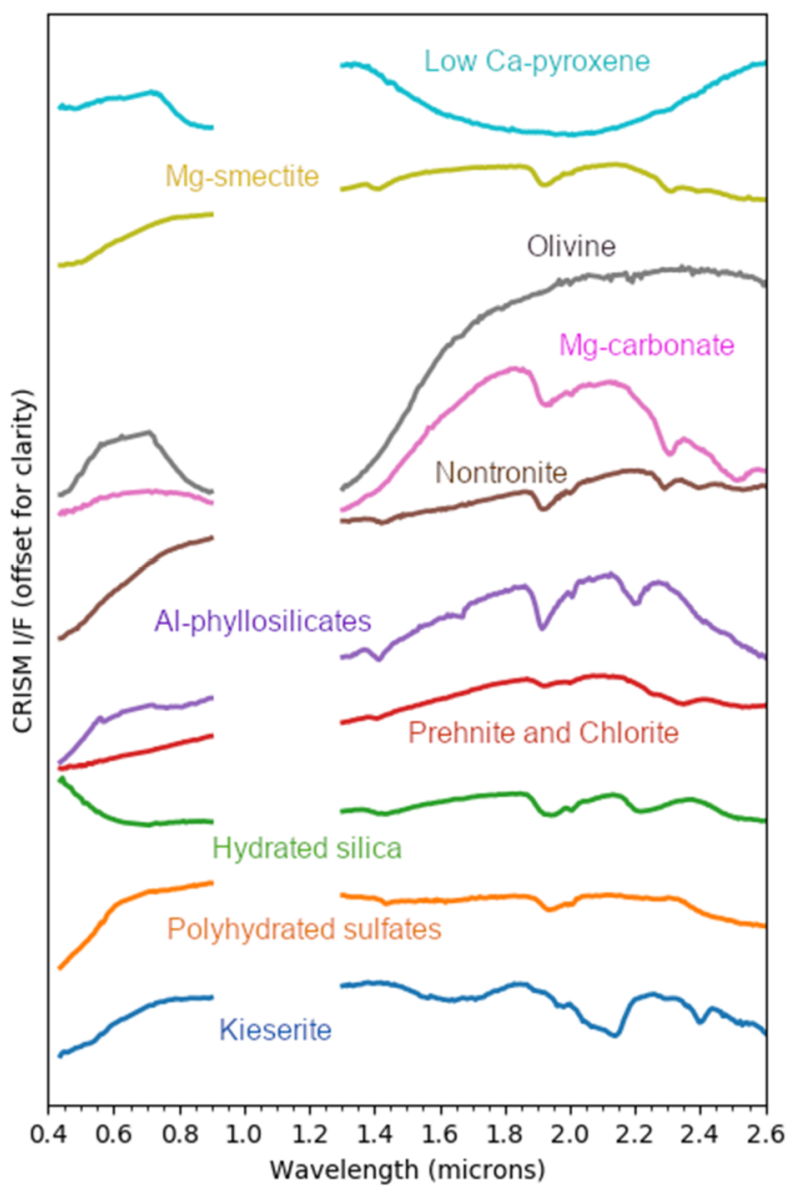

NIR bands have a high signal-to-noise ratio thanks to the solar emission spectrum being intense in this range, and to the fact that NIR wavelengths have deeper path lengths. The band depth of a compound in the NIR is typically correlated to concentration, optical absorption coefficient and grain size, though the greater penetration depth makes quantification using NIR spectroscopy challenging. IR spectroscopy using the Sun as a source is an effective way to identify various functional groups of interest for planetary science, especially on dust-free regions. This is where the coupling of IR spectroscopy with LIBS is advantageous.

The SuperCam VISIR wavelength range $(0.40-0.85 \mu \mathrm{m}, 1.3-2.6 \mu \mathrm{m})$, with the help of Mastcam-Z's narrow filters to cover the $800-1000 \mathrm{~nm}$ range, provides identification of many minerals (e.g. Murchie et al. 2009; Ehlmann and Edwards 2014) known or expected to be found in the geological record on Mars (Fig. 7):

$\checkmark$ Iron oxides and oxyhydroxides characterized by electronic transition and charge transfer absorptions extending from the ultraviolet to NIR and, less frequently, by narrow spinforbidden features of $\mathrm{Fe}^{3+}$ transitions observed in ChemCam passive spectra;

$\checkmark$ Ortho- and chain silicates through $\mathrm{Fe}^{2+}$ crystal field transitions that produce large absorption bands around 1.0 and $2.0 \mu \mathrm{m}$. The presence of the latter band discriminates pyroxene from olivine; its position strongly depends on the crystal chemistry of the pyroxene; 
$\checkmark$ Sheet silicates (clays, serpentine, talc, etc.), through the first harmonics of the fundamental vibrational mode of the hydroxyl radical $\mathrm{OH}(1.4 \mu \mathrm{m})$ and through its combination with the transverse vibrational modes of $\mathrm{Al}-\mathrm{OH}(\sim 2.2 \mu \mathrm{m})$ and $\mathrm{Fe}$ or $\mathrm{Mg}-\mathrm{OH}$ $(\sim 2.3 \mu \mathrm{m})$;

$\checkmark$ Sulfates (mono- and polyhydrated) through combinations and overtones of $\mathrm{OH}^{-}$or $\mathrm{H}_{2} \mathrm{O}$ bending and stretching fundamentals $(1.4 \mu \mathrm{m}, 1.9 \mu \mathrm{m})$ and $3 v_{3}\left(\mathrm{SO}_{4}\right)^{2-}$ overtone $(\sim 2.4 \mu \mathrm{m})$.

$\checkmark$ Carbonates through overtones and combinations of C-O stretching and bending vibrations $\left(3 v_{3}\right.$ at $\sim 2.3 \mu \mathrm{m}$ and $\left(v_{1}+2 v_{3}\right)$ at $\left.\sim 2.5 \mu \mathrm{m}\right)$. The wavelengths of their minima identify major cations and discriminate between different polymorphs. Mg-rich anhydrous carbonates exhibit minima at shorter wavelengths for the two bands in comparison with carbonates mostly containing $\mathrm{Ca}$ and $\mathrm{Fe}$.

$\checkmark$ Biologically-relevant compounds such as ammonium, borates, nitrates, and phosphates through overtones and combinations of $\mathrm{N}-\mathrm{H}, \mathrm{B}-\mathrm{O}, \mathrm{N}-\mathrm{O}$, and $\mathrm{P}-\mathrm{O}$ stretching and bending vibrations.

$\checkmark$ Molecular water (adsorbed, interlayer, water-ice) and $\mathrm{H}_{2} \mathrm{O}$-bearing salts in general through combinations and overtones of bending and stretching fundamentals (1.4-1.6 $\mu \mathrm{m}, 1.9-2.1 \mu \mathrm{m})$.

In addition, VISIR spectroscopy may be used to identify complex organic compounds from absorptions at $1.7 \mu \mathrm{m}, 2.15 \mu \mathrm{m}$, and 2.3-2.5 $\mu \mathrm{m}$ due to various combinations of $\mathrm{CH}_{2}$ and $\mathrm{CH}_{3}$ asymmetric and symmetric stretch, as well as $\mathrm{C}=\mathrm{C}$ and $\mathrm{CH}$. Finally, SuperCam detects atmospheric $\mathrm{CO}_{2}, \mathrm{CO}, \mathrm{H}_{2} \mathrm{O}$, and $\mathrm{O}_{2}$ (IR and 700-850 nm). The full spectral range is used to measure scattered light diagnostic of aerosol size distribution, composition, and opacity. These measurements will be made by fitting the observed sky radiance to multiple-scattering discrete-ordinate radiative-transfer models with gas absorption handled by the correlated-k method, as has been done with ChemCam (McConnochie et al. 2018).

\subsubsection{Complementarity of the Techniques}

Raman scattering and IR absorption are both vibrational spectroscopies, even if they are based on different physical processes (absorption versus inelastic scattering of light). IR absorption requires that a vibrational mode of the molecule has a change in dipole or charge distribution associated with it; only then can radiation of the same frequency interact with the molecule and raise it to an excited vibrational state. Raman scattering requires that a vibrational mode of the molecule leads to a change in polarizability. The intensity of the Raman scattering is proportional to the capability of the molecule to form instantaneous dipoles. Therefore, Raman and IR spectra may enable the identification of similar compounds but overall they provide complementary information.

Vibrations that have strong Raman intensities often have weak IR intensities and vice versa (Larkin 2011). The selection rules in quantum physics inform us whether such a molecule will be visible in IR (change in dipole moment) or in Raman (change in polarizability). As a classic example, the symmetric stretch of carbon dioxide is not IR active (no change in the net molecular dipole) and is Raman active (change of molecular polarizability). Conversely, the asymmetric stretch is IR active and not Raman active. The same is true for the bending motion. This does not occur with all molecules, but often times, the IR and Raman spectra provide complementary information about many of the vibrations of molecular species. Practically, in the case of SuperCam the choice of the technique to be preferred will depend on the performance of each technique. As a preview, after modeling the signal-to-noise ratio for each technique, we find that carbonates, phosphates, sulfates and 
framework silicates are best seen in Raman; sheet silicates and ices by IR; the chain silicates are divided: pyroxene by VISIR, and olivine by Raman (the latter in part due to missing the region from 1.0 to $1.3 \mu \mathrm{m}$ ). Metal oxides, which absorb too much of the incident light, are not clearly observed by either, except that the VIS spectral range is diagnostic of the iron oxidation state. The metal oxides can also be identified elementally by LIBS. The situation may change depending on grain sizes or illumination conditions.

Finally, LIBS is locally destructive for the target. Fau et al. (2019) studied the effect of LIBS laser shots on the Raman TRR signature of the target. They showed that the structure of samples with low optical absorption coefficients at the LIBS wavelength is preserved. By contrast, minerals with high optical absorption coefficients at the LIBS wavelength can be severely affected by LIBS laser shots with local amorphization, melting and/or phase transformation. Anyway, because the analytical footprint of TRR is much larger than the LIBS laser spot, the possible alteration of Raman signatures due to LIBS should not be detected in the TRR analyzed by SuperCam, even in the case of highly absorbing minerals (Fau et al. 2019). Dust is detrimental for Raman analysis as it absorbs the incident laser light and contributes a strong background in the TRR spectra thereby preventing TRR/L analysis of the underlying target. A strategy to use LIBS first, which has the advantage to remove dust, and then TRR/L, or the reverse will have to be decided during mission operations to maximize the science return.

\section{Instrument Implementation}

SuperCam's techniques benefit from the ability to operate at remote distances. The distance capability is a key driver of the design, with a distance range capability specific to each technique. From this, we can establish performance requirements that lead to measurement and functional requirements with a higher level of detail. This is the classical traceability matrix approach. The design of SuperCam is inseparable from accommodation constraints of the rover on which it is mounted, especially when it comes to the accuracy of pointing at remote targets. For this reason, we describe the pointing requirements that are prescribed by SuperCam to the rover project. A description of SuperCam's architecture concludes this part.

\subsection{Distance Capability}

SuperCam's techniques all benefit from the ability to operate at remote distances on raw targets without prior sample preparation. From a scientific point of view, the broader the distance range, the better, but in practice, accommodation constraints also drive the distance limits. Figure 8 summarizes SuperCam's remote sensing capabilities.

To benefit from a $\pm 180^{\circ}$ azimuth and $\pm 90^{\circ}$ elevation pointing capability, the SuperCam telescope is mounted at the top of the rover mast. This sets the minimal distance to the first possible targets on the ground at approximately $2.0 \mathrm{~m}$, which is the vertical distance from the outer face of the telescope's Schmidt plate to the ground at the front of the rover. Therefore, we chose $2 \mathrm{~m}$ as a minimum distance for Mars targets. The calibration targets at the rear of the rover deck are one exception since they are located at an average distance of $1.56 \mathrm{~m}$ (Manrique et al., this journal).

RMI and VISIR spectroscopy could operate up to infinity, but in practice they are limited by the atmospheric transparency or the horizon. The long distances for active experiments are limited by the optical performance of the telescope, whose size is bounded by the volume available at the top of the rover mast. Considering this constraint and the need to reach 


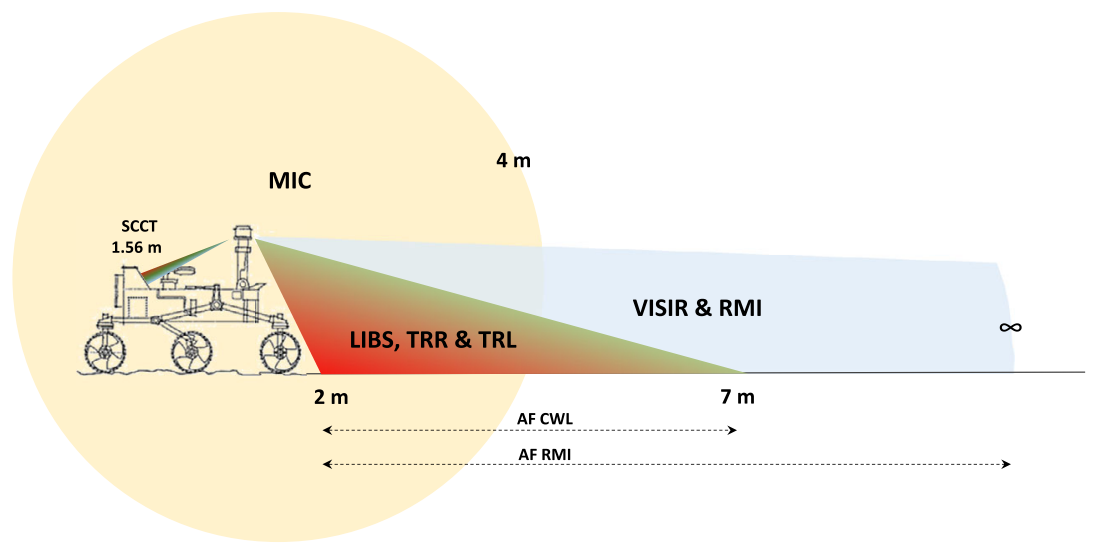

Fig. 8 SuperCam remote sensing capability. All distances are from the center of the Mast-Unit external window. With its laser beams, LIBS, time-resolved Raman (TRR) and Luminescence (TRL) spectra can be obtained from $2 \mathrm{~m}$ (the closest distance to the ground) up to $7 \mathrm{~m}$ away. Imaging (RMI) and passive spectroscopy (VISIR) have in principle no limit in distance. Each technique can probe the calibration targets (SCCT) at $\sim 1.56 \mathrm{~m}$. There are two autofocus methods (AF): the image-based autofocus (RMI-AF) can be used at any distance, while the laser-based autofocus (CWL-AF) is limited to distances of less than $7 \mathrm{~m}$

the required signal-to-noise ratio for each technique (see Sect. 3.2), $7 \mathrm{~m}$ was chosen as the maximum required distance for LIBS and TRR/L. This corresponds to an area more than $100 \mathrm{~m}^{2}$ that can be covered by SuperCam from a given rover location. This area contains the arm work zone (approximately $2.6 \mathrm{~m}$ in the front of the rover), which is a priority because this is where the samples are collected from. Measurements at this distance benefit from high-resolution Navcam images, which aid in pointing at sub-centimeter targets of interest and to benefit from an accurate Navcam-based estimate ("seed") of their distance to SuperCam. For reference, $\sim 95 \%$ of ChemCam observations are performed closer than $4.5 \mathrm{~m}$, but certain targets (iron meteorites) were shot at up to $9.5 \mathrm{~m}$. With its improved telescope, SuperCam is expected to cover a broader area around the rover compared to ChemCam.

SuperCam has two autofocus (AF) methods: one image-based using RMI and the other one based on a $\mathrm{CW}$ diode laser. The AF methods both work well over the 1.56-7 $\mathrm{m}$ range for all techniques. The image-based autofocus works to infinity and will be used to support longdistance observations. Both methods now exist on ChemCam with similar performance.

The microphone records local pressure variations that are generated at a distance. However, because of its main constituent, the $\mathrm{CO}_{2}$ molecule, the Mars atmosphere absorbs sounds efficiently: at $4 \mathrm{~m}$ it adsorbs between $-0.8 \mathrm{~dB}$ and $-1.2 \mathrm{~dB}$ at $1 \mathrm{kHz}$ at $-20{ }^{\circ} \mathrm{C}$ (Williams 2001; Bass and Chambers 2001), compared to only $-0.01 \mathrm{~dB}$ on Earth at $20^{\circ} \mathrm{C}$. Furthermore, the high characteristic acoustic impedance of the Mars atmosphere weakens the coupling efficiency of an acoustic source by $20 \mathrm{~dB}$ compared to the same source on Earth. For this reason, a distance of $4 \mathrm{~m}$ was set as a maximum distance to record the LIBS shock wave with the microphone. This distance is also sufficient to collect all artificial sounds from the rover.

\subsection{Performance Requirements}

This section describes the overarching requirements that encompass the science intent described above, as well as distance requirements. They are called Level-4 requirements by the 
Table 1 Relationship between SuperCam science objectives and the mission goals. Dark/light grey highlight the major/minor contributions from SuperCam

\begin{tabular}{|c|c|c|c|c|c|c|c|c|}
\hline Mars 2020 & 苍 & 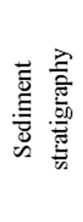 & 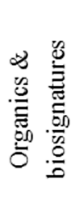 & 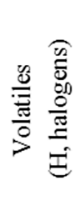 & 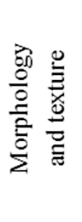 & 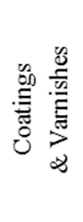 & 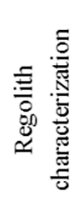 & 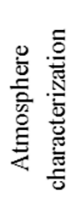 \\
\hline A. Geologic diversity & & & & & & & & \\
\hline B1. Habitability & & & & & & & & \\
\hline B2. Bio-signatures & & & & & & & & \\
\hline B3. Past life & & & & & & & & \\
\hline C. Cache samples & & & & & & & & \\
\hline D2. Dust & & & & & & & & \\
\hline D3. Weather & & & & & & & & \\
\hline
\end{tabular}

rover project. When necessary, quantitative tests are identified to verify requirements either using the Mast Unit alone or, more often, the whole instrument (Wiens et al., this journal).

Table 2 presents the performance requirements for elemental chemistry, for atmospheric constituents, and for mineral detection. It is worth pointing out that the connection between the eight goals (Table 1) and these top-level performance requirements (Table 2) is multifaceted, considering the performance needed to attain the goals, but also paying attention to recently demonstrated capabilities (e.g., with ChemCam for elemental compositions) and attainable instrument performance.

In terms of elemental-composition capabilities, major-element accuracies of $\pm 10 \%$ are generally sufficient to distinguish broad classes of rocks. An example is the total alkalisilica (TAS) diagram, used for igneous rocks, where this level of accuracy distinguishes basalts from andesites from dacites and rhyolites in silica, and trachy-basalts and trachyandesites from the lower-alkali counterparts. The ability to trace liquid lines of descent is perhaps more important, as it can link evolved igneous rocks to their parental magmas (e.g., Sautter et al. 2016). In reality, much of SuperCam's exploration will likely be in sedimentary terranes, where distinguishing different provenances of the sediments is useful. Based on ChemCam experience, a 10\% accuracy among all major elements and 20\% among the listed trace elements allows very useful delineation (e.g., Edwards et al. 2017; Bedford et al. 2019).

The atmospheric requirements are based on experience with ChemCam measurements and proven capabilities (McConnochie et al. 2018). These requirements are generally sufficient to observe seasonal variations, and to compare one Mars year to the next. While SuperCam is not primarily an atmospheric observatory, it will nicely complement the work carried out by MEDA and will continue the type of ground-based observations of atmospheric water, dust, carbon compounds, and oxygen that ChemCam has done since early 2013.

Mineral requirements were based much more on expected capabilities, given the lower level of experience in this area and the fact that the abundances of minerals are difficult to quantify with VISIR and TRR/L, while their identification, and often some details on relative abundances, are quite realistic. The table was based on capabilities of orbital instruments in the VISIR spectral range (Viviano-Beck et al. 2014) and the knowledge that they have yielded on the types of minerals and science questions relevant for habitable environments on Mars. It was also based on our experience so far with remote Raman spectroscopy. 

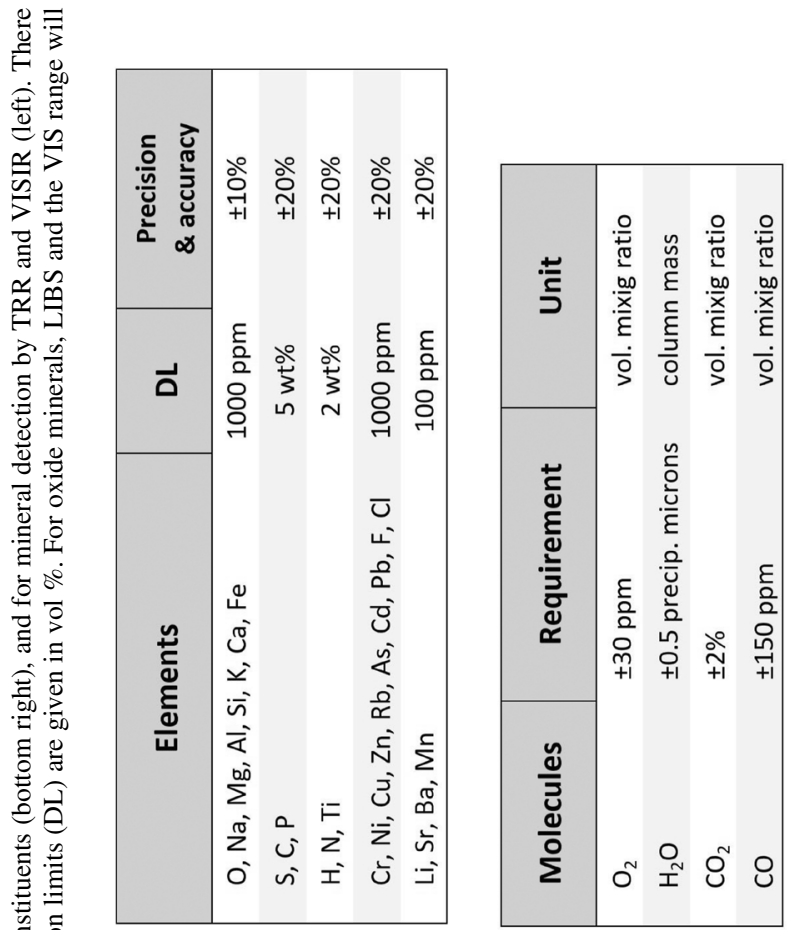

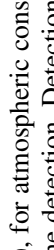

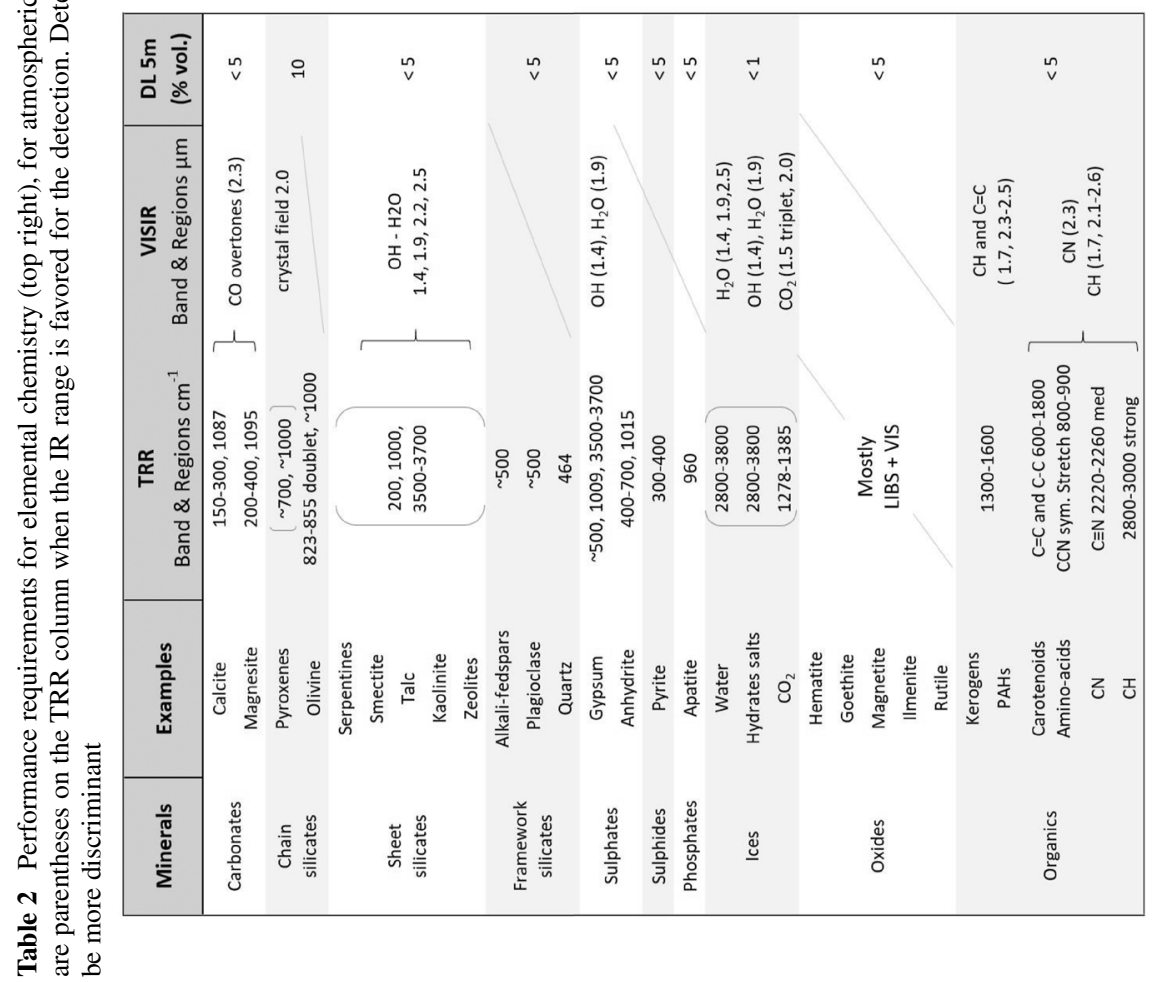

N

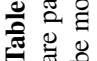


Volume $\%$ for the detection limits (DL) are only indicative, and are used for test purposes (Wiens et al. this journal). It is clear that there is not a direct, linear or not, relationship between the amount of a given mineral and the spectral signature strength. Later, the performance requirements are translated into signal-to-noise ratios, which was used by SuperCam engineers for the instrument design. In the end, the mineral specifications are viewed more as goals, but as described in this manuscript and Wiens et al. (this journal), they are realistic ones.

\section{Hence:}

- The instrument shall be able to measure elemental composition on the surface of Mars for elements given in Table 2 with the specified detection limit, precision and accuracy, from 2 to $7 \mathrm{~m}$.

This requirement sets the objectives for LIBS. These are very similar to ChemCam (Maurice et al. 2012; Wiens et al. 2012) for both the list of elements to be detected, the detection limit, precision, and accuracy given at $5 \mathrm{~m}$. Based on ChemCam's experience, we know that if the potassium line at $767 \mathrm{~nm}$ can be detected with an SNR > 64 at $5 \mathrm{~m}$, then the laser coupled well with the target, and the signal to noise was sufficient for the all the objectives of Table 2 (top right) to be achieved.

- The instrument shall be able to measure depth profiles of elemental composition on the surface of Mars, for elements given in Table 2 (top right), from 2 to $7 \mathrm{~m}$, and at the location of the calibration targets.

This is related to LIBS again and its ability to probe the target surface as a function of depth, and to explicitly investigate coatings or varnishes. The validation test is to penetrate $0.5 \mathrm{~mm}$ in a rock of hardness 5 (Mohs scale) at $5 \mathrm{~m}$.

- The instrument shall be able to determine the atmospheric concentrations of molecular species given in Table 2 (bottom right), with specified detection limits.

Based on ChemCam's experience, this requirement is automatically validated when the more general specification for LIBS elemental composition is met. VISIR contributes to these detections.

- The instrument shall be able to detect mineralogical composition on the surface of Mars as given in Table 2 (left), with the specified detection limit, from $2 \mathrm{~m}$ to $7 \mathrm{~m}$, at the location of the calibration targets and up to the horizon whenever possible.

This requirement sets the objectives for VISIR and TRR together, and it suggests which technique is most appropriate. At the time of the SuperCam proposal, little was known about Raman spectroscopic capabilities at long distance, so this requirement has been reformulated during the course of the project. For Raman, the validation test is to detect carbonate with SNR $>20$ at $7 \mathrm{~m}$. For VIS spectroscopy, the requirement is to reach SNR > 50 at $800 \mathrm{~nm}$, and for IR spectroscopy, SNR > 56 at $2.6 \mu \mathrm{m}$ for carbonates of reflectance 0.5 . Both VIS and IR can go to infinity and their signal-to-noise ratio is independent of the distance.

- The instrument shall be able to acquire color context images of the areas investigated for elemental and mineralogical composition and organics, from $2 \mathrm{~m}$ to infinity, and at the calibration target location.

While the ChemCam RMI was monochrome, the one on SuperCam is designed to acquire color images. Everything else, including resolution and field of view, is the same. RMI images must have the capability to document the distribution of elements, mineral classes, organics, and/or morphologies at the sub-millimeter scale. Hence its field of view needs to overlap those of the other techniques.

- The instrument shall be able to record audio signals from $100 \mathrm{~Hz}$ to $10 \mathrm{kHz}$ on the surface of Mars, with a SNR $>3$ for LIBS impacts at $4 \mathrm{~m}$. 
- The investigation will carry its own set of calibration targets for each technique (Manrique et al., this journal): at least 20 calibration targets for LIBS that should be representative of major rock units; a Ti target for spectral calibration by LIBS; two targets for TRR; two targets, white $>95 \%$ reflectance and dark $<5 \%$ reflectance, for VISIR radiometry with magnets to minimize dust over $\geq 1 \mathrm{mrad}$; a geometric target to measure resolution and modulation transfer function (MTF) of the imaging capability, and three red-green-blue (RGB) targets with magnets for white balance.

\subsection{Measurement and Functional Requirements}

The previous sections have allowed us to identify performance requirements for the five different analytical techniques. From there we derive below, for each of them, the measurement and functional requirements that are called Level-5 requirements. We are separating VIS and IR ranges for clarity.

- For LIBS, the infrared laser needs to be focused to obtain an irradiance $>10 \mathrm{MW} / \mathrm{mm}^{2}$ at remote distances from 1.5 to $7 \mathrm{~m}$. This irradiance is calculated within a closed contour that contains over $75 \%$ of the energy deposited on the target. The energy on target should be above $12 \mathrm{~mJ}$ over the Mast Unit $\left[-30^{\circ} \mathrm{C},+10^{\circ} \mathrm{C}\right]$ temperature range, and above $15 \mathrm{~mJ}$ at one temperature within that temperature range.

Plasma light needs to be collected and analyzed between $245 \mathrm{~nm}$ and $853 \mathrm{~nm}$, except for two wavelength gaps between 340-385 nm and 465-536 nm imposed by the optical design (details in Sect. 4.4.4). The FWHM resolution across the whole wavelength range shall be better than $0.2 \mathrm{~nm}$ for wavelengths below $500 \mathrm{~nm}$ and better than $0.65 \mathrm{~nm}$ for wavelengths above $500 \mathrm{~nm}$.

For the SuperCam laser energy and size of its telescope, the diameter of the ablation is about $500 \mu \mathrm{m}$ and the hot part of the plasma, characterized by the atomic-emissionline emission, is approximately of the same size (Sallé et al. 2006). The field of view of the LIBS investigation must capture this plasma; the LIBS light collection shall be $\sim 0.8 \mathrm{mrad}$.

- For TRR, the first requirement is to induce an irradiance between 10 and $30 \mathrm{~kW} / \mathrm{mm}^{2}$ per pulse with the green laser, at distances from 1.5 to $7 \mathrm{~m}$. The energy should be above $9 \mathrm{~mJ}$ over the $\left[-30{ }^{\circ} \mathrm{C},+10^{\circ} \mathrm{C}\right]$ temperature range, and above $11 \mathrm{~mJ}$ at one given temperature over the same temperature range.

Raman and luminescence photons need to be detected between $150 \mathrm{~cm}^{-1}(536 \mathrm{~nm})$ and at least $4400 \mathrm{~cm}^{-1}(695 \mathrm{~nm})$, while the laser elastically scattered light is rejected. A goal is to reach $7000 \mathrm{~cm}^{-1}$ for TRL. The resolution across the whole wavelength range shall be better than $12 \mathrm{~cm}^{-1}$.

The minimum signal integration time shall be $\leq 100 \mathrm{~ns}$, and can be increased up to $60 \mu \mathrm{s}$. The delay between the laser firing and the start of the Raman exposure window shall be adjustable between 0 and $500 \mu$ s (for luminescence).

The field of view for TRR/L shall match the LIBS FOV to investigate the same area. Thus, it shall be $\sim 0.8 \mathrm{mrad}$.

- For VIS, the wavelength coverage shall be from 400 to $853 \mathrm{~nm}$ except for a gap between 465 and $537 \mathrm{~nm}$, with a resolution across the whole wavelength range better than $0.65 \mathrm{~nm}$. There is no limit to the distance to target.

The field of view of the passive VIS shall match the LIBS FOV to investigate the same area. Thus, it shall be $\sim 0.8 \mathrm{mrad}$.

- For IR, the wavelength coverage shall be from 1.3 to $2.6 \mu \mathrm{m}$. The signal-to-noise ratio over this range shall be $>57$ for an $80 \mathrm{~s}$ integration time (including dark measurements) and 
an irradiance of $300 \mathrm{~W} / \mathrm{m}^{2}$ on target and an albedo of 0.3 over the Mast Unit $\left[-40{ }^{\circ} \mathrm{C}\right.$; $-5{ }^{\circ} \mathrm{C}$ ] temperature range. The resolution across the whole wavelength range shall be better than 32 wavenumbers $\left(\mathrm{cm}^{-1}\right)$ : over the wavelength range, the resolution shall be better than $5.4 \mathrm{~nm}$ at $1.3 \mu \mathrm{m}$ and better than $21.5 \mathrm{~nm}$ at $2.6 \mu \mathrm{m}$. At least 256 wavelength steps shall be implemented to allow oversampling of the IR range. There is no limit to the distance to target.

The relative response between two adjacent (wavelength) bins shall be known with a precision better than $1 \%$. The infrared Spectrometer (IRS) absolute spectral response shall be known with a precision better than $20 \%$.

The field of view of the passive IR shall match the LIBS and TRR/L FOVs to investigate the same area. More flexibility is accepted here to collect enough light, as long as the FOV stays smaller than the area that is cleared of dust by the LIBS. The FOV requirement is set to be $\sim 1.2 \mathrm{mrad}$.

- For RMI, a color (using per-pixel RGB filters) image of any SuperCam targetable area around the rover needs to be acquired. There is no limit to the distance to target. The RMI must support in priority the characterization of the areas closed to the targets analyzed by LIBS, TRR, and VISIR. As such, it will operate at the calibration target distance, $1.56 \mathrm{~m}$, and from 2 m up to infinity.

The RMI shall have a spatial resolution better than $80 \mu \mathrm{rad}$, and an MTF above 0.20 at 20 line pairs $/ \mathrm{mm}$ over a field of view higher than $10 \mathrm{mrad}$, when in good focus on a target at a distance beyond $2 \mathrm{~m}$. The signal-to-noise ratio of RMI images shall be larger than 200 (after binning) at $50 \%$ of the dynamics for irradiance of $300 \mathrm{~W} / \mathrm{m}^{2}$ (on target) and albedo of 0.4 . The RMI should include an auto-exposure capability, around a given nominal exposure time.

Relative radiometric calibration of the RMI between RGB pixels shall be performed with an accuracy of $\pm 20 \%$. The flat field shall be known with a pixel-to-pixel accuracy of $\pm 5 \%$.

The RMI field of view shall be $>15$ mrad to cover the range of grain sizes (Fig. 2) and to capture the context of the analytical techniques.

- For MIC, acoustic data from $100 \mathrm{~Hz}$ to $10 \mathrm{kHz}$ need to be acquired to record the laserinduced sparks generated at $4 \mathrm{~m}$ with a signal-to-noise ratio $>3$. Amplification gains shall be implemented if necessary. Two sampling frequencies are implemented at $25 \mathrm{kHz}$ and $100 \mathrm{kHz}$.

For combined LIBS and microphone studies, synchronization between the microphone and the infrared laser is required. For the standalone mode, to support atmospheric studies or to record rover noises, the max recording time shall be $\geq 167 \mathrm{~s}$ at $25 \mathrm{kHz}$ sampling. The microphone shall not be saturated under $1 \sigma$ of the wind conditions specified in the environmental requirement documents of the project.

Along with these requirements, there are many technical specifications, the most relevant being:

- Optical axes for LIBS, TRR, and VISIR shall be co-aligned to within $0.35 \mathrm{mrad}$. The auto-focus (AF) capability shall be co-aligned with the same accuracy. All optical axes should be within $2 \mathrm{mrad}$ of the center of the RMI.

- SuperCam will implement two independent modes to focus autonomously the telescope, the first one using a dedicated Continuous Wavelength Laser (CWL-AF), and the second one relying on the imaging capability of the instrument (RMI-AF). The former one operates from 1.5 to $7 \mathrm{~m}$, and the latter one to any distance up to infinity. The requirement is to reach an accuracy at $\pm 0.4 \%$ of the exact distance to target in less than 2 min, starting from a seed distance at $\pm 5 \%$. 


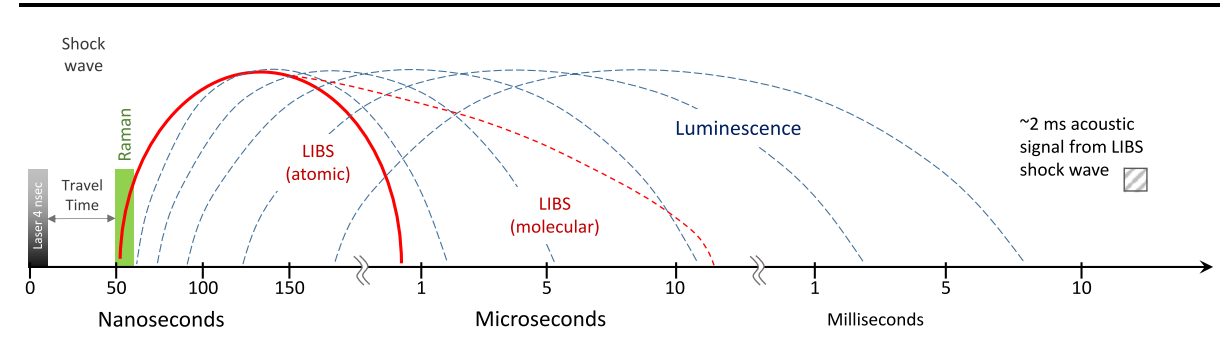

Fig. 9 Schematic chronogram of SuperCam techniques (TRR/L, LIBS, MIC) as seen from the Mast Unit. The laser duration is $4 \mathrm{~ns}$. Travel time ( 2 ways) is for targets at $\sim 7 \mathrm{~m}$. Raman scattering emission occurs during the laser pulse. LIBS starts when the laser beam vaporizes the target and lasts a few microseconds for atomic lines and longer for molecular lines. The luminescence can start as soon as the laser beam hits the ground, or later, and it lasts longer, up to milliseconds, depending on the material. The shock wave is produced as soon as the laser hits the target. Then the sound propagates to the instrument at $\sim 230 \mathrm{~m} / \mathrm{s}$

- SuperCam shall withstand being pointed at the Sun indefinitely in a sun safe configuration. SuperCam shall notify the Flight System before placing itself into a non-sun safe configuration. Conversely, the Flight System shall not allow SuperCam's boresight to point within $0.7^{\circ}$ ( + margins) of the Sun whenever SuperCam is in a non-sun safe configuration.

- The instrument critical functions shall be re-programmable in flight (software) or reparametrizable through parameters tables.

There is one difficulty inherent to the laser-induced investigations implemented by SuperCam that needs to be pointed out: the intrinsic time scales of LIBS, Raman, and luminescence are different by a few orders of magnitude, and the instrument must accommodate all of these (Fig. 9). The Raman signal has the shortest time scale as it lasts only the duration of the laser pulse, $\sim 4 \mathrm{~ns}$. The spectrometers shall frame this emission, as close as possible, also considering light travel time. The larger the time window on the CCD (gate), the more background is integrated, affecting the signal. The LIBS signal for atoms lasts much longer, up to a few microseconds. The spectrometers shall frame the LIBS emission, but the exposure duration can be relatively long (e.g. milliseconds on ChemCam) since the LIBS signal is so bright compared to the background. With a long exposure, there is also the opportunity to capture molecular lines, which can be spotted easily in the spectra. The luminescence signal starts during the laser pulse and extends from nanoseconds to milliseconds. For timeresolved luminescence, the spectrometers will have to implement a delay relative to the laser emission, and possibly a very large integration window.

\subsection{Pointing Requirements}

The capability of the rover to point at different targets with precision and accuracy is a huge contributor to the success of the investigation. In general, SuperCam requirements are the same as, or more stringent than ChemCam's.

- The size of SuperCam's targets of interest is smaller than $1 \mathrm{mrad}$. Therefore, the science team has requested a \pm 1 mrad in elevation (EL) and azimuth (AZ) accuracy for pointing the rover mast on Martian surface targets, which is more stringent than on MSL. In the open-loop mode (no feedback on actual pointing), the pointing accuracy in EL and AZ relative to the Rover Mechanical Frame is $\pm 9.5(2 \sigma)$ mrad per axis, which is not sufficient for SuperCam. To improve the accuracy, "way-points" can be used: stationary positions 
to always approach the target in the same EL and AZ directions. Based on the lessons learned from Curiosity, the open-loop accuracy with two way-points can approach $2 \mathrm{mrad}$ but sometimes with significant outliers. To do better, a feedback control based on the use of RMI images has been implemented (closed-loop correction) to reach $\pm 1 \mathrm{mrad}$. If Navcam full resolution images are used with closed-loop pointing, $\pm 2 \mathrm{mrad}$ accuracy can be reached without using way-points.

- For small rasters, a minimum controllable step size in EL and AZ for a pointing accuracy of $0.2 \pm 0.05(2 \sigma)$ mrad is implemented, as requested.

- LIBS depth profiles and RMI image quality are drivers for pointing stability. It shall be better than $80 \mu \mathrm{rad}$ over $2 \mathrm{~s}$ (RMI) and $100 \mu \mathrm{rad}$ over $10 \mathrm{~s}$ (LIBS), better than MSL (10 $\mu \mathrm{rad}$ over $20 \mathrm{~ms})$.

- Repeatability (precision) is understood to pertain when the rover is stationary. The goal is to reach better than $\pm 1 \mathrm{mrad}$, similar to what was measured on MSL. The requirement for the Mast design for repeatability in joint coordinates pointing accuracy is $\pm 2(2 \sigma) \mathrm{mrad}$, assuming no thermo-mechanical deformations.

- The rover will have the capability of carrying out SuperCam nighttime observations, including those requiring mast motions, when components are within their allowable flight temperatures.

- For the autofocus capability for each SuperCam target, the flight system will provide an estimate of the true distance to the target with an accuracy of $\pm 1 \%$ up to $7 \mathrm{~m}, \pm 5 \%$ from $7 \mathrm{~m}$ to $12 \mathrm{~m}$, and $\pm 10 \%$ from 12 to $200 \mathrm{~m}$, to be used as a seed distance.

All requirements (pointing, nighttime observations, AF capability) will be verified while on Mars.

\subsection{Architecture Overview}

As in the case of ChemCam, SuperCam is made of three parts (Fig. 10): the Body Unit, the Mast Unit, and the Calibration Targets (SCCT) that are somewhat independent of each other and were developed to a large extent in the United States, France, and Spain respectively.

The Mast Unit is mounted on the Remote Sensing Mast (RSM) camera plate, which also carries the Navcams and Mastcams (Fig. 11). A cover, also called the Remote Warm Electronic Box (RWEB) encloses the camera plate and Mast Unit. There are two openings in the RWEB: a small one for the microphone, a larger one for the telescope, which, with its window like an eye, gives the rover a distinctive cyclops look. The Mast Unit is mounted in such a way that its field of view is within the Navcam and Mastcam-Z fields of view at $1 \mathrm{~m}$ distance. The RSM can rotate $\pm 181^{\circ}$ in azimuth (AZ) and $\pm 91^{\circ}$ in elevation (EL).

The Body Unit is mounted on the Rover Accessory Mounting Panel (RAMP), which is the rover deck. Except for harnessing and the RAMP, the Body Unit is not in thermal contact with any other parts of the rover. More details can be found in Wiens et al. (this journal).

SuperCam calibration targets are mounted at the rear of the rover at a distance of $1.56 \mathrm{~m}$, the same range as for ChemCam for comparison of calibration data. To avoid laser reflection toward the Mast Unit, the calibration target holder is mounted on the rover such that the angle between its surface normal and the SuperCam boresight varies from 11 to 14 degrees, depending on which individual target is considered.. More details can be found in Manrique et al. (this journal). The smaller structure that holds just the alumina plates and the meteorite is tilted further upward to give a final angle of $\sim 6^{\circ}$ from normal to the instrument boresight.

The primary purpose that drove ChemCam's architecture in the first place was to simplify the Mast-Unit and Body-Unit interfaces with the rover and between each other. The same strategy is used on SuperCam (Fig. 12). Each unit has its own mechanical and thermal 


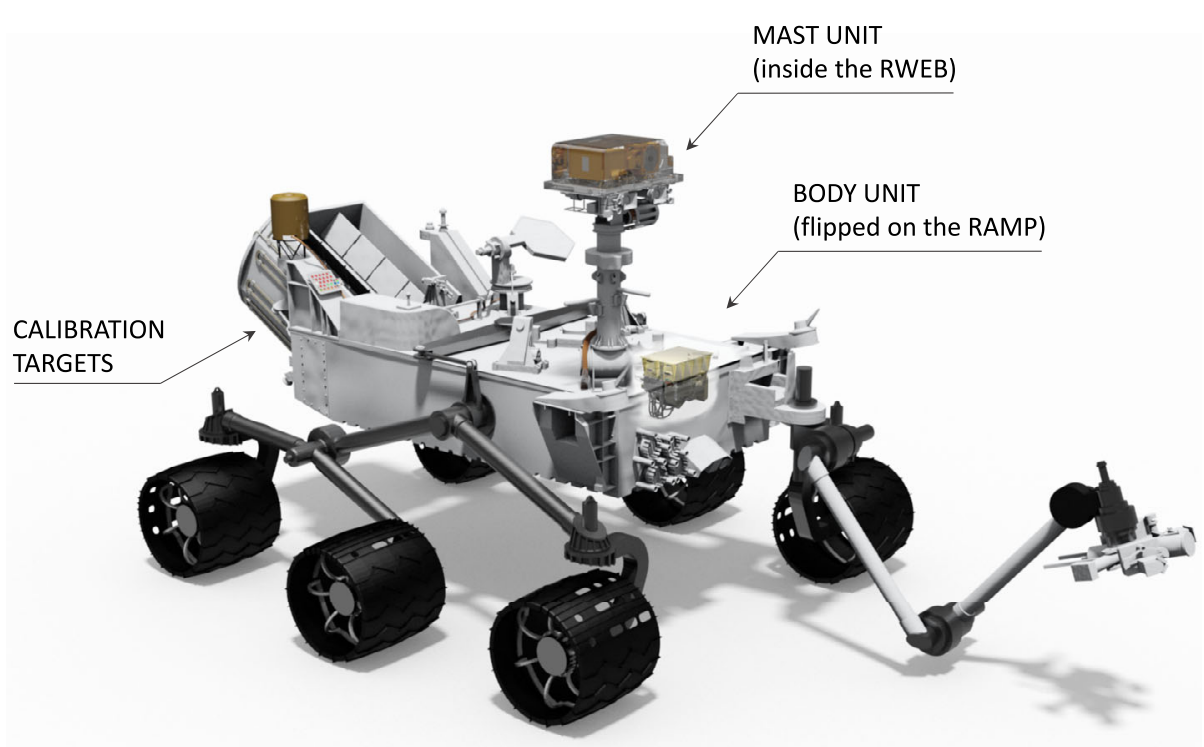

Fig. 10 Overview of SuperCam accommodation on the rover. The Mast Unit is encapsulated in the Remote Warm Electronic Box (RWEB). The Body Unit is mounted on the Rover Avionics Mounting Panel (RAMP)

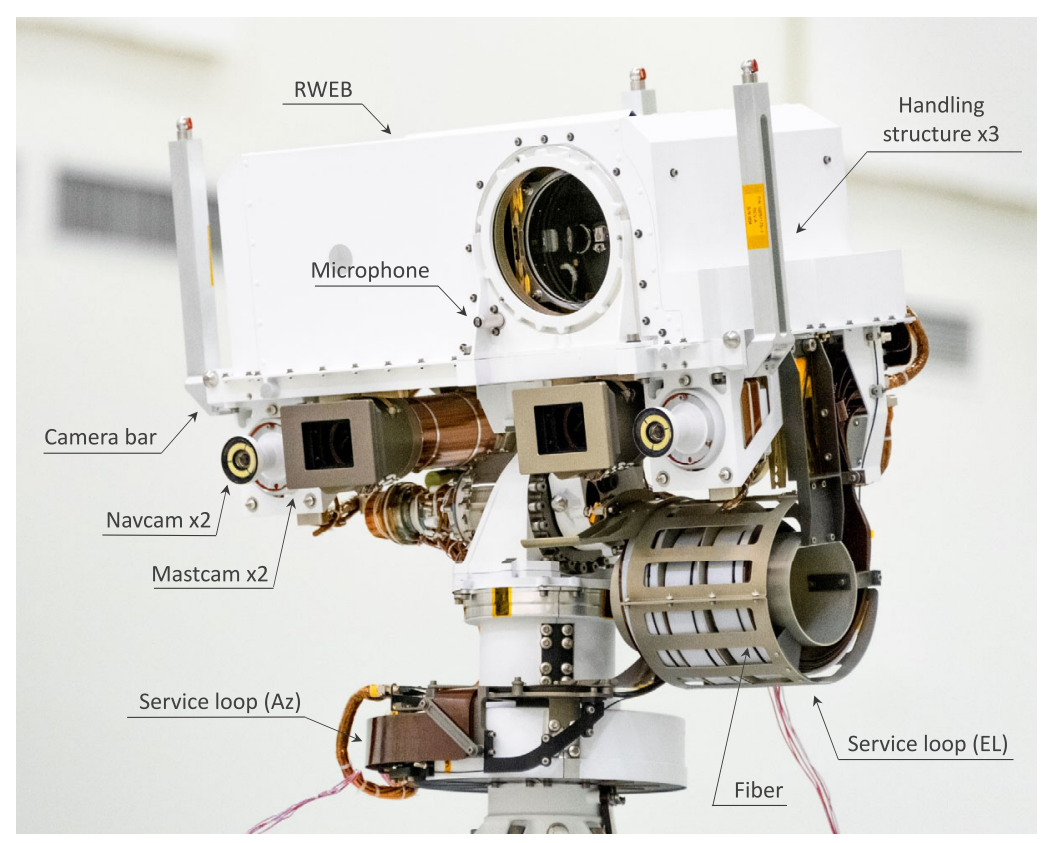

Fig. 11 The Mast Unit mounted on the camera bar inside the RWEB. Attached below the pairs of Mastcam and Navcam cameras. The optical fiber to the Body Unit is rolled into service loops for elevation (EL) and azimuth (AZ) motions. Three handling posts will be removed before launch. (Credit: NASA/JPL-Caltech) 


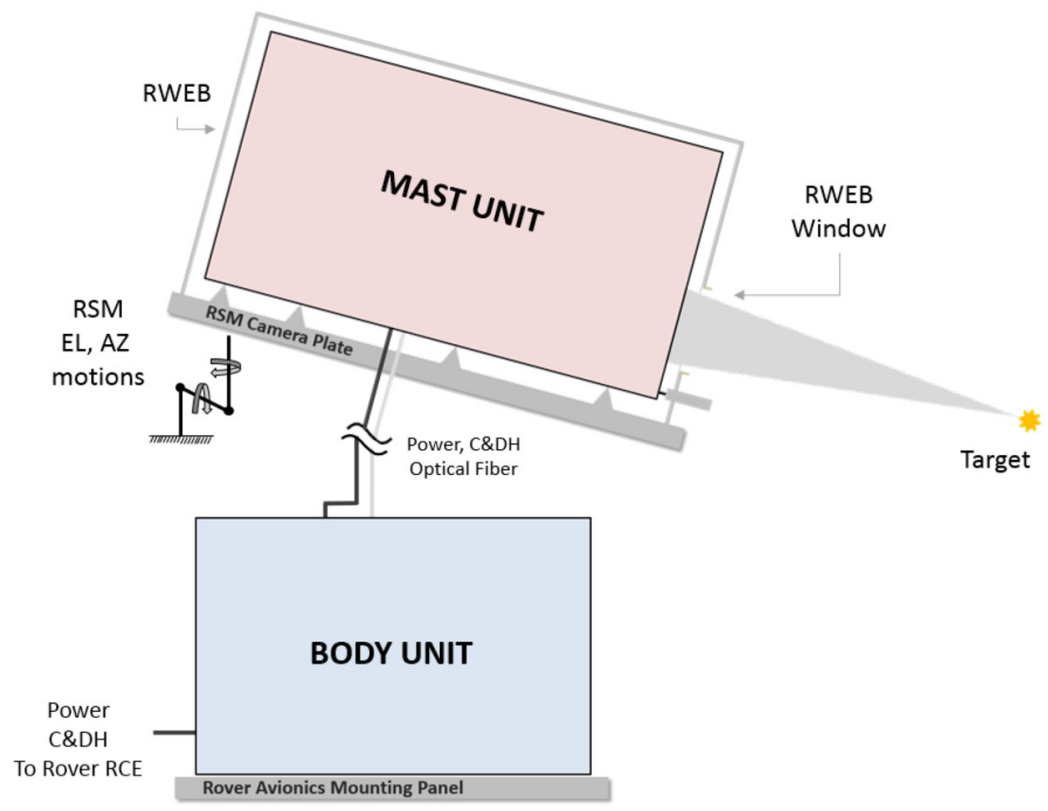

Fig. 12 SuperCam system overview and key interfaces

interfaces with the RSM or the RAMP. The telescope hole through the RWEB is the optical interface to the outside. It is closed by an optically flat window tilted at $3.5^{\circ} \pm 1.0^{\circ}$ to prevent laser reflections going backward to the telescope. The JPL provided fiber is the optical interface between the Mast Unit and the Body Unit. Service loops allow elevation and azimuth motions of the RSM while minimizing twisting the fiber. The fiber is $\sim 6 \mathrm{~m}$ long with no intermediate connector (Wiens et al., this journal).

Electrical and optical interfaces are summarized in Fig. 13. The Mast Unit has minimal connections to the rover, which reads temperature sensors (PRTs) and drives survival heaters. The Mast Unit power is provided only through the Body Unit. The Body Unit exchanges Command and Data Handling $(\mathrm{C} \& \mathrm{DH})$ telemetry with the rover computer (A or B side) and with the Mast Unit (Wiens et al., this journal). The JPL-provided harness connecting the 2 parts of the instrument has several intermediate connectors.

The microphone is mounted outside of the RWEB, on a $3 \mathrm{~cm}$ boom to separate the main signal from its echo off of the RWEB, and is pointed within $\pm 5^{\circ}$ of the laser beam axis. The microphone has a $180^{\circ}$ listening angle, unobstructed by other RSM structural elements.

Based on this architecture, RMI, MIC, and IR spectroscopy are exclusively located within the Mast Unit. The red/green lasers are in the Mast Unit, while the spectroscopic analysis for LIBS, TRR, and in the VIS range occur in the Body Unit.

\section{Mast-Unit Design}

This part describes how the Mast Unit was built in accordance with the requirements presented in part B, the choices that were made and how the work was organized. 
Fig. 13 Detailed electrical and optical interfaces between the Mast Unit, the Body Unit, and the Rover. Some acronyms are used: GSE (Ground Support Equipments), C\&DH (Command and Data Handling), PRT

(Platinum Resistance

Thermometer)

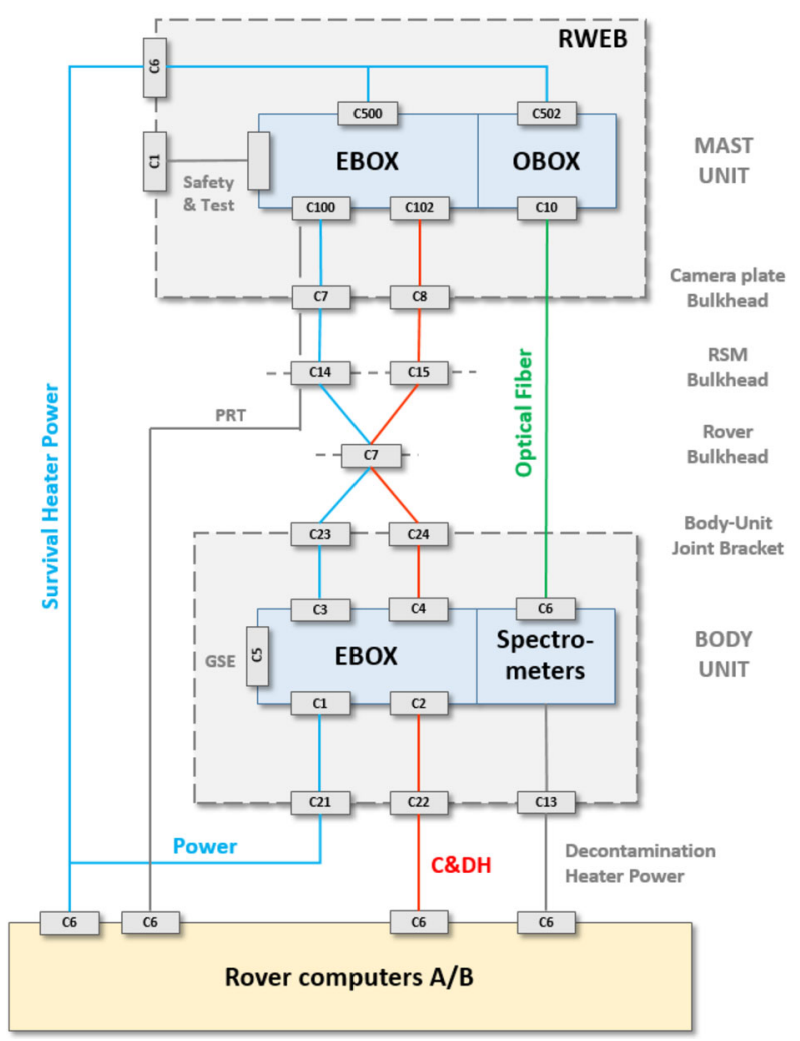

\subsection{System Overview}

The Mast Unit is made of two separate units - the Electronics Box (EBOX) and the Optical Box (OBOX) - that are electrically connected with each other (Fig. 14). The OBOX is built around a Schmidt-Cassegrain telescope that acts as an optical bench, on which several sub-systems are attached: a two-color laser and its Galilean beam expanders, a Continuous Wavelength Laser (CWL), and a translation unit for focusing. The optical bench also carries an imager, an infrared spectrometer, and the microphone. The EBOX contains several boards, including the Digital Processor Unit (DPU). The DPU communicates with the Body Unit and returns the acquired data through external harnesses. An optical fiber also connects the OBOX to the Body Unit for the analysis of LIBS, VIS, and TRR/L signals. Finally, a power line from the Body Unit feeds the Mast Unit. Hence, the Mast Unit is self-contained (Fig. 15); it has electrical and optical interfaces with the Body Unit, but no mechanical or thermal interfaces with it.

Figure 16 lists the key and driving functions that need to be implemented in the Mast Unit (see also Sect. 3.3) and the investigations that are associated with it. There are three outward capabilities associated with each of the three laser beams, and eight inward (or collection) capabilities. This figure illustrates the hardware paths taken by each of them, with regard to the systems presented in Fig. 14. It shows the unique hardware status of the MIC, at least in its standalone mode (no LIBS). All other investigations require the telescope/focus/objectives to collect light. For this reason, the focus capability has been doubled to two independent autofocus methods, plus a manual focus procedure. Some functionalities only 


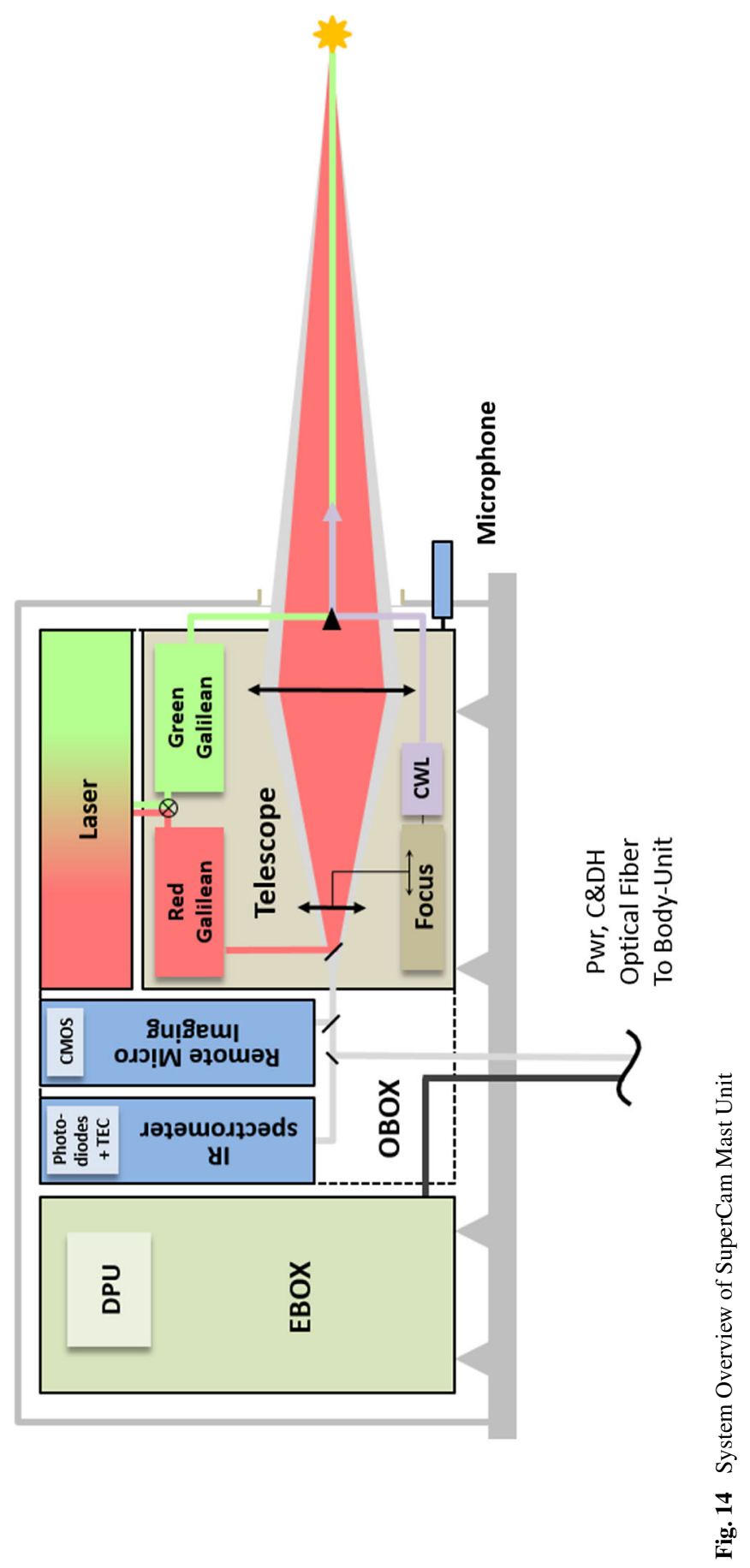



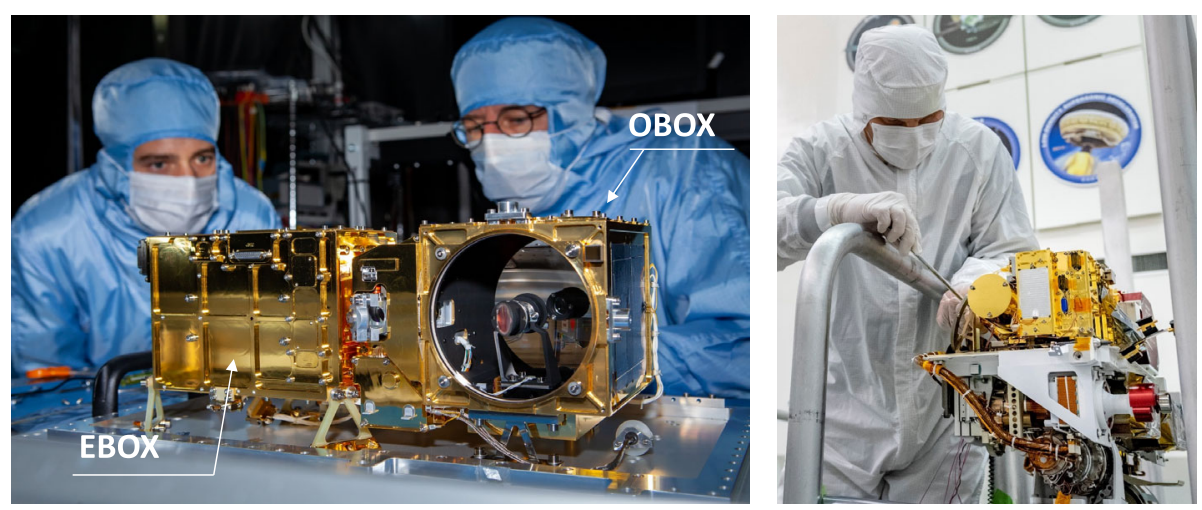

Fig. 15 (Left) Mast-Unit Flight Model in Toulouse, France. (Credit: CNES-TRONQUART Nicolas 2018). (Right) Integration on the rover camera bar at JPL (June 2019). (Credit: NASA/JPL-Caltech)

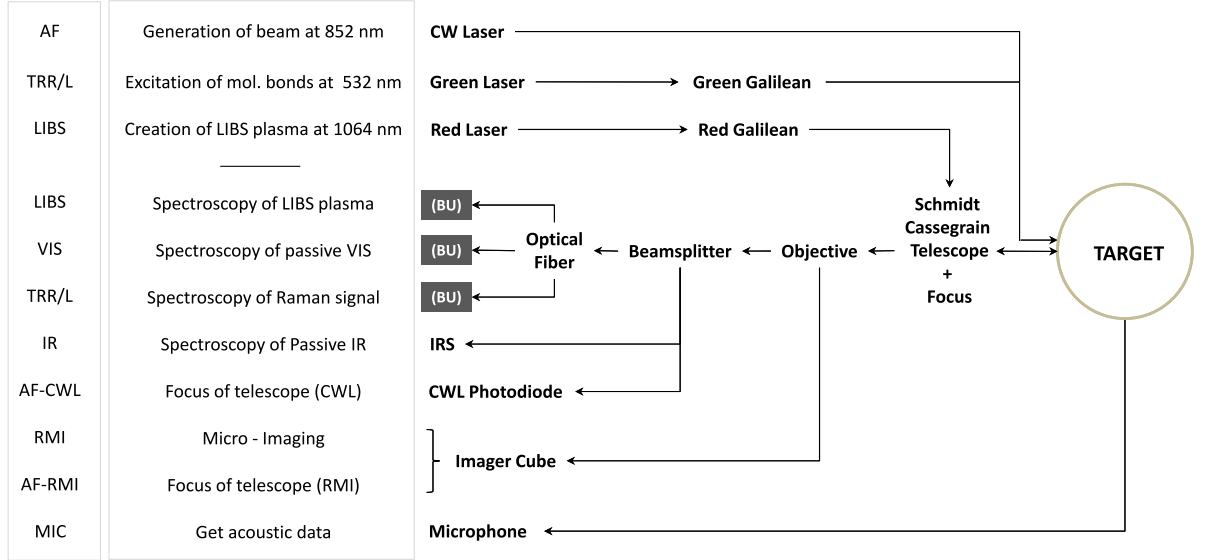

Fig. 16 Functional vs. hardware description of the Mast Unit. Left column, the science investigations and the autofocus capability (two methods). Mid column, the functions to implement in the Mast Unit. Right, the hardware paths to realize each investigation in the Mast Unit. The (optical) connection to the Body Unit is highlighted. See text (appendix) for the acronyms

need the Mast Unit to be realized, except for their control which always resides in the Body Unit. The quality of the signal injection into the fiber is then an essential characteristic of the instrument.

Once integrated, it becomes somewhat difficult to visually distinguish SuperCam from ChemCam (Fig. 11). The RWEB enclosure is the same on both rovers. An attentive eye will notice the SuperCam microphone, and a mirror in the center of the Schmidt window. Inside, while the overall architecture stays the same, most things changed, including the RMI and the LIBS laser, the translation stage and its motor that guides the secondary mirror. The Mast-Unit volume is the same as that of ChemCam, (H) $163 \mathrm{~mm}$, (L) $383 \mathrm{~mm}$, (W) $201 \mathrm{~mm}$, while the mass has increased slightly from $5.778 \mathrm{~kg}$ to $6.108 \mathrm{~kg}$. 


\subsection{Model Philosophy}

The philosophy of the models meets the requirements set out in the NASA Subcontract Data Requirements List (SDRL) to build and deliver at least an Engineering Development Unit (EDU), later used as a Testbed Unit (TU), and a Flight Model (FM). Besides those, the project also requires a robust Flight Spare (FS) strategy, either a complete model or spare parts. On top of that, SuperCam being an innovative instrument with limited heritage (as explained above), the team also decided to build an Engineering Qualification Model (EQM) to mitigate schedule, integration, and tests risks. A Structural and Thermal Model (STM) was also developed at an early stage of the project. Hence, we ended up very close to a conventional approach for innovative space instruments. In chronological order, these were the STM, EDU, EQM, and FM, and spare parts for the FS. Also, some simulators were developed to simulate the Mast-Unit/Body-Unit communication, to evaluate the timing of algorithms and so on.

\subsubsection{Structural and Thermal Model (STM)}

The STM was developed very early to verify the flight-readiness of the mechanical structure and thermal design. It is a mechanical and thermal simplified model, with no optics or subsystems, where electronic boards are populated with heaters to simulate power consumption and dissipation. This model passed vibration and shock tests at qualification levels to check on the various technical choices and to adjust model parameters. It also went through thermal tests ( 7 mbar of $\mathrm{N}_{2}$ ) to check the survival energy, thermal behavior during operations and robustness in Sun-safety configuration (see Sect. 4.10.2). As for the mechanical tests, data were used to adjust numerical models. Cumulatively, the STM was shocked and vibrated more than one hundred times during sub-system qualifications, and it spent weeks in a thermal chamber under image stereoscopy to test several mounting systems for the Schmidt plate.

\subsubsection{Engineering Development Unit/Testbed Unit (EDU/TU)}

Started in 2015, this first model, called the EDU, was built from ChemCam spare parts (Schmidt plate, primary and secondary mirrors, focus stage) and new parts as a proof of concept for TRR/L. This early model incorporated an infrared spectrometer connected via an optical fiber and a CMOS detector mounted on a printed circuit board (PCB) in place of the image cube. The EDU was operated in air at room temperature. In 2016, this unit was connected to an equivalent model of the Body Unit at LANL to conduct the first end-to-end optical tests and verification of the Mast-Unit-to-Body-Unit timings at nanosecond scales. This model was also used to investigate Raman background noise, and to study the potential of time-resolved luminescence more rigorously with the planned SuperCam configuration.

Later the EDU was refurbished into the TU, removing the laser (for safety purposes) and the IRS. Most functions are only simulated within the DPU, except the imaging capability. This model was delivered to JPL in 2017. Since then, it has been extensively used on the Vehicle System Test Bed (VSTB) to test commands and data handling with the rover computer. It also helped to refine the close loop pointing strategy (see Sect. 3.4) and develop the new intelligent software for autonomous targeting on Mars (AEGIS, Francis et al. 2017). 


\subsubsection{Engineering Qualification Model (EQM)}

The EQM is based on the final optical design, with critical subsystems (laser, infrared spectrometer, focus stage, microphone, shutter, imager cube) identical to the flight units and coming from the same lot. The EQM is fully functional, except for electronic components that are fit, form, and function, but not procured as space grade. It passed all environmental tests (vibration, shock, thermal vacuum, EMC/EMI) at "qualification levels", hence qualifying basically all Mast-Unit technologies and technical solutions. Thanks to this EQM, the flight unit could pass the same tests at "acceptance levels" (lower excitation levels than for qualification), except for shock and susceptibility that were not needed at acceptance level. Started in 2016, its integration and tests went on for about one year, followed by environmental tests for several weeks, and finally, the calibration of the infrared spectrometer. This model was also intensively used to validate the instrument flight software at different steps of the project, including the final version. It was delivered to LANL in 2018 for a series of coupling tests with the Body Unit that lasted several months, see Wiens et al. (this journal).

\subsubsection{Flight Model (FM)}

The FM is now installed on top of Perseverance rover Mast (Fig. 10). It has benefited a lot from the EQM developments. Overall it is identical to the EQM but with space grade EEE parts. Thanks to the EQM, the team had gained experience for its integration and tests. It was tested at acceptance levels (half the duration or levels of EQM's) for vibrations and thermal vacuum. Started in 2018, the FM model was completed in May 2019. As the flight model of the Body Unit had already been delivered to JPL, the FM Mast-Unit was coupled with the EQM Body-Unit for final software tests at IRAP, and limited cross-calibrations. Prior to this time, the EQM Mast-Unit had been used at LANL to conduct similar tests, plus extensive calibrations (see Wiens et al., this journal), with the Body-Unit flight model. Before leaving France, a full IR calibration was performed at the Laboratoire d'Etudes Spatiales et d'Instrumentation en Astrophysique (LESIA). The Mast Unit was delivered to JPL in June 2019 , and integrated on the rover shortly thereafter.

\subsubsection{Spare Strategy}

The decision was taken not to integrate a full FS, but to rely on critical spare parts. Once the Mast Unit is installed on the rover, in the RWEB, the main risk is at the interface level, i.e. within the electronics. Therefore, a full spare EBOX was built and qualified, while spare kits were procured for all the OBX sub-systems, but not integrated on a spare OBOX structure. If any spare part, including the EBOX, had to be used, extra compatibility tests with the rest of the instrument would have been necessary.

\subsection{Integrated Subsystems}

Some subsystems have been developed by independent teams, and were tested and delivered to the Mast-Unit team as complete subunits. Often, they have followed their own qualification path in parallel to other activities. These are the pulsed laser developed by Thalès Optronics (Elancourt, France) under the authority of CNES; the Imager cube by 3D+ (Buc, France) under the authority of CNES; the Infrared Spectrometer (IRS) by LESIA, France, which was also a partner of the whole instrument development; the focus mechanism by PI miCos (Germany) under the authority of CNES; the red beam shutter by CNES and LESIA, France; the microphone by ISAE-Supaéro, France. 
(a)

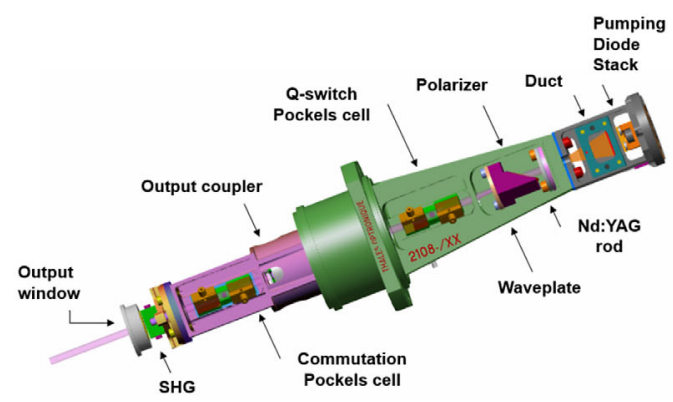

(b)
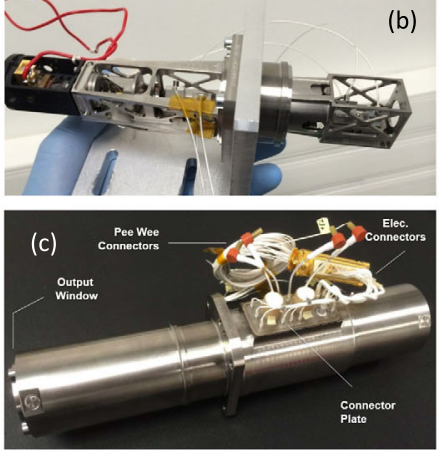

Fig. 17 SuperCam pulsed laser. (a) Details of inner parts. (b) Flight-unit before closure. (c) Flight-unit after closure. (Credit: Thales Optronics). The laser is $23 \mathrm{~cm}$ long

\subsubsection{Pulsed Laser}

The laser is a compact active Q-switched unit developed especially for SuperCam. This laser is composed of an oscillator, formed by a resonant cavity which is closed by two mirrors: a reflective mirror deposited on a Nd:YAG crystal on one end and a partially reflecting mirror (coupler mirror) on the other end. A multi-color diode stack pumps the crystal via a duct. The trigger system consists of a Pockels cell (or Q-switch), a polarizer, and a quarter waveplate to generate a short pulse. A second harmonic generator, commuted by a second Pockels cell at the output of the oscillator, can double the frequency of the beam (Fig. 17a). All optical components are mounted on a rigid Ti structure. A window closes the laser (Fig. 17b). The whole cavity is sealed to mitigate the risk of contamination of the optics, with a getter to absorb pollutants (Fig. 17c).

SuperCam's laser is an evolution from ChemCam's laser, with a few critical differences: (1) A more "classic" Nd:YAG crystal (fundamental emission at $1064 \mathrm{~nm}$ ) replaces the "exotic" Nd:KGW crystal (emission at $1067 \mathrm{~nm}$ ) used on ChemCam for the oscillator. The former is easier to procure, easing development cost, risk, and schedule. It can sustain higher heat fluxes, thus enabling the $10 \mathrm{~Hz} / 1000$ shot mode, whereas the same number of shots was validated on ChemCam at $3 \mathrm{~Hz}$ only. Simultaneously, the beam quality is improved, with an $\mathrm{M}^{2}$ of 1.2-1.5 for a better focus vs. a 1.4-2.5 range on ChemCam. (2) One $1800 \mathrm{~W}$ multi-color diode stack replaces the three $750 \mathrm{~W}$ pumping diode stacks on ChemCam. Its emission wavelength $(795-805 \mathrm{~nm})$ covers a wide range to cope with the narrower spectral acceptance of the Nd:YAG crystal and its variations over temperature. (3) The total energy is pumped by a single diode into the oscillator on SuperCam while half of the energy on ChemCam was produced in the oscillator by one diode, then amplified by the two amplifier stages, each one pumped by one diode. This ChemCam architecture could not be reproduced for SuperCam or the laser would have been too long for the allocated room on the rover mast. As a result, there is much more energy in the pumping cavity for SuperCam, and its stability has to be handled more carefully. (4) A Second-Harmonic Generator (SHG) was added to convert the $1064 \mathrm{~nm}$ light into $532 \mathrm{~nm}$ for TRR/L. The conversion between $1064 \mathrm{~nm}$ and $532 \mathrm{~nm}$ is performed by a second Pockels cell of the same kind as used for the Q-switch. For simplicity, the beam at $1064 \mathrm{~nm}$ is called the "red" beam, by analogy to the "green" beam at $532 \mathrm{~nm}$. In the green mode, the laser still emits a residual beam at $1064 \mathrm{~nm}$, since the SGH conversion efficiency is never $100 \%$; this residual beam is stopped 
Table 3 Summary of SuperCam laser characteristics. With an asterisk $(*)$ the range corresponds to the variations over the operating range

\begin{tabular}{ll}
\hline Type & Q-switch \\
Repetition rate & $3-10 \mathrm{~Hz}$ \\
Wavelength & $1064 \mathrm{~nm} \& 532 \mathrm{~nm}$ \\
Operating range & {$\left[-40^{\circ} \mathrm{C} ;+30^{\circ} \mathrm{C}\right]$} \\
Performance range & {$\left[-30^{\circ} \mathrm{C} ;+10^{\circ} \mathrm{C}\right]$} \\
Burst capacity & $1-1,000$ shots \\
Lifetime on Mars & $>10^{6} \mathrm{shots}(\mathrm{R}),>1.5 \times 10^{6}(\mathrm{G})$ \\
Energy & $>24 \mathrm{~mJ}(\mathrm{R}),>12 \mathrm{~mJ}(\mathrm{G})$ \\
Stability on energy & $<3 \%(\mathrm{short} \mathrm{bursts}),<10 \%$ long bursts) \\
Pulse duration* & $3-4 \mathrm{nsec}$ \\
Beam diam.* & $2.3-3.3 \mathrm{~mm} @ 1 / \mathrm{e}^{2}$ \\
Near field ellipticity* & $0.70-0.80$ \\
M & $<1.6(\mathrm{R})$ \\
Wavelength stability* & $2.4 \mathrm{pm} /{ }^{\circ} \mathrm{C}(\mathrm{G})$ \\
Polarization & $99.4 \%(\mathrm{G})$ \\
Jitter & $\sim 5 \mathrm{nsec}$ \\
Pointing stability* & $<0.4 \mathrm{mrad} \mathrm{EL},<0.15 \mathrm{mrad} \mathrm{AZ}$ \\
Off-axis stability* & $<100 \mu \mathrm{m}$ \\
Mass & $580 \mathrm{~g}$ \\
Length x Diam. & $230 \mathrm{~mm} \times 48 \mathrm{~mm}$ \\
\hline
\end{tabular}

by the shutter. On the other hand, in the red mode, we observe a residual green beam due to a non-perfect polarization alignment between the oscillator output and the frequency doubling crystal. The few millijoules of green light are not focused on the target and have no effect on the LIBS spectra. Other characteristics contributing to the success of the ChemCam laser remain unchanged: $\sim 3 \mathrm{~mm}$ output beam diameter, sealed cavity with getter, mounting ring, Ti structure, passive cooling, internal heaters, and connector plate. The mass and volume resources (SuperCam $580 \mathrm{~g}$ vs. $550 \mathrm{~g}$ ChemCam, length $230 \mathrm{~mm}$ vs. $214 \mathrm{~mm}$, diameters unchanged) are similar while offering better performance, and extended operational modes. See Table 3 for a summary of the laser characteristics.

Key and driving requirements for SuperCam laser (at its exit aperture) are: $>24 \mathrm{~mJ}$ in red, $>12 \mathrm{~mJ}$ frequency doubled into green, beam size (as defined by $1 / \mathrm{e}^{2}$ of the near field profile) between 2.5 and $3.5 \mathrm{~mm}$, beam quality $\mathrm{M}^{2}<2$ in red, and pulse duration $<5 \mathrm{~ns}$. The laser was qualified to one million red shots and 1.5 million green shots.

The energy output and beam quality depend a lot on the temperature of the laser body. The laser is qualified for surviving from $-50{ }^{\circ} \mathrm{C}$ to $+55^{\circ} \mathrm{C}$, with a nominal operating range from $-40{ }^{\circ} \mathrm{C}$ to $+30{ }^{\circ} \mathrm{C}$. Performance meet the specifications from $-30{ }^{\circ} \mathrm{C}$ to $+10{ }^{\circ} \mathrm{C}$. The laser has no thermal regulation, but two heaters to bring it within the performance range. The power to heat the stack is $\leq 3.2 \mathrm{~W}$. At this value, it takes $\sim 5$ min to warm the laser stack from $-40{ }^{\circ} \mathrm{C}$ to $-15^{\circ} \mathrm{C}$. For the frequency doubling crystal, the heater power is $\leq 1.2 \mathrm{~W}$. At this value, it takes $\sim 4$ min to warm the laser from $-40{ }^{\circ} \mathrm{C}$ to $-10^{\circ} \mathrm{C}$. Each time the laser is used, if needed, the stack and doubler temperatures will be raised to $-15^{\circ} \mathrm{C}$ and $-10{ }^{\circ} \mathrm{C}$, respectively: this occurs for operations at nighttime and morning, as long as the instrument temperature is below these limits.

The laser can perform single shots, but most often "bursts" are used: a burst is a consecutive series of laser shots. Bursts are typically $20-50$ shots at $3 \mathrm{~Hz}$ for LIBS but this can go up to 500 for depth profiles. Bursts for TRR/L are usually 100-200 shots (at 3 or $10 \mathrm{~Hz}$ ) but this can also go up to 1,000 shots (sequence of 5 bursts of 200 shots, with $30 \mathrm{~s}$ rest time between each burst). When the laser was optimized, a standard 100 shots $/ 3 \mathrm{~Hz}$ in red and 100 
Fig. 18 Laser energy versus pumping current at different temperatures of the laser. (a) Red laser beam, burst of 20 shots at $3 \mathrm{~Hz}$. The dashed line corresponds to the requirement, $24 \mathrm{~mJ}$. (b) Green laser beam, burst of 20 shots at $3 \mathrm{~Hz}$. The dashed line corresponds to the requirement, $12 \mathrm{~mJ}$
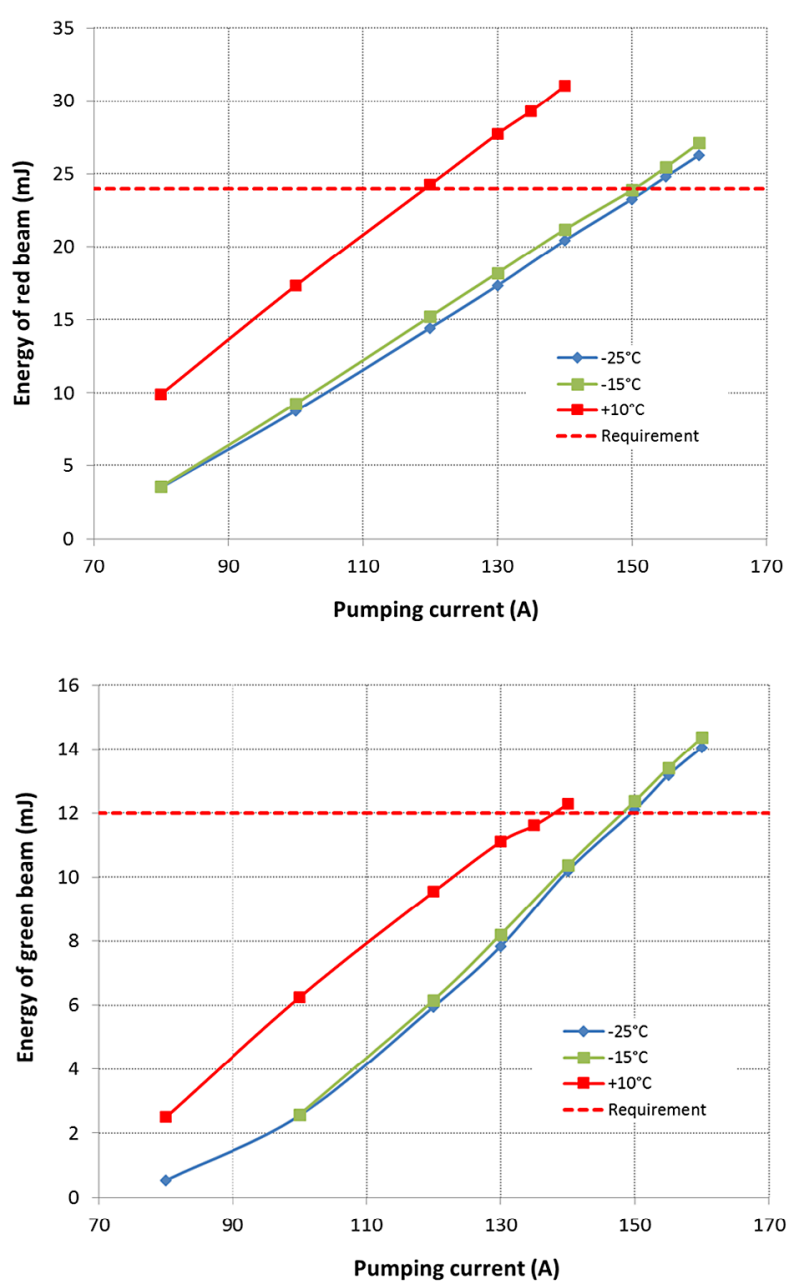

shots/10 Hz in green was used with laboratory electronics, i.e., the "short" burst mode. The performance was later checked with 20 shots only and with the laser board in a flight-like configuration.

To operate the laser within its nominal range, the pumping current of the diode stack is the only readily adjustable parameter. Using the short burst mode, the output energy at different temperatures of the laser is measured for various currents. During these tests, the laser stack and crystal doubler are maintained above their respective thresholds at $-15{ }^{\circ} \mathrm{C}$ and $-10{ }^{\circ} \mathrm{C}$, respectively. However, the performance is also a function of the laser external temperature, which will be driven by the environment and instrument temperatures on Mars. Figure 18 shows the energy of the red and green beams at the output of the laser at three temperatures. As the current ramps up, the energy increases as expected for all temperatures. Therefore, for each temperature of the laser, there is a current threshold to achieve the key energy requirements. It requires more current below $-15^{\circ} \mathrm{C}$ than at $10{ }^{\circ} \mathrm{C}$ to reach the performance requirements because of a temperature-induced change of the wavelength distribution of the multi-color diode stack with respect to the absorption of the YAG crystal. The set point in current should not be much above the threshold in order to minimize heating 
Table 4 Maximum stack current as a function of the laser temperature, as a start of an activity. In red, the range where requirements on energy are specified and met

\begin{tabular}{|c|c|c|c|c|c|}
\hline $\begin{array}{l}\text { Laser } \\
\text { Temp. }\end{array}$ & $\begin{array}{c}\text { Stack } \\
\text { Current }\end{array}$ & $\begin{array}{l}\text { Stack } \\
\text { Temp. }\end{array}$ & $\begin{array}{c}\text { Doubler } \\
\text { Temp. }\end{array}$ & $\begin{array}{l}\text { “Red" } \\
\text { Energy }\end{array}$ & $\begin{array}{l}\text { "Green" } \\
\text { Energy }\end{array}$ \\
\hline $25^{\circ} \mathrm{C}$ & $140 \mathrm{~A}$ & $25^{\circ} \mathrm{C}$ & $25^{\circ} \mathrm{C}$ & $\geq 25 \mathrm{~mJ}$ & $\sim 4.5 \mathrm{~mJ}$ \\
\hline $10^{\circ} \mathrm{C}$ & \multirow{3}{*}{$140 \mathrm{~A}$} & $10^{\circ} \mathrm{C}$ & $10^{\circ} \mathrm{C}$ & $\sim 30 \mathrm{~mJ}$ & $\sim 12 \mathrm{~mJ}$ \\
\hline $0^{\circ} \mathrm{C}$ & & $0^{\circ} \mathrm{C}$ & $0^{\circ} \mathrm{C}$ & $>28 \mathrm{~mJ}$ & $>15 \mathrm{~mJ}$ \\
\hline$-10^{\circ} \mathrm{C}$ & & $-10^{\circ} \mathrm{C}$ & \multirow{4}{*}{$-10^{\circ} \mathrm{C}$} & $>25 \mathrm{~mJ}$ & $>13 \mathrm{~mJ}$ \\
\hline$-15^{\circ} \mathrm{C}$ & \multirow{3}{*}{$155 \mathrm{~A}$} & \multirow{4}{*}{$-15^{\circ} \mathrm{C}$} & & $>24 \mathrm{~mJ}$ & $\sim 13 \mathrm{~mJ}$ \\
\hline$-20^{\circ} \mathrm{C}$ & & & & $>24 \mathrm{~mJ}$ & $>13 \mathrm{~mJ}$ \\
\hline$-30^{\circ} \mathrm{C}$ & & & & $\sim 24 \mathrm{~mJ}$ & $\sim 12 \mathrm{~mJ}$ \\
\hline$-40^{\circ} \mathrm{C}$ & & & & $>22 \mathrm{~mJ}$ & $>12 \mathrm{~mJ}$ \\
\hline
\end{tabular}

inside the cavity. At the same time, we want to minimize the number of modes, which have to be programmed onboard. Table 4 shows a compromise for the currents used for the flight laser. Above $10^{\circ} \mathrm{C}$, the laser cannot reach $12 \mathrm{~mJ}$ for the green beam because of a reduced efficiency of the doubling crystal over this temperature range. Below $-30{ }^{\circ} \mathrm{C}, 24 \mathrm{~mJ}$ is difficult to reach for the red beam because of poor coupling between the pumping stack and the YAG crystal.

Beyond the short burst mode, the science team has defined realistic sequences of alternatively red and green laser bursts for raster activities, at $3 \mathrm{~Hz}$ for LIBS and $10 \mathrm{~Hz}$ for TRR. Between two consecutive bursts, there is always a $30 \mathrm{~s}$ pause. When the same activity is repeated several times, a 3 min pause is inserted.

- Sequence \#1 (5 point raster of LIBS/Raman): $5 \times(30$ red + 200 green), 4 times

- Sequence \#2 (10 points LIBS): $10 \times(50$ red), 4 times

- Sequence \#3 (10 point raster of LIBS/Raman): 10× (30 red + 200 green), 2 times

- Sequence \#4 (5 point raster of Raman only): $5 \times$ (150 green), 3 times

- Sequence \#5 (2 point raster of LIBS depth profile): $2 \times(500 \mathrm{red}), 1$ time

- Sequence \#6 (10 point raster of LIBS/Raman): $5 \times(2 \times 30$ red +200 green $), 4$ times

- Sequence \#7 (10 point raster of Raman only): 10× (200 green), 1 time

The purpose of these tests was to make sure that the laser stays stable (within margins) under these energy loads for complex activities that are repeated several times. Figure 19 shows the laser energy for sequence $\# 5$ at $-25^{\circ} \mathrm{C}$ and a total of 1,000 shots at $3 \mathrm{~Hz}$. After 500 shots, the laser has warmed up and becomes more efficient, gaining $\sim 1 \mathrm{~mJ}$. There is a $30 \mathrm{sec}$ pause before the next burst of 500 shots. Figure 20 shows the laser energy for sequence \#7 at $-25^{\circ} \mathrm{C}$ and a total of 2,000 shots at $10 \mathrm{~Hz}$. After each burst of 200 shots, the laser energy increases by $\sim 1 \mathrm{~mJ}$. In this case, like in the previous one, the $30 \mathrm{sec}$ pause between bursts does not reset entirely the energy profile. To make sure that these sequences are safe, they have been played several times to evaluate margins.

Figure 21 compares average energies for short and long bursts, especially across the $-15^{\circ} \mathrm{C}$ boundary. For the red beam, the results are comparable, but for the green beam, long rasters produce more energy, since the crystal warms up and the pumping becomes more efficient (see also Fig. 19). As expected, requirements in energy are met, but the set point is difficult to establish around $-15^{\circ} \mathrm{C}$, where the maximum pumping current decreases from $155 \mathrm{~A}$ to $140 \mathrm{~A}$. Another important parameter (not shown) for LIBS is the stability of the red beam in energy: it is better than $3 \%$ RMS over 100 shots.

Several other parameters have been measured with temperature. In the near field, the beam at the laser output has an elliptical shape, with an aspect ratio between 0.7 and 0.8 . The red laser beam is slightly larger than the green beam: $2.7 \mathrm{~mm} \times 3.3 \mathrm{~mm}$ on average over the performance range, versus $2.3 \mathrm{~mm} \times 3 \mathrm{~mm}$ for the green. Both increase slightly at 


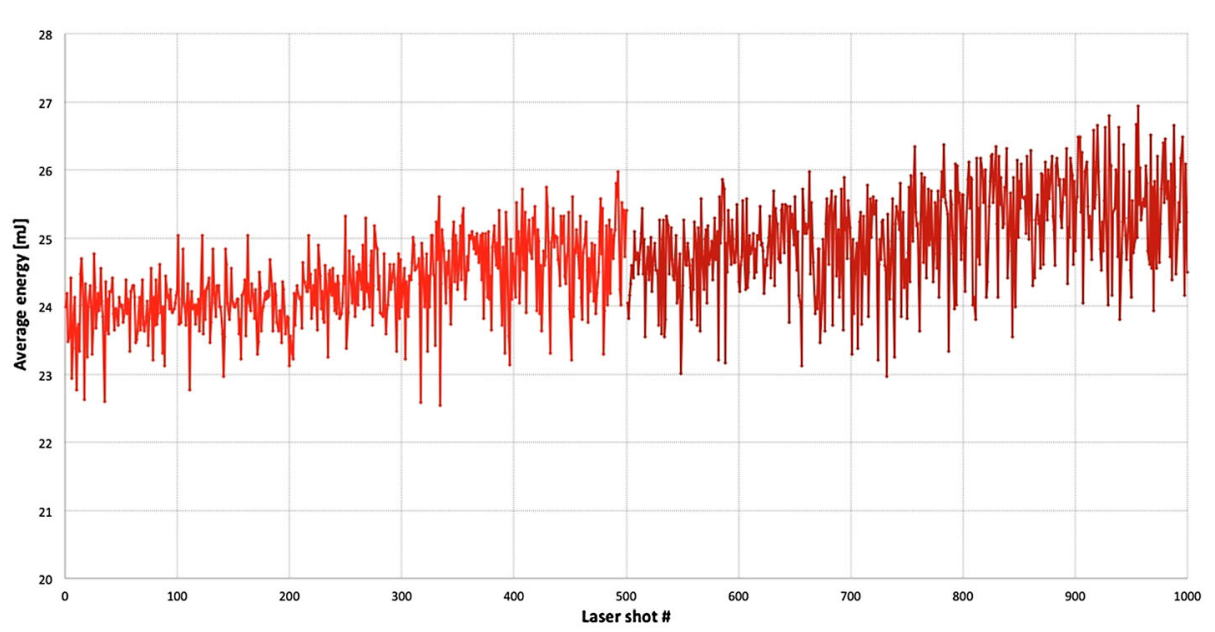

Fig. 19 Laser energy at $1064 \mathrm{~nm}, 3 \mathrm{~Hz}$, for sequence \#5, at $-25^{\circ} \mathrm{C}$. The pumping current is $155 \mathrm{~A}$. The requirement is $22 \mathrm{~mJ}$

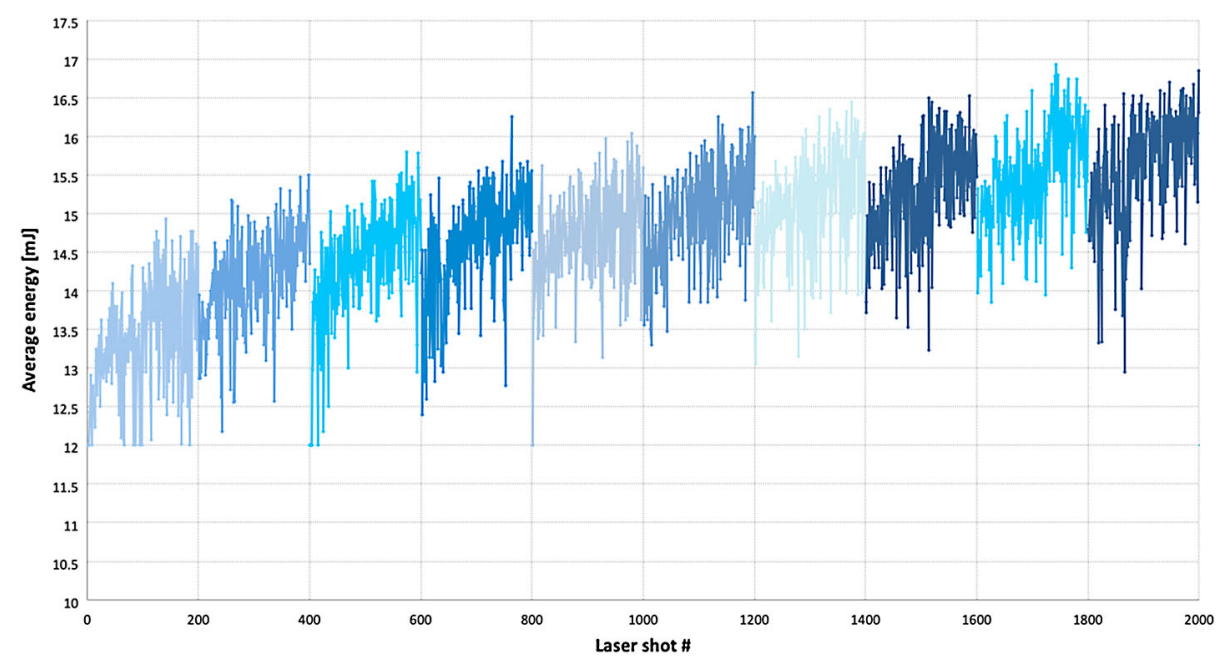

Fig. 20 Laser energy at $532 \mathrm{~nm}, 10 \mathrm{~Hz}$, for the sequence \#7, at $-25^{\circ} \mathrm{C}$. The pumping current is $155 \mathrm{~A}$. The requirement is $12 \mathrm{~mJ}$

cold temperatures by $\sim 0.5 \mathrm{~mm}$. The pulse duration is mostly essential for LIBS: it varies between $3.4 \mathrm{~ns}$ and $3.9 \mathrm{~ns}$ with temperature. The green pulse duration is typically $0.5 \mathrm{~ns}$ shorter. The beam quality $\left(\mathrm{M}^{2}\right)$ is the main characteristic of this laser, as it is essential to focusing the red beam: 1.4 at $-30{ }^{\circ} \mathrm{C}, 1.3$ at $-10{ }^{\circ} \mathrm{C}$. Accuracy and stability of the wavelength of the green emission is essential for Raman spectroscopy. It was calibrated with temperature (Fig. 22). It varies by a tenth of a nanometer $\left(3.5 \mathrm{~cm}^{-1}\right)$ over the performance range. For the worst-case (sequence \#7), the green wavelength varies by less than $0.04 \mathrm{~nm}$ $\left(1.3 \mathrm{~cm}^{-1}\right)$ during an activity. Table 3 displays more characteristics, especially the pointing stability over the performance range, which helps keep the system aligned. 


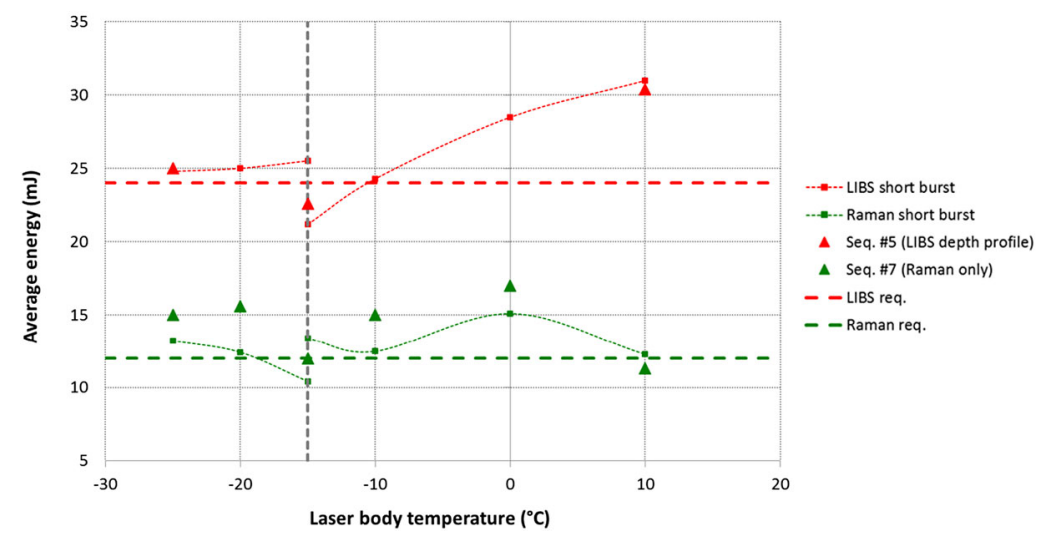

Fig. 21 Average laser energy versus temperature for 20 short bursts and long bursts (sequences \#5 and \#7). Dashed lines represent the requirements at $1064 \mathrm{~nm}, 24 \mathrm{~mJ}$ (red), and at $532 \mathrm{~nm}, 12 \mathrm{~mJ}$ (green)

Fig. 22 Variations of laser wavelength with temperature for the green beam

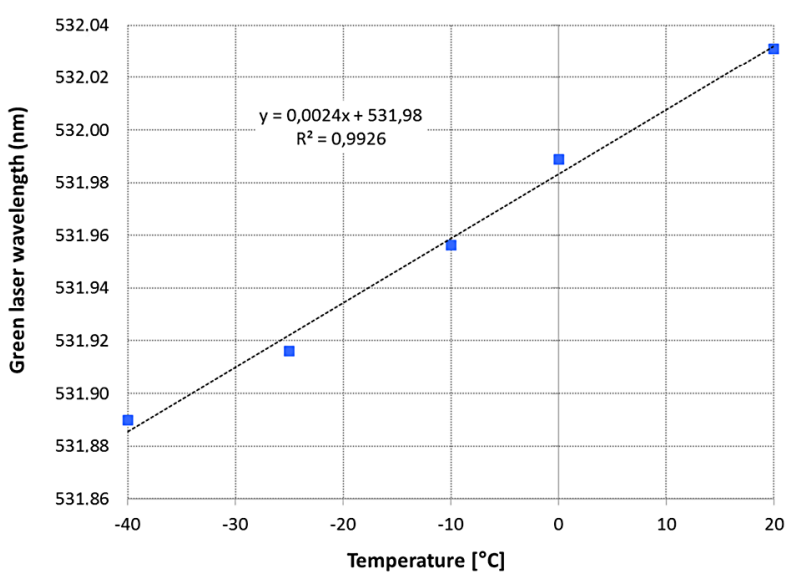

\subsubsection{Imager Cube}

RMI images will document all other SuperCam activities (LIBS, TRR/L, VISIR) by observing each target at high resolution to characterize grain sizes, shapes and colors: nodules, laminations, etc. The image resolution is sufficient to visualize the craters ablated by the laser up to $7 \mathrm{~m}$ and therefore to localize the source of each LIBS spectrum. The Body Unit collects for passive and TRR/L, and MU collects for IRS, can be localized in the RMI frame of reference since these activities are globally co-aligned. The distance range can be extended for VISIR and is only limited by atmospheric transparency and the horizon. As a stand-alone capability, the RMI imager will provide long-range high-resolution color images for mosaics that can be integrated into Mastcam-Z images, which have a much larger field of view. RMI provides better resolved images than HiRISE on the Mars Reconnaissance Orbiter up to $5 \mathrm{~km}$ from the rover, and of course with a different perspective.

The RMI capability relies on the telescope to collect light, a dioptric objective to image the scene on the detector, and the detector itself combined with its front-end-electronics (3D cube). The main difference to the ChemCam RMI are the change of technology from the 
Fig. 23 Standard Bayer filter response of the CMOS CMV4000 RGB filters (CMOSIS data)

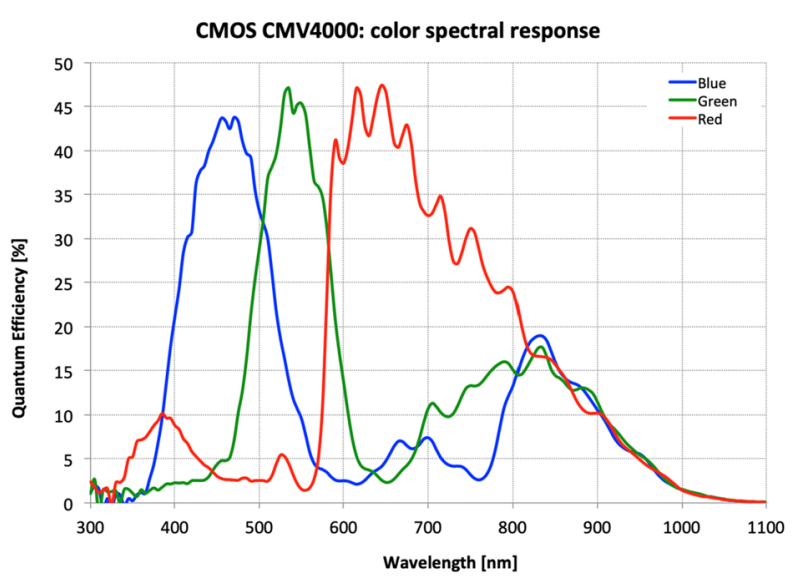

CCD to CMOS and the addition of color. The RMI alignment with respect to the telescope boresight was verified, and is stable at the $\pm 0.6 \mathrm{mrad}$ level over $70 \mathrm{~K}$.

The detector is an off-the-shelf low-noise complementary metal-oxide semiconductor (CMOS) image sensor CMV4000 by AMS CMOSIS (Antwerp, Belgium) consisting of $2048 \times 2048$ square pixels at $5.5 \mu \mathrm{m}$ pitch. A screening program was defined to select flightquality detectors, on which lot acceptance tests were performed to evaluate their tolerance to space and Mars environmental constraints. Dark current (hot pixels), per-pixel gain and linearity, pixel-to-pixel photon response non-uniformity were measured. Pixel values are coded on 10 bits, the linear part of the sensor ranges from 20 to $~ 900 \mathrm{DN}$. When HDR (High Dynamic Range) mode is used, images can extend to 13 bits by combining 7 frames. In all cases, images are coded in 16 bits.

Per-pixel micro-lenses were mounted during the CMOS encapsulation process to enhance light collection toward the photosensitive area itself. Bayer filters are also integrated in the process (Fig. 23). CNES has qualified the positioning of the filters on the detector for our application. An RMI image is therefore equivalent to four matrices of $1024 \times 1024$ pixels color filtered in red, green (two identical filters), and blue (RGB) bands. The relative response between these four filters was checked during the tests at component level.

The CMOS image sensor is mounted in an aluminum cradle, and then packaged by $3 \mathrm{D}$ plus (Buc, France) together with a 3 Mega-gate FPGA and a dedicated anti latch-up circuit (Fig. 24). This packaging is common to several space programs (Rosetta, Curiosity, ExoMars, Mars 2020, etc.). It is very compact, low mass, self-contained, and simple to test and integrate electrically. The cradle, and the position of the image sensor within the cradle itself, allow for an accurate positioning of the detector on the beamsplitter. For SuperCam, the internal FPGA is barely used, and it only controls the protection circuit against latch-up. Image processing is done in the Mast-Unit DPU. A flexible PCB connects the 3D cube to the instrument DPU and power boards.

It is not straightforward to add an imaging capability within a telescope that is designed to focus laser beams as small as possible. Several iterations and compromises led to a nottoo-small field of view (FOV) and high resolution (to visualize laser pits) over a large temperature range $\left[-40{ }^{\circ} \mathrm{C} ;+30^{\circ} \mathrm{C}\right]$ covering what is expected on Mars, plus ambient for Earth tests. $18.8 \mathrm{mrad}$ was reached for the FOV. Over this surface, the distortion is negligible $<2.25 \%$. The pixel iFOV is $\sim 10 \mu \mathrm{rad}$. Figure 25 illustrates the RMI FOV during System Thermal Tests at JPL using a $7.3 \mathrm{~cm}(\mathrm{~L})$ by $\sim 7.2 \mathrm{~cm}(\mathrm{H})$ rock at different distances. LIBS pits are visible on the dark clast, which is at the center of the image at $1.56 \mathrm{~m}$. At 

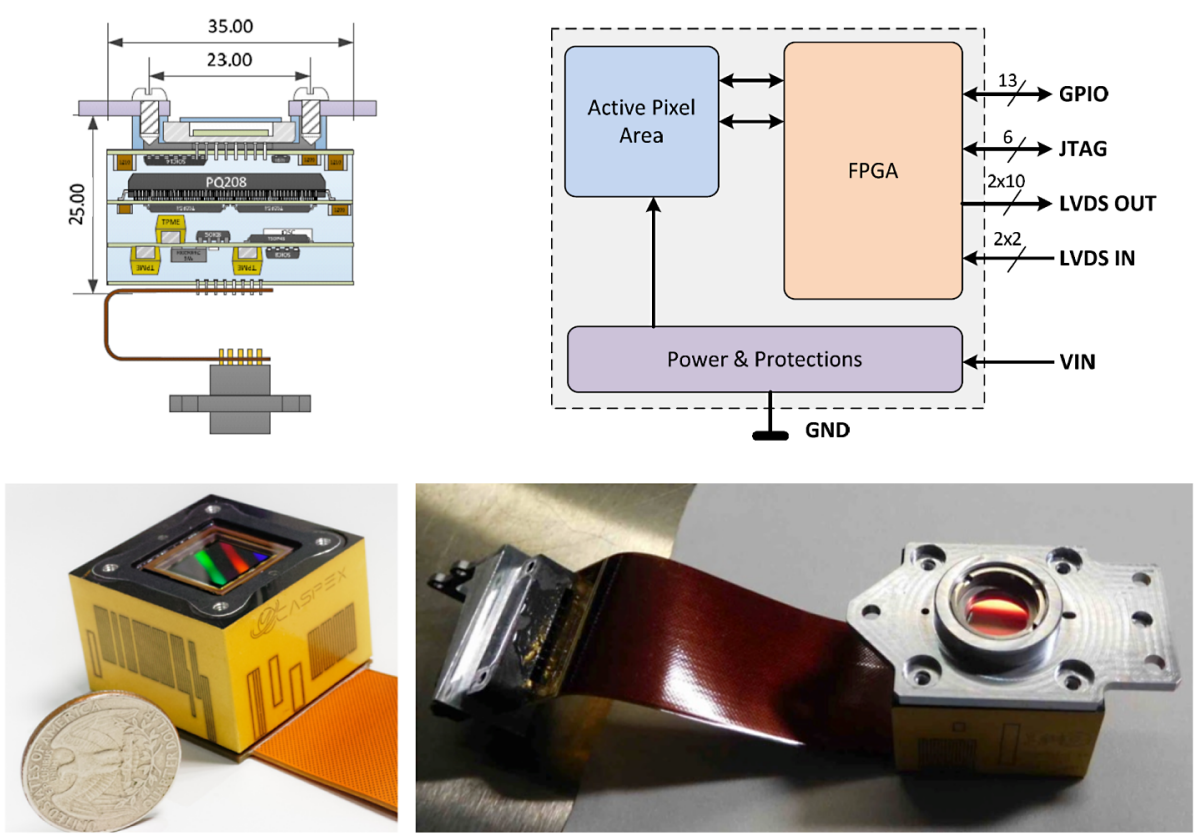

Fig. 24 Imager cube. (Top right) Overall architecture with input/output signals. (Top left) Internal layout and dimensions. (Bottom) Flight-unit with flex cable, the IR cut-off filter, and the structure to hold it on the Optical bench

$7 \mathrm{~m}$, the image captures the whole rock. At this distance, the FOV is $13 \mathrm{~cm}$ in diameter (it is slightly cropped in the figure). As shown by the last image in Fig. 25, the RMI can focus to infinity.

The telescope point spread function covers a $15-\mu \mathrm{m}$ area at the focal plane, therefore at least 4 pixels $(2 \times 2)$ of different colors. Hence, SuperCam achieves with color the same resolution as ChemCam whose pixel size was $14 \mu \mathrm{m}$. Measurements show a spatial resolution better than $80 \mu \mathrm{rad}$ with a contrast greater than $20 \%$ at 20 line pairs $/ \mathrm{mm}$ over half of the FOV (Fig. 26). At $80 \mu \mathrm{rad}$ the effective resolution is $1 / 4 \mathrm{~mm}$ at $3 \mathrm{~m}$. Figure 27 illustrates the high-resolution reached by RMI during the System Thermal Tests at JPL, at $-40{ }^{\circ} \mathrm{C}$. On the calibration target, the LIBS pit $(<500 \mu \mathrm{m})$ is clearly visible. Up to $4.4 \mathrm{~m}$, details smaller than $1 \mathrm{~mm}$ are visible. The main difficulty with high-resolution is the reduced Depth-OfField (DOF). The DOF corresponding to a loss of contrast of $20 \%$ is \pm 11 motor steps. This translates into $\pm 4 \mathrm{~mm}$ at $2 \mathrm{~m},-10.3 /+10.6 \mathrm{~cm}$ at $10 \mathrm{~m}$. While operating on Mars, a zstack acquisition can occasionally be planned to allow features from a larger depth range to be shown in focus (Wiens et al. this journal).

The spectral response of the RMI includes that of the RGB filters and that of the telescope and objective. Due to the sharing of the incoming light with the spectral sensors, only $10 \%$ of the light entering the telescope reaches the RMI detector. A low-pass cut-off filter was added at $650 \mathrm{~nm}$ to reject the near-infrared range, which would have distorted the Bayer colors. The resulting spectral response, including all optics, dichroics, and filters along the light path, is very different from ChemCam, whose main light collection for the RMI is in the NIR range (Fig. 28). We thus find that preliminary multiplicative factors to balance each color are 1 for Red, 1.01 for Green, 0.96 for Blue. By nature, Cassegrain telescopes have a large central obscuration that induces some vignetting of the light. Simulations and data 

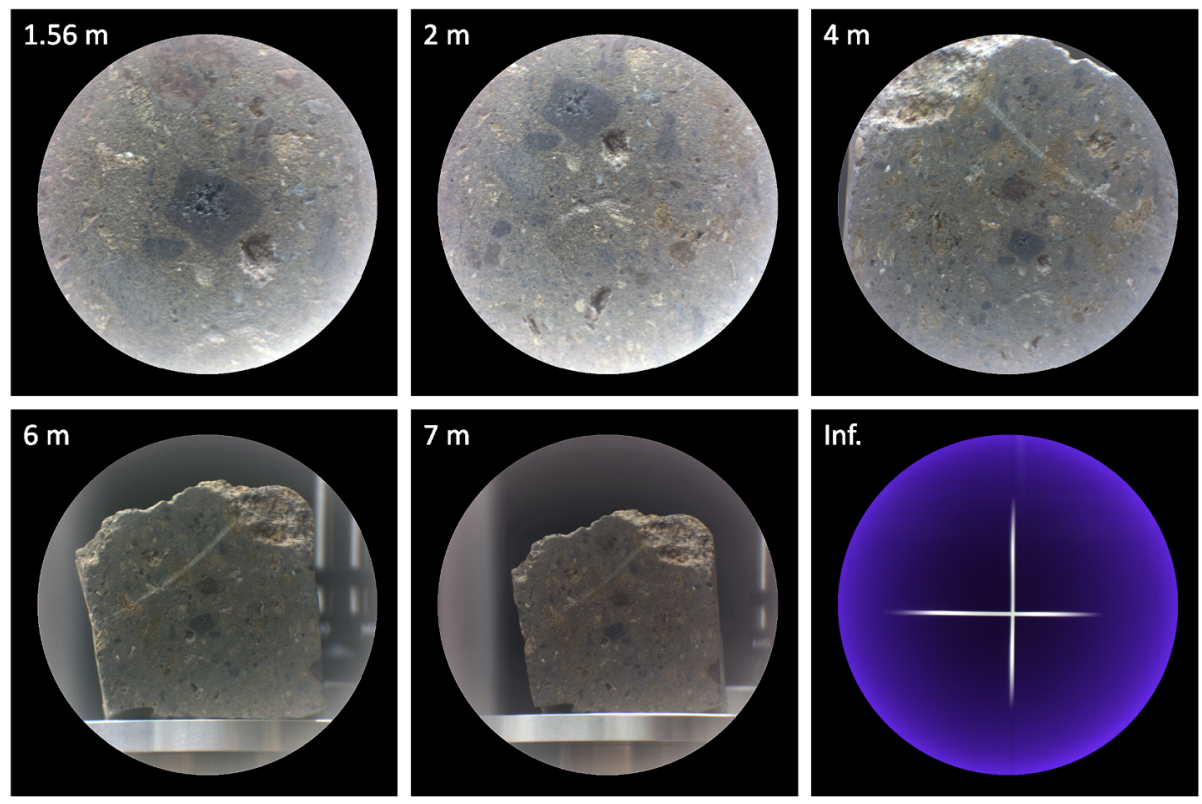

Fig. 25 Series of RMI images acquired at $-40{ }^{\circ} \mathrm{C}$ during the Mast-Unit thermal tests. The same rock is placed at different distances. Its size is $\sim 7.3 \mathrm{~cm}(\mathrm{~L}) \times \sim 7.2 \mathrm{~cm}(\mathrm{H})$. The dark crystal at the center of the first image measures $\sim 8 \mathrm{~mm}(\mathrm{~L})$ by $\sim 7 \mathrm{~mm}(\mathrm{H})$. LIBS pits can be seen. The last is an image of a collimator reticule that mimics infinity

show that the telescope vignetting decreases the flux on the sensor by a factor of 5 from the center to the edges of the circular FOV. However, noise reduces slower and thus the SNR decreases by only a factor of 2 . The accuracy of the flat field pixel-to-pixel is known to $\pm 5 \%$ at $20^{\circ} \mathrm{C}$. A refinement of the flat field will be acquired directly on Mars by using sky observations, as is done routinely for ChemCam. The FM telescope's internal baffle was optimized to reduce stray light. Stray light may still be visible in some configurations (focus at short distance, dark target, and bright source off the FOV) but it will be corrected during post-processing. There are no relevant ghost images.

A radiometric model shows that exposure times will range from 3 to $110 \mathrm{~ms}$ on Mars (0.4 albedo, midday). Typically, each pixel of a single image should reach a SNR of 80/60 on red/blue pixels, when using $80 \%$ of the full-well capacity of the red pixels. Binning the color pixels will double the SNR for monochrome images. The HDR mode can increase the full-well capacity by a factor of 5 before linearization. Hence an apparent SNR $>200$ at half dynamic for an irradiance of $300 \mathrm{~W} / \mathrm{m}^{2}$ (on target) and an albedo of 0.4 can be obtained. Radiometric calibration is not required for a context imager, but with the precise photon budget that was measured at instrument level, an accuracy better than $\pm 20 \%$ at $20{ }^{\circ} \mathrm{C}$ can be achieved.

During Assembly, Tests, and Launch Operations (ATLO) testing at JPL, a few (sub)millimeter foreign object debris (FOD) particles were identified on RMI images. They are not on the detector itself but most probably on the RMI dichroic or the IR cut-off filter (optics $\# 10$ and \#11 in Fig. 45). Altogether, they represent about $0.6 \%$ of the total surface, and at most a local decrease of $12 \%$ in intensity (generally less). Tests have shown that FOD signatures can be numerically removed during the flat field correction. A new flat field will be acquired as soon as possible after deployment on Mars to characterize those FOD. 
Fig. 26 RMI resolution. (Top) Contrast at the center of the field of view and at 5 mrad off the center. The requirement is in red. (Bottom) Contrast always at the center of the field of view for different temperatures
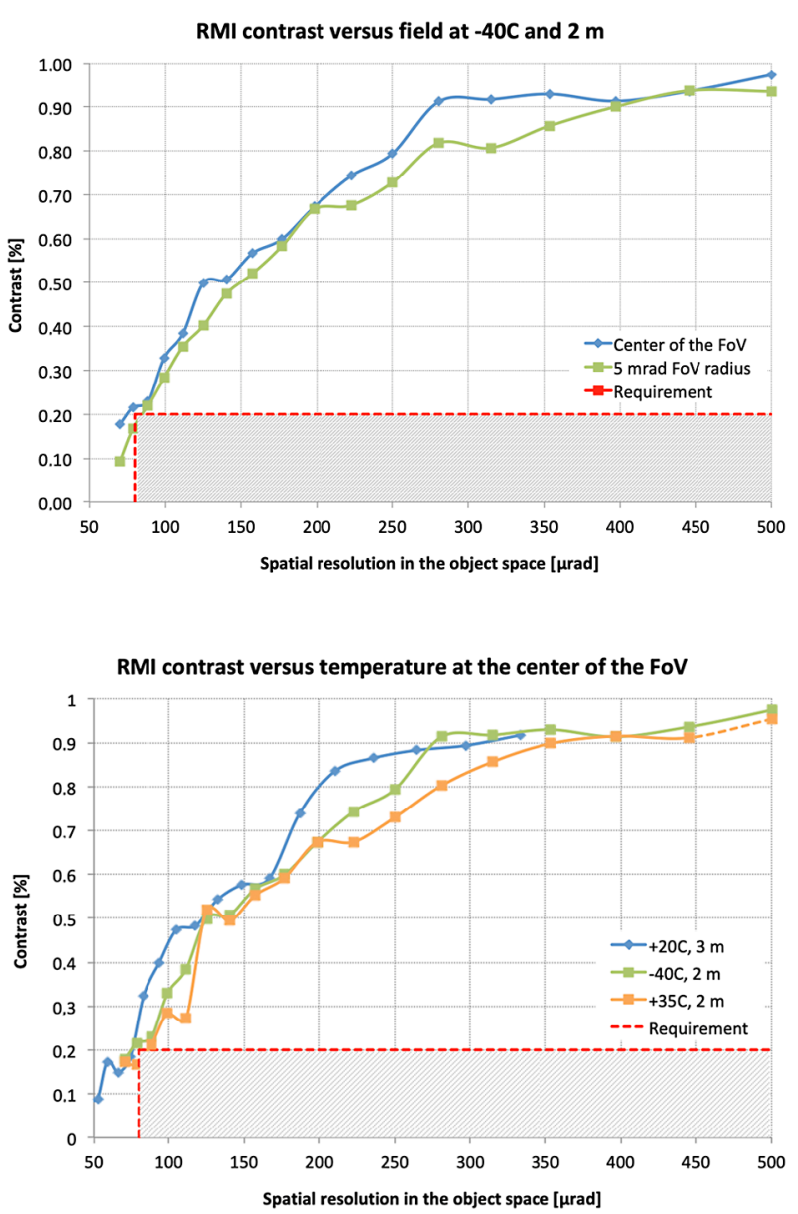

Several algorithms are associated with the RMI: auto-exposure for single-frame exposure time, autofocus for the telescope, z-stack to increase depth-of-field (DOF; Wiens et al. this journal), and HDR to increase SNR and dynamic range. These will be detailed in Sect. 4.9. To reduce data volume, the rover internal computer will compress RMI images without non-linear data transformation. Two options are available: compression with ICER at an adjustable bit rate (Kiely et al. 2007) or JPEG format with Malvar demosaicing and 8-bit scaling.

A few calibration targets are dedicated to checking RMI performance and adjusting HDR parameters: alumina with $\mathrm{Cr}$ features (USAF groups one to four, slanted-edge checkboard) to characterize resolution, Modulation Transfer Function (MTF), and focus quality; a grayscale target along with points of different sizes to check the HDR performance and point spread function; five color targets with magnets: Aluwhite (IR), Black paint (IR), Cyan, Red, and Green sintered ceramics. See Manrique et al. (this journal) for details. In addition to these targets, several calibrations are possible in flight: dark or star observations at night, flat field on the sky, cross calibration with MastCam-Z calibration targets. 

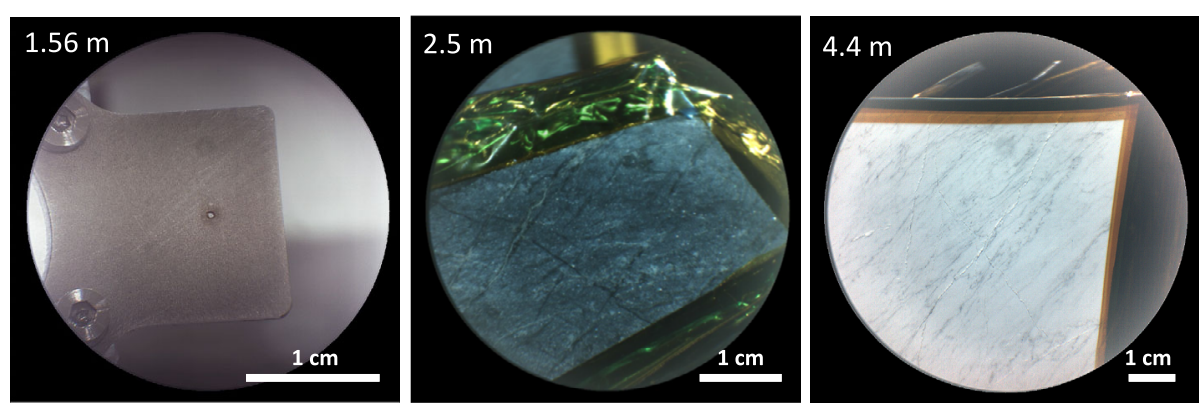

Fig. 27 Series of RMI images during System Thermal Tests at JPL. (Left) Ti calibration target. LIBS pit $\leq 0.5 \mathrm{~mm}$ diameter. (Middle) Composite target (62\% Ilmenite, 38\% Hematite) at $2.5 \mathrm{~m}$. (Right) Gypsum with some bassanite (black streaks are likely organics) at $4.4 \mathrm{~m}$. The two images on the right are acquired with the solar simulator on

Fig. 28 Overall transmission to the RMI, and comparison to ChemCam

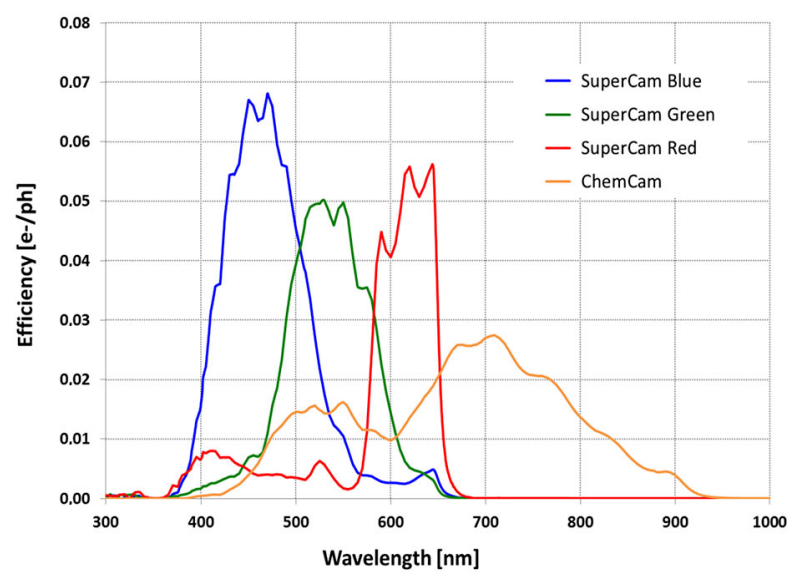

\subsubsection{Infrared Spectrometer}

The IR Spectrometer (IRS) opens a new wavelength range from 1.3 to $2.6 \mu \mathrm{m}$ for exploration from the surface of Mars. It is designed as a point spectrometer to characterize the same targets as LIBS and TRR/L from 1.5 to $7 \mathrm{~m}$. The IRS benefits from the unique capability of LIBS to remove dust. Beyond $7 \mathrm{~m}$, the IRS can be used all the way to infinity. Since the telescope étendue is constant, the amount of light reaching the detector at long distance is the same as at short distance, the FOV covering a larger surface. This description of the IRS is intentionally short; the readers should refer to a future dedicated publication by LESIA for details. The SuperCam IR spectrometer is inherited from the SPICAM and SPICAV instruments, which are instruments on Mars Express and Venus Express (Montmessin et al. 2017).

The Acousto-Optic Tunable Filter (AOTF) provided by Gooch and Housego (Iliminster, England) is the heart of the spectrometer and performs the wavelength selection (Fig. 29). A radio-frequency signal drives a transducer, which is glued to the side of the AOTF. For each frequency of the piezo between 33 and $68 \mathrm{MHz}$, a narrow band between 2.6 and $1.3 \mu \mathrm{m}$ is transmitted by the crystal, and deflected by $\pm 6.5^{\circ}$ ( 2 polarizations or diffracted orders). 

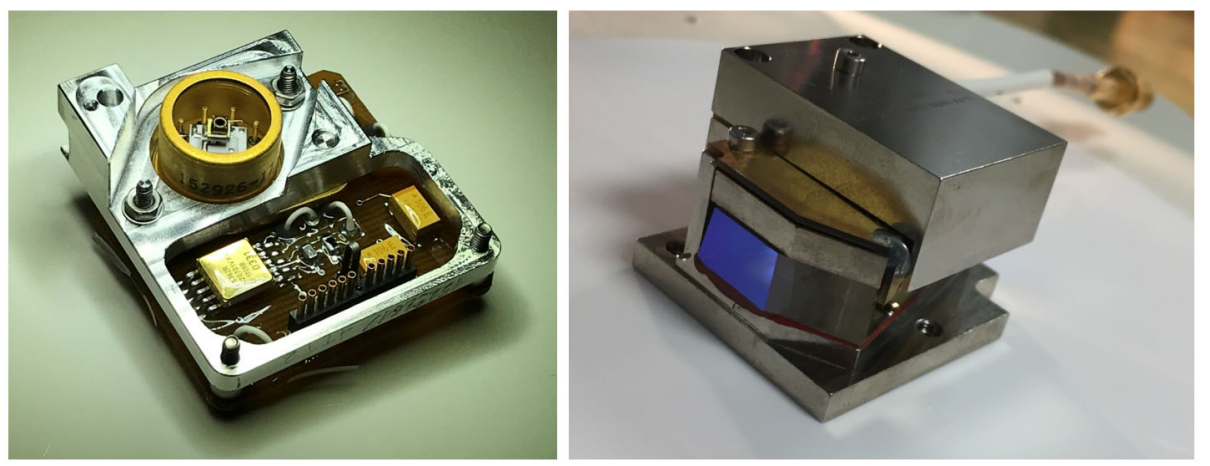

Fig. 29 IRS critical hardware: (Left) the photodiode with TEC, and its front-end-electronics. (Right) the AOTF - blue crystal - with its transducer on the right and on top, under the cover, a matching board with capacitors and coils to maximize the transfer of the RF power to the piezo. (Credit: LESIA)

Fig. 30 The IRS spectral resolution (photodiode 1) measured for three temperatures of the spectrometer. The requirement is $32 \mathrm{~cm}^{-1} \mathrm{FWHM}$, which corresponds to $12 \mathrm{~nm}$ at $1.95 \mu \mathrm{m}$. The actual resolution is better than $25 \mathrm{~cm}^{-1}$, i.e., smaller than $9.5 \mathrm{~nm}$

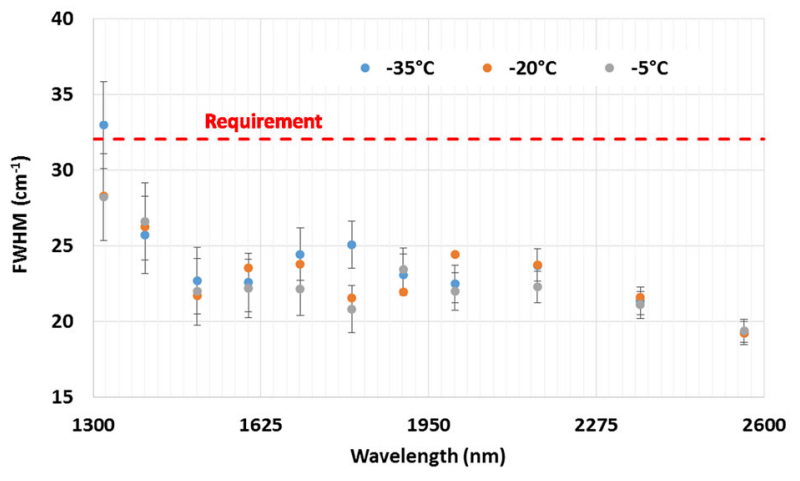

One polarization is registered on a $1 \mathrm{~mm}$ diameter Mercury Cadmium Telluride (MCT) photodiode, the other one ends up on another photodiode for redundancy (Fig. 31). These commercial off-the-shelf (COTS) photodiodes, which are provided by Judson Teledyne (Montgomeryville, PA) are packaged with a triple-stage thermoelectric cooler (TEC) to lower their temperature and thus the dark current. The TEC can lower the photodiode temperature by $80{ }^{\circ} \mathrm{C}$ below that of the spectrometer (verified in the laboratory) although a smaller delta is usually sufficient. Cooling is typically achieved within 2 minutes. These COTS photodiodes were qualified through a lot-acceptance test program.

Both AOTF and photodiodes are assembled in the IRbox (Figs. 31, 32), which is mounted on the side of the OBOX. The thermal coupling is efficient enough to dump heat through the whole structure. A periscope links the IRbox to the main telescope. The transmission from the telescope aperture to the IRS entrance is $\sim 30 \%$. The entrance of the IRbox is a pinhole (400 $\mu \mathrm{m}$ in diameter, 0.18 numerical aperture), which defines the IRS FOV: $1.15 \mathrm{mrad}$ (see Fig. 51 right). It is slightly larger than that of LIBS and TRR/L $(=0.75 \mathrm{mrad})$, but smaller than the dust-free area cleared by LIBS. The optical axis of the IRS is offset by $0.3 \mathrm{mrad}$ with regards to LIBS, and stable over the operating temperature range.

One difficulty of the optical design is to trap within the IRbox the zero-order illumination, which carries $1500 \times$ more flux than the diffracted orders. IR-dark coatings lower the total integrated scatter below $3.5 \%$. Thanks to staggered walls and coatings, less than $0.12 \%$ of 


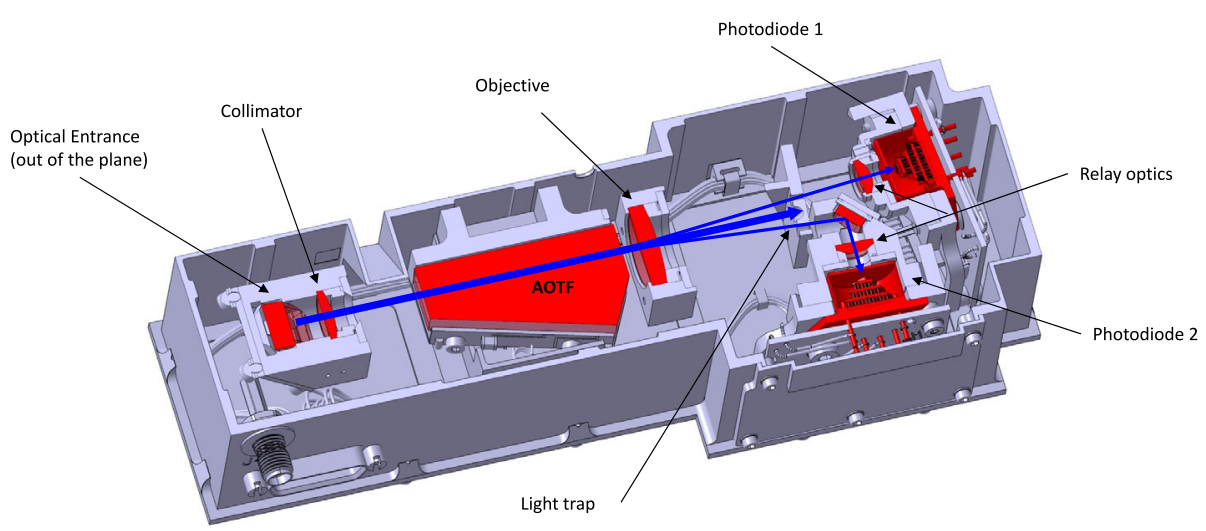

Fig. 31 IR spectrometer cross section. Optical pieces are in red. Light follows the straight blue path, mostly to the light trap. Depending on the piezo frequency, one wavelength is diverted to photodiode 1, and the other order to photodiode 2 (backup)

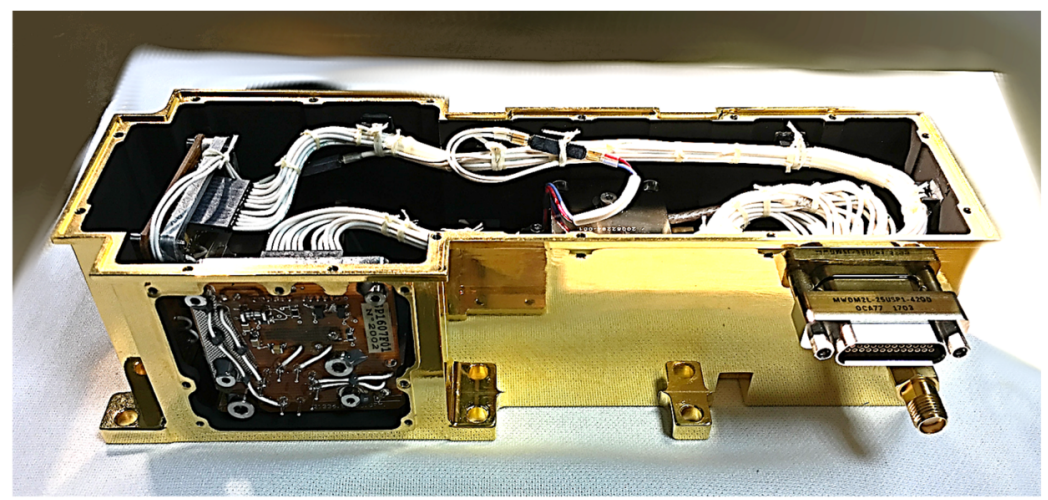

Fig. 32 The IR Spectrometer. The cover is open. On the left, the two photodiodes (the FEE of the photodiode 2 is visible). To the right, the main connector to both FEE and temperature sensor, and below, the RF shielded connector to the AOTF. The total length is $170 \mathrm{~cm}$ and the mass is $402 \mathrm{~g}$. (Credit: LESIA)

the flux exits the light trap. The light trap also baffles the photodiodes, which can only see black-coated surfaces.

Regarding electronics, front-end drivers to amplify the photodiode current and for the TEC are at the rear of each photodiode, while the generation of the RF signal and service functions are on a separate IR board, located in the EBOX. The challenge for the IR board is to maintain the readout noise level below $0.1 \mathrm{pA}$. The AOTF crystal length itself gives the IRS resolution of $<32 \mathrm{~cm}^{-1}$ FWHM, i.e. a resolution $<12.1 \mathrm{~nm} @ 1.95 \mu \mathrm{m}$ (Fig. 30). 128 spectels (channels) are necessary to cover the full spectral range, but over-sampling with $15 \mathrm{~cm}^{-1}$ steps is also possible. 16 registration tables exist in the instrument, consisting of different arrangements of spectels to be read: only 3 spectels for rapid scan mode, 86 spectels to focus on the major absorption bands of interest, 128 spectels evenly spaced in wavelength or wavenumber, and 256 spectels. Even one single spectel can be commanded.

To acquire an IR spectrum, the following parameters are set: the photodiode integration time, $T_{\text {int }}$, which is the same for all the spectels; the number of spectels and their spectral dis- 
Fig. 33 Signal-to-noise simulations for the IRS, as a function of local time (HH:MM), for a thermal environment close to Jezero crater and a start of the instrument at 13:00. This is a worst case hot (see text). Four SNR simulations are shown, as the instrument is warming up, for 86 spectels acquired in $80 \mathrm{~s}$ (signal and dark). The requirement is $\mathrm{SNR}=56$

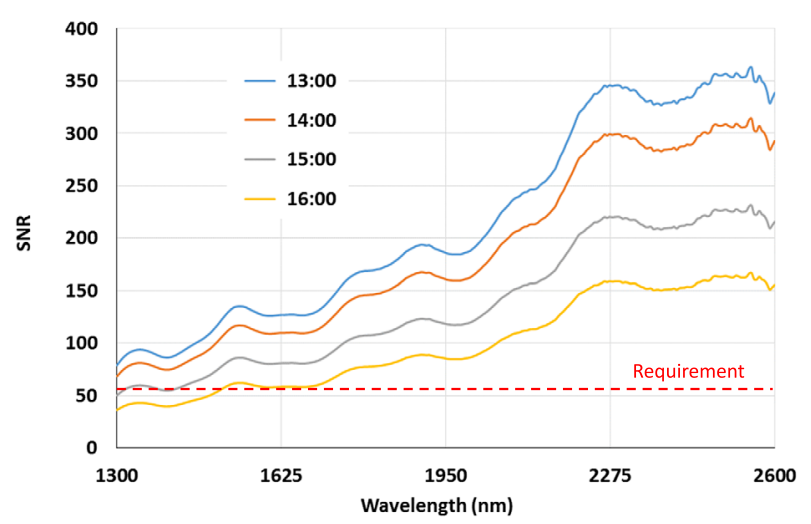

tribution (one of 16 registration tables); and the number of accumulations $\left(\mathrm{N}_{\mathrm{acc}}\right)$ per spectel, which can be different spectel to spectel but preset in a $\mathrm{N}_{\text {acc }}$ table. An auto-exposure mode can automatically set the exposure to avoid saturation, based on dark current. A fixed step of $136.33 \mathrm{kHz}$ in RF bandwidth from 33 to $68 \mathrm{MHz}$ is necessary to address each spectel of the registration tables. Based on these parameters, the photodiode signal is acquired with RF power on (signal) and off (dark). The sequence may be repeated $\mathrm{N}_{\text {acc }}$ times to increase $\mathrm{SNR}$. The $\mathrm{N}_{\mathrm{acc}}$ acquisitions for both dark and signal are summed by the IRS itself.

To track the performance during the instrument development, a detailed radiometric model was developed to assess the SNR obtained with various spectrometer temperatures, instrument parameters, and local times of observation. Analyses, validated by tests, demonstrated that $\mathrm{SNR}>70$ is obtained in the $\left[-40{ }^{\circ} \mathrm{C} ;-5^{\circ} \mathrm{C}\right]$ temperature range expected for the instrument at Jezero crater (Fig. 33). The lone exception is for acquisitions taken late in the day, and even then the SNR criterion is missed only at low wavelengths in worst case hot situations (Royer et al. 2020). For these simulations, the total integration time is $<80 \mathrm{~s}$; it can be increased to boost the SNR; for example, SNR $>400$ can be reached for better quality data.

A comprehensive IR calibration campaign of the SuperCam flight unit was performed before delivering the instrument to JPL. See Royer et al. (2020) for a detailed description. As a summary, the absolute response is accurately estimated using a statistical algorithm. A worst-case approach, based on the quadratic sum of the linearity in charge, the linearity in flux, and the absolute blackbody accuracy yields an absolute accuracy $<15 \%$ (20\% was the requirement). For the spectral response, the error is a combination of the SNR and the high-frequency behavior of the instrument. We find $0.8 \%$ maximum relative error over the whole spectral and thermal ranges (1\% was the requirement).

Finally, the rover carries two Lambertian calibration targets dedicated to the IRS: Aluwhite (IR) and Black paint (IR); other targets may be used as well. The team plans to acquire signals from these targets under different Sun elevations. See Manrique et al. (this journal) for details.

\subsubsection{Focus Mechanism}

To focus on targets from $1.56 \mathrm{~m}$ to infinity, the secondary mirror is mounted on a translation stage. The translation is implemented by a 2-phase stepper motor ZSS-25-200-0.6 HV from Phytron, Germany (Fig. 34). The motor is coupled to a screw/nut assembly that connects to a leadscrew on the stage side. The screw/nut system is not preloaded in order to increase 
Fig. 34 Supercam focus mechanism. (Credit: CNES/Alexandre Ollier, 2019)

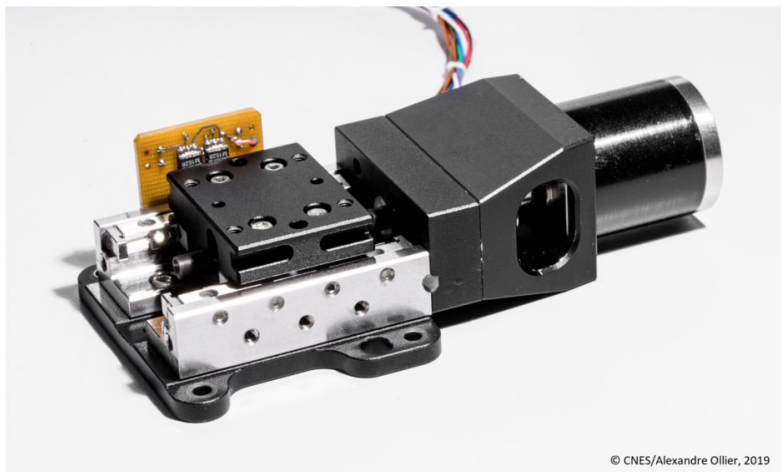

Fig. 35 The translation stage motorization margins: minimum current for the complete displacement divided by nominal current ( $0.6 \mathrm{~A}$, blue) and boost current (0.8 A, red). Below $-30{ }^{\circ} \mathrm{C}$, the speed is slowed down from 150 steps/s to 100 steps/s. The "no motion" line is drawn with no margins. The ECSS recommend a $\times 3$ margin

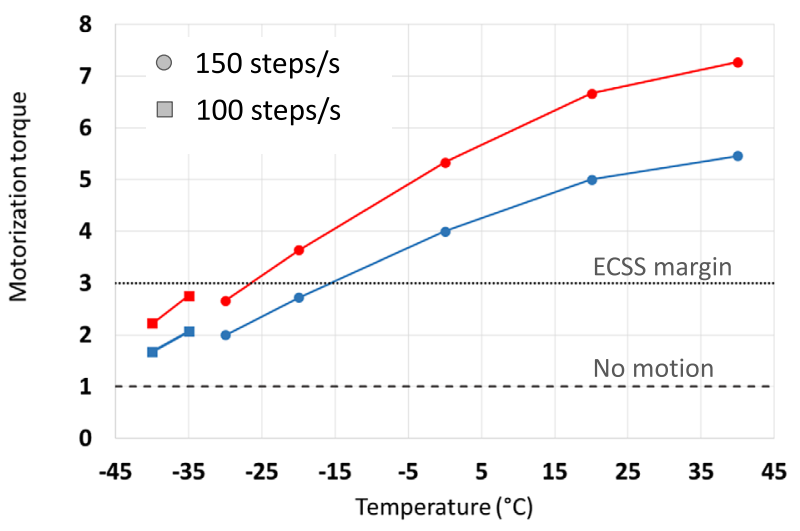

the mechanism lifetime; longitudinal backlash is corrected within the autofocus procedure itself. The mobile plate displacement for one motor step is $1.25 \mu \mathrm{m}$. Stainless steel rails guide the mobile plate. A $45^{\circ}$ roller and cage assembly increases the lateral and vertical stiffness. This translation stage is built to print from ChemCam, and was delivered by PI miCos, Germany, as a commercial product. See Maurice et al. (2012, Sect. 3.2.3) for details.

The motor is driven at $0.6 \mathrm{~A}$, providing a holding torque at $13 \mathrm{mN}$.m. When the windings are not energized, the detente torque is $2 \mathrm{mN}$.m; this is enough to hold the assembly in place with no latching mechanism. For safety and end-of-life margins, we have implemented a boost mode at $0.8 \mathrm{~A}$, which corresponds to a holding torque of $17.3 \mathrm{mN}$.m. The nominal driving frequency is 150 steps/s. However, to meet European Cooperation for Space Standardization (ECSS) standards at end of life (7,000 autofocus cycles) or to come close to them (a factor $\times 3$ is required) a slower speed of $100 \mathrm{steps} / \mathrm{s}$ is used below $-30{ }^{\circ} \mathrm{C}$ (Fig. 35).

Limit switches are the only difference from the ChemCam's focus mechanism. There is only one on ChemCam, a differential magneto-resistive sensor coupled to a Permalloy pellet that is glued on the movable plate. It can detect the close focus with a $\pm 20 \mu \mathrm{m}$ accuracy. For SuperCam, we decided to use two Hall Effect (Optek OMH3075B) sensors on each end of the translation stage. The sensors are mounted on a PCB that is fixed to the side of the structure, while two magnets from Arnold Magnetic (Recoma 28) are carried by the moving plate. On the motor side, the limit switch is called Infinite Focus (IF), and on the other side, toward the exterior of the telescope, there is the Close Focus (CF) switch. The Hall Effect/magnet pairs are reversed to allow triggering of the sensors by the North poles of 


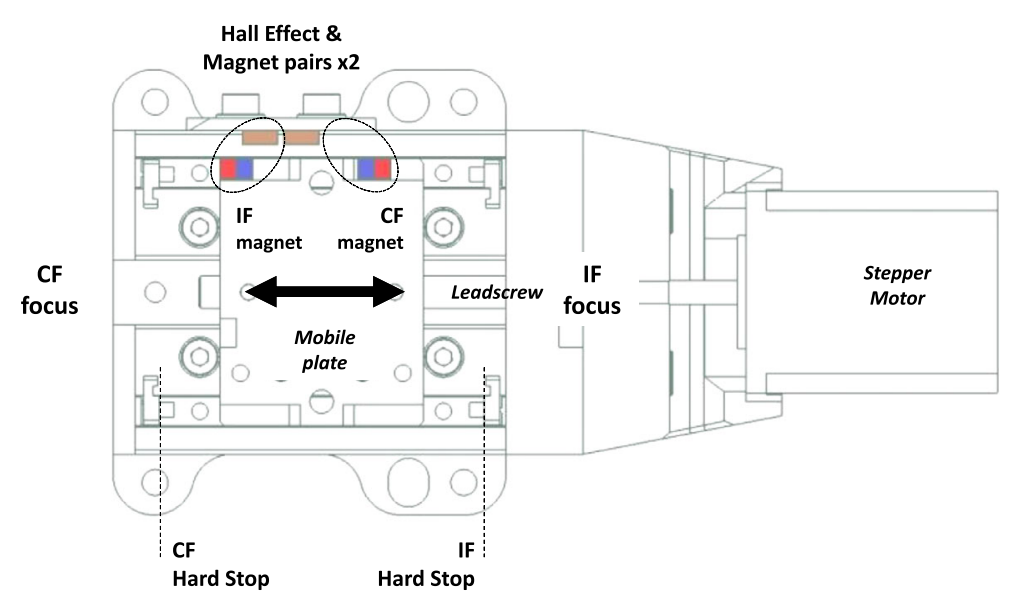

Fig. 36 Translation stage schematics with details on the focus assembly: paired Hall effect sensor and magnets, hard stops, close focus (CF) and Infinite Focus (IF) directions

the magnets (Fig. 36). Beyond each sensor, there are hard stops that are not supposed to be reached in nominal operations. On the flight unit, these two hard stops are $15.42 \mathrm{~mm}$ apart, thus separated by 12,336 steps.

Each limit-switch sensor is excited by the magnet to which it is paired (Fig. 36). The output voltage of the sensor depends on the magnetic intensity seen by its Hall Effect loop: if the intensity increases above a given positive threshold, Vout $=$ Vin $=3.3 \mathrm{~V}$ the proximity electronics turns the sensor status $\mathrm{ON}$; if the intensity is below a given negative threshold, Vout $=0 \mathrm{~V}$, the sensor status turns OFF. Each sensor has a unique set of thresholds to latch $(\mathrm{ON})$ or delatch (OFF). After the magnet comes close to the sensor to latch it, it needs to move away from the sensor in the opposite direction to delatch. Hence there is an inherent hysteresis associated with this design. At $20{ }^{\circ} \mathrm{C}$, it is $\sim 2,200$ steps $(1.76 \mathrm{~mm})$ that must be taken into account for the design of the focus algorithm. Several reproducibility tests yield a $\pm 10 \mu \mathrm{m}$ accuracy for latching/delatching. The sensitivity of Hall Effect sensors varies with temperature. Thus, latch positions and hysteresis have been measured with temperature (Fig. 37); the $\mathrm{CF}$ position varies by $\sim 100$ steps over $\left[-30{ }^{\circ} \mathrm{C} ;+10^{\circ} \mathrm{C}\right]$, while the IF varies by $\sim 150$ steps. The hysteresis changes roughly by the same number of steps.

The optical requirement to point at targets as close as $1.56 \mathrm{~m}$, possibly down to $1.1 \mathrm{~m}$, and up to infinity is met by moving the secondary mirror by $12.5 \mathrm{~mm}$, which corresponds to 10,000 steps between the two limit switches. Figure 37 describes the situation at different temperatures, accounting for the drift of the latch positions described above. The sensors appear to be well balanced. The available stroke is indeed more than 10,000 steps at all temperatures, and there are similar margins with regard to the hard stops on both ends, about $6 \%$ of the steps. This is very large when one considers the 8-step accuracy on the limit switches to stop the stroke.

The position and stability of the translation stage inside the telescope is critical: the secondary mirror needs to remain aligned within $50 \mu \mathrm{m}$ of the primary mirror axis. The stroke must be at least $12.5 \mathrm{~mm}$ to cover the range of targets to infinity. During thermal testing, focus on different targets and at infinity was done to derive a relationship between motor steps and the true distance to targets. The following relationship is used: $y=(a x+b) /(x+d)$, with $y$ in $[\mathrm{m}]$ and $x$ in [steps]. $x$ is referenced to the CF limit switch, since it is the only 


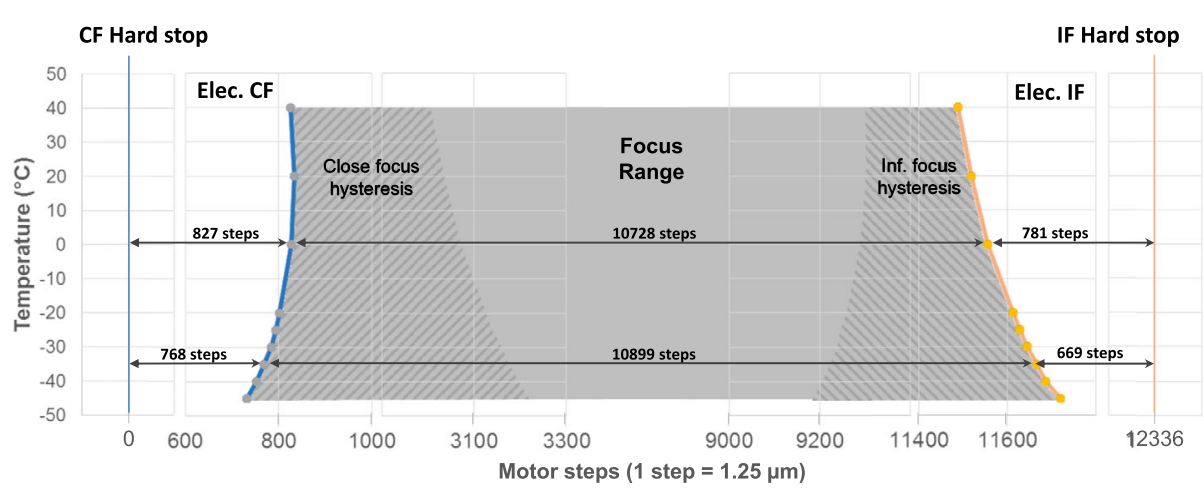

Fig. 37 The optical requirement for the focus range is $12.5 \mathrm{~mm}(10,000$ steps). The maximum range for this stage is $15.42 \mathrm{~mm}(12,336$ steps). The horizontal axis is broken into small sections to highlight the most relevant information

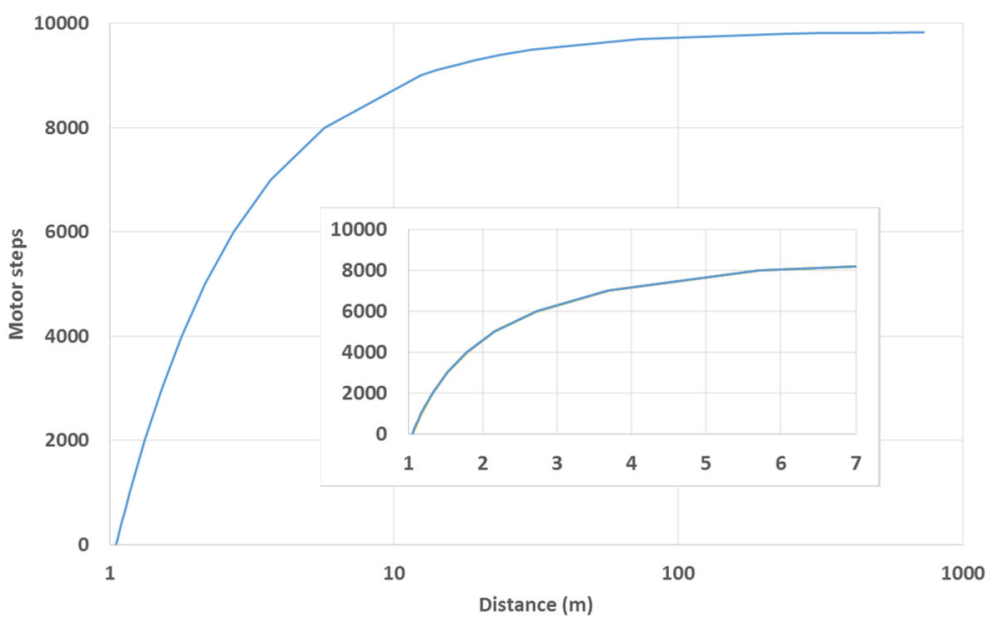

Fig. 38 Motor-steps to distance law, from the $\mathrm{CF}$ limit switch at $-30{ }^{\circ} \mathrm{C}$. The inset is for close distances between 1 and $7 \mathrm{~m}$

known reference onboard. Several fitting methods were tested (Fig. 38). We find:

$$
\begin{gathered}
d=-9839+0.27 \mathrm{~T}+0.026 \mathrm{~T}^{2}+0.00077 \mathrm{~T}^{3}-0.000021 \mathrm{~T}^{4} \\
a=-0.010621+0.000261 \mathrm{~T} \\
b=-10401.7-1.11 \mathrm{~T}
\end{gathered}
$$

These are conversion equations that are coded onboard, where $\mathrm{T}$ is the telescope temperature in ${ }^{\circ} \mathrm{C}$. Below $7 \mathrm{~m}$, the RMS is 5.9 steps, on the order of the latching accuracy. The optical infinity is at $\sim 9860$ steps from the CF hard stop (RMS $=1.6$ steps) at cold; the software can accommodate a second conversion law that will be calibrated in flight for long distances. The closest distance the telescope can focus in principle is for $x=1$ step, which corresponds to $1.05 \mathrm{~m}$ at $-30{ }^{\circ} \mathrm{C}$, closer than the requirement $(1.56 \mathrm{~m})$. 

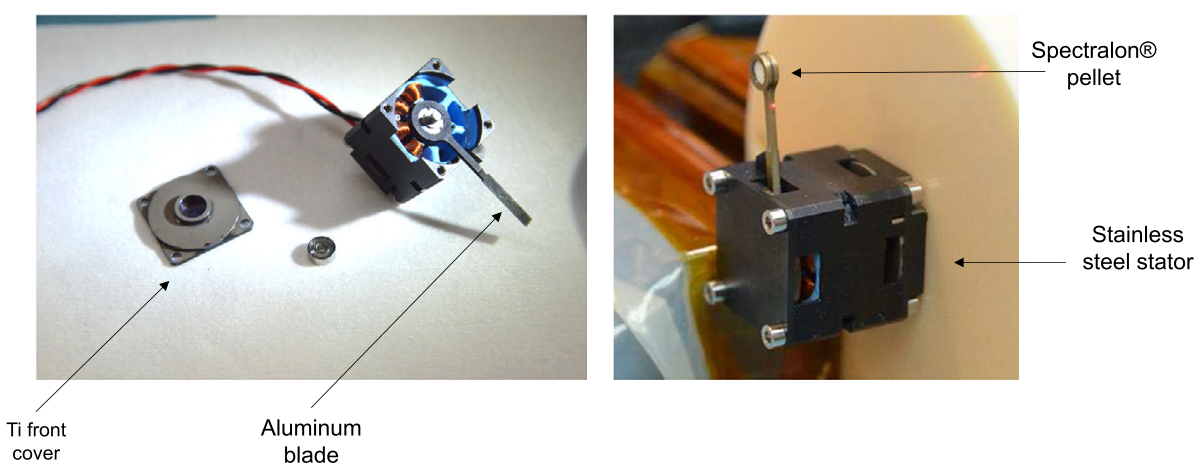

Fig. 39 Shutter mechanism to block the red beam when doing TRR/L. On the left, the motor is open; there is no Spectralon pellet on this blade. To the right, the final configuration of the shutter

\subsubsection{Laser-Beam Shutter}

The generation of the green laser beam produces a significant amount of red residual light beam that needs to be blocked to avoid any LIBS spark on target during TRR observations. To this end, the rotor of an actuator carrying a blade with two stable positions is used: when the power is off, permanent magnets pull the blade out of the red beam path (LIBS mode); when power is on, the blade rotates by $13^{\circ}$ and masks the entrance of the red Galilean (TRR mode). The blade carries a Spectralon ${ }^{\circledR}$ coated surface to scatter the red light (Fig. 39).

This shutter is a spare part from Venus Express/VIRTIS and Rosetta/VIRTIS programs. It has benefited from a thorough re-qualification program that includes a complete stripping of the parts, a change of ball-bearings and blade, and testing to Mars 2020 requirements and lifetime. In the end, the performance is insensitive to temperature; we observed no degradation of performance during the lifetime tests. Under normal circumstances, the shutter is activated for a maximum of $100 \mathrm{~s}$, during which it will warm up by $15^{\circ} \mathrm{C}$, then a rest period is required. This shutter is qualified for 7,000 actuations.

\subsubsection{Microphone}

The microphone was integrated late in the development of the Mast Unit, recognizing that there is an interest in listening to the shock wave produced by the expansion of a LIBS plasma (Chide et al. 2019, 2020b). The microphone can also record wind sounds to contribute to basic atmospheric science, and rover noises, which will very likely help for engineering purposes. These sounds include those of the wheels or the drill. The microphone had to be robust, small in mass and size, and resistant to extreme temperatures. It was agreed with JPL that the microphone would have been descoped at any time if it had become a threat to other investigations. Fortunately, it survived the many challenges inherent to a space project.

In line with numerical models of sound propagation on Mars (Williams 2001; Bass and Chambers 2001), SuperCam's microphone can record pressure waves from $100 \mathrm{~Hz}$ to $10 \mathrm{kHz}$, with sampling rates at $25 \mathrm{kHz}$ or $100 \mathrm{kHz}$. The sensor is a COTS electret, EK23132, the lowest noise microphone manufactured by Knowles (USA). The same model has already flown twice to Mars, on the Polar Lander in 1999 and the Phoenix in 2008. Its sensitivity is $29.6 \mathrm{mV} / \mathrm{Pa}$ at $1 \mathrm{kHz}$ without any stage of amplification and its dimensions are $5.6 \mathrm{~mm} \times 3 \mathrm{~mm}$. Screening and lot qualification were performed on 100 parts. The qualification path included detailed component analyses, and radiation sensitivity evaluation, with 
Fig. 40 Microphone parts. The microphone itself is mounted on the RWEB window bracket. Its front-end-electronics is mounted on the OBOX

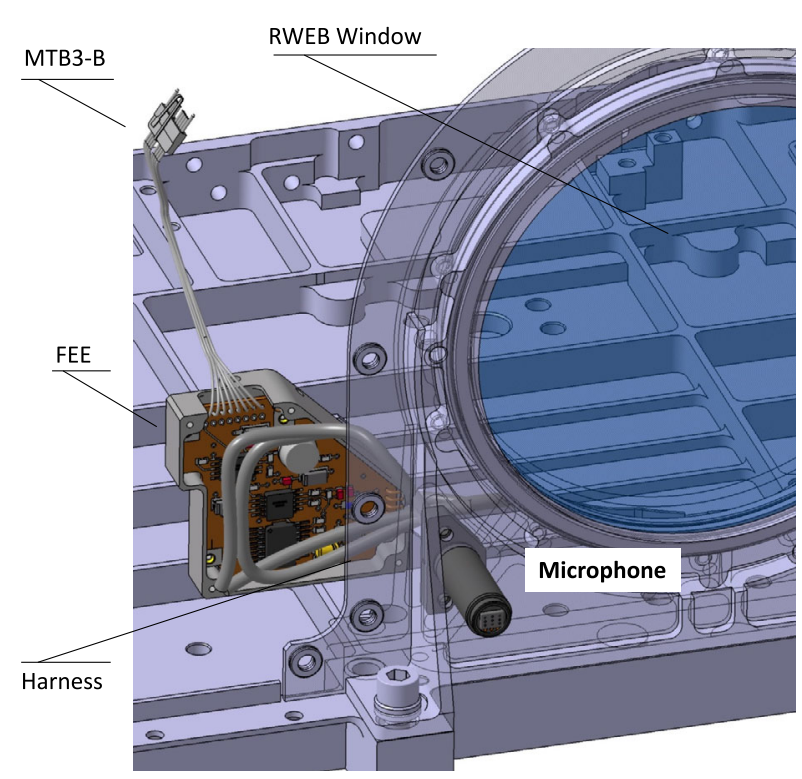

Fig. 41 SuperCam microphone

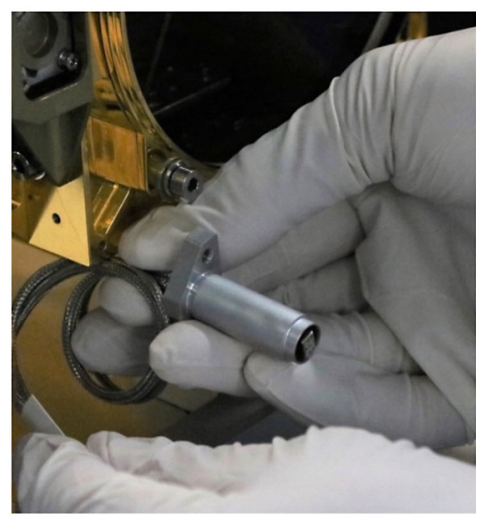

increasing ionizing doses up to $1750 \mathrm{rad}(\mathrm{Si})$. The result is a loss of $10 \mathrm{~dB}$ for the full mission (Radiation Design Factor of 2). We can expect the SNR performance to decrease slowly with time once on Mars. This loss is acceptable for the nominal mission.

The microphone is located outside the Remote Warm Electronics Box (RWEB), at the tip of a $3 \mathrm{~cm}$ aluminum post (Fig. 40). Hence, the echo from the RWEB itself arrives between $222 \mu$ s and $277 \mu$ s after the direct signal. A temperature sensor is also potted inside the microphone stand. The microphone is the coldest point of the instrument and is qualified to $-135^{\circ} \mathrm{C}$.

A shielded cable connects the microphone and temperature sensors to a Front-EndElectronics (FEE) card inside the OBOX (Fig. 41). The harness consists of five Manganin ${ }^{\circledR}$ wires to limit the thermal leak. When the electret is at $-120{ }^{\circ} \mathrm{C}$ and the $\mathrm{OBOX}$ at $-35^{\circ} \mathrm{C}$, the power drawn from the survival heaters by the microphone is only $\sim 30 \mathrm{~mW}$, four times smaller than for standard copper wires of the same diameter. 
The role of the FEE is to amplify and filter the analog signal and to collect temperature data. A first stage amplifies the signal by a factor of 15 , a second stage by a controllable gain, $\times 2, \times 4, \times 16$, and $\times 64$. The digitalization of the signal is performed by a fast 12-bit analogto-digital converter (ADC) on the instrument DPU board. To avoid failure propagation, two short-circuit protections are implemented for the microphone and the operational amplifiers.

In the end, we have noted that the microphone is sensitive to electromagnetic noise from the rest of the experiment, but fortunately not from the rover equipment as could be checked during ATLO tests at JPL. The perturbations come from the laser stack heater at $\sim 76 \mathrm{~Hz}$ and its harmonics (the non-laser, standalone microphone mode is not affected since the laser thermal control is inactive). Since the laser-induced signal is repeated several times (as many times as the number of laser shots), an average waveform can be built from the stacking of the various signals, which have been filtered in the time and frequency domains. The correlation of each pulse with the average signature allows us to retrieve a signal cleaned from the perturbation after several iterations.

Each MIC data product is required to be $\leq 8 \mathrm{MB}$, the volume of an uncompressed RMI image. The microphone has three recording modes: i) the standalone mode for natural and artificial sounds, up to $2.46 \mathrm{~min}$ at $25 \mathrm{kHz}$, ii) the LIBS continuous mode around a burst of laser shots, up to 120 shots, which at $3 \mathrm{~Hz}$ is equivalent to $40 \mathrm{~s}$ of recording, and iii) the LIBS pulsed mode. In the latter mode, the microphone is synchronized with the pulsed laser frequency. For each laser shot, the recording starts $500 \mu$ s before the laser fires and lasts for $60 \mathrm{~ms}$. The shock-wave direct signal is expected to arrive between $5.5 \mathrm{~ms}$ (for a target at $1.5 \mathrm{~m}$ at $10{ }^{\circ} \mathrm{C}$ ) and $18.5 \mathrm{~ms}$ (for a target at $4 \mathrm{~m}$ at $-90^{\circ} \mathrm{C}$ ) after the laser shot. Both LIBS modes are executed at $100 \mathrm{kHz}$ sampling frequency. At a laser frequency of $3 \mathrm{~Hz}$ for 30 shots, the product size is only 0.32 Mbytes, compared to 2.2 Mbytes in the LIBS continuous mode. An optional down-sampling to $25 \mathrm{kHz}$ is implemented in the Body Unit if needed.

More microphone details can be found in a future dedicated publication by ISAE.

\subsection{Optical Design}

SuperCam Mast Unit is, first and foremost, an optical instrument that is built around an optical bench, also called the Optical Box (OBOX). It contains different sub-assemblies: a Schmidt-Cassegrain telescope, two Galilean beam expanders, a dioptric objective and a Beamsplitter. In the OBOX, the red laser is focused for LIBS, the green laser is collimated for TRR, and light is collected for LIBS, TRR/L, VISIR, and RMI.

\subsubsection{Description}

The complexity of the design stems from the spectral range that needs to be covered (Fig. 42). There are 3 laser beams, at $1064 \mathrm{~nm}$ (red), $532 \mathrm{~nm}$ (green), and $852 \mathrm{~nm}$ (CWL) that exit the Mast Unit. Collected photons from $245 \mathrm{~nm}$ to $853 \mathrm{~nm}$ need to be sent to the Body Unit, via an optical fiber. There are 3 analyses that are performed within the OBOX: processing of the CWL autofocus signal at $852 \mathrm{~nm}$, imaging from 400 to $650 \mathrm{~nm}$, and infrared spectroscopy from 1.3 to $2.6 \mu \mathrm{m}$. The optical design to cover this complexity relies on different assemblies:

- The RWEB window is the interface with the Martian environment outside. It is a flat piece of low-OH silica that is mounted on the camera bar that supports the Mast Unit and the RWEB cover, as well as other rover hardware (Fig. 43 and Fig. 44, optical component $\# 0)$. 


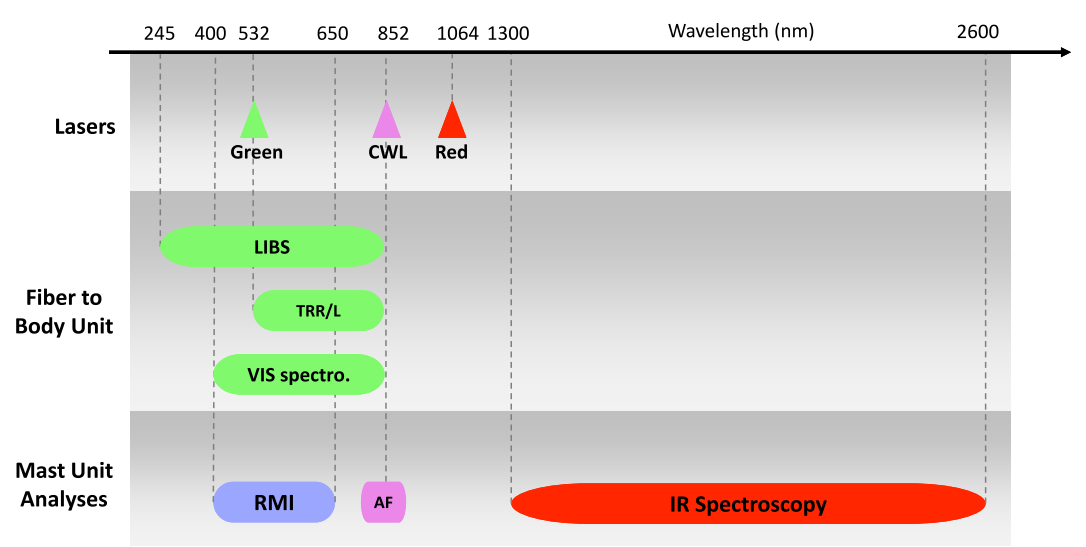

Fig. 42 Overview of spectral bands within the Mast Unit: the lasers (top), the analysis performed in the Body Unit via the mast-to-body fiber (middle), and the analysis performed in the Mast Unit itself

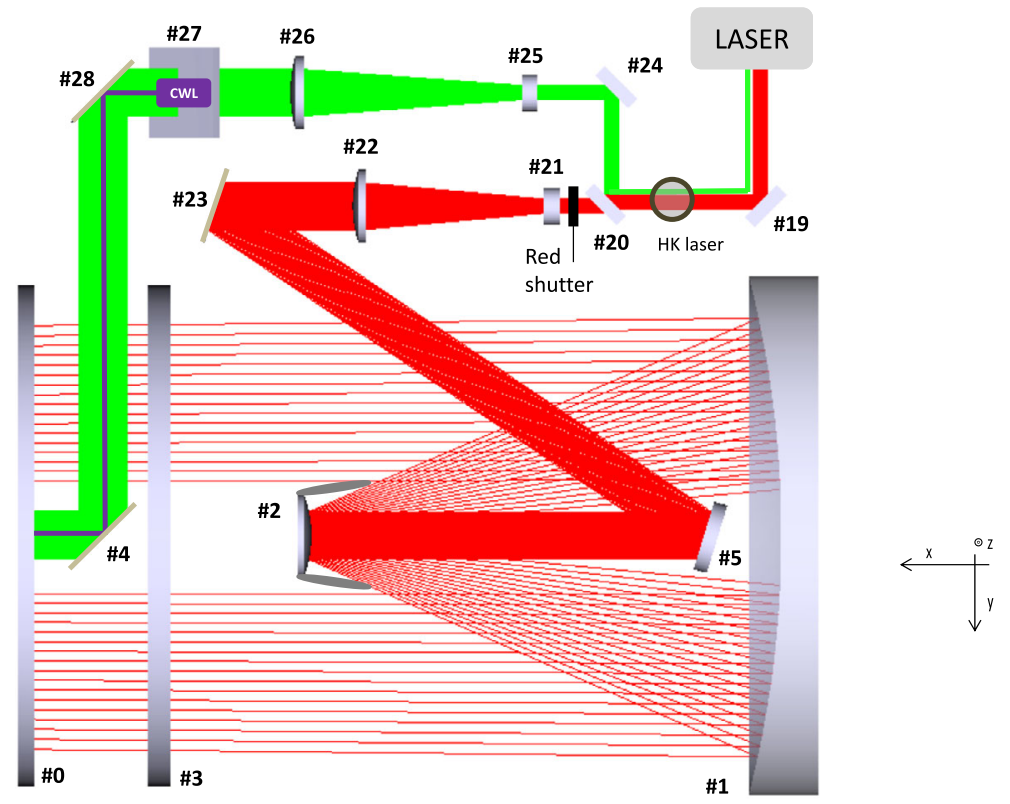

Fig. 43 Optical layout of the Mast-unit Cassegrain and Galilean telescopes. The three laser paths going out of the telescope toward the target are shown: red beam at $1064 \mathrm{~nm}$ (red), green beam at $532 \mathrm{~nm}$ (green), and CWL beam at $852 \mathrm{~nm}$ (purple)

- The Schmidt-Cassegrain telescope is the centerpiece of the OBOX. Its architecture is similar to ChemCam's: a primary mirror (110 mm dia., Fig. 44 \#1), a secondary mirror (16 mm dia., \#2), and a Schmidt plate (max surface sag: $87 \mu \mathrm{m}, \# 3$ ). They all work in concert. The telescope can focus from $\sim 1.1 \mathrm{~m}$ to infinity by changing the position of the secondary mirror using a translation stage (see Sect. 4.3.4). 


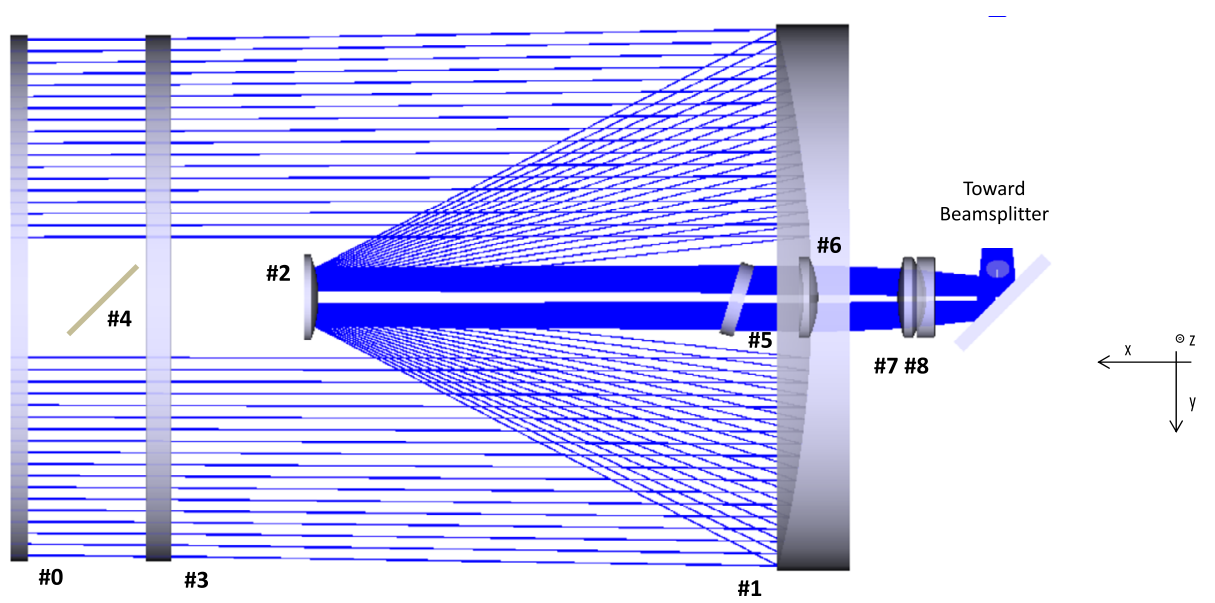

Fig. 44 Optical schematics of the Mast-Unit Cassegrain telescope and objective. Light collected from the target between 245 and $2600 \mathrm{~nm}$ is shown in blue. (The range from 860 to $1300 \mathrm{~nm}$ is rejected by the dichroic \#5)

- The red beam is expanded from $3.3 \mathrm{~mm}$ to $1 \mathrm{~cm}$ by a compact Galilean telescope (optics \#21 and \#22), which is built to print from ChemCam. At the entrance of this Galilean, scattered light is captured to monitor the red laser energy output. A shutter blocks the red beam when TRR is performed (see Sect. 4.3.5); otherwise the red beam is then injected into the Cassegrain telescope. The green beam is also expanded to $1 \mathrm{~cm}$ by a compact Galilean telescope (optics \#25 and \#26), but it remains collimated and is co-aligned with the Cassegrain telescope boresight by a 2-mirrors periscopic system (optics \#28 and \#4). The CWL is injected along the same path between the green Galilean and the 2-mirror periscope. It stays collimated along the telescope optical boresights.

- The 3-lens objective focuses part of the visible light on the imager, and injects the rest of the flux into the Beamsplitter (Fig. 44, optics \#6-8).

- The Beamsplitter redistributes photons to the AF photodiode, the mast-to-body fiber, the IRS, and the RMI (Fig. 45). The path to the IRS is a periscope (optics \#13, \#31-33); the entrance pupil is a $400 \mu \mathrm{m}$ pinhole with 0.18 numerical aperture. Optics on the path of light to the IRS are low-OH. Below $1 \mu \mathrm{m}$ wavelength, the light is injected at $0.15 \mathrm{NA}$ into the fiber (optics \#14 and \#17), whose core is $300 \mu \mathrm{m}$ diameter with 0.22 numerical aperture.

Hence, the OBOX consists of $>30$ optical parts (Table 5), most of which are custom made. The primary mirror is the most distinctive piece of the design (Fig. 43, optics \#1). Largely inherited from ChemCam, the extruded structure is made of aluminum (see next section, last paragraph). The optical surface shape error is $17 \mathrm{~nm} \mathrm{rms}$, and roughness is $\sim 4.5 \mathrm{~nm} \mathrm{rms}$.

Most of the structures carrying these optics are painted black (PNC by MAP Coatings, France) to reduce stray light for the RMI, which has a relatively large field of view $\sim 18.8 \mathrm{mrad}$. There is also a baffle around the secondary mirror. Its outer diameter is $27.5 \mathrm{~mm}$, and its inner diameter circles the secondary mirror. The side effect is a loss of $\sim 1 \%$ of the red beam for LIBS. 


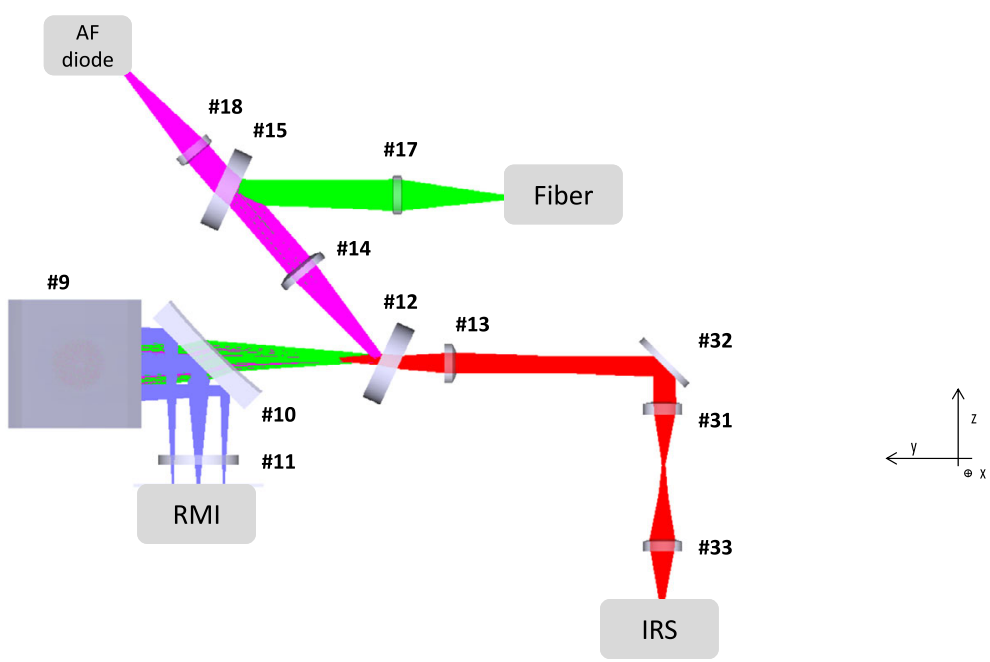

Fig. 45 Optical schematics of the Mast-Unit Beamsplitter. Light coming from the target at $852 \mathrm{~nm}$ is distributed to the AF diode (purple), to the IRS from 1300 to $2600 \mathrm{~nm}$ (red), to the RMI from 400 to $650 \mathrm{~nm}$ (blue), to the mast-to-body fiber from 245 to $852 \mathrm{~nm}$ (green)

\subsubsection{Focusing the Red Laser}

There are 2 key and driving requirements for LIBS: the energy on target needs to be $\geq 12 \mathrm{~mJ}$ at $1064 \mathrm{~nm}$, and the irradiance $\geq 10 \mathrm{MW} / \mathrm{mm}^{2}$ up to $7 \mathrm{~m}$ within a circle that contains $75 \%$ of the deposited energy. The difficulty is to reach these thresholds up to $7 \mathrm{~m}$ and over the $\left[-30{ }^{\circ} \mathrm{C} ;+10^{\circ} \mathrm{C}\right]$ range. The transmission of the $1064 \mathrm{~nm}$ laser beam through the telescope is $\sim 50 \%$ because of the beam's Gaussian distribution and the central obscuration of the Schmidt-Cassegrain telescope. As the laser delivers more than $24 \mathrm{~mJ}$ in red (see Sect. 4.3.1), the energy requirement is met, and the intensity is constant with distance and temperature. Figure 46 presents LIBS Point Spread Functions (PSFs) at $-25^{\circ} \mathrm{C}$ and 3 distances. The first theoretical Airy circle is overlaid:

$$
r=\frac{2 \lambda R\left(M_{2}\right)^{2}}{\pi D}
$$

for its radius, with $R$ the distance to target, $D$ the telescope aperture diameter for the red laser (=90 mm), $M_{2}$ the beam quality. A dedicated high-flux camera was used to record PSFs at those same distances to extract an elliptic mask that contains $75 \%$ of the energy. The mean mask diameters range from 150 to $400 \mu \mathrm{m}$ (Fig. 47, left), somewhat independently of temperature. A linear fit at $-25{ }^{\circ} \mathrm{C}$ from $1.5 \mathrm{~m}$ to $7 \mathrm{~m}$ yields a spot diameter of $100 \mu \mathrm{m}$ to $450 \mu \mathrm{m}$, from which the irradiance can be calculated (Fig. 47, right). With a logarithm fit on data at $-25^{\circ} \mathrm{C}$, the irradiance at $1.5 \mathrm{~m}$ is 10 times the requirement, and meets it at $7 \mathrm{~m}$. Hence, there may be "too much" fluence on the calibration targets at $1.56 \mathrm{~m}$. Two options are available to avoid saturation of the signal: one is to reduce the sensitivity of the detectors (Wiens et al., this journal) and the other is to reduce the laser energy (see Sect. 4.3.1).

As a note, the ChemCam primary mirror has, on top of the Al substrate, a Nickel layer (80-100 $\mu \mathrm{m})$ for polishing, a primer made of Chromium $(30-50 \mu \mathrm{m})$, a reflective Aluminum coating (150-200 nm), and a protective $\mathrm{SiO}_{2}$ layer $(8-10 \mathrm{~nm})$. SuperCam's primary mirror 
Table 5 List of optical components for Figs. 43, 44, and 45

\begin{tabular}{|c|c|c|}
\hline & $\# 0$ & RWEB Window \\
\hline \multirow{3}{*}{ 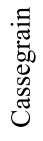 } & $\# 1$ & Primary mirror \\
\hline & $\# 2$ & Secondary mirror \\
\hline & $\# 3$ & Schmidt plate \\
\hline \multirow{6}{*}{ 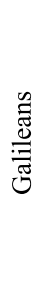 } & $\# 21, \# 22$ & Green Galilean \\
\hline & $\# 4, \# 28$ & Green periscope \\
\hline & $\# 25, \# 26$ & Red Galilean \\
\hline & $\# 20$ & Red/Green dichroic \\
\hline & $\# 27$ & CWL dichroic \\
\hline & $\# 19, \# 24, \# 23$ & Laser splitter \\
\hline \multirow{3}{*}{ 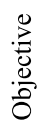 } & $\# 5$ & Laser dichroic \\
\hline & $\# 6, \# 7, \# 8$ & Objective lenses \\
\hline & $\# 9$ & Folding mirror \\
\hline \multirow{7}{*}{ 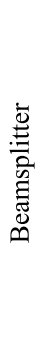 } & $\# 10$ & RMI dichroic \\
\hline & $\# 11$ & IR cutoff filter for RMI \\
\hline & $\# 12$ & IR dichroic \\
\hline & $\# 13, \# 31-33$ & IR periscope \\
\hline & $\# 14, \# 17$ & Fiber collect \\
\hline & $\# 15$ & Notch filter \\
\hline & $\# 14, \# 18$ & AF collect \\
\hline
\end{tabular}

has the same structure and mounting blades as ChemCam's and it was delivered by the same provider, Winlight System (France). However, on SuperCam, to reduce CTE mismatch at cold temperature, the Nickel layer was removed and the polishing occurred directly on the Aluminum substrate. As a result, the performance of the primary mirror was greatly improved at cold $\left(\sim-30{ }^{\circ} \mathrm{C}\right)$ temperature. We have shown that SuperCam's telescope is now diffraction-limited, and the red laser irradiance for LIBS is a factor of $\sim 3$ larger than on ChemCam, due to a tighter beam profile at best focus.

\subsubsection{Collimating the Green Laser}

TRR requires an irradiance between 10 and $30 \mathrm{~kW} / \mathrm{mm}^{2}$, therefore less than $0.3 \%$ of the LIBS threshold to avoid sparking. The first step is to cut off the red beam, activating the shutter (Sect. 4.3.5). The telescope/periscope system transmits $\sim 77 \%$ of the $532 \mathrm{~nm}$ beam with losses that are evenly balanced between optics coatings, and a deliberate vignetting of the beam to avoid collision with the RWEB. Therefore, $\sim 9 \mathrm{~mJ}$ are available outside the Mast Unit (starting from $12 \mathrm{~mJ}$ produced by the laser). Taking into account the magnification factor of the green Galilean, the spot size is $8.4 \mathrm{~mm} \times 9.3 \mathrm{~mm}$ when the beam exits the telescope as a collimated beam. The power density is indeed $\sim 30 \mathrm{~kW} / \mathrm{mm}^{2}$. The green laser is slightly diverging at cold $\left(\sim 1.1 \mathrm{mrad}\right.$ at $\left.-30{ }^{\circ} \mathrm{C}\right)$, and this divergence is divided by a factor of 2.9 by the green Galilean expander. 

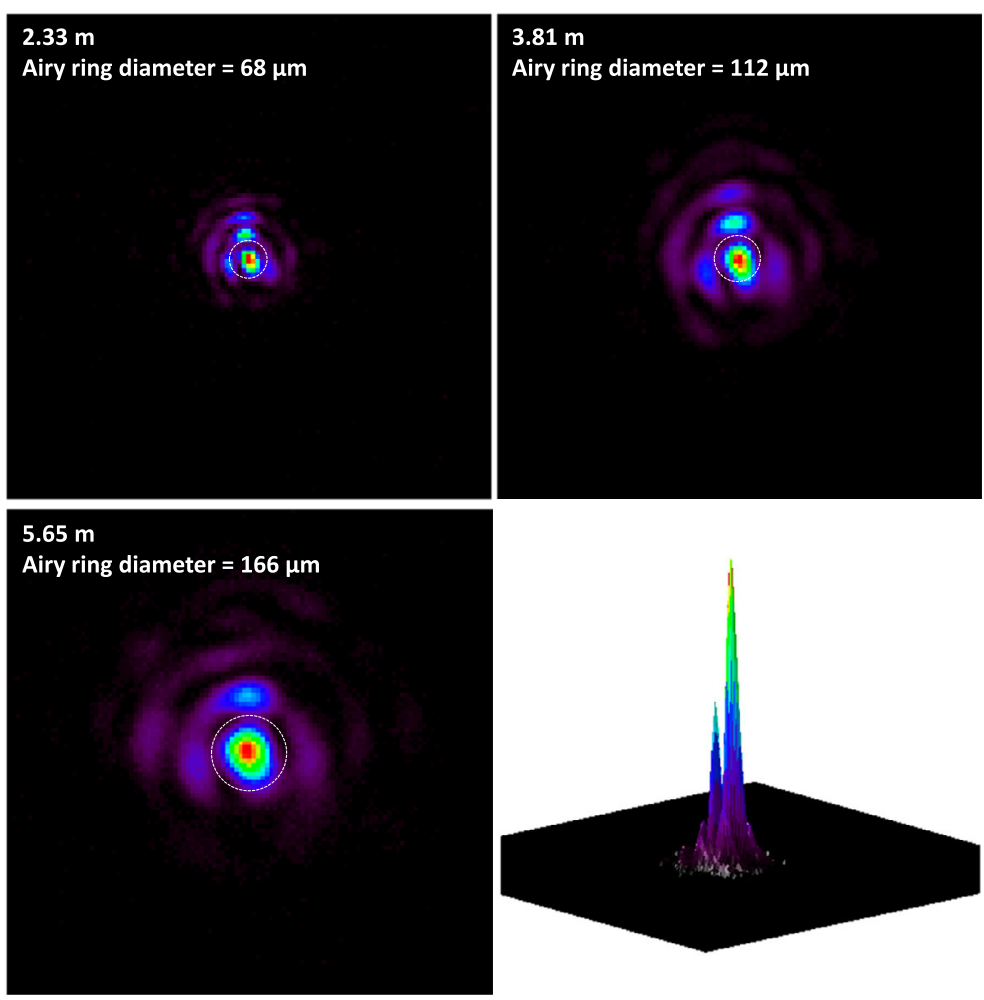

Fig. 46 LIBS point spread function at different distances and $\mathrm{T}=-25^{\circ} \mathrm{C}$. The theoretical 1 st Airy ring is shown. Bottom right, a 3D representation of the laser energy distribution within the LIBS spot, with the same but arbitrary color scale
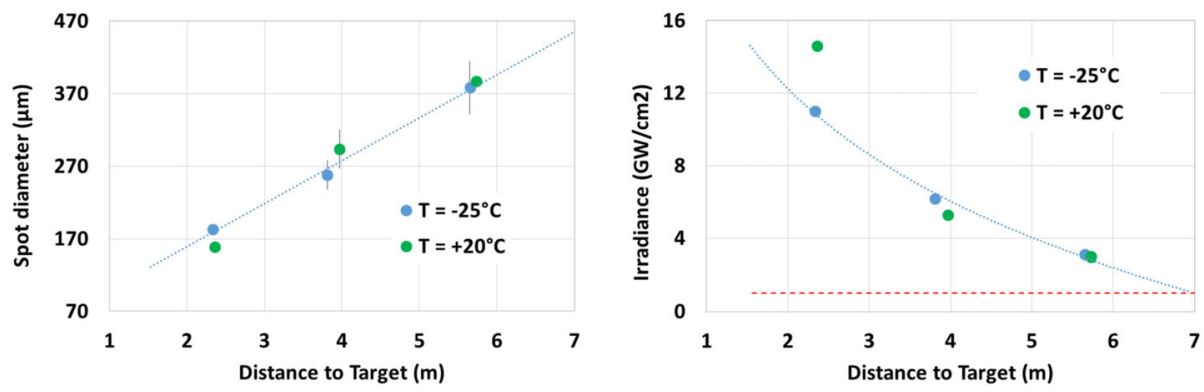

Fig. 47 (Left) Mean diameter of the LIBS mask that contains 75\% of the energy, at three distances and two temperatures. The dashed line is a linear fit at $-25^{\circ} \mathrm{C}$. (Right) Calculated irradiance. The dashed line at $-25^{\circ} \mathrm{C}$ is a logarithm fit. The red line is the requirement

\subsubsection{Light Collection}

For light collection, the transmission is dominated by several custom-made dichroics along the light path. i) The multi-layer laser dichroic filter (optic \#5, Figs. 43, 44) is inherited from ChemCam but with a low-OH substrate and still provided by Cilas (France). It reflects the 
red beam and transmits all other wavelengths, including over the 1.3-2.6 $\mu \mathrm{m}$ range with $\geq 75 \%$ efficiency. It operates at a $17^{\circ}$ incident angle; it has a rebound at $355 \mathrm{~nm}$, which explains the gap between the UV $(\max 340 \mathrm{~nm})$ and VIO $(\min 385 \mathrm{~nm})$ spectrometers in the Body Unit (Wiens et al., this journal). See Maurice et al. (2012, Sect. 3.3.2). ii) For TRR/L, Rayleigh-scattered light at $532 \mathrm{~nm}$ that is reflected by the target needs to be cut off. In the Body Unit, a long pass edge filter provided by Semrock, USA, blocks $99.99 \%$ of this light (Wiens et al., this journal). However, laser light that is Rayleigh scattered from the target can excite a Raman mode in the silica of the mast-to-body optical fiber. To minimize this, a Notch filter provided by Materion, USA, was developed especially for SuperCam and installed in the Mast Unit (optic \#15, Fig. 45). It reflects < 4\% between 531 and $533 \mathrm{~nm}$, and it transmits $>90 \%$ elsewhere. The residual green light that passes through the $6 \mathrm{~m}$ long fiber still generates a small amount of Raman signature, which will have to be removed in the data. iii) The RMI dichroic (optic \#10, Fig. 45) reflects $~ 25 \%$ over $400-860 \mathrm{~nm}$ to the RMI. It has a novel concave rear face to avoid a ghost image that exists on ChemCam.

As a result, Fig. 48 shows the optical transmission to all of the different outputs of the beamsplitter, including the efficiency of the coupling inside the fiber $(90 \%)$ or the IRS pinhole $(92 \%)$, as well as the IR cut-off filter in front of the RMI. For the mast-to-body fiber, transmission to the UV spectrometer is between $16.5 \%$ and $30 \%$, around $30 \%$ for the VIO spectrometer, from $35 \%$ down to $20 \%$ for the transmission spectrometer. This situation is similar to ChemCam, except for the deep cut at $532 \mathrm{~nm}$ created by the Notch filter in the Mast Unit. Altogether, $10 \%$ of the photons go to the RMI; which is sufficient here, since Mars daylight is very bright for this imager (see Sect. 4.3.2). Between 35\% and $40 \%$ of the light goes to the IRS. The design meets its specifications everywhere.

\subsubsection{Optical Axis Alignment}

All techniques (LIBS, TRR/L, VIS, IR, and RMI) need to analyze the same spot on Mars. To that end, all optical axes must be co-aligned with the telescope boresight, which is given by the projection of the fiber on the targets.

For LIBS, the laser is mounted on a special bracket to reduce the intrinsic tilt and decenter of its beams (see Sect. 4.3.1). The remaining tilts are reduced by the magnification factor of the red Galilean and Cassegrain telescope. Thus, the red beam exits the Mast Unit along the telescope boresight. By construction, the optical fiber is along the same axis, and the measurement of their final separation is less than $0.2 \mathrm{mrad}$ over the $\left[-30{ }^{\circ} \mathrm{C} ;+10{ }^{\circ} \mathrm{C}\right]$ operational range. The objective was to make sure that the LIBS spot is well within the fiber FOV, which is $\pm 0.37 \mathrm{mrad}$ wide.

For TRR/L the situation is more complex, since the green beam bypasses the Cassegrain telescope and takes a different route via the green periscope formed by the mirrors \#28 and \#4 (Fig. 43). The former mirror is stable with temperature, while the Schmidt plate motion, whose mounting is not kinematic, holds the second mirror. Thermal environments induce small rotations of the Schmidt plate $\left(\sim 0.6 \mathrm{mrad}\right.$ over $\left.40^{\circ} \mathrm{C}\right)$ that create a tilt of the green laser beam with respect to the telescope boresight along the vertical direction ( $\mathrm{Z}$ axis). In the other direction ( $\mathrm{Y}$ axis), the TRR alignment remains stable. The original Schmidt plate mount from ChemCam was changed with the installation of Belleville washers in the axial direction and a pseudo-kinematic mount in the radial direction. There is still a residual thermo-elastic effect, with a hysteresis depending on the sign of the temperature gradients. Mechanical environments (vibrations and shocks) cause the Schmidt plate to move away from its optimal position because of friction. Numerous laboratory tests have shown that several hot-cold cycles, similar to the day-night temperature range, will reset the Schmidt 
Fig. 48 Mast-Unit transmission to the mast-to-body fiber (top), to the IRS (middle), and to the RMI (bottom). In each case, the red line is the requirement
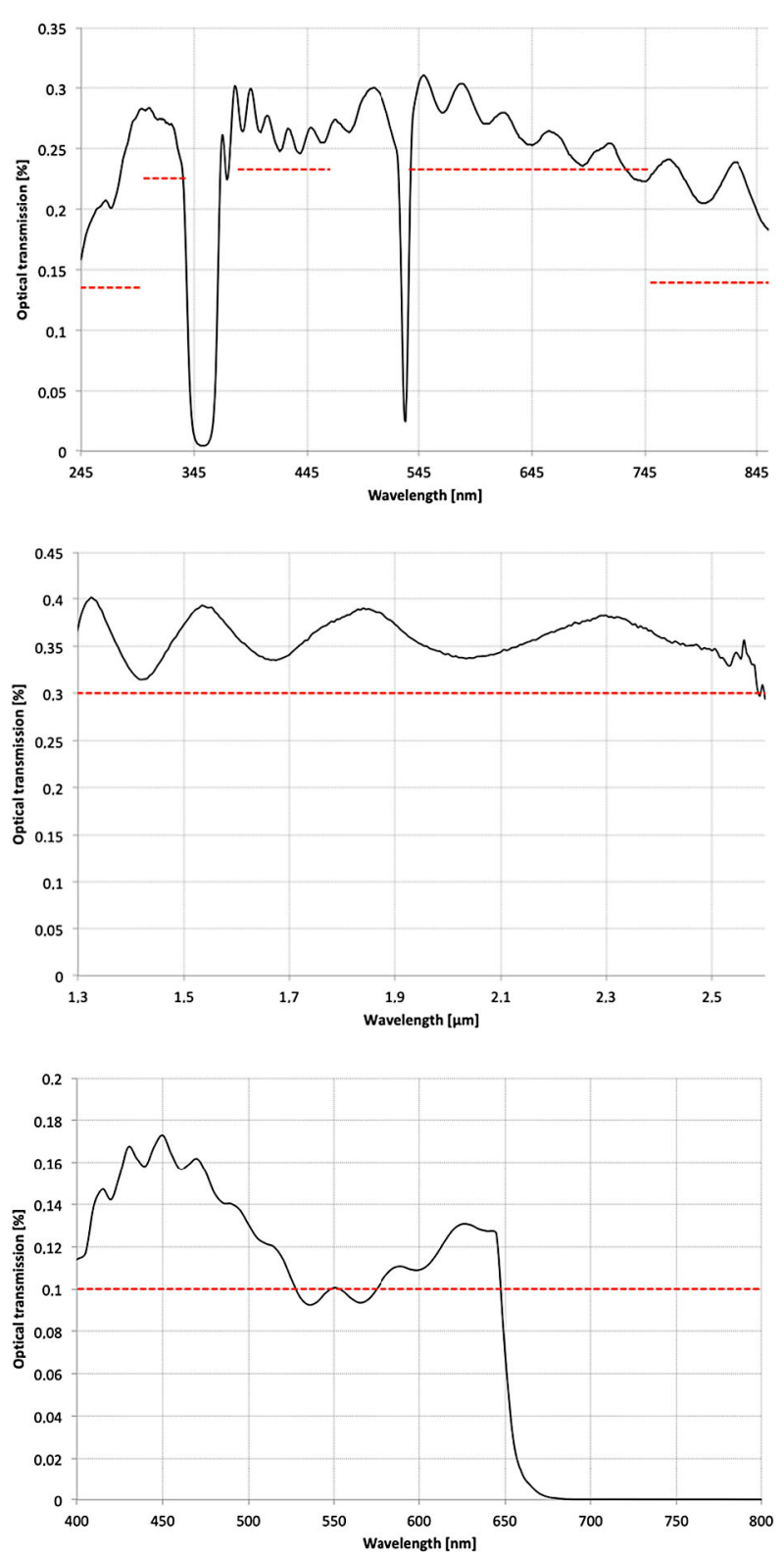

plate into its rest position. In the end a bias was introduced to optimize the alignment at $-25^{\circ} \mathrm{C}$. The TRR alignment underwent a final check in ATLO at JPL after mechanical and thermal system tests. The results showed that the fiber FOV covers the green laser beam from $-30{ }^{\circ} \mathrm{C}$ to $+10{ }^{\circ} \mathrm{C}$ and up to 7 meters (Fig. 49).

For the IRS, the specification is also $\pm 0.35 \mathrm{mrad}$ from the telescope boresight. Its alignment is determined by the mechanical adjustment of the IR periscope (Sect. 4.3.3). Measurements over temperature show a drift $<0.3 \mathrm{mrad}$ over the $\left[-40^{\circ} \mathrm{C} ;+20^{\circ} \mathrm{C}\right]$ temperature range. 


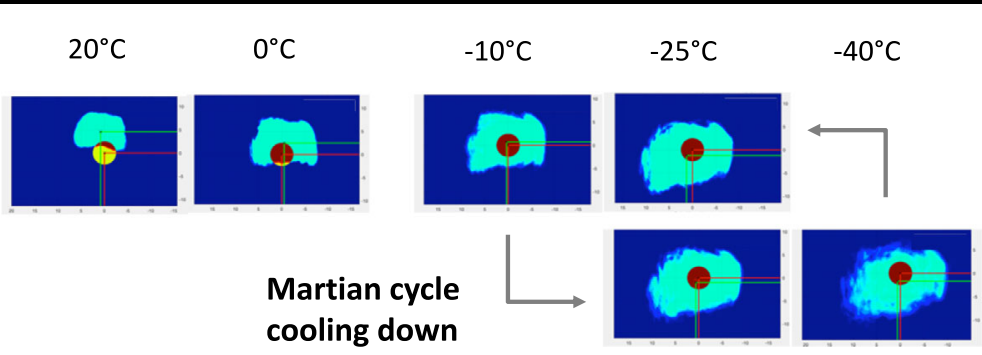

Fig. 49 Alignment of the green laser beam at $7 \mathrm{~m}$ and the fiber FOV at different temperatures; arrows indicate the direction of the thermal changes. In green, the position of the green-beam centroid; in red the centroid of the fiber

Fig. 50 Co-alignment of SuperCam investigation axes. The relative positions of the centers are independent of distance. (Black) Center of the RMI field of view. (Red) Center of the LIBS pit. (Green) Footprint of the green laser beam at two distances and $-30^{\circ} \mathrm{C}$. (Grey) IRS center and FOV. (Blue) Collect by the mast-to-body fiber; this is the actual FOV of TRR/L and VIS. The requirement is to have the LIBS, IRS, mast-to-body centers within $0.35 \mathrm{mrad}$ of each other, the radius of the mast-to-body fiber FOV

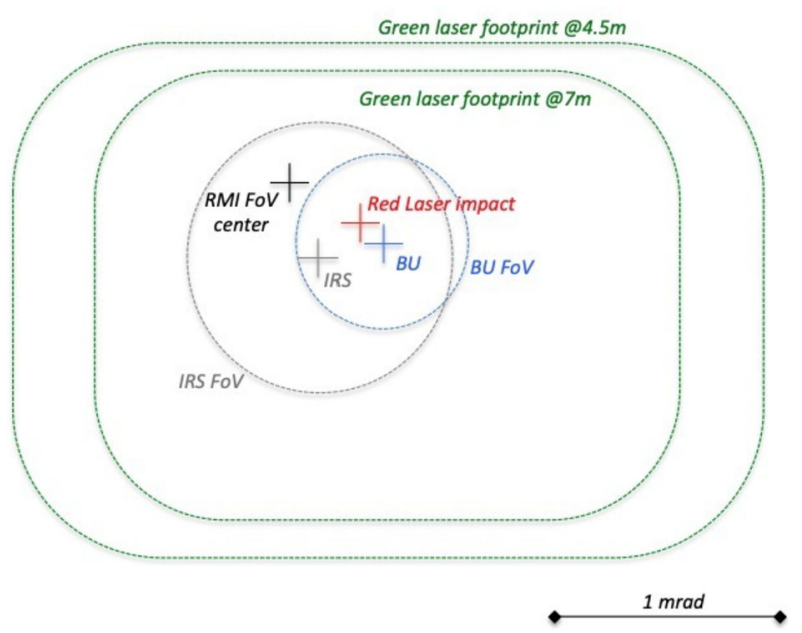

The RMI has a large (compared to the fiber) $18.8 \mathrm{mrad}$ FOV. The specification was to be aligned within $2 \mathrm{mrad}$ of the other techniques, which is obtained by adjusting the camera cube along the CMOS axis at the rear of the Beamsplitter.

Figure 50 summarizes the measurements obtained for the flight unit during final acceptance tests. We know that alignments are very stable with temperature (see above), and they are independent of distance to the target. As a reminder, the projection of the Body-Unit fiber defines the telescope boresight; its FOV is $\sim 0.7 \mathrm{mrad}$ (see next section for details). Hence the analytical investigations (LIBS, VIS, IR, and TRR/L) are grouped within $0.35 \mathrm{mrad}$, as requested. The center of the imager FOV is $\sim 0.2 \mathrm{mrad}$ away from the center of the detector, better than the $1 \%$ requirement. The fiber FOV is within the green laser beam footprint at $-30{ }^{\circ} \mathrm{C}$ for all distances, which was obtained after several design iterations (see Sect. 4.5.2, second paragraph, and Fig. 49).

\subsubsection{Fields of View and Depths of Field}

The fields of view induced by this optical design are essential inputs for the team to analyze the data and the origin of the signals. The fiber and the IR periscope are effectively small pinholes with varying efficiencies across their diameters. This coupling efficiency defines $d e$ facto the LIBS and TRR FOV (Fig. 51 left). At FWHM, the fiber FOV is $0.67 \mathrm{mrad}(\mathrm{H}) \times$ 
Fig. 51 Relative coupling efficiency within the Beamsplitter, at the entrance of the mast-to-body fiber (top) and IRS (bottom) in the horizontal (squares) and vertical (circles) directions. The FOV requirements are indicated in red, $0.8 \mathrm{mrad}$ for the fiber, $1.2 \mathrm{mrad}$ for the IRS. Efficiencies are normalized at the center of the fiber and IR periscope
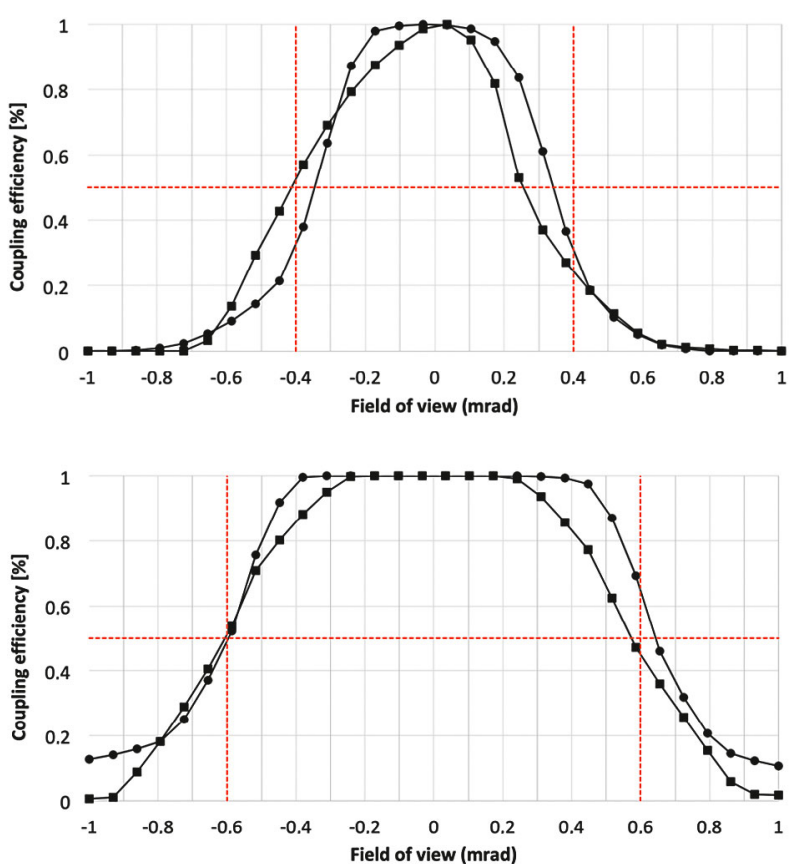

$0.70 \mathrm{mrad}(\mathrm{V})$. The diameter of the LIBS plasma on Mars is typically $\leq 1 \mathrm{~mm}$ (Maurice et al. 2012). Assuming the largest size (1 mm), the plasma image should be well collected by the fiber at the distance of the SCCTs if the laser and fiber are well centered. Beyond this distance, the LIBS FOV is smaller than that of the fiber. Since the green beam is $\geq 1 \mathrm{~cm}$ on targets, the fiber FOV is, in fact, the TRR FOV. For the IRS, the FOV size is $1.18 \mathrm{mrad}$ by $1.24 \mathrm{mrad}$ (Fig. 51 right), therefore $\sim 70 \%$ larger in each dimension than for the laser investigations. See Sect. 4.3.2 for the RMI FOV (18.8 mrad). Figure 50 illustrates the overlap between the IRS and the fiber FOVs. The former covers more than $90 \%$ of the latter.

The Depth of Field (DOF) is another essential parameter of each technique's performance. To obtain an estimate of the DOF, we calculate a criterion for different distances a few percent closer and farther than the exact distance to the target (Fig. 52). For LIBS and TRR collects, an optical design code was used to simulate the coupling efficiency at the center of the fiber. For the IRS, the coupling efficiency is calculated with regard to the center of the pinhole at the entrance of the IR periscope. For the RMI, the performance criterion is the contrast for an MTF at 20 cycles per mm. And for the creation of the LIBS plasma, we calculate the irradiance on target. Of course, those performance criteria cannot be readily compared, but they allow us to find out what technique is the driver to focus the telescope. Figure 52 displays this information at $2 \mathrm{~m}, 7 \mathrm{~m}$, and $30 \mathrm{~m}$. In all cases, the IRS is relatively insensitive to small distance variations around the best focus. The same is true for the fiber collect inside $7 \mathrm{~m}$. However, for the RMI, the contrast drops by $50 \%$ beyond $\pm 0.25 \%$ of the best focus at $2 \mathrm{~m}$, beyond $\pm 0.6 \%$ at $7 \mathrm{~m}$, and beyond $-2 \%$ or $+3 \%$ at $30 \mathrm{~m}$. The profile for the plasma irradiance is similar to the RMI's. Thus, the RMI and plasma generation have the most stringent requirements for focus, which was set to $\pm 0.4 \%$ of the distance to the target. 
Fig. 52 Relative depths of field at $2 \mathrm{~m}$ (top), $7 \mathrm{~m}$ (middle), and $30 \mathrm{~m}$ (bottom) for different performance criteria - see text for their definition: IR collect (blue), Body Unit collect (grey), RMI (orange), plasma creation (yellow). The horizontal axis is for the variations of the focus distance with regards to the target distance
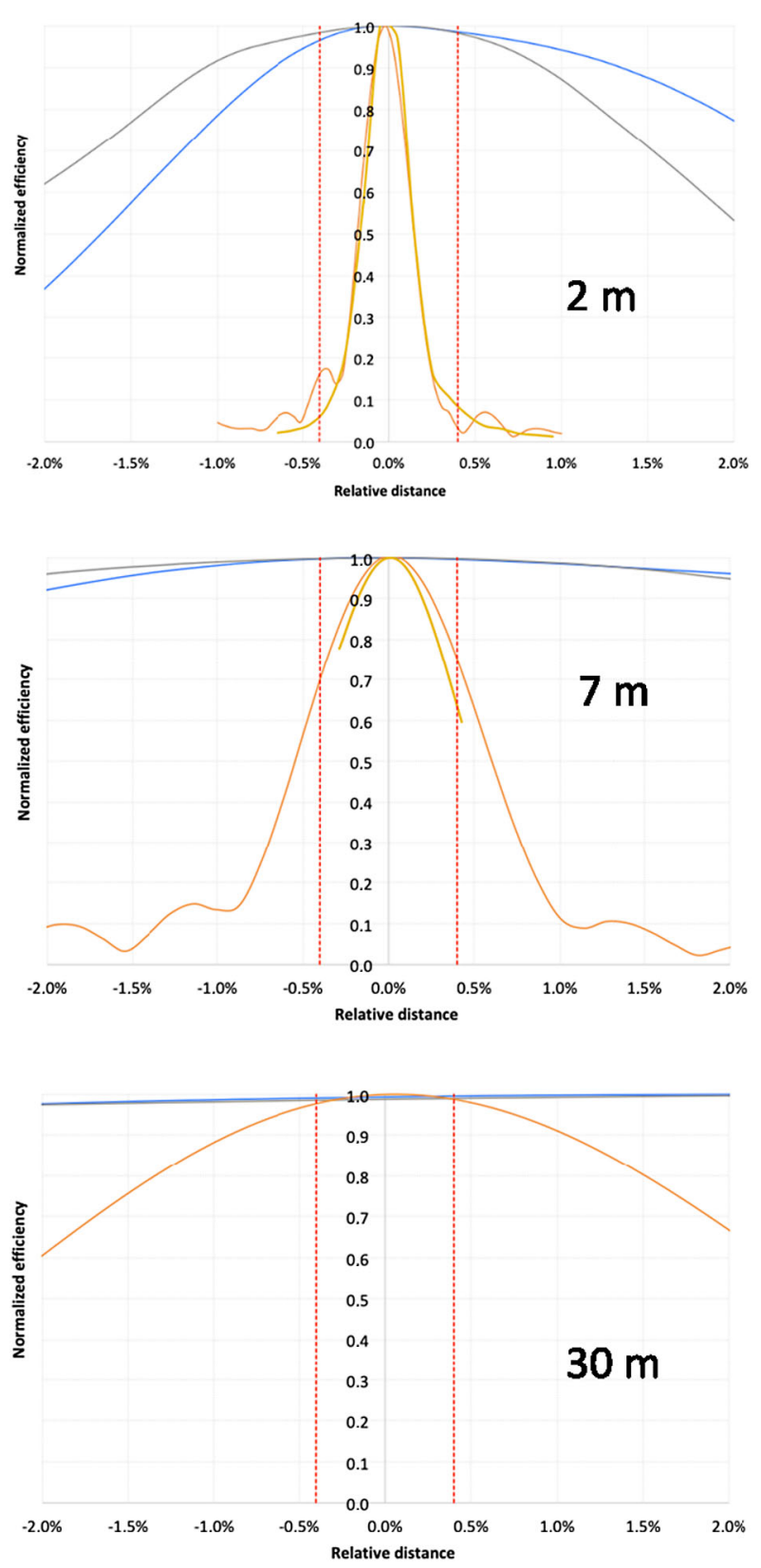

\subsection{Mechanical Design}

The Mast-Unit mechanical design is mostly inherited from ChemCam's, with additional functions (TRR/L, IR, MIC) to be added without changing key resources (mass, volume). Finite element analyses and feedback on the qualification of ChemCam subsystems showed that there were margins that could be used without reducing the strength of the structure as a whole. We also used new manufacturing processes, titanium alloy for all fasteners, flex 


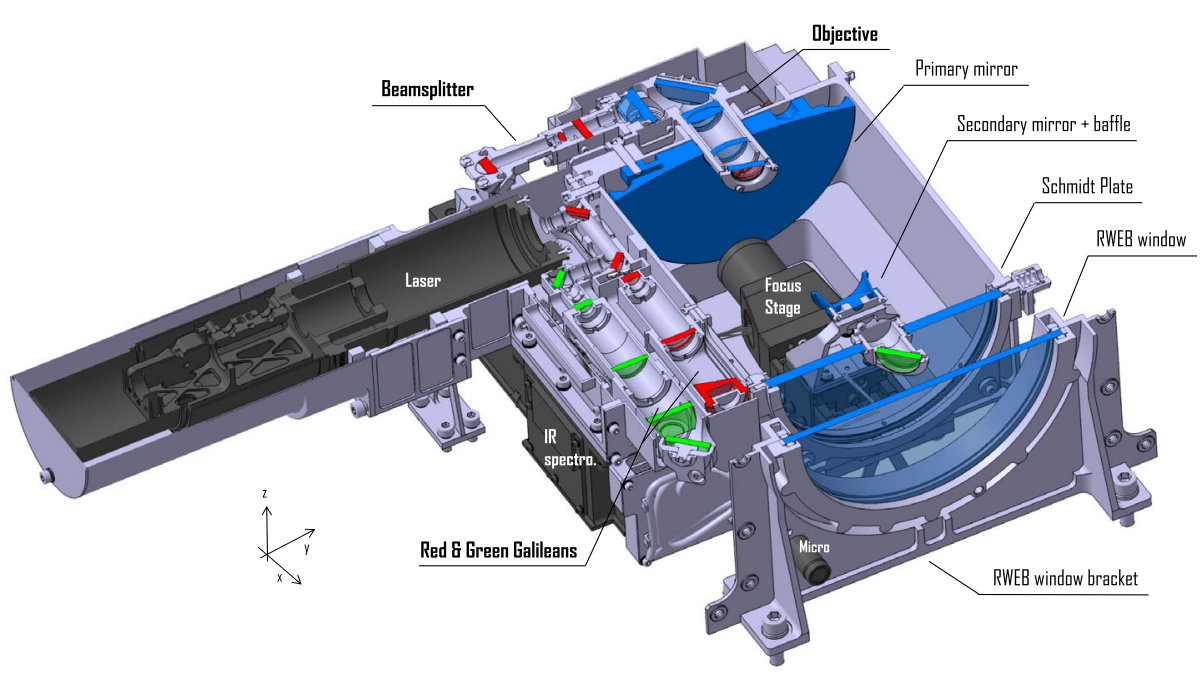

Fig. 53 Cross section of Mast-Unit OBOX (some parts are not shown). In black the integrated subsystems. Color coding of the optics corresponds to optical design of Figs. 43 and 44

harness rather than cables, and a few optimizations of optomechanical mounts. In the end, the mass-to-volume ratio increased by $5 \%$ for the same envelope.

\subsubsection{Mechanical Architecture}

The OBOX and EBOX are mechanically independent of one another. In practice, each box has separate and independent 3D CAD files, designed using primarily CATIA by Dassault System (France). They were merged only for accommodation studies on the rover mast and for electronic interconnection designs.

The OBOX was built around a structural optical bench, which carries all of the optical components and major subsystems. For orientation, the $\mathrm{x}$-axis is along the telescope boresight, the $\mathrm{y}$-axis close to the laser axis, and the $\mathrm{z}$-axis is directed upward. Figure 53 is an $\mathrm{x}-\mathrm{y}$ section at the center of the primary mirror. Most optics lie in this plane. At the rear of the telescope, to redistribute light for the different techniques, the Beamsplitter lies in the $\mathrm{y}-\mathrm{z}$ plane (Fig. 54). As it is made of aluminum, the dimensions of the optical bench vary with temperature. We have tried to make the thermal expansion as symmetrical as possible. The only active correction is performed by the translation stage that refocuses the telescope at each new target distance but also copes with thermal expansion. This overall architecture is very similar to ChemCam's, except for the green Galilean and the infrared spectrometer.

The EBOX contains most of the Mast-Unit electronics (Fig. 55). The various boards are inserted vertically. Compared to ChemCam, the EBOX density has increased thanks to flex harnesses (instead of cables) and a more compact electronic design. We note that the laser board is equipped with a Faraday cage around the high voltage. It is made of Ultem 2300 covered by a Cu coating with a gold coat. The IR board is mounted inside a dedicated housing.

\subsubsection{Mounting Principles}

Figure 56 is an exploded view of the Mast-Unit telescope, and Fig. 57 shows the associated photographs. Parts like the two Galilean telescopes (Fig. 58) and the beam splitter illustrate 


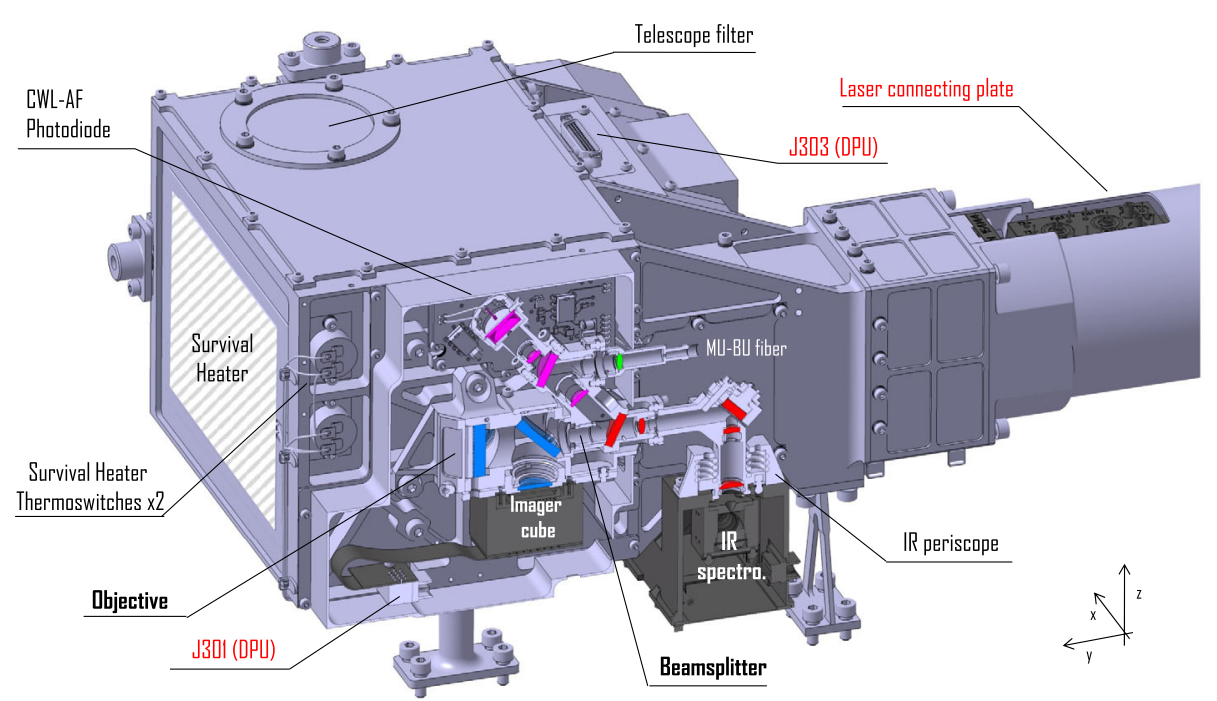

Fig. 54 Rear of the Mast-Unit OBOX. Section of the objective and beamsplitter, which includes the IR periscope. Color coding of the optics corresponds to optical design of Figs. 43 and 44. Connectors are in red (refer to Fig. 55 for details)

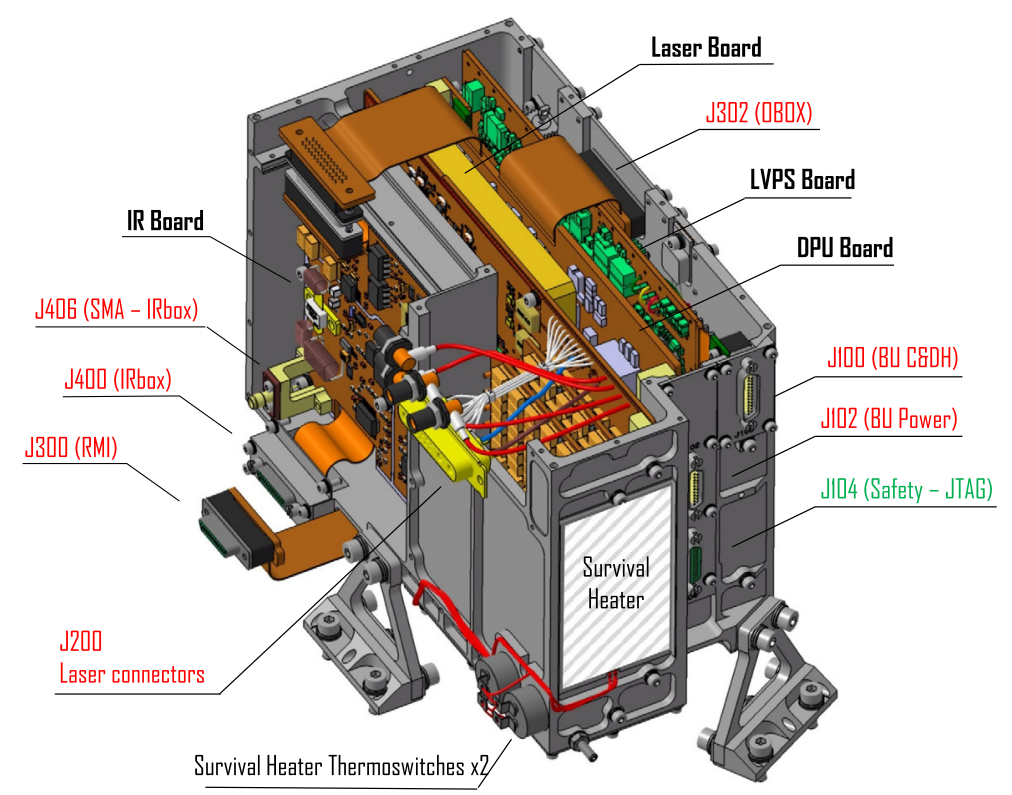

Fig. 55 Open Mast-Unit EBOX. Connectors are in red, except for the ground plug in green. Covers are not shown

the mounting principles that we have chosen for optics that need tight alignment and hence need to be adjustable. Mechanical parts, mostly barrels, were machined with better than $10 \mu \mathrm{m}$ general tolerances. The precision is lowered to a few microns on contact surfaces, like 


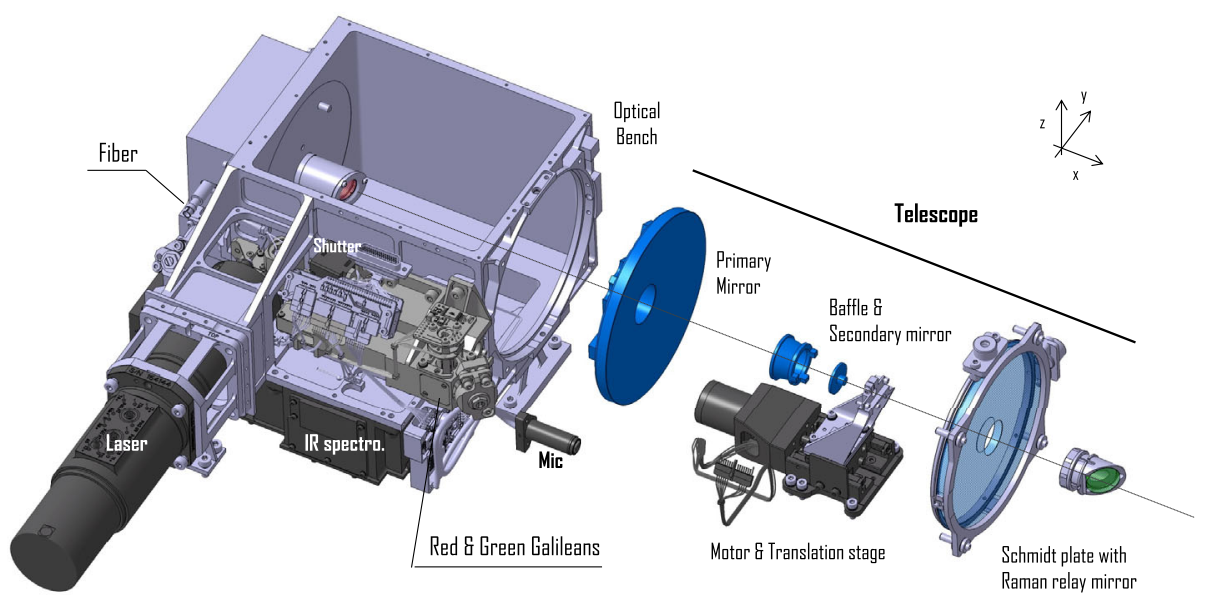

Fig. 56 Exploded model of the Mast-Unit telescope (covers not shown). In black the integrated subsystems that are presented Sect. 4.3
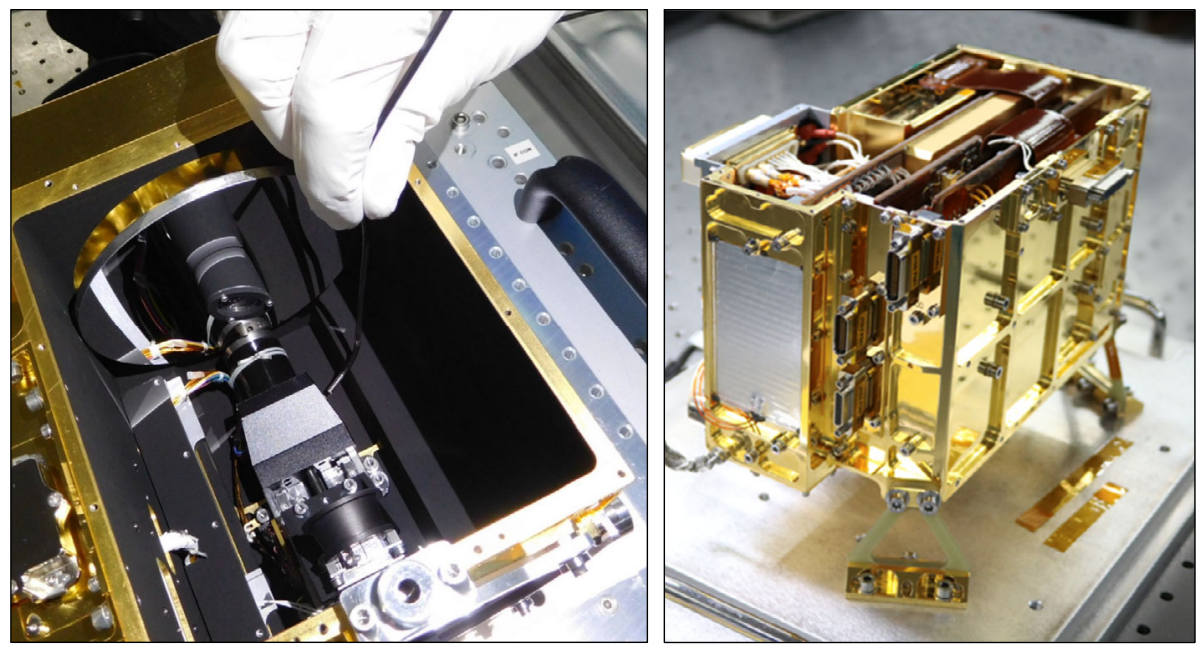

Fig. 57 Details of the Mast Unit. (Left) interior of the telescope. (Right) EBOX with a special view on the survival heater and external connectors

that of the primary mirror. Lenses and shims are paired together to minimize stress while maintaining their alignment. Specific spacers with appropriate CTE values were used to compensate for thermal effects. For the mirrors, spring washers like Borrelly ${ }^{\mathrm{TM}}$ or Belleville washers reduce the thermal deformation of the optical surfaces. The final alignments were obtained with adjustment pins, adjusted shims, or spacers.

Some optics have specific thermo-elastic mounting. The laser dichroic, for instance, was glued on its perimeter to avoid stress and strain on the optical surface with multiple layers of coatings. The most challenging mounting was the Schmidt plate positioning at the front of the instrument, and its stability with temperature. There are four $1 \mathrm{~cm}$ long flat spots at $90^{\circ}$ along the circumference of the Schmidt plate. On two of them, next to each other, are 


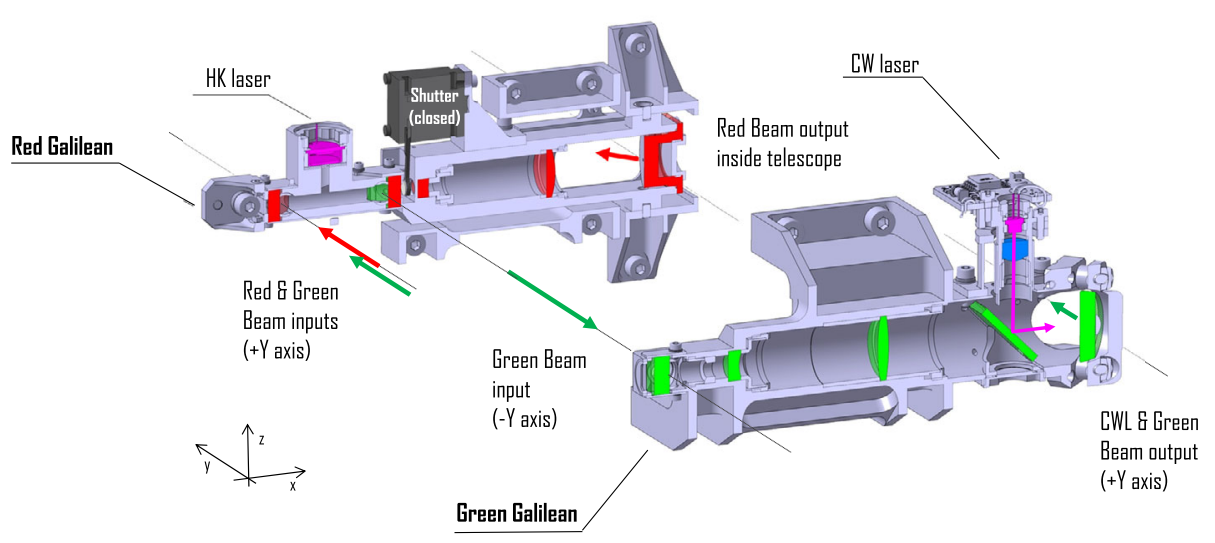

Fig. 58 Sections of the Mast-Unit Galilean telescopes. Color coding of the optics corresponds to optical design of Figs. 43 and 44

bonded V-groove ( $-\mathrm{Z}$ direction) and spherical (-Y) Invar 36 shims. On the opposite sides $(+\mathrm{X}$ and $+\mathrm{Z})$, two Ti plungers with springs are pushing the Schmidt plate against a reference pin and a flat face on the optical bench. The plungers are mounted between thin Teflon shims; a clamp with Belleville washers at the front minimizes stress from vibrations, shocks, and thermal gradients. A $\sim 0.4 \mathrm{mrad}$ hysteresis loop results from this assembly over a $40{ }^{\circ} \mathrm{C}$ range. At the center of the Schmidt plate, the folding mirror is bonded inside an Invar 36 barrel. The hysteresis loop induces a rotation of this mirror, followed by the divergence of the green beam with respect to the telescope boresight. After several iterations, we found a configuration that is compatible with the alignment requirements at cold temperature, over the entire lifetime of the instrument (see Fig. 49).

\subsubsection{Material and Coatings}

The optical bench, covers, EBOX, and most of the optomechanical parts were manufactured from Al7075 T73 51. This grade of aluminum alloy is easy to machine with tight tolerances. It offers the best compromise between resistance, mass, and stiffness. Some other parts (mounting feet of the OBOX, laser, and CWL supports) were made of polished Titanium alloy TA6V. This material isolates the parts thermally and offers sufficient strength characteristics for vibration and shock tests. The mounting feet of the EBOX are built from Glass Epoxy (G10) to insulate the box from the camera bar. The design was driven by optimization between conductance, stiffness, and mass. A small chip of SPECTRALON is clamped at the tip of the shutter blade to stop the red beam during TRR/L. Finally, the autofocus and laser housekeeping (HK) photodiodes are placed between two spacers in ULTEM 2300, to keep the signal ground isolated from the chassis ground.

Different coatings were implemented. Gold coating, with a $15 \mu \mathrm{m}$ nickel layer under $<2 \mu \mathrm{m}$ of gold, was deposited on polished external surfaces, to increase their emissivity. This coating reduces thermal radiation from the RWEB cover. To avoid stray light, we used black PNC thermal coating by MAP (France), along the light path, despite its fragility throughout the integration phases. For structural parts, which are not along the optical path, Surtec 650 was applied in the place of Alodine. For SuperCam, CNES product assurance experts have qualified that the gold coating could be covered with PNC, and they have also defined how Surtec was deposited on A17075. 
Fig. 59 Front view of the Mast Unit. (Credit: CNES/OLLIER Alexandre, 2019)

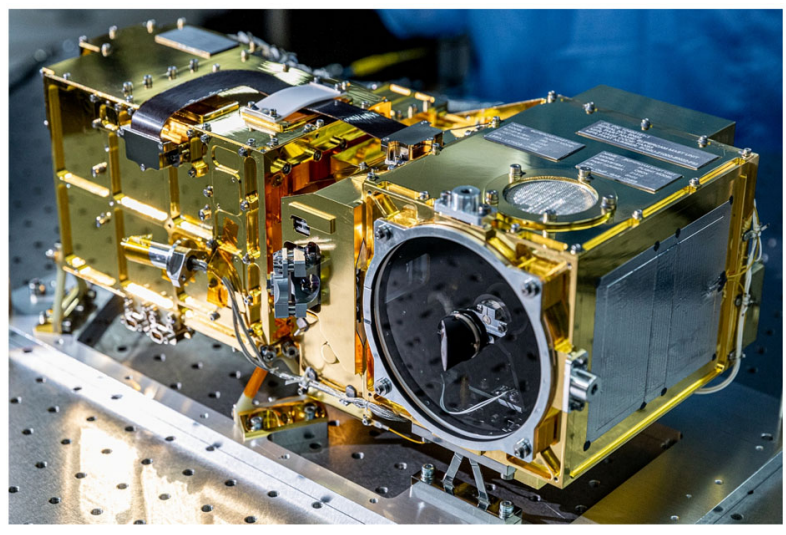

Fig. 60 Rear view of the Mast Unit. (Credit: CNES/OLLIER Alexandre, 2019)

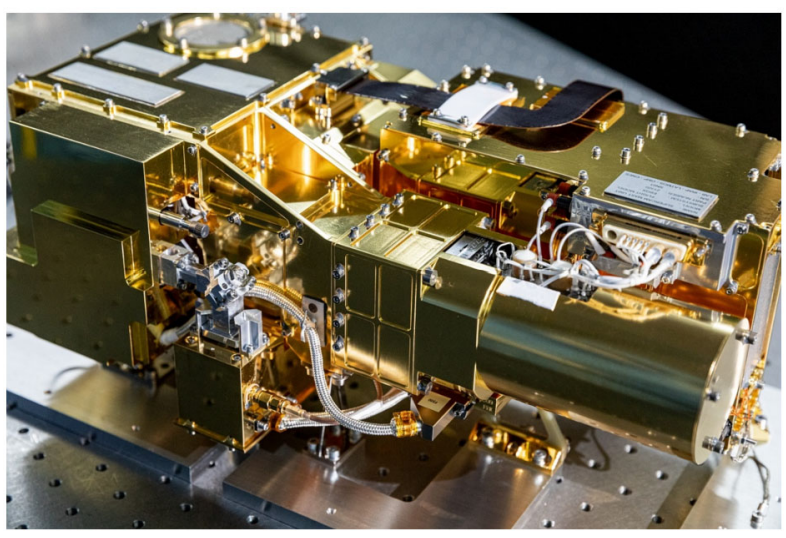

With respect to epoxies, the standard EC2216 was used for bonds that are not sensitive to thermal gradients and as thread reinforcement on fasteners. More specialized glues were used too. Invar 36 shims for kinematic mountings are bonded with Epotec301. This glue has very low viscosity but its coefficients of thermal expansion are close to those of Invar and fused silica. The laser dichroic was bonded with NuSil SCV-2585 RTV glue, which is a silicone glue with ultra-low outgassing properties. Last, adhesive Teflon tape covers the edges of protruding angles to protect the cable and flex harnesses.

Except for black painted areas, each mechanical part was cleaned by ultrasonic baths, isopropyl alcohol, and thermal bakeout. Humidity control and contamination witnesses were used all along the integration and test campaign.

The FM unit is shown prior to delivery in Fig. 59 and Fig. 60.

\subsubsection{Finite Element Analysis and Tests}

Given the similarity with ChemCam (mass, dimensions), the Mast-Unit structure does not a priori present any risk for survival of the mechanical environments. However, the new additions (IRS, TRR/L parts, shutter, etc.) induced subtle changes (e.g., deflection of the mirror at the center of the Schmidt plate) that had to be carefully taken into account.

For mechanical tests, specifications were negotiated with the project for quasi-static loads, vibrations (first natural frequency, random vibrations), and shocks: 
Fig. 61 Random vibrations of the Mast-Unit FM along the $\mathrm{Z}$-axis. Primary notching is not implemented along this axis. (Top) Qualification requirement and actual excitation input, including secondary notching. (Bottom) Resulting forces for the OBOX and EBOX. Quasi-static loads are given at the instrument interface
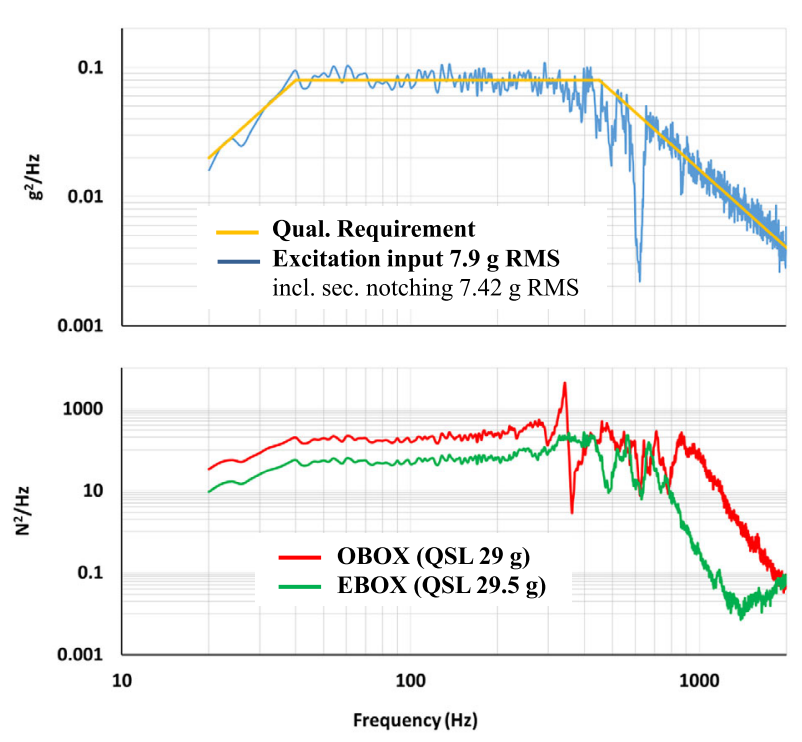

- Launch is the most stressing phase of the mission for the quasi-static loads. The specification was given at the center of gravity along each axis $(\mathrm{X}, \mathrm{Y}, \mathrm{Z})$ of the instrument: (31, $12,17) \mathrm{g}$ for the OBOX, $(26,19,19) \mathrm{g}$ for the EBOX.

- The specification for the first resonant frequency was $120 \mathrm{~Hz}$. At qualification levels, the random vibration stress at the mounting feet of the instrument was a plateau at $0.08 \mathrm{~g}^{2} / \mathrm{Hz}$ with $(7.9 \mathrm{~g} \mathrm{rms})$ for two minutes. The acceptance level was $3 \mathrm{~dB}$ below and $1 \mathrm{~min}$ duration. SuperCam FM adopted a protoflight approach: qualification levels but flightacceptance duration.

For the $\mathrm{X}$ and $\mathrm{Y}$ axes, a primary notching (force limiting using force gauges) was defined for the EBOX and OBOX, independently of one another, since the two boxes are mechanically decoupled but still connected by a stiff harness. A single excitation profile merged these two notchings. Their intensity was defined as a function of the instrument mass, its first frequency, plus an adjustment coefficient. The notching was measured directly by force gauges during the test. Their integration shifted the overall center of gravity; this geometrical change was taken into account in our analyses. In addition, to protect critical subsystems such as the laser and focus stage, a secondary notching (response limiting) was applied along the Z-axis (not to exceed $-12 \mathrm{~dB}$ per JPL standards).

Figure 61 illustrates the qualification of the flight-unit for random vibrations along the $\mathrm{Z}$-axis. The excitation was performed for one minute. The power spectrum of the forces at the interface of the camera bar was given for each box. The notching of the focus stage at $13 \mathrm{~g} \mathrm{RMS}$ drastically reduced the response (and the input baseline) at $900 \mathrm{~Hz}$, and between 300 and $600 \mathrm{~Hz}$. The notching of the EBOX at $16 \mathrm{~g}$ RMS dropped the response around $600 \mathrm{~Hz}$. There is a structural resonance/anti-resonance at $400 \mathrm{~Hz}$ for the OBOX. The quasi-static load requirement (above) was met without difficulty along the Z-axis.

- Standard shock profiles are too stressful for critical hardware, such as the laser, IR photodiodes, and Schmidt plate assembly. Hence, to refine the shock specifications, we performed a "real" mast deployment at JPL on a rover test bench, the most stressful source of shock. Based on that input, most subsystems were shocked twice per axis; the Mast-Unit EQM was shocked once; the FM was not shocked. 
The first finite element analysis was performed on the STM. It did not reveal any issue on structural elements. These results are used for an early definition of the notching. Subsequent STM tests were performed under force limiting conditions at the interface. Besides validation of the structure, STM tests helped to refine specifications for the various subsystems. Some were tested on rigid interfaces, while others were mounted on the STM to benefit from the notchings of the instrument in random vibration and the damping of shocks by the structure. The IRS is a good example: the whole structure was tested on a rigid interface, but the photodiodes were mounted on the STM for their own shock qualification tests. The STM thus helped to test the COTS parts and was used to qualify the Schmidt plate mounting. Altogether, STM has performed nine hours of random vibrations over 21 test campaigns and 140 shocks!

A new finite element model was built for the EQM, accounting for the updated behavior of the model, for instance, the primary notching of the instrument. These analyses suggested that we needed to strengthen the Ti supports of the CWL and the RMI. As a result of these design modifications, a final model was built to assist the EQM qualification tests. Random vibrations were carried out on force gauges, with primary and secondary notches.

Post mechanical and thermal tests yielded significant misalignments of the red and green laser beams. We proposed a new assembly of the Schmidt plate (see Sect. 4.5.2, second paragraph), which was validated by non-linear finite element simulations (friction, contact). To back up the simulations, we performed several fretting tests. The new configuration was first mounted on the STM; the rotation of the Schmidt plate was measured by a stereocorrelation technique. The final configuration was mounted on the EQM, and tested at JPL at the time of the mast deployment. After several iterations, and the introduction of a bias at cold temperature, the Schmidt plate assembly was qualified.

For the FM, the finite element model stayed the same, but it was rescaled without force gauges, thanks to sinewave data on a rigid interface. The first Eigen frequencies of the Mast Unit in each direction are above $180 \mathrm{~Hz}$. The project performed predictive analyses with SuperCam coupled to the rover to ensure that levels seen during random vibrations would not exceed the qualification levels. The Mast Unit passed random vibrations on the rover successfully, with levels $<-12 \mathrm{~dB}$ compared to the instrument qualification.

\subsection{Thermal Design}

The Mast Unit is generally insulated inside the Remote Warm Electronics Box (RWEB). Beyond passive measures, there are survival heaters on the OBOX and the EBOX to maintain all systems within their non-operational Allowable Flight Temperatures (ATFs, Table 6) thanks to mechanical thermostats. Active thermal controls were also implemented to optimize the performance of some systems, including three warmup circuits for the lasers and TECs for the IR photodiodes.

\subsubsection{Thermal Architecture}

The overall thermal architecture is based on the following concepts to meet the mission requirements (Fig. 62):

- The MU is thermally insulated from the camera bar, which can go down to $-110{ }^{\circ} \mathrm{C}$. The OBOX is mounted on three titanium feet, the EBOX on three epoxy glass bipods.

- There is a $2 \mathrm{~cm}$ thermal gap (Mars atmos.) between the Mast Unit and the RWEB. Per JPL experience, this gap is big enough to limit the conduction, and small enough to prevent any convection. 
Table 6 Allowable Fight Temperatures (AFT) ranges for the different subsystems, operational and non-operational, including a restricted range for some subsystems

\begin{tabular}{lc|c|c|c} 
& \multicolumn{2}{c}{ Operational } & \multicolumn{2}{c}{ Non-operational } \\
& min & max & min & max \\
\cline { 2 - 5 } Allowable Flight & Temperatures & & & \\
(default) & -40 & 35 & -40 & 40 \\
Laser & -35 & 25 & -40 & 40 \\
Focus table & -35 & 35 & -40 & 40 \\
Microphone & -128 & 50 & -128 & 50 \\
Restricted Performance Range & & & \\
Laser & -30 & 10 & & \\
IRS & -40 & -5 & & \\
Focus table & -30 & 35 &
\end{tabular}

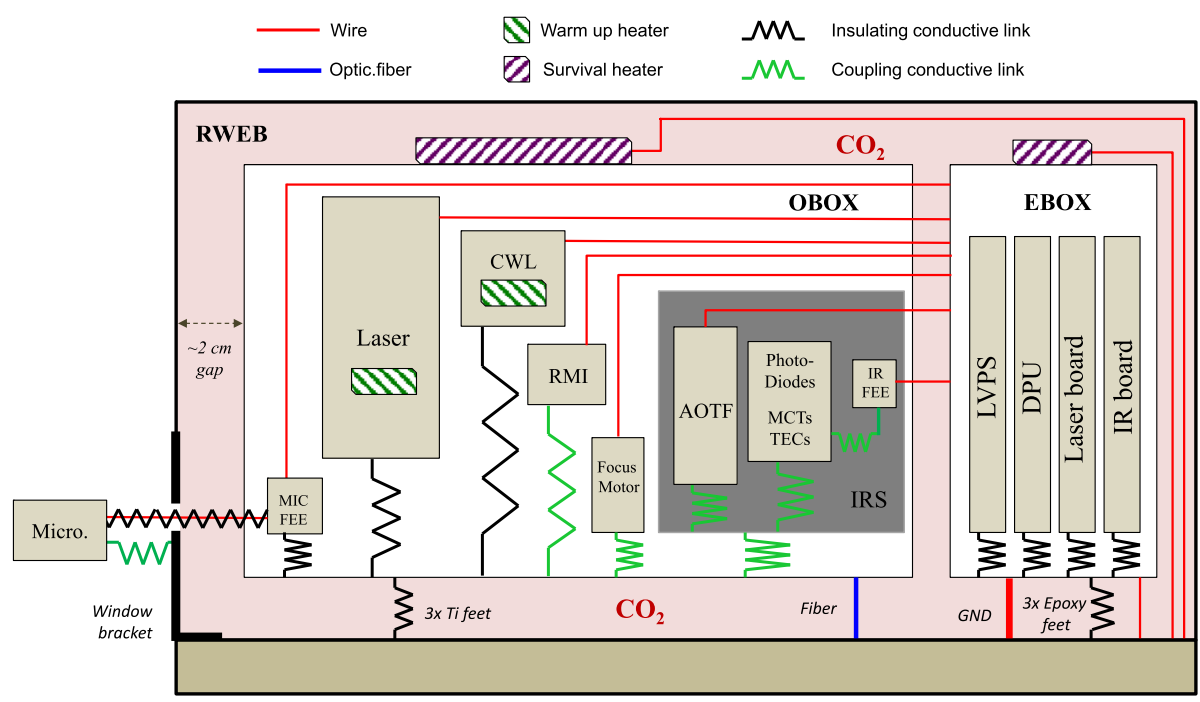

Fig. 62 Schematic of the Mast-Unit thermal architecture

- External surfaces of the OBOX and the EBOX were polished gold to limit radiative heat transfer. The interior of the telescope, which is visible from the outside, was painted black.

- The OBOX and the EBOX have independent survival heaters to keep them above $-40{ }^{\circ} \mathrm{C}$ at all times. These heaters are controlled by two thermo-mechanical thermostats wired in-series, which were provided by JPL.

- The CW laser is thermally insulated from the rest of the Mast Unit. It has its own warmup heater.

- The secondary mirror of the telescope is surrounded by a thermal baffle, which absorbs heat when looking toward the Sun.

- The pulsed laser is thermally insulated from the rest of the Mast Unit via a titanium mounting structure. It has its own warmup heaters.

- There are several harnesses (flex and cables) between the EBOX and the OBOX. The OBOX has no harness to the rover except the fiber optic cable. The EBOX has several harnesses to the Rover and a ground stud to the camera bar.

- The microphone is mounted outside the RWEB; it is thermally coupled to the RWEBwindow bracket on the camera bar. It is linked by Manganin ${ }^{\circledR}$ wires to its FEE board to minimize the thermal conductivity of the harness between the RWEB and the OBOX. 
To monitor the thermal status of the Mast Unit, there are four Platinum Resistance Thermometers (PRTs). Their location is important: on the pulsed laser mounting interface, on the secondary mirror bracket, on the IRbox interface with OBOX, on the LVPS board. They are read by the rover RCE at all times, even when the instrument is off. In addition, there are a total of 17 temperatures read by the Mast Unit itself. Seven of them are used by the Mast Unit to control in real time the laser and CWL warmups, as well as the IRS TECs. The Body Unit also monitors the Mast-Unit temperatures to check that AFTs are met, and to adapt the configuration parameters, including the speed of the focus mechanism and laserstack pumping current as a function of the OBOX temperature. Note that the Body Unit also controls the telescope focusing and offset laws that depend on the OBOX temperature.

\subsubsection{Thermal Analysis}

The RWEB (and camera bar) is the Mast Unit's outer skin. It is heated by the Sun, scattered light from the atmosphere, and sunlight reflected by the ground. It is cooled by radiation to the ground and to the sky, by convection with the atmosphere, and by conduction to the rover mast. Considering the material characteristics and their thermo-optical properties, thermal analyses (worst-cases, operational timeline) were conducted at different times during the project to account for the evolution of the design, operational and non-operational configurations of the Mast Unit. All analyses were mainly based on the EQM configuration.

We have defined seven worst-case scenarios (Table 7) with the following assumptions: the thermal environment was set at $26.4^{\circ}$ latitude South (Holden crater). For cold cases, Mars heliocentric longitude was $\mathrm{L}_{\mathrm{s}}=91$ (winter) and the RSM is pointing South. For hot cases, $\mathrm{L}_{\mathrm{s}}=259^{\circ}$ (summer), the RSM had different pointing orientations. The first case was a pure steady state, while others were cycling states over a period of $24 \mathrm{~h}$. When the Mast Unit was on, the warmup time was a maximum of 20 minutes, if needed. For the power dissipation, an ambitious timeline (all techniques, $3 \times 3$ raster, depth profile, etc.) was made, using between $4.3 \mathrm{~W}$ (standby) and $34 \mathrm{~W}$ (RMI autofocus) for two hours.

Cold cases ( 3 scenarios) were used to make sure that the Mast Unit stayed above the minimum AFT, while sizing survival and warmup heaters. Pre-scaling studies concluded that two heaters are required to maintain the EBOX and the OBOX above $-40{ }^{\circ} \mathrm{C}$. We opted for single layer polyimide thermofoil heaters by Minco: $29 \mathrm{~cm}^{2}(15.7 \mathrm{~W}$ at $28 \mathrm{~V})$ for the EBOX, and $77 \mathrm{~cm}^{2}(25.4 \mathrm{~W}$ at $28 \mathrm{~V})$ for the OBOX. The case was similar for the laser heaters: early studies concluded that both the pulsed and CW lasers needed to be insulated from their mounting structure, and that dedicated warmup heaters were necessary. For the latter, we chose $2.4 \mathrm{~cm}^{2}(1.5 \mathrm{~W})$ for the stack, and two $1.4 \mathrm{~cm}^{2}(0.5 \mathrm{~W})$ heaters for the doubler. For the CWL, we chose $2.15 \mathrm{~cm}^{2}(1.4 \mathrm{~W})$.

- The first cold case was a steady-state with maximum energy demand to check the dimensions of the survival heaters. The simulations showed that $3.5 \mathrm{~W}$ and $8.7 \mathrm{~W}$ are required to maintain the EBOX and $\mathrm{OBOX}$ above $-40{ }^{\circ} \mathrm{C}$, respectively. This total heating power of $12.2 \mathrm{~W}$ is compliant with the $20 \mathrm{~W}$ not-to-exceed allocation given by the project.

- The second cold case ran over $24 \mathrm{~h}$ to evaluate the survival heaters' duty cycle. During the coldest part of the sol, with $22 \mathrm{~V}$ on the primary line (e.g., lowest possible voltage provided by the rover), the maximum duty cycle was $39 \%$ for the EBOX and $64 \%$ for the OBOX, below the $80 \%$ project specification. Over the full sol, the energy was 78 W.h and 191 W.h, respectively.

- For the last cold case, the instrument was operating for two hours, starting at 7:20 in the morning, local Mars time. The warmups started at 7:00. It took $6.4 \mathrm{~min}$ for the CWL to reach $-10{ }^{\circ} \mathrm{C}$ (slope $4.5^{\circ} \mathrm{C} / \mathrm{min}$ ), $4.3 \mathrm{~min}$ for the laser stack to reach $-30{ }^{\circ} \mathrm{C}$ (gradient 
$1.5^{\circ} \mathrm{C} / \mathrm{min}$ ), and $2.8 \mathrm{~min}$ for the doubler to reach $-30{ }^{\circ} \mathrm{C}$ (gradient $2.6{ }^{\circ} \mathrm{C} / \mathrm{min}$ ). In all cases, this was less than the specifications, which were $20 \mathrm{~min}$ for the duration, and $5{ }^{\circ} \mathrm{C} / \mathrm{min}$ for the gradient.

In all cases, the temperatures were above their minimum AFT. IRS was within its optimum thermal range for science. The focus mechanism may need to slow its speed below $-30{ }^{\circ} \mathrm{C}$. Note that, early in the project, the first cold case was run for six potential landing sites. Holden crater was found to be to most critical for survival, and from that moment on, all worst-cases simulations were run on that basis. The average power and energy for the survival heaters at Jezero crater are expected to be two-thirds of Holden's.

Hot cases (4 scenarios) verified that the temperatures of all sub-systems will stay within their maximum AFT, and thermal gradients will be compliant with design assumptions. The first two cases were non-operational; both are related to the Sun-safe idiosyncrasy of the design (see Sect. 4.10.2).

- In the first simulation, the RSM stayed pointed in one direction, and the Sun passed through the telescope field of view over the course of $80 \mathrm{~min}$. The secondary mirror was in its sun-safe position. It warmed to $20^{\circ} \mathrm{C}$, and the baffle around it to $35^{\circ} \mathrm{C}$. All other temperatures were below $10{ }^{\circ} \mathrm{C}$.

- The second case is highly unlikely: the telescope was tracking the Sun all day long, with the secondary mirror in a Sun safe position. The secondary mirror reached $66{ }^{\circ} \mathrm{C}$ and the baffle $83{ }^{\circ} \mathrm{C}$. These values have been taken into account in the qualification of these parts. In the model, the focus stage reached $46^{\circ} \mathrm{C}$, which was less than its bake-out temperature. All other temperatures were found to be within their AFT.

For the two other cases, the Mast Unit was on for $2 \mathrm{hr}$ at maximum power after $20 \mathrm{~min}$ of warmup. The primary goal was to test the power dissipation within the EBOX, and to make sure that all systems stay below their maximum AFT, and to identify when the IRS can operate at full performance.

- In the third simulation, the operations started at 11:00 local time. The telescope was pointing to the horizon. The warmups were not activated. All temperatures stayed within their AFT range, but the IR photodiodes were below $-5{ }^{\circ} \mathrm{C}$ (where performance is optimized) only from 11:00 to 12:10.

- The last simulation was similar to the previous one, but operations started at 13:00. All temperatures stayed below their maximum AFT and boards stayed below $70{ }^{\circ} \mathrm{C}$ (maximum temperature for part stress analysis). The IRS could not reach its best performance.

Again, the worst cases were conservative, and there is little or no chance that these cases occur on Mars. Their study has led to various changes in design, sub-systems qualification ranges and specific operational modes. In the end, a simplified 15-node model was built from these analysis. It was then rescaled to FM hardware and test results, with discrepancies $<10 \%$. For the record (an important input for the operations), the FM EBOX thermoswitches (two in series) for the survival heaters close at $-34.9^{\circ} \mathrm{C}$ and open at $-29.3{ }^{\circ} \mathrm{C}$. On the FM OBOX they close at $-36.3^{\circ} \mathrm{C}$ and open at $-29.5^{\circ} \mathrm{C}$.

\subsubsection{Thermal Tests and Validation}

At several points in the project, thermal tests were conducted at CNES (Toulouse, France) and at LESIA (Meudon, France) (Fig. 63). The three primary campaigns were for the STM, EQM, and FM. As the FM unit was evolving, the last test was done twice. 


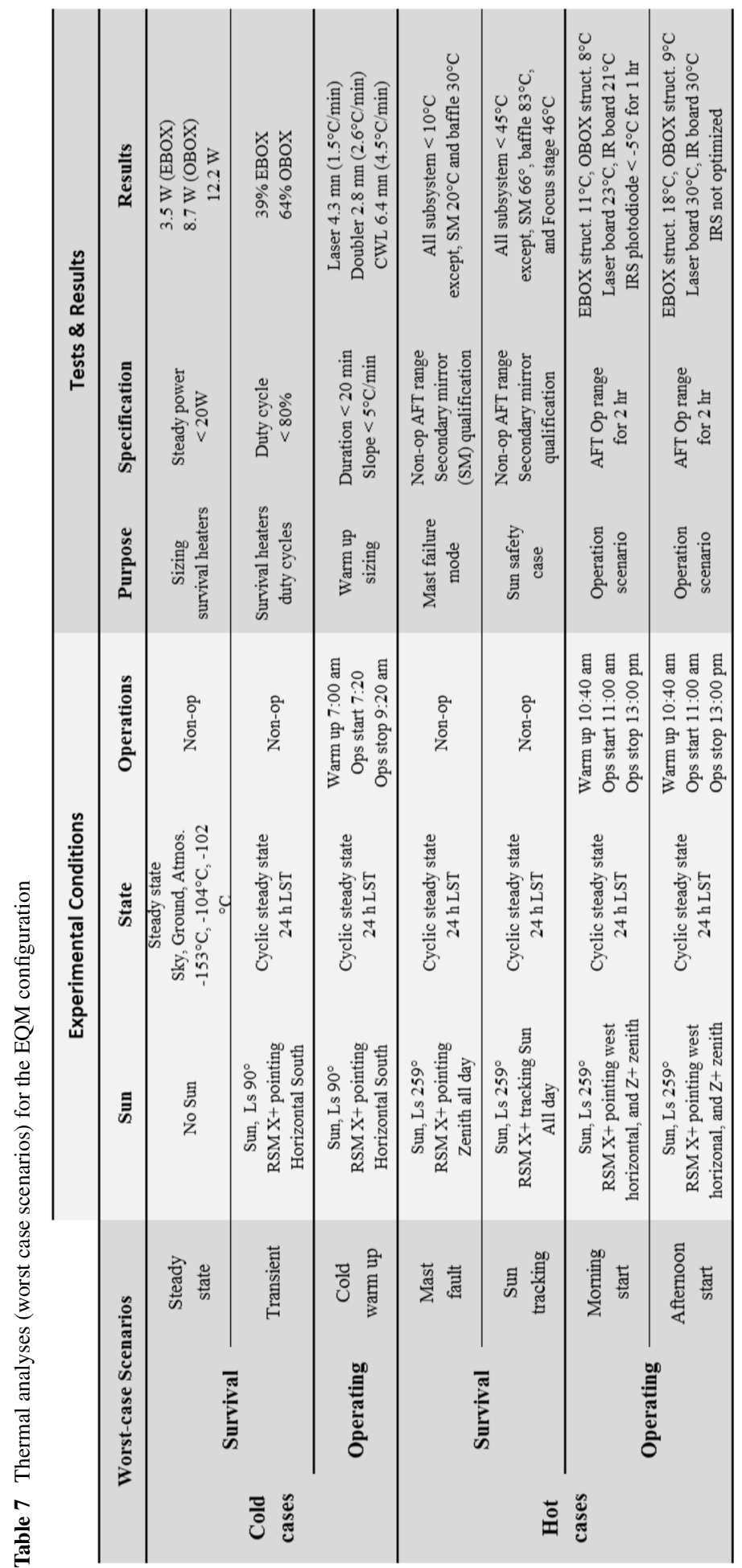




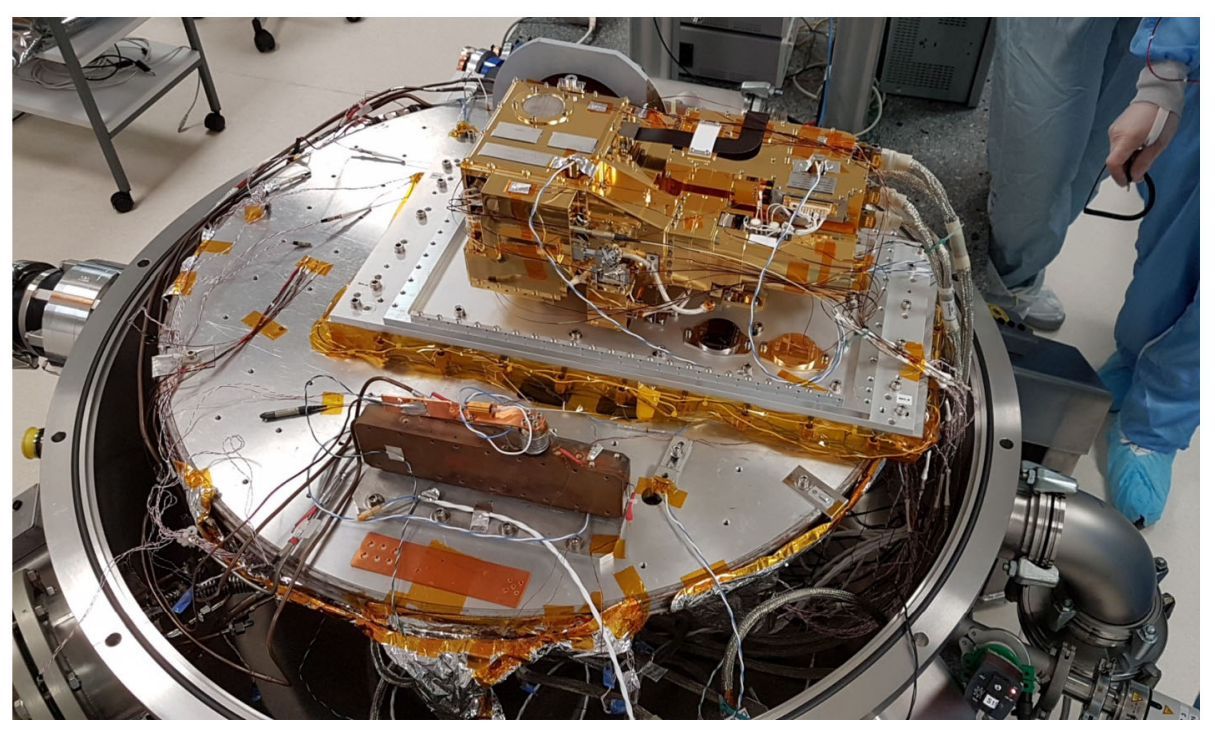

Fig. 63 Thermal tests at LESIA, Meudon. IR calibration of the flight unit

Early in the project (mid-2016), the STM was tested at qualification levels for three types of environments: Sun safety (7 Torr $\mathrm{CO}_{2}$, room temperature), Cruise (secondary vacuum, cycling temperatures), Mars (7 Torr $\mathrm{N}_{2}$ or $\mathrm{CO}_{2}$, cycling temperatures). The primary objective was to compare SuperCam's thermal status to ChemCam's, especially for issues such as Sun safety and regulation of survival heaters. It helped the team to prepare for qualification of the overall thermal architecture and provided inputs (thermal paths and loads) for the qualification of the subsystems.

The EQM model was subjected to a complete set of environmental tests (end of 2017) at qualification levels for the Cruise and Mars environments, operational and non-operational ranges. All functions were tested, verifying that all subsystems stay within their AFT range. Control loops and durations for the warmups and TEC cooling were determined, as well as transfer functions for the PRT. The global thermal balance was compared to the simulations (see Sect. 4.6.2).

On the FM, the first series of tests were performed over two weeks at acceptance level (end of 2018), confirming the results of the EQM. The test was done a second time at protoflight temperature levels, between $-50^{\circ} \mathrm{C}$ and $+50{ }^{\circ} \mathrm{C}$ (non-operational), $-50{ }^{\circ} \mathrm{C}$ and $+45{ }^{\circ} \mathrm{C}$ (operational), two cycles of each. All functions were tested, and the final performance with temperature was established.

\subsection{Electrical Design}

Most of SuperCam electronics are in a dedicated Electronic Box (EBOX). Still, some functions require proximity to the systems they drive, and for this reason, they are located in the Optical Box (OBOX). Mast-Unit electronics have to control several subsystems: the photodiode to check the (red) laser flux intensity, the $\mathrm{CW}$ laser diode and its associate photodiode for the CWL autofocus, the imager cube, the shutter, the focus mechanism, the microphone, the pulsed laser, and the IR spectrometer. The Mast-Unit electronics must generate housekeeping data on each subsystem. It also handles two communication protocols (High Speed 


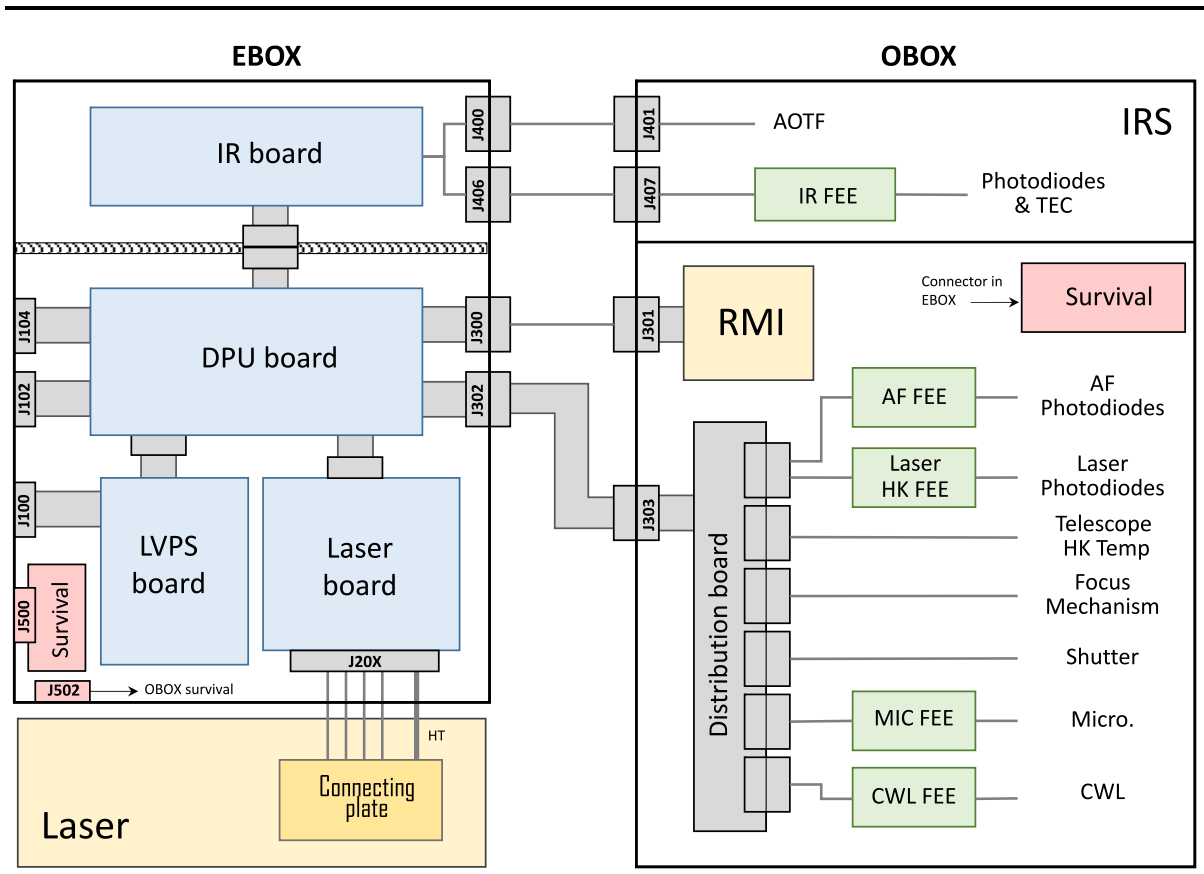

Fig. 64 Overall electronic architecture of the Mast Unit. Not all connector references are shown

Serial Link and Asynchronous Serial Link), receive commands, store and send data to and from the Body Unit.

The challenge of Mast-Unit electronics is to handle different classes of signals simultaneously:

- Two high voltage lines at $-1200 \mathrm{~V}$, which have to be switched in $10 \mathrm{~ns}$ to drive the laser Pockels cells;

- High current lines at 250 A during $200 \mu$ s in the laser diode stack;

- Radio Frequency (RF) signal from 33.1 MHz to 67.8 MHz;

- Low noise readout signals on the IRS photodiodes $(<0.1 \mathrm{pA})$ and microphone;

- $5 \mathrm{MHz}$ digital link to command the CMOS images, $25 \mathrm{MHz}$ data return rate.

Figure 64 summarizes the overall architecture. There are four separate boards located in the EBOX: the Low Voltage Power Supply (LVPS) board, the Digital Processing Unit (DPU) board, the laser board, and the IR board. The IR board is shielded from the other boards. There are several harnesses between the different units: a flex cable from the DPU to the OBOX, a round cable from the DPU to the RMI, a shielded cable (SSMA-type) from the IR board to the IRBOX, dedicated high-current, high-voltage harnesses to the laser connecting plate, and several harnesses for commands and HK retrieval. A distribution board inside the OBOX feeds a whole series of Front End Electronics boards and subsystems.

\subsubsection{LVPS Board}

The LVPS board provides the Mast-Unit subsystems with secondary voltages from the rover's unregulated $28 \mathrm{~V}$. These include several sources at each of $\pm 5 \mathrm{~V}$ and $+3.3 \mathrm{~V}$, plus $+20 \mathrm{~V},+30 \mathrm{~V}$, all with $\pm 5 \%$ accuracy, except for the $-5 \mathrm{~V}$ at $\pm 10 \%$. The input voltage 


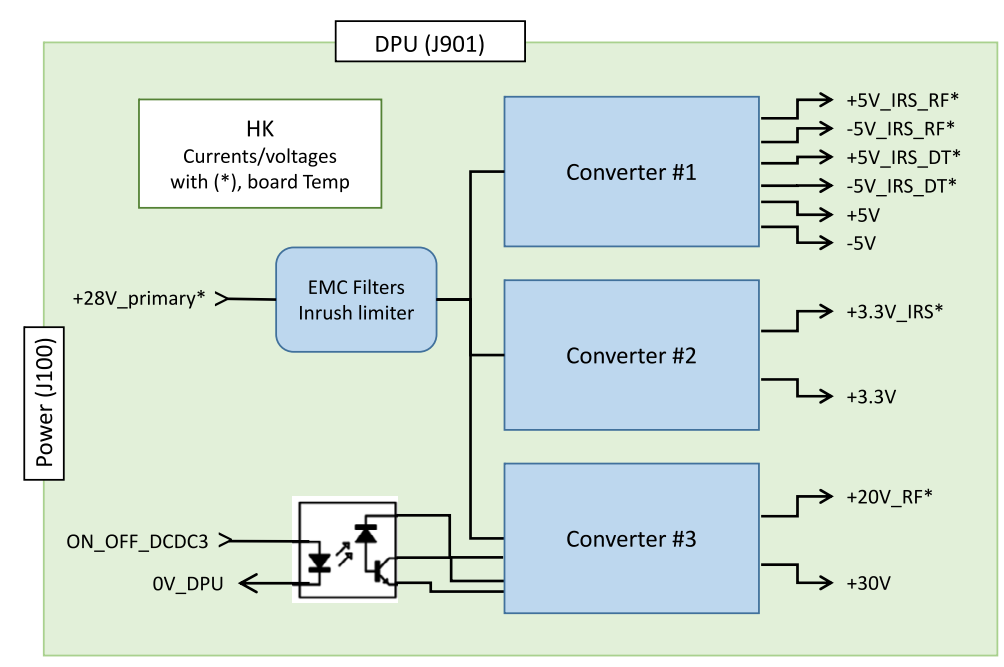

Fig. 65 The Low Voltage Power Supply (LVPS) board provides secondary voltages from the rover's unregulated $\sim 28 \mathrm{~V}$. There are three separate converter chains. The converter (\#3) that produces the $20 \mathrm{~V}$ RF and $30 \mathrm{~V}$ can be switched on or off by the DPU. The design includes an inrush limiter; the board also acquires various HK data
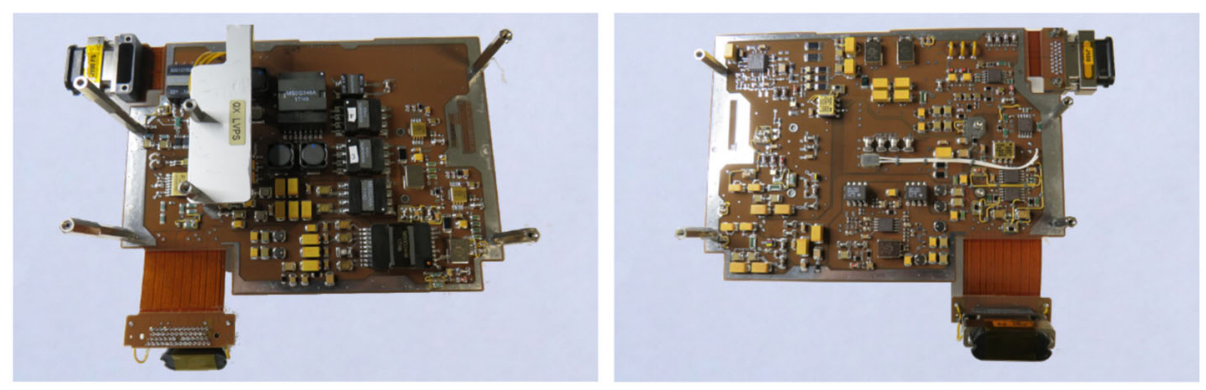

Fig. 66 LVPS board. (Left) Top side with transformers and filters for the converters and the inrush limiter. (Right) Bottom side with optocouplers and a PT1000 sensor

for the rover can range from $22 \mathrm{~V}$ to $36 \mathrm{~V}$. There are three separate converter chains: this is the minimum number of isolated converters needed to generate all output voltages and the maximum number of converters that can fit on the board (Figs. 65, 66). The converter that produces the $20 \mathrm{~V} \mathrm{RF}$ and $30 \mathrm{~V}$ can be switched on or off by the DPU. The Pulse Width Modulation's (PWM) controls of the converters are set to $500 \mathrm{kHz}$ for the $3.3 \mathrm{~V}$ and $5 \mathrm{~V}$ chains and $250 \mathrm{kHz}$ for the $20 \mathrm{~V} / 30 \mathrm{~V}$ chain to have a good efficiency and to facilitate postconverter filtering. An 8-channel multiplexer monitors the secondary voltages, the heatsink temperature, and the current on the input line. Note that $\pm 8.6 \mathrm{~V}$ are actually generated for the IRS detector, which are later converted to $\pm 5 \mathrm{~V}$ in the IR board by linear regulators. The design also includes an inrush limiter and an under-voltage lockout, and is tolerant of abrupt switch-off of the input line. There is an external connector to the Body Unit for power and an internal connector to the DPU board. Finally the board insulation between the rover $28 \mathrm{~V}$ and the chassis ranges from 20 to $100 \mathrm{MOhms}$. 


\subsubsection{DPU Board}

The DPU board is the centerpiece of the Mast-Unit electronic architecture. From a hardware point of view, its role is to:

- Switch on/off the 20 V/30 V converter on the LVPS board;

- Perform linear regulation and filtering for all voltages except those for the IRS;

- Supply power to all sub-systems;

- Control Front End Electronics (FEE) for each sub-system;

- Control the motor driver for autofocus;

- Communicate with the Body Unit (two protocols);

- Store the Boot Software, store the Application Flight Software;

- Store RMI images and microphone recordings;

- Monitor housekeeping data (temperature, voltages) using a slow 16-bit ADC;

- With a 12-bit ADC, to process the autofocus signals, to monitor the laser photodiode signal, to control laser shooting, and to record acoustic data.

The board is structured around a Field Programmable Gate Array (FPGA) that has an embedded 8051 soft microprocessor (Figs. 67, 68). For storage purposes, a 32 kBytes rad-hard One Time Programmable Memory (PROM) is implemented for the Boot Flight Software (BFSW). A $128 \mathrm{kB}$ nonvolatile Magnetic Random Access Memory (MRAM) is used for the Application Flight Software (AFSW), transfer function equations, RMI and IRS configuration tables, and parameter tables. A larger $32 \mathrm{M} \times 16$ bits Synchronous Dynamic Random Access Memory (SDRAM) stores RMI images and acoustic data. Finally, some internal memories to the FPGA are used to register special functions and off-core data memory. Unlike the BFSW, the AFSW is reprogrammable on Mars using a "patch" command. The Mast Unit can store up to three different codes for safety and redundancy.

The FPGA is a Microsemi RT3PE3000L FPGA with a CCGA484 package. This reprogrammable, nonvolatile, radiation-tolerant, flash-based FPGA is well-suited for SuperCam. The component is free of single event latch-up up to $68 \mathrm{MeV}-\mathrm{cm}^{2} / \mathrm{mg}$. Configuration cells stay protected from Single Event Upsets (SEUs) during flight. However, D-type flip-flops (DFF) and Synchronous Dynamic Random Access Memory (SDRAM) memories are sensitive to SEUs; the clocks and I/O banks are vulnerable to single-event transients. Thus, all DFF's are triplicated using the Synplify triple-module-redundancy option. For the SRAMs, clocks, and I/O, a hardware-software interaction analysis has been performed: a nondestructive failure rate - without mitigation - of about one event for the nominal mission was found and accepted. The flight design uses $\sim 90 \%$ of the $75 \mathrm{k}$ gates after triplication, $37 \%$ of Block RAM that are available in the FPGA, and I/O's account for $57 \%$ the resources.

A crystal oscillator generates a clock signal at $20 \mathrm{MHz}$ with $\pm 50 \mathrm{ppm}$ stability. It feeds the 8051 microcontroller and all drivers, using asserted signals to create lower clocks for each device. Another $25 \mathrm{MHz}$ clock is used to transfer the pixels from the RMI to the DPU board at $50 \mathrm{Mb} / \mathrm{s}$ using dual data rate technics. MOSFETs are used to switch on/off power for sub-systems. Hence, the microphone circuit can be turned on when needed. The same applies for the RMI cube, the CWL heaters, and the shutter. A full-bridge driver controls the current in the windings of the stepper motor. It can be disabled too. Associated with the motor driver, the limit-switch statuses are monitored continuously. The CWL diode control electronics and associated photodiode variable gain amplifier are also implemented on this board.

The DPU board is a 16-layer PCB with the FPGA on one side, and the motor driver on the other side. The DPU board has seven Micro-D connectors to the Body Unit (J102), the 


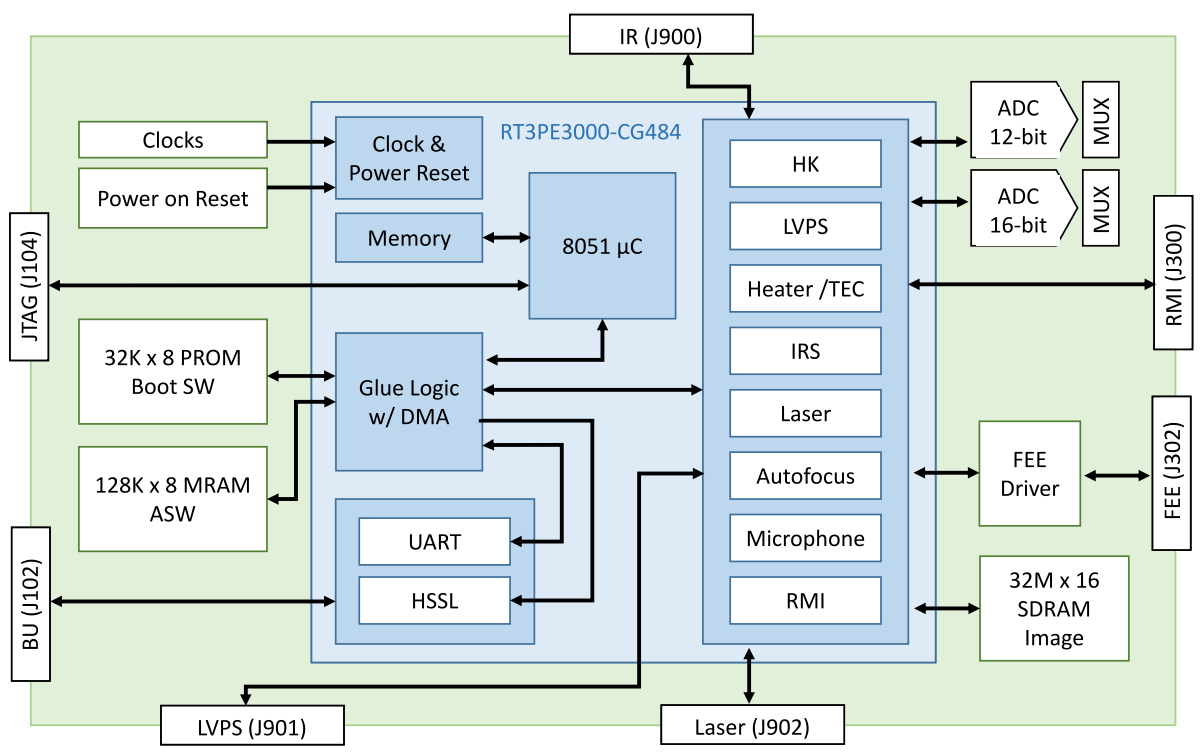

Fig. 67 The Digital Processor Unit (DPU) board is structured around the Microsemi RT3PE3000L FPGA, that has an embedded 8051 soft microprocessor and several drivers for the subsystems. A crystal oscillator generates a clock signal at $20 \mathrm{MHz}$. One-time Programmable Memory (PROM) is implemented for the Boot Flight Software; Magnetic Random-Access Memory (MRAM) is used for the Application Flight Software and various parameters. A larger Synchronous Dynamic Random-Access Memory (SDRAM) stores RMI images and acoustic data
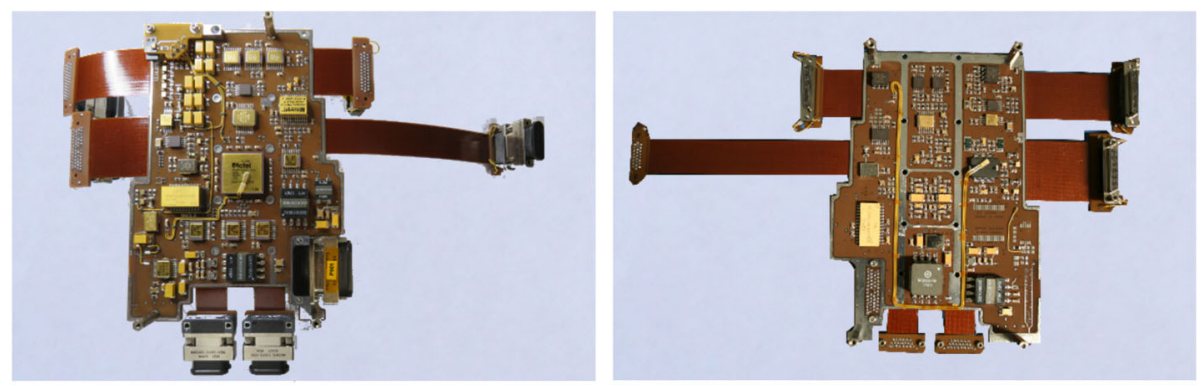

Fig. 68 DPU board. (Left) Top side with FPGA, with its thermal sensor, memories and ADC. (Right) Bottom side with motor driver, with its thermal sensor, and analog parts

LVPS board (P901), the laser board (J902), the IRS board (J900), the RMI board (J300), and the FEE board (J302). The test connector (J104) is used to download software for the debug and test modes, to disable the CWL and pulsed laser for safety.

\subsubsection{Laser Board}

The laser board is connected to the DPU board and directly to the laser (Figs. 69, 70). It stores energy for each laser shot, provides a high-current pulse (max. $250 \mathrm{~A}$ ) to the laser stack, and triggers one or two high voltage (max. negative $1200 \mathrm{~V}$ ) Pockels cells. 


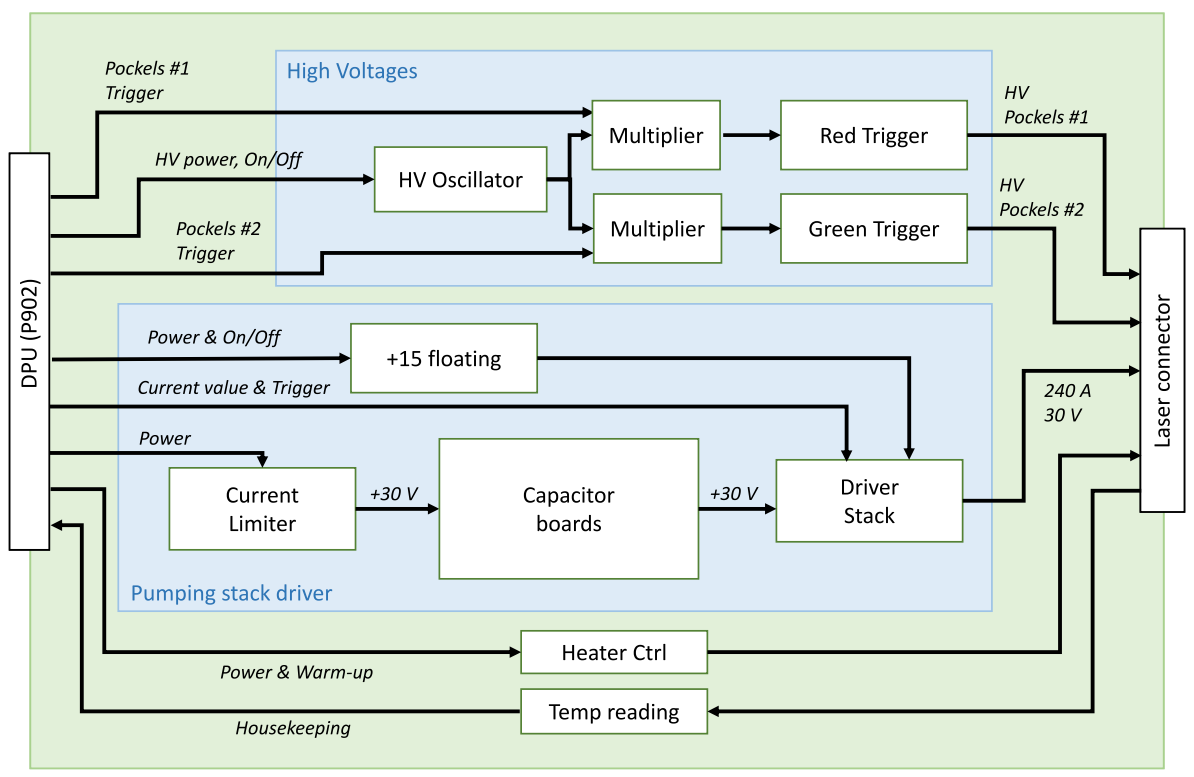

Fig. 69 The laser board stores energy for each laser shot into 350 solid Ta capacitors, and provides a highcurrent pulse to the laser stack. It triggers one high-voltage Pockels cell to generate the red beam, or two Pockels simultaneously for the green beam. The board acquires various HK data
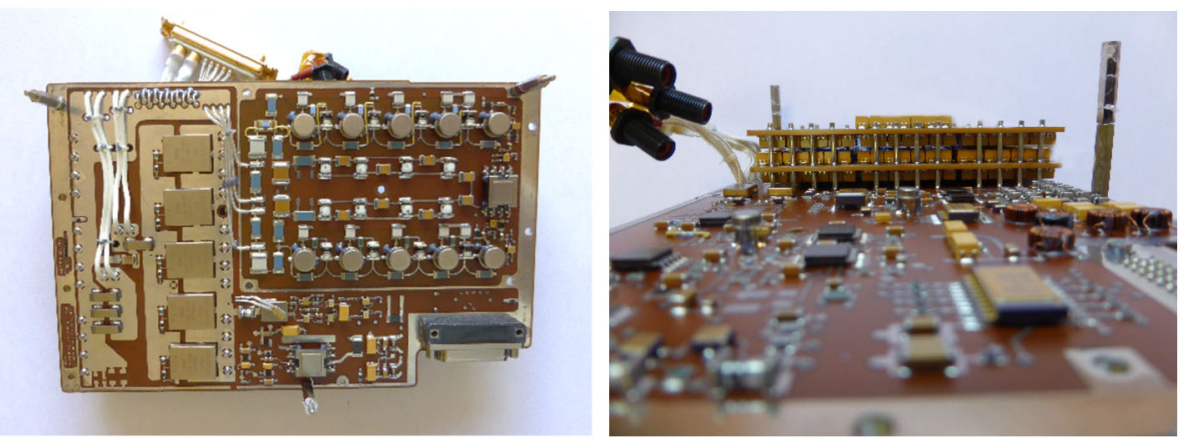

Fig. 70 Laser board. (Left) Top side with the two HV drivers, before closure of the EMC shield. (Right) Bottom side with the stack of 350 capacitors

To pump the laser stack, the laser board delivers an adjustable 40-250 A current pulse for $220 \mu \mathrm{s}$, with a standard deviation $<3 \%$, rise and fall times $\sim 20 \mu \mathrm{s}$. To store the energy, as for ChemCam, we prefer to rely on tantalum capacitors since they have a high energy storage capability compared to other capacitors that are available for flight. We use 350 solid $470 \mu \mathrm{F} 10$ VDC capacitors from AVX. They are distributed in 10 blocks of seven strings in parallel, five capacitors per string in series. Within a block, two failures in short circuits are acceptable. In the end, the pulse current per capacitor is $3.57 \mathrm{~A}$ (rated surge current $10 \mathrm{~A}$ ), the voltage is $6 \mathrm{~V}$ (rated voltage $10 \mathrm{~V}$ ). The circuit to generate the current step relies on five power $\mathrm{N}$ channel MOSFETs that are mounted close to the laser stack. In this configuration, 


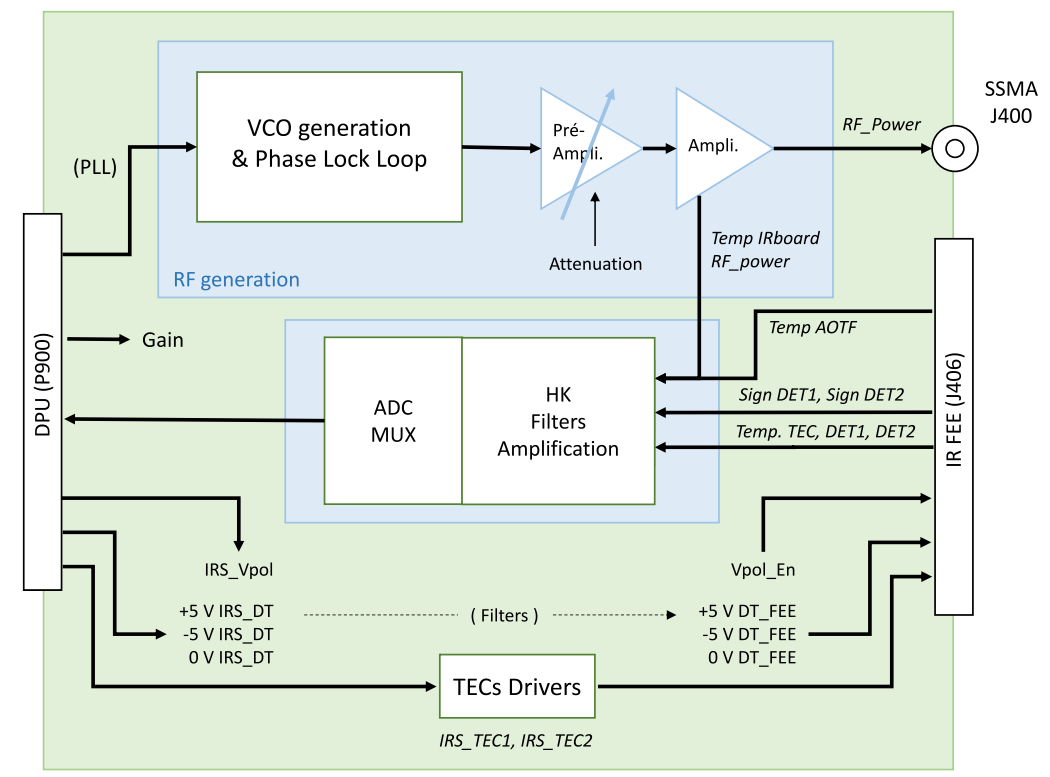

Fig. 71 The IR Board generates the Radio Frequencies (RF) that excite the AOTF filter, acquires data from the two photodiodes, drives TECs to cool the photodiodes, and acquires HK data. Dedicated voltage-filtered lines are used for RF functions, for the signal acquisition, and for the TEC regulation. The temperature of the detector is used for closed-loop control of the photodiode temperature

the voltage on the grid of the MOSFETs must be referenced to a local floating ground to avoid unintentional firing from spurious glitches on the general secondary ground.

There are two Pockels cells, one for the Q-switch and one for the doubler. Thus, the board generates two identical HV signals. When the green beam is needed, the doubler is triggered $50 \mathrm{~ns}$ before the Q-switch. The Q-switch signal is one microsecond long, negative $\sim 1200 \mathrm{~V}$ maximum in amplitude, and must fall within ten nanoseconds (see Wiens et al., this journal). The Q-switch voltage is generated from the $30 \mathrm{~V}$ line. There are independent voltage multipliers to avoid crosstalk when triggering the Pockels. The frequency of the freerunning oscillation of the circuit is $\sim 100 \mathrm{kHz}$. Several adjustments with five MOSFETs were necessary to obtain the required fall time, limiting transients on the components.

The Pockels cells voltages are adjusted to each laser. The range for pumping current depends on temperature (Sect. 4.3.1). The maximum value on Mars will never exceed $170 \mathrm{~A}$, giving us sufficient margins on the board design. The board acquires housekeeping data, including four temperatures inside the laser cavity. Both the laser stack and the doubler have heaters that are supplied by the secondary $30 \mathrm{~V}$. The frequency of the pulse width modulation is $9.79 \mathrm{kHz}$ to avoid perturbation with the microphone when the lase fires (see Sect. 4.3.6).

\subsubsection{IR Board and Front-End-Electronic}

The IR Board generates the Radio Frequencies (RF) that excite the AOTF filter, acquires data from the two photodiodes (one at a time, cold redundancy), drives TECs to cool the photodiodes, and acquires HK data. The acquisition function is physically separated into 1) pre-amplification of the signal on the small IR FEE inside the spectrometer itself (IRbox), and 2) signal processing and digital conversion on the IR board (Fig. 71). 

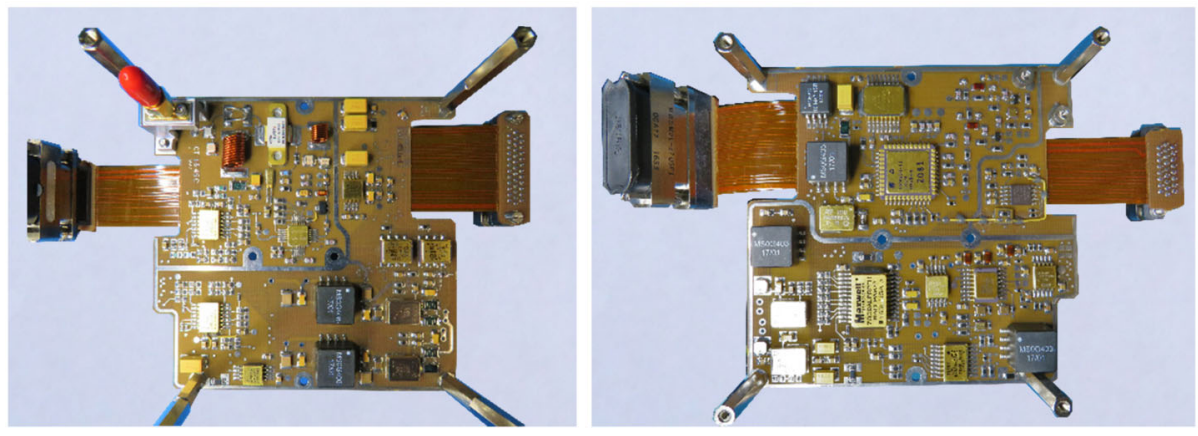

Fig. 72 IRS board. (Left) Top side with the RF power amplifier and SSMA connector. (Right) Bottom side with RF generation in the upper part, and detectors reading electronics on the lower part

The IR board connects to the DPU board and the IR FEE board (Figs. 64, 71, and 72). Note the SSMA connector (J400) between the board and the IRbox for the RF signal to the AOTF; it is mounted on a dedicated fixture with a short cable (Fig. 72). More than on any other boards, special attention has been given to grounding and filtering. Dedicated voltages are used: $\pm 5 \mathrm{~V} \_I R S \_R F$ and $+20 \mathrm{~V} \_I R S \_R F$ for RF functions, $\pm 5 \mathrm{~V} \_I R S \_D T$ for signal acquisition, +3.3V_IRS for the TEC regulation. These voltages are filtered by common mode and differential filters. Optocouplers were inserted in the command path between the DPU and IR FEE inside the IRbox itself. Partition walls for EMC shielding between the different IR board functions were integrated into the EBOX structure and cover, to separate the RF generation from the signal acquisition. To prevent radiated emissions, for electromagnetic compatibility, an electromagnetic interference gasket is used to seal the gap between the SSMA fixture and the IR Board lid.

The RF generation circuit locks the Voltage Controlled Oscillators (VCO) at a selected frequency between $33.1 \mathrm{MHz}$ to $67.8 \mathrm{MHz}$. A controllable gain controls the first stage of amplification. The VCO can be disabled to switch the RF signal off. The performance of the clock oscillator allows the stability of the RF signal to be better than $\pm 10 \mathrm{kHz}$ in frequency (including temperature, voltage, and aging drift).

The acquisition circuit acquires the photodiode signals and various HK data, including the IR Board temperature, AOTF, Detector (MCT chip) and detector bracket temperatures, and the RF power signal. The temperature of the detector is used for closed-loop control of the photodiode temperature. The measurement of the photodiode signal is the most critical acquisition, as the main contribution to this signal is the dark current (Poisson statistics), which can be seen as an offset. The colder the photodiode, the lower the dark current. The optimal temperature of the photodiode ranges from $-60{ }^{\circ} \mathrm{C}$ to $-90^{\circ} \mathrm{C}$. At $-60{ }^{\circ} \mathrm{C}$, this dark current is around $40 \mathrm{nA}$ and at $-90^{\circ} \mathrm{C}$, about $2 \mathrm{nA}$. In comparison, the IR flux from Mars is expected to be between $10 \mathrm{pA}$ and $1 \mathrm{nA}$. As the lowest temperature of the instrument is $-37^{\circ} \mathrm{C}$, switching on the TEC before using the photodiode is required. As an aside, we see that the readout noise on the IR board cannot be greater than $0.1 \mathrm{pA}$.

To measure this flux, we perform two consecutive measurements with the AOTF successively on and off. The difference represents the diffracted IR flux and some variations of the thermal background. The two acquisitions must be performed as close as possible to each other to limit these variations, so both are measured for each spectel before moving to the next one. Both acquisitions can also be repeated up to 128 times for the same spectel, with coherent addition to reduce the noise level and to increase the SNR. 


\subsubsection{OBOX Electronics}

Even if it is not optimum to mix electronics with optics for contamination control, a few proximity boards must be placed next to their active sensors. Hence there are FrontEnd-Electronics (FEEs) for the IRS photodiodes (see Sect. 4.3.3), the microphone (see Sect. 4.3.6), and three additional ones that are presented below: the laser energy-monitoring FEE, the continuous wavelength laser FEE, and the associated autofocus FEE. There is also a distribution board in the OBOX, and a small PCB next to the focus table.

The DPU monitors the red laser beam energy during LIBS shots. The FEE consists of a photodiode and a trans-impedance amplifier with peak detection. The space-qualified photodiode has a high parasitic capacitance (S1336-8BK by Hamamatsu Photonics). A fraction of the beam energy is collected by the photodiode through a diaphragm. The challenge is to co-design the diaphragm with the FEE in order to match their respective dynamic range.

The CWL beam is collimated by an aspherical lens and co-aligned with the telescope boresight. The maximum output power is $50 \mathrm{~mW}$, and the signal is modulated at $10 \mathrm{kHz}$. This diode laser is a commercial (COTS) device from JDSU. A batch of 100 laser diodes was procured and went through lot acceptance tests by CNES. The DPU board implements the CWL driver, but to reach good stability of emission, a FEE board was added next to the CWL itself. It integrates the output power of the CWL, and then adjusts the CWL current bias to stabilize its optical power; i.e. its runs in a fixed-power mode. This design can only increase the pump current (but not decrease it) therefore the system baseline was trimmed below the minimum required bias, so at least a small compensation is always added, even at the nominal temperature $\left(-10^{\circ} \mathrm{C}\right)$.

The AF photodiode (same S1336-8BK) integrates the CWL light reflected by the target. The FEE consists of a trans-impedance amplifier, followed by another amplifier stage to monitor the $\mathrm{CW}$ light reflected by the target.

\subsection{FPGA and Flight Software}

The Mast-Unit logic is spread over hardware and software supported by a single FPGA. The flight software, composed of the BFSW and AFSW, runs on a R8051XC2 microprocessor implemented into the FPGA. The role of the software is to interpret commands, sequence operations, control and monitor subsystems, and to perform scientific algorithms (CWL and RMI autofocus, RMI auto-exposure, HDR imaging, and IRS measurement). For that, the flight software benefits from custom hardware support/acceleration implemented in logic in the rest of the FPGA (dedicated drivers).

\subsubsection{Communication with the Body Unit}

All commands from the Body Unit use the Universal Asynchronous Receiver-Transmitter (UART) link at 9600 baud with bytes coded in big-endian. The baud rate is reconfigurable in flight, up to 115200 baud. Basically, the Mast Unit processes the command, verifying the conformity and sending an acknowledgment within a few milliseconds. If an action is required, the Mast Unit sends an acknowledgment, followed by the data. In case of a nonconformity, malfunction or non-convergence of the algorithms, the Mast-Unit acknowledgment or data reply includes a specific code that can be interpreted by the Body Unit. In case of timeout (e.g. failing to communicate with the RMI), the Mast Unit has fault protection mechanism, and at a higher level, the Body Unit can repeat or stop the activity. 
A second communication path to the Body Unit is the High Speed Serial Link (HSSL) protocol. The default speed is $10 \mathrm{Mbit} / \mathrm{s}$. It can be lowered to $1 \mathrm{Mbit} / \mathrm{s}$. This link is used to download data from the DPU SDRAM memory, either image or sound. At this speed, three Low Voltage Differential signal (LVDS) lines are used: one frame synch line, clock, and data on clock rising edge.

The last communication path between the Body Unit and Mast Unit consists of LVDS discrete signals: the first one is the Mast-Unit "Reset" signal being active high with $1 \mathrm{~ms}$ duration (equivalent to power-on-reset); the second one is the "Fire Laser" signal triggering each laser shot; the last one is the signal going down to synchronize the transmission spectrometer shutter (Wiens et al., this journal).

\subsubsection{Monitoring of State-of-Health Data}

The Mast Unit monitors more than 29 temperatures, voltages, or currents, and about 24 digital HK channels to reflect the status of the sub-systems. Most HK data are sampled by a 16-bit ADC every $67 \mathrm{~ms}$. One hundred twenty-eight values are averaged together. HK values are transmitted to the Body Unit. It checks that the Mast Unit is within its AFT (if not, it sets an error status flag). The Body Unit can also change some command parameters, depending on the OBOX temperature, such as the motor speed or laser pumping current. Finally, the Body Unit will not allow the CWL to turn on if the warm up is not "ready", and will not allow IRS acquisition if the TEC is not on. A different 12-bit high-speed ADC is used to acquire event-triggered HK's, such as pumping current, stack voltage, and optical power during laser shots, or CWL optical power during the autofocus procedure, or microphone data during sound recording. When the IR spectrometer is operated, a dedicated 16-bit ADC on the IR board records the AOTF and TEC temperatures.

\subsubsection{Thermal Control}

Thermal control is implemented for the pulsed laser stack and doubler: they need to be warmed up to $-15^{\circ} \mathrm{C}$ and $-10{ }^{\circ} \mathrm{C}$, respectively, for better performance. The CWL needs to warm up to $-10{ }^{\circ} \mathrm{C}$ to avoid condensation of residual humidity trapped in the diode cavity. The infrared photodiodes need to be cooled to $<-60{ }^{\circ} \mathrm{C}$ to reduce the noise. For the warm-ups, a standard Proportional Integral Derivative (PID) control is implemented, with derivative value set to 0 , coupled with a ramp control to reach the set point smoothly. We limit the slope to $5{ }^{\circ} \mathrm{C} / \mathrm{min}$ to avoid stressing the laser and CWL. A PID and a controlled ramp are also used to cool down the IRS photodiodes. The cooldown is also controlled so that the maximum temperature rate of change recommended by the manufacturer is not exceeded. These thermal controls are fully parameterized in the FPGA registers and they can be updated on Mars.

\subsubsection{Dedicated Drivers}

The FPGA implements Very High Speed Integrated Circuit Hardware Description Language (VHDL) drivers to control various subsystems, such as the laser, CWL autofocus, microphone, IRS, RMI, and SDRAM. Each is described briefly below.

Once it receives a "Fire-the-Laser" signal on the dedicated line, the laser driver operates a few checks: a) the signal must be received for at least $200 \mu$ s to avoid firing on a spurious signal, b) the maximum number of shots must not been reached, and c) the burst frequency is within acceptable range. Then it generates various signals to control the pumping current, 
the current duration, and the Pockels delay. For a green beam, the shutter is activated before the shots. If LIBS and microphone are activated simultaneously, the laser driver triggers the microphone driver. Last, the Mast Unit sends a "laser-sync" signal on a discrete line to the Body Unit, which starts the delay timer for the intensifier, opening the gate by pulsing the cathode voltage at the proper time.

The Autofocus driver generates a $10 \mathrm{kHz}$ signal to modulate the CWL beam intensity and the demodulation of the photodiode is done digitally by mathematical processing. It drives the L6258 motor controller and monitors the status of the limit switches to prevent any movement of the motor when they have been triggered. The synchronous detection is the basis of the CWL-AF. The analysis of these data is given in Sect. 4.9.1.

The Microphone driver interfaces with the HK driver which drives the high-speed ADC (sampling frequency up to $100 \mathrm{kHz}$ ) and stores data in the SDRAM memory bank. This driver is also called by the laser driver to synchronize the recording to the LIBS shots (continuous or pulse mode, see Sect. 4.3.6).

The IRS driver handles the generation of RF signal for Phase-Lock Loop (PLL) control, power control, signals for housekeeping with a dedicated 16 bit ADC and the main timing generation for each pixel, including configuration of PLL, integration, and a wait cycle for each dark and signal.

The RMI cube is configured using serial peripheral interface (SPI) protocol to set various parameters (e.g. exposure time). The image is transferred from the RMI to the DPU FPGA using two LVDS lines at $50 \mathrm{Mb} / \mathrm{s}$ in dual data mode. Specific clock-domain crossing techniques using dual-port RAM have been implemented in the FPGA to capture the pixels with the $25 \mathrm{MHz}$ RMI clock, and to store the pixels in the SDRAM with the $20 \mathrm{MHz}$ master clock.

A complex state machine is responsible for controlling the various SDRAM modes, including refreshing, basic writing, basic reading, burst writing, burst reading, etc., depending on the actual Mast-Unit operations, sound acquisition, image acquisition, HDR image processing, or RMI autofocus. The SDRAM can store up to eight complete RMI images or eight sound recordings of 42 second at $100 \mathrm{kHz}$. It is organized as 64 banks of one MB each.

\subsubsection{BFSW-Boot Flight Software}

The BFSW automatically loaded at power-on (Fig. 73) allows the AFSW and Firmware version numbers to be checked. Most importantly, it can also patch or dump the AFSW. The "patch" command loads a new AFSW or new parameter tables into the memory, while the "dump" checks the memory content. The "BIST" (Built-In Self Test) command needs to run before the start of AFSW to check the integrity of the memory content. If an error occurs, the Body-Unit software halts the MU boot and sends an error code to the rover. Otherwise, the "jump" command launches the application software.

\subsubsection{AFSW - Application Flight Software}

Three versions of the AFSW are loaded into the memory, the last two versions developed before launch, and a debug mode specific version (only useful on Earth with a specific plug). The AFSW implements all commands necessary to control and monitor the Mast Unit, from simple commands (switch on/off) to complex algorithms (for AF, HDR) and combined sequences (LIBS and MIC modes). The commands are organized in four groups (pink circles in Fig. 73): i) On-off basic commands (e.g. Turn_RMI_on); ii) Subsystem configuration (e.g. 


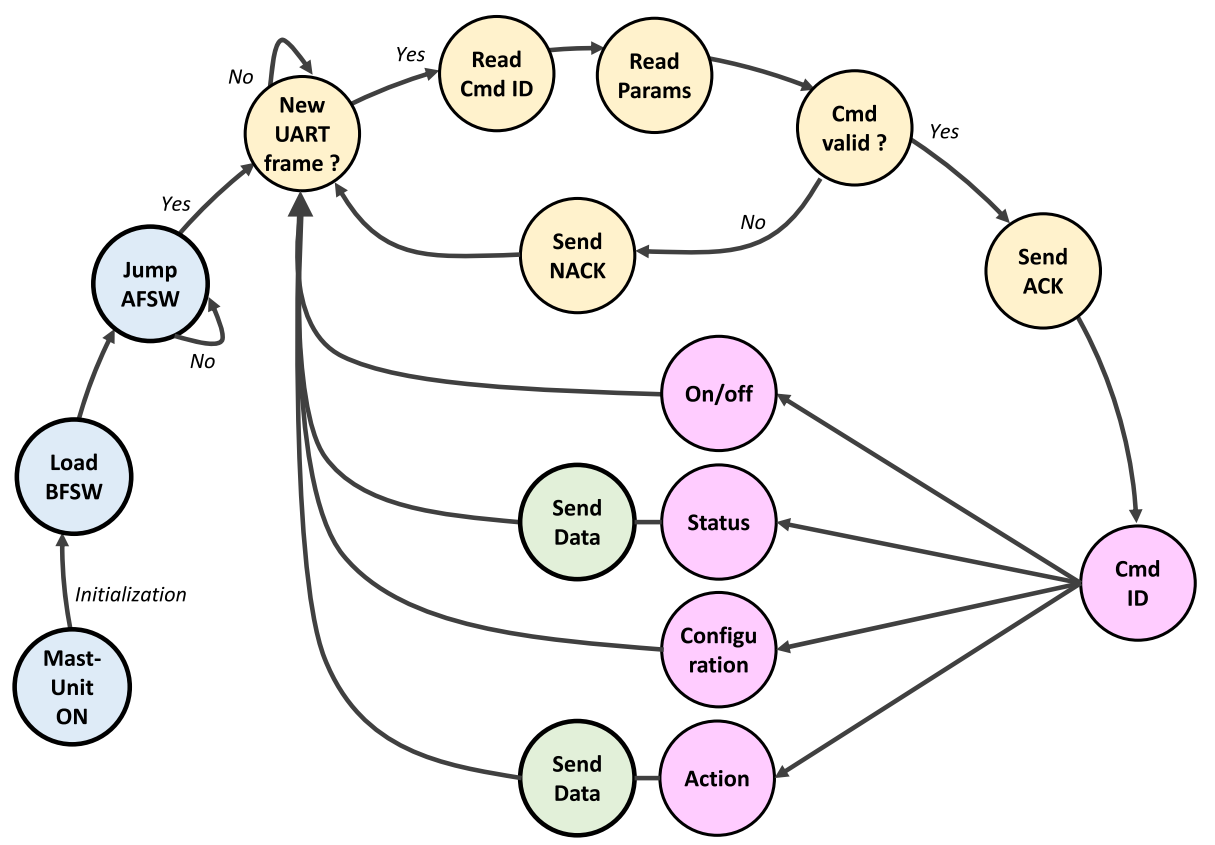

Fig. 73 Boot Flight Software (BFSW) and Application Flight Software (AFSW) simplified state diagrams. Other acronyms are: ACK, positive acknowledgement, NACK, negative acknowledgement, Cmd ID, command identifier

Configure_number of displacements, steps, and backlash parameters for autofocus); iii) Actions (e.g. Do_Autofocus); iv) Status (e.g. Send_Housekeeping). For groups iii) and iv) (except No-Op, Test_high_speed_link, and Abort MU), data replies are sent back after the command is executed, and the Body Unit handles the information. If the information does not correspond to what was expected, the Body Unit decides to go on or stop operations, depending on the specific command and the information received. In any case, the overall state of health of the Mast Unit can be retrieved using the "send housekeeping" command, which provides 29 analog values (temperature, voltages, and currents) and 24 digital values (thermal controls, on/off, and limit switch status).

\subsubsection{Software Development}

Software was developed using the Keil $\mu$ Vision development environment, with the CX51 compiler and the LX51 linker. European space software standards (ECSS E40, ECSS Q80) were tailored to be compatible with the organization means and to focus on important activities. Regarding code quality, MISRA coding rules were applied and the code was fully verified against runtime errors. For that, CNES was in charge of an independent verification using the Polyspace Code Prover R2014 tool provided by MathWorks. In the end, the BFSW and AFSW codes were delivered in a HEX format, and loaded into the Mast-Unit memories.

The BFSW and AFSW were tested extensively before their delivery. The first level of testing was performed at the hardware/firmware and software level using ModelSim, and a dedicated EGSE GUI. The FPGA drivers (IP 8051 and specific designed drivers) and also the surrounding components (such as memories, ADC and CMOS sensor) were modeled 
and implemented in the simulation tool, and run in a real-like environment thanks to test patterns generated by the EGSE. Then, validation tests have been defined and executed on a specific EGSE built around a prototype DPU. Code coverage measurement has been done so as to verify that all decisions (for the BFSW) and instructions (for the AFSW) have been tested at least one time. Test results were automatically analyzed at EGSE level. In case of not-covered decisions or instructions by validation tests, specific verifications have been done or unit tests have been defined and executed directly on the target, or in simulation mode using the simulator provided with the Keil $\mu$ Vision development environment. This technical solution has also been used during the development for testing specific scientific algorithms. In this case the results were compared with expected results computed using Matlab.

Tests were performed at room temperature, and occasionally at low or high temperature. Before delivery, more than 300,000 commands were sent over 142 hours in various combinations of hot, cold, vacuum or low-pressure nitrogen environments.

\subsection{Scientific Algorithms}

The Mast Unit holds several algorithms for RMI autoexposure, piecewise and multi-frame HDR modes, and RMI and CWL autofocus. Autoexposure and autofocus are absolutely critical to the success of the investigation.

\subsubsection{Autoexposure}

The auto-exposure algorithm avoids the saturation of images and determines the optimum exposure time. It starts from an exposure seed given from the ground. The auto-exposure algorithm stops as soon as maximum and minimum thresholds are met, over a specified number of pixels inside a region of interest. It is based on three possible multiplications or divisions with successive factors equal to $3,3^{0.5}$, and $3^{0.25}$. In total, 15 exposure times are possible, ranging from 0.15 to 6.84 times the seed time. The thresholding of bright or dark pixels is performed by the Mast Unit on the fly during the image transfer from the RMI to the SDRAM. All these combinations were tested, and a procedure was developed to find the appropriate parameters. The goal was to strictly avoid saturated images and to slightly relax the constraints on underexposed images to converge quickly.

\subsubsection{HDR Modes}

CMOS sensors have naturally shallow wells (13.5 $\mathrm{ke}^{-}$for the RMI) compared to CCD's (220 $\mathrm{ke}^{-}$for the Thomson TH7888A on ChemCam; Maurice et al. 2012). To cope with this limitation and regain some dynamic range, especially when a fraction of the field of view is in shadow, we have implemented two High Dynamic Range (HDR) techniques: the piecewise and multi-frame modes.

The multi-frame HDR mode is run outside the CMOS. Several images are taken at the same focus with different exposure times. An image is built from the sum of the different images. Prior to the sum, pixels above a programmable threshold are clipped to maintain a good linearity. Up to 7 images can be added. Parameters are the exposure time of the first image, which can also be obtained by auto-exposure, the clipping threshold, and the series of exposure increments between the images. The ground processing will reconstruct the full linearity by weighting the pixel value according to its corresponding image. The obtained linearity is the same as it should be if the sensor was perfectly linear with exposure time on 
the full dynamic range of all images. It is therefore important to (1) define the clipping value to stay within the linear zone of the sensor, (2) to know the number of images summed and their respective exposure times, and (3) to choose the starting exposure time not to saturate any pixel in the first image.

The piecewise mode was built into the CMOS image sensor. Illuminated pixels which reach a programmable voltage are clipped while leaving the darker pixels untouched. The clipping level can be adjusted twice within one exposure time to achieve a maximum of three slopes in the pixel response curve. If the knee points and slopes are well chosen, this should guarantee that at the end of the exposure, the brightest pixels are not saturated. This mode is commanded through the table of registers loaded to the CMOS detector, specifying two voltages and three exposure times. The voltages control the position of the knee points while the exposure times control the slopes. This mode is not the default mode, since it introduces a non-linear response. However, it is a backup to the multi-frame mode, especially when linearity is not an issue, while performing RMI autofocus, for instance.

\subsubsection{Autofocus}

We implemented two methods to focus the telescope, both of which have been tested on ChemCam, but in the case of ChemCam, the calculations are done in the Body Unit while they are performed by the Mast-Unit FSW for SuperCam. Each method starts from a seed distance given by the rover Navcams, or estimated by the operators on the ground (see Sect. 3.4).

The "RMI autofocus" became the new focusing method on ChemCam after Sol 1000 (Peret et al. 2016). It does not require any dedicated hardware but relies on the existing RMI capability. The algorithm starts from several images at different positions of the secondary mirror. For each image, the FSW calculates a Laplacian score for each green pixel $(i, j)$ from neighboring pixels, with spacing to account for the instrument PSF:

$$
L_{i, j}=4 P_{i, j}-P_{i+2, j}-P_{i-2, j}-P_{i, j+2}-P_{i, j-2}
$$

The final score per image is the sum of absolute values of $L_{i, j}$ over the chosen region of interest (ROI). From the three scores around the highest value, the algorithm interpolates a parabola and computes its vertex. If the highest score is at the start or at the end of the scan, no interpolation is performed. A few parameters are adjustable: the size of the ROI, its Y-position on the detector, the (odd) number of images, and their separation in motor steps. Typically, 15 images separated by 11 steps are recorded. The absolute value of the Laplacian score depends on the size of the ROI and the small-scale details of the image itself; the ROI is typically $140 \times 140$ pixels (i.e. $70 \times 70$ green pixels). Figure 74 (bottom) shows RMI AF curves from the same target at four distances. The dynamic range of the signal is very large but the extent in steps is small. This configuration runs in $\sim 90 \mathrm{~s}$ at all distances. Autoexposure or manual mode can be used for the first frame; all subsequent images use the same exposure time. In the end, only the best-focus image will be returned to the ground.

Using the RMI autofocus, if we assume a precision equal to half the distance between two consecutive frames ( 5 steps), at $2 \mathrm{~m}$ distance the algorithm finds the best focus within $\pm 0.1 \%$, at $4 \mathrm{~m}$ it is within $\pm 0.2 \%$, and at $7 \mathrm{~m}$ it is within $\pm 0.33 \%$ of the distance. This precision is within the DOF of the RMI (see Fig. 52). Therefore this algorithm meets its goal to find the best focused image, at least in the ROI area which is chosen to overlap with the other techniques (LIBS, TRR/L, and VISIR).

The "CWL autofocus" was the baseline technique on ChemCam before it failed on Sol 800. Like a laser pointer, a Continuous Wavelength Laser (CWL) emits a collimated beam 


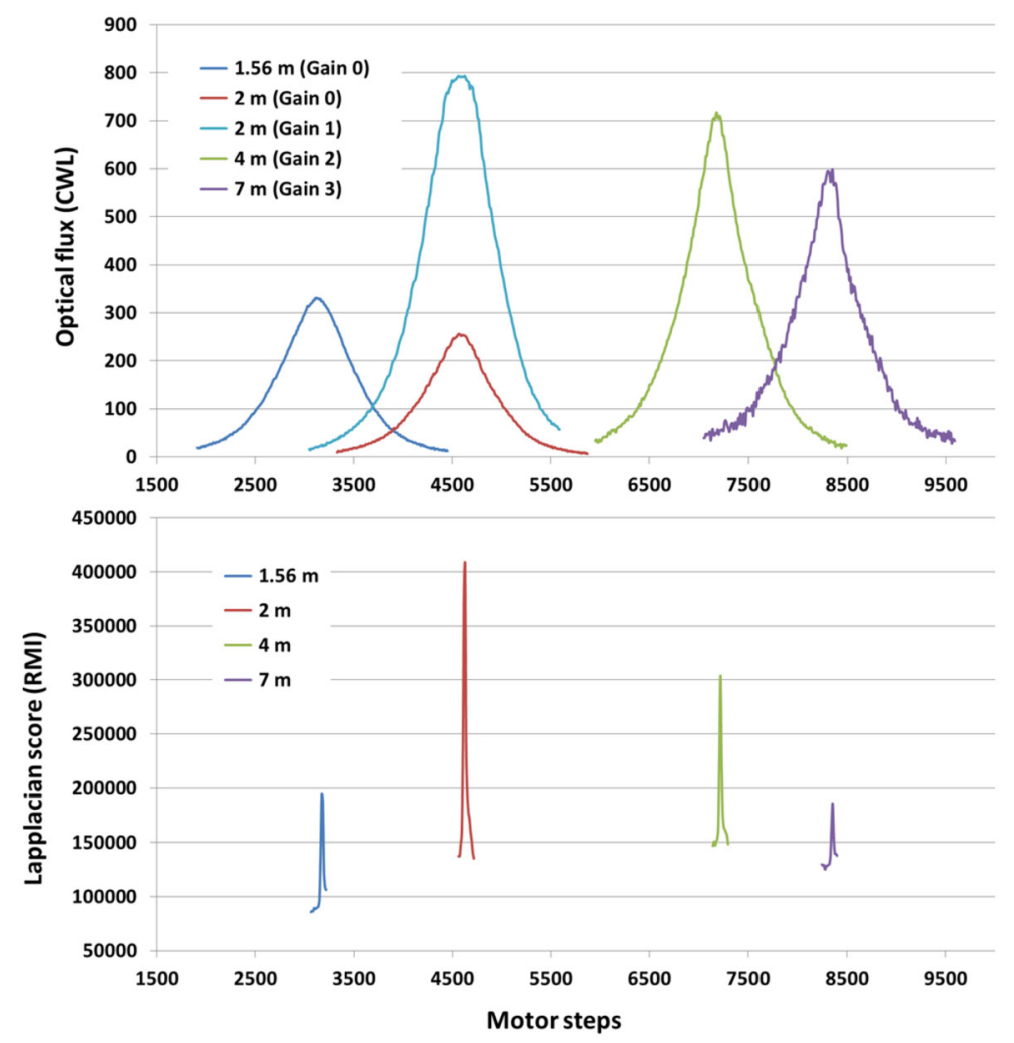

Fig. 74 Autofocus curves for the same target at four distances. (Top) CWL autofocus curves. At $2 \mathrm{~m}$, the measurement is repeated and ended up with a different gain, but the same focus to within 1 step. (Bottom) RMI autofocus curves

at $852 \mathrm{~nm}$ (see Sect. 4.4.5). It is modulated at $10 \mathrm{kHz}$; the light reflected by the target is detected by a photodiode inside the Beamsplitter, and is demodulated using a Finite Impulse Response (FIR) filter at $100 \mathrm{kHz}$. To be independent of the phase shift introduced by the modulation, the signal is convolved by sine and cosine functions on which the FIR is applied. The root mean square of the two signals is proportional to the CWL signal and independent of the phase shift. The FIR is defined by 363 coefficients, and the SNR is set to $80 \mathrm{~dB}$. The onboard processing of the AF curve involves stationary wavelets to filter the data. The algorithm we have implemented for this undecimated cubic spline wavelet transform is described by Starck et al. (2007). The curve is mirrored at both edges to avoid discontinuities. It is best described as an asymmetric Moffat function. Therefore the algorithm determines in the wavelet space the location of the maximum of the CWL curve, which turns out to be the closest position to the maximum of the Moffat function.

In practice, the process begins at the start position of the focus table and moves to the seed given by the Navcams (minus 250 steps to account for the LIBS-to-CWL offset, see below), which has been converted by the Body Unit from millimeters to motor steps. There, the best gain is determined (Fig. 75). The seed is at the center of the scan range. The motor returns to the beginning of the scan zone and starts sampling, typically 255 points, with one point every 10 steps. At the end of the scan, the CWL autofocus curve is analyzed (see below) and the motor returns to the position of best focus. Figure 74 (Top) shows CWL AF 


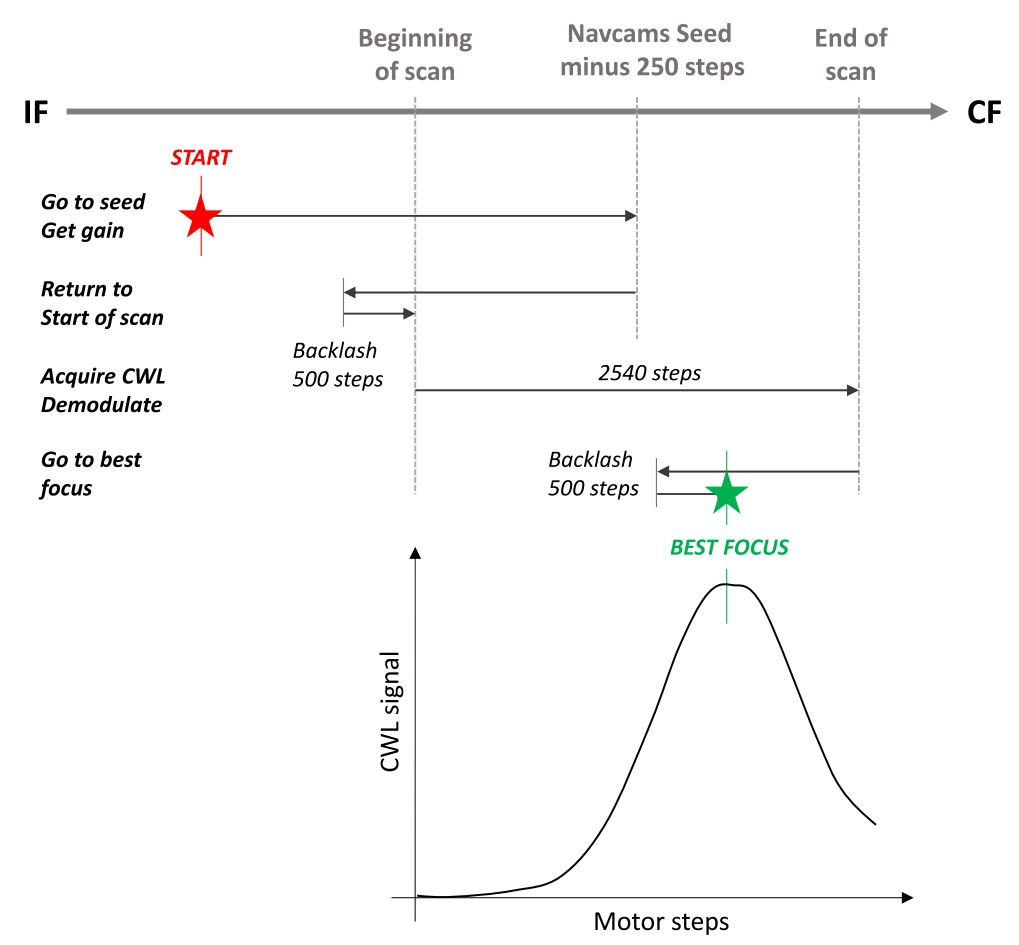

Fig. 75 (Top) Principle of the CWL autofocus mode, when the start of the activity is on the IF side of the scan area (red star), other configurations are possible. (Bottom) The autofocus curve as it should be recorded by the instrument and the calculation of the best focus (green star). It starts from the distance seed given by the Navcams minus a fixed number of steps to account for the LIBS-to-CWL offset

curves on the same target at different distances. At $2 \mathrm{~m}$, the algorithm runs with two different gains. At longer distances, peaks are sharper and noisier.

The two autofocus techniques are very complementary. The CWL autofocus scans a large range of motor steps (typically 2540 steps); therefore it is less affected by the accuracy of the seed. It can run at night or on very uniform targets, but only to $7 \mathrm{~m}$. Compared to the RMI AF however, it is faster but less accurate. The RMI autofocus scans a much smaller range of motor steps (typically 154 steps). So its success depends largely on the accuracy of the seed. It runs only in daylight but at any distance to infinity. It needs targets with moderateto high-contrast structures, such as rocks or soils.

The actual implementation of these techniques is complex, as both had to account for the limit switches and hysteresis (see Sect. 4.3.4). On top of that, the focus stage loses steps each time the motor changes direction. This so-called backlash is compensated by making a large number of steps in the opposite direction each time the system returns to close focus, for instance, to go at the start of the scan or to return to the optimized focus position. Note that the focus scans are made in the direction of the close focus with SuperCam, while it was made in the direction of infinity for ChemCam. This allows the backlash compensation to be applied even at very close distance.

It is important to emphasize that the SuperCam telescope has three non-coincident focal points: for the RMI, the red laser beam, and the CWL (see Sect. 4.4.1). When the red laser beam is focused, by design, the telescope is also focused to collect light into the fiber and 
the IRS. The RMI autofocus procedure yields the best image. A small RMI-to-LIBS offset ( $\sim 25$ steps) has been checked at different distances and temperatures. The position returned by the CWL autofocus is also compared to the best focus for LIBS: a larger CWL-to-LIBS offset ( $\sim 250$ steps) has been tabulated with temperature and distance. These offsets are temperature-dependent and are implemented automatically onboard to move from the different autofocus positions to the best focus positions for LIBS, VISIR, and TRR/L.

\subsection{Design Idiosyncrasies}

There are a few idiosyncrasies inherent to the design of the Mast Unit. We report briefly on three of them that are worth attention when operating the instrument.

\subsubsection{Exclusion Zone}

In LIBS mode, a small fraction of the red laser beam $(\sim 4 \%)$ always reflects off the two faces of the Schmidt plate. The reflected rays focus inside the telescope, most often in air, with no consequence. When the telescope focuses at a distance between $1.56 \mathrm{~m}$ and $1.61 \mathrm{~m}$, called the "exclusion zone", these ghost reflections get focused at the center of the secondary mirror. In this case, the irradiance is high enough to ablate the secondary mirror coating and its reflective layer. Even though the center of the mirror is conjugated to the telescope's central obstruction (i.e., not part of the telescope's optical path), care must be taken to limit the number of LIBS laser shots in this configuration to avoid the ejection of material onto the useful area of the secondary mirror. As a result of damage studies on similar coatings, and using feedback from ChemCam, the following Flight Rule was created: "Do not fire the LIBS laser more than 100,000 times in the exclusion zone with pumping current $\leq 110 \mathrm{~A}$. Do not fire the LIBS laser more than 2,000 times in the exclusion zone with pumping current $>110$ A". This "laser exclusion zone" issue is known from ChemCam, which has two separate exclusion zones, including one on the surface of Mars, at around $\sim 2 \mathrm{~m}$. SuperCam was designed to eliminate the latter one. Regrettably, SuperCam's calibration targets are at a distance from 1.54 to $1.57 \mathrm{~m}$, tangent to the exclusion zone. Thus, the number of LIBS laser shots on the calibration targets will be recorded as a consumable during operations.

\subsubsection{Sun-Safety}

One objective of the Mast-Unit telescope is to concentrate focused light collected by the $110 \mathrm{~mm}$ diameter aperture into a $300 \mu \mathrm{m}$ core fiber (LIBS, TRR/L), a $400 \mu \mathrm{m}$ pinhole (IR), and onto the surface of a CMOS image sensor (RMI) simultaneously. Needless to say, if the RSM is pointing at the Sun and SuperCam's telescope is focused at infinity, the telescope optics could be destroyed. And when the telescope is not focused at infinity, sunward pointing still heats some hardware parts. On the other hand, there are reasons for the project to point the RSM at or near the Sun. These include rover attitude control and opacity measurements, performed by the other cameras on the RSM. In addition, an RSM fault that leaves the mast immobile, waiting for ground control, could accidentally allow the Sun to pass through the telescope's field of view. Therefore SuperCam needed a "sun-safety strategy".

Numerical modeling of the optical design provided details on its vulnerability to the Sun. Depending on the focus position, Sun angles between $0^{\circ}$ and $0.7^{\circ}$ relative the optical axis concentrates intense light on several optical surfaces, from the entrance of the telescope to the fiber. From $2^{\circ}$ to $8^{\circ}$, sunlight concentrates on the secondary mirror baffle, independently 
Fig. 76 Simulations of irradiance on different optics (Top) and absorbed flux (Bottom) as a function of the telescope focus distance, for a $600 \mathrm{~W}$ sunlight source at noon. In red, across the two graphs, the Sun safe position

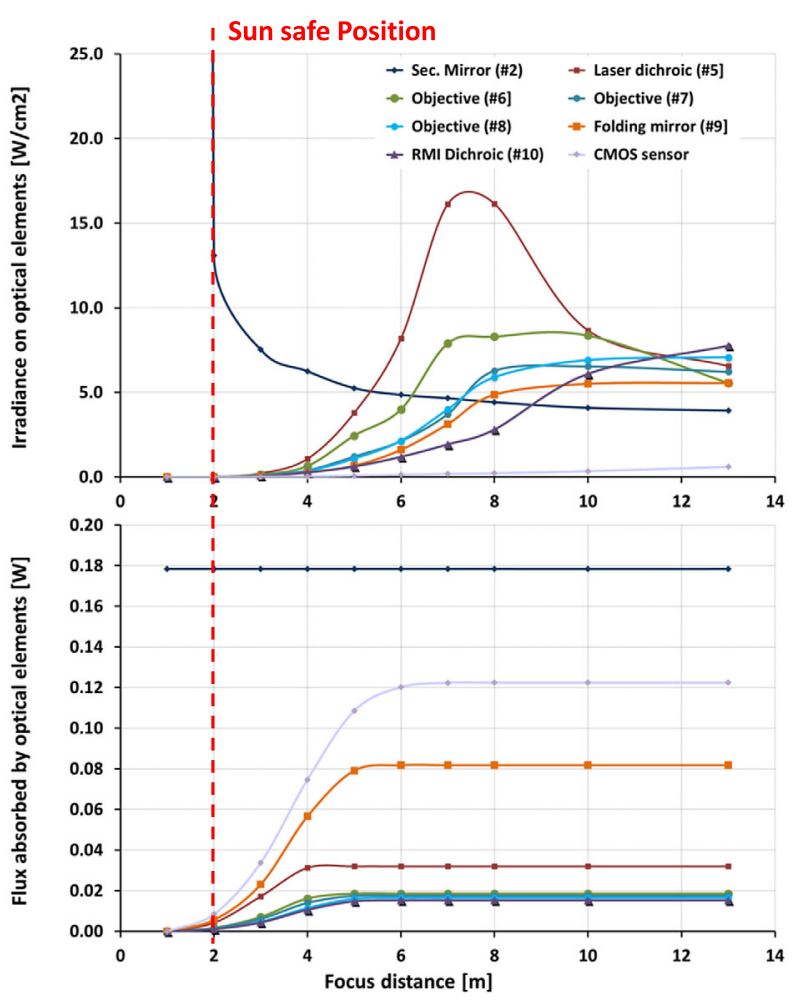

of the focus. The latter case is less critical, as the baffle is made of aluminum. Figure 76 (top) highlights the irradiance on each optical surface for a $600 \mathrm{~W} / \mathrm{m}^{2}$ input (Mars summer, noon) along the optical axis as a function of the telescope focus distance. Around $7 \mathrm{~m}$, a hot spot forms on the laser dichroic. At $2 \mathrm{~m}$, the hot spot is on the secondary mirror baffle, a piece of aluminum. The bottom graph is more important, as it simulates the flux absorbed by the different optics. Because it is not reflective, the RMI CMOS absorbs a lot, and the baffle too. In the end, we decided on $2 \pm 0.2 \mathrm{~m}$ to be the sweet spot, called the "Sun-safe position". With this decision, we combined the two risks mentioned above in one: whatever the configuration, the baffle takes the heat.

Qualification studies, mostly inherited from ChemCam, show that the secondary mirror must stay below $90{ }^{\circ} \mathrm{C}$ (with margins). To be able to withstand all scenarios with no constraint on the operations (a project request), as long as the telescope is in its Sun-safe position, we have worked to reduce the heat absorbed by the baffle $(-30 \%)$ and to increase the thermal conductance of the mirror support by a factor $\sim 2$ compared to ChemCam. We have checked that there is no increase of stray light on the RMI. We have also implemented a PRT for the RCE to monitor the Secondary Mirror temperature (but there is no active control).

In the worst-case situation (when the instrument is tracking the Sun), simulations predict $67{ }^{\circ} \mathrm{C}$ on the secondary mirror, and $84^{\circ} \mathrm{C}$ on the baffle. Acceptance thermal tests measure $66{ }^{\circ} \mathrm{C}$ on the secondary mirror and $83{ }^{\circ} \mathrm{C}$ on the baffle (see Table 6 and Sect. 4.6.2). The agreement is sufficiently good to conclude that SuperCam can sustain a full day of nonoperational sun tracking. Models also predicted that SuperCam will be able to operate for one hour and then track the Sun for 5 hours with significant thermal margins. 
The Mars 2020 Mission System has flight rules and procedures to make sure that the RSM is never pointing less than $0.7^{\circ}$ off the Sun, with margins, when the Mast Unit is not in a Sun safe configuration. The margins are still under discussion with the project. To implement this rule, the Body Unit keeps track of the position of the secondary mirror on its stage at all times. A flag is set when SuperCam is Sun safe and it is reported to the rover in every SuperCam command reply. When the instrument is turned off nominally, the Mast Unit always returns the secondary mirror to the Sun-safe position.

\subsubsection{Stabilization of the $20 \mathrm{~V}$}

During IRS acquisitions, the $20 \mathrm{~V}$ line that generates the RF signal must be stable to $\pm 1 \mathrm{~V}$. This voltage is created by the third converter of the LVPS board (see Fig. 65) together with the $30 \mathrm{~V}$. We find that the $20 \mathrm{~V}$ is stable only if the two lines are well balanced. Therefore, we need to load the $30 \mathrm{~V}$ line during IRS acquisitions. Two solutions to this idiosyncrasy have been implemented. The first solution is to supply the focus motor with $0.6 \mathrm{~A}$ on both phases. The motor does not move; the energy is dissipated in the motor windings. The second solution is to run current through a $162 \mathrm{Ohm}$ resistor mounted on the EBOX structure, thanks to a dedicated switch on the DPU board. Both solutions are functional; the first is the preferred one. Under worst-case conditions, we have checked that the motor does not overheat above its $67^{\circ} \mathrm{C}$ qualification temperature.

\subsection{Assembly, Integration, and Tests}

Here are a few details on how the instrument was integrated and tested, while complying with Planetary Protection and Contamination Control regulations. Contamination control ensures the stability of optical properties with time, during the integration process, prelaunch, launch, cruise, and landing, and operations on the surface of Mars. The Mast Unit was entirely developed in France. Several early models (EDU, EQM) went to LANL for various coupling tests. The FM went directly to JPL, where it met the Body-Unit flight model on the rover for a six month Assembly, Tests, and Launch Operations (ATLO) campaign.

\subsubsection{Integration Flow}

The integrated sub-systems (Laser, Imager cube, IRS, Translation stage, Shutter, and Microphone) were delivered to IRAP fully tested and ready to be installed in the Mast Unit. Similarly the electronic boards (LVPS, DPU, IR, Laser, and OBOX FEEs) went through functional tests, and performance verifications at three temperatures. Some elements had to be matched (or tuned) and this required the adjustment of some electronic resistors and capacitors. These included the IR board with the IR box, and the laser board with the flight laser. Figure 77 illustrates the whole integration flow.

Once tested, the boards were initially mounted onto a breakout structure. Initial tests were done mating the LVPS and DPU boards, and the IR board with the IR box. The functionality of the overall control by the DPU board was tested. In parallel, the laser board was optimized (timing, high-voltage) to extract the maximum energy at $532 \mathrm{~nm}$ and $1064 \mathrm{~nm}$ at cold temperatures. Once validated together, the boards were conformal coated (MAPSIL or Parylene) and the harnesses were custom shaped. All boards were then integrated into the EBOX structure.

The assembly of the OBOX required a significant amount of work. The subsystems (Green Galilean expander, Red Galilean expander, Objective) were assembled on their own, 

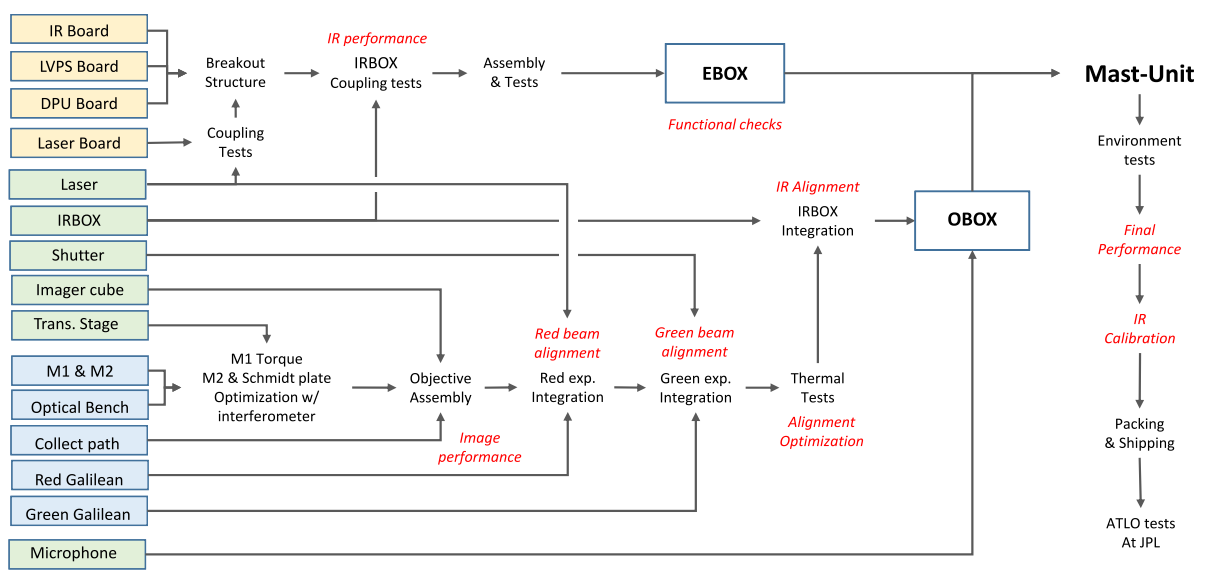

Fig. 77 Simplified schematic of the EBOX and OBOX assemblies. Yellow: Electronic boards, Green: Integrated subsystems, Blue: Opto-mechanical parts. Red text is for critical tests

and their optical performance was checked. The telescope components (primary and secondary mirrors, Schmidt plate) were assembled first with the translation stage. We used micron-size shims to make sure that it stayed aligned with distance, since the telescope boresight is the main optical axis of the whole instrument. The primary mirror was torqued with extreme care while being monitored by an interferometer to minimize wavefront errors due to mechanical deformation. The alignment of the secondary mirror and the centering of the Schmidt plate that closes the telescope were also optimized in front of an interferometer. Once the imager cube and the objective were mounted, the imaging performance was checked at several distances. This step reveals the optical quality of the telescope. The laser and red expander were integrated onto the optical bench. It is critical to avoid any vignetting of the laser and to align its axis on the telescope boresight. At that point, the ability of the telescope to focus the red beam was set, and the collection was aligned with the LIBS. The green expander was integrated with the same care. Its alignment included an offset at room temperature (see Sect. 4.5.2, 2nd paragraph), characterized by thermo-elastic tests. The CW laser was also aligned along the same axis. Once integrated, we checked all of the performance metrics at different temperatures. The IR spectrometer was finally integrated onto the optical bench, and the microphone came last. During this six-month process, systems of the OBOX were driven by Ground Support Equipment (GSE) of various types. Electrical GSE controlled the OBOX when it was not possible to do so with the EBOX breakout structure. Mechanical GSE protected mirrors and lenses, and reduced risk during assembly. Optical GSE aided in the optical characterization of the OBOX. The ground software on the electrical GSE was the same for all configurations. Reference scripts were defined for more efficiency and replicability.

Once the EBOX and OBOX were integrated together, a set of final reference tests at room temperature was acquired. After environmental testing (Vibration, shocks, thermal vacuum, reference test) the same tests were performed again.

\subsubsection{Planetary Protection (PP)}

The Mast Unit is located within the RWEB, which is equipped with a HEPA filter. For that type of enclosure, the bioburden requirement for the Mast-Unit surfaces is $1,000 \mathrm{spores} / \mathrm{m}^{2}$ 
maximum. The specification for the microphone is much more stringent, at $300 \mathrm{spores} / \mathrm{m}^{2}$ maximum, since it is the only part of the Mast-Unit outside the RWEB and in direct contact with the Martian environment. The general approach for PP is a microbial reduction (isopropyl alcohol cleaning), bioassay (sampling), and contamination prevention (bagging, etc.). We followed JPL best practices for ISO 5 or ISO 7 class cleanrooms in which the Mast Unit was integrated. Sampling for bioassay was performed by an independent sub-contractor following ECSS-Q-ST-70-55C. JPL also checked the PP compliance regularly by Adenosine Triphosphate (ATP) swabbing.

\subsubsection{Contamination Control (CC)}

Contamination was controlled to avoid self-contamination within the instrument, or contamination of other instruments on Perseverance. The Mast Unit is sensitive to particulate and molecular contamination. On top of the risk of losing optical transmission (in particular in the UV and IR), some of the Mast-Unit optics see high laser fluences and could suffer from laser-induced contamination. Moreover, molecular contamination needed to be controlled to avoid any parasitic Raman signal.

Cleanliness requirements were particulate Visibly Clean-Highly Sensitive (VC-HS) and molecular contamination $\leq 100 \mathrm{ng} . \mathrm{cm}^{-2}$. JPL also specified an outgassing requirement ( $\leq 30 \mathrm{ng} \cdot \mathrm{cm}^{-2} \cdot \mathrm{h}^{-1}$ with the MU at $50{ }^{\circ} \mathrm{C}$ and a Thermoelectric Quartz Crystal Microbalances (TQCM) at $-50{ }^{\circ} \mathrm{C}$ ).

The Mast-Unit control plan was based on good practices that ensured the instrument met the requirements:

- In the design phase: careful choice of materials and processes. This information is detailed in the Material Identification and Usage List (MIUL).

- In the integration phase: systematic bakeout of the hardware (e.g., OBOX structure after bonding of heaters) and the subsystems, to reduce outgassing during the first thermal tests under vacuum. The integration and most of the tests were performed in an ISO 7 class cleanroom with appropriate garments (see Fig. 15).

- In the test phases: cleaning and bakeout of the GSE (harnesses, etc.) and facilities.

- Particulate and molecular witnesses were exposed and measured during all phases of integration, testing, transportation and storage.

The Mast Unit FM passed all PP and CC inspections successfully at JPL upon delivery.

\section{Test Validation and Conclusions}

SuperCam embeds five different kinds of investigations (LIBS, TRR/L, VISIR, RMI, and MIC) that are part of the classical range of analytical techniques in a geoscience laboratory, except perhaps the microphone. Efforts are made to miniaturize each of them without compromising their significance for Mars exploration. However, accommodation constraints and the tradeoffs inherent to the search for stability and reliability made it necessary to frame their range of performance, in terms of distance range, sensibility, accuracy, or precision. Here the final characteristics of the Mast Unit, before it leaves Earth, are accounted for, starting with technical resources.

At the end of the development, all Mast-Unit measurement and functional requirements, also called L5 requirements, were meticulously validated, first during coupling tests with the 
Table 8 Mast-Unit key resources, and when needed, the SuperCam ones

\begin{tabular}{llc}
\hline SuperCam & Mass (sum of sub-systems) & $10.8 \mathrm{~kg}$ \\
Mast-Unit & Mass (measured) & $\mathbf{6 . 1} \mathbf{~ k g}$ \\
Body-unit & Mass (measured) & $4.4 \mathrm{~kg}$ \\
Cal. Targets & Mass (measured) & $0.3 \mathrm{~kg}$ \\
\hline SuperCam & Power (sum of sub-systems) & $70 \mathrm{~W}$ \\
Mast-Unit & Power (max) at 28 V & $\mathbf{2 7 ~ W}$ \\
Body-Unit & Power (max) at 28 V & $43 \mathrm{~W}$ \\
SuperCam & Energy (Typ. case) & $18 \mathrm{Whr}$ \\
\hline SuperCam & Main switch (max) at 28 V & $2.5 \mathrm{~A}$ \\
Mast-Unit & Survival (avg.) & $\mathbf{0 . 8 ~ A}$ \\
Body-Unit & Decon heaters (max) & $0.9 \mathrm{~A}$ \\
\hline SuperCam & Uncompress data vol. (typ. case) & $27 \mathrm{Mbytes}$ \\
Mast-Unit & FPGA gate utilization & $\mathbf{3 0 \%}$ \\
Mast-Unit & Boot PROM utilization & $\mathbf{1 7 \%}$ \\
Mast-Unit & Nonvolatile Memory Utilization & $\mathbf{1 3 \%}$ \\
Mast-Unit & Volatile Memory Utilization (5 images) & $\mathbf{6 3 \%}$ \\
\hline & &
\end{tabular}

Body-Unit EQM at IRAP, Toulouse, and then during the acceptance thermal vacuum campaign at CNES, Toulouse. Performance requirements, also called L4 requirements, were primarily validated at JPL, as part of the ATLO campaign when the Mast Unit was coupled to the Body-Unit flight model. Results are reported by Wiens et al. (this journal). However, some L4 requirements relied solely on the Mast Unit. For more details about the IRS, a comprehensive IR calibration was performed at LESIA, France, before the delivery of the Mast Unit to JPL. It is reported by Royer et al. (2020).

\subsection{Status of Technical Resources}

This section provides a technical resource budget and as-built data for the Mast Unit, and when necessary, for the whole instrument for consistency (Table 8). The mass numbers are the measured values of the as-built flight models: $10.8 \mathrm{~kg}$ for SuperCam, including $6.1 \mathrm{~kg}$ for the Mast Unit.

The maximum power at $28 \mathrm{~V}$ bias is $70 \mathrm{~W}$ for SuperCam, including $27 \mathrm{~W}$ for the Mast Unit. The maximum current corresponds to $2.5 \mathrm{~A}$, well below the $10 \mathrm{~A}$ rating of the rover power switch. There are independent lines for the Mast-Unit survival and Body-Unit decontamination heaters, with their own current rating at $2 \mathrm{~A}$ and $4 \mathrm{~A}$, respectively.

SuperCam has several operating modes, each one having its own power requirement and duration. Consequently, the instrument energy requirement depends on the sequence of modes used. Hence a notional 25 min sequence is proposed: Body Unit on, TEC cooling from $30{ }^{\circ} \mathrm{C}$ RAMP, Mast Unit on (when CCDs approach their operating temperature), lasers warm up, CWL AF, RMI, 30 LIBS spectra at $3 \mathrm{~Hz}$ with microphone, 10 collects of 10 Raman spectra, a VISIR spectrum (128 spectels for IRS), RMI image, transfer of data to RCE, and turn both units off. This sequence does not necessarily represent a sequence of operations that would actually be executed on Mars. Instead, its role is to provide some idea of how SuperCam's resources have evolved from early development to final delivery. Based on the notional sequence, the Mast Unit will use 18 W.hr. 
The uncompressed data volume for the notional sequence would be 27 Mbytes. By far the largest data volume is generated by the RMI images, 8 Mbytes each. As a reminder, $2.5 \mathrm{~min}$ of acoustic data at $25 \mathrm{kHz}$ can also produce 8 Mbytes of data. All RMI and spectrometers data are expected to be highly compressible. Experience with ChemCam has shown that both LIBS data and images can be compressed from 4:1 to as much as 10:1 without loss of science information.

The Mast-Unit FPGA processor resources include 32 kbytes of non-volatile PROM memory to store the BFSW. There is also 512 kbytes of MRAM non-volatile memory to store the AFSW and run time application parameters. Finally, the processor resources include 64 Mbytes of SDRAM volatile memory to store data collected by the Mast Unit and to support all run time applications. All utilization resources are given in Table 8. Flight software CPU utilization is not a useful resource to track for the Mast Unit since this processor simply responds to commands issued by the Body Unit. The Body Unit then waits until the Mast Unit completes the command before issuing the next command. The number of CPU cycles to complete a command versus the number of cycles used waiting for the next command depends on the command being processed.

\subsection{Checking Functional and Performance Requirements}

A comprehensive set of Verification Activities (VAs) was defined to validate the functionalities and high-level technical requirements of the Mast Unit. All VAs were first run at room temperature before mechanical and thermal tests. A subset was run after these tests to validate the robustness of the instrument design. Most of them were performed on the different dwells during the two-week long thermal vacuum tests (TVAC). The Mast Unit was operating under 7 mbar of $\mathrm{N}_{2}$ over the AFT range. Validation activities cover:

- Telescope focus:

o Positions of close and infinite focus, end switches hysteresis, distance vs. steps laws;

o CWL AF and RMI AF, validation of algorithm, duration, repeatability;

o Offset laws between CWL AF, RMI AF, and best position for LIBS;

o CW laser power.

- Red and green laser beams:

o Energy vs. pumping current, long and short bursts;

o Red laser beam irradiance and green laser beam footprint;

o Long and short bursts, margins to relaxation mode;

o Alignment of red and green laser beams, CWL, all with fiber, RMI, and IRS;

o Timing of laser beams relative to the Body-Unit spectrometers.

- Light collect:

o Relative optical transmission to fiber, RMI, and IRS;

o Fiber FOV and DoF;

o IR FOV and DoF.

- Remote Micro-Imaging:

o Dark current, offset level;

o FOV, DOF, and flat field characterization;

o Spatial resolution (absolute around the center, and relative on the edges);

o Auto-exposure and HDR algorithms.

- Microphone:

o Gains, background noise;

o Electromagnetic noise with LIBS;

o Synchronization with LIBS. 
Table 9 Overview of key characteristics for each investigation. A check mark is for the parameters validated at the Mast-Unit level. Others require the Body Unit, labeled "BU" (see Wiens et al., this journal). All functions need command \& data handling from the Body Unit

\section{LIBS}

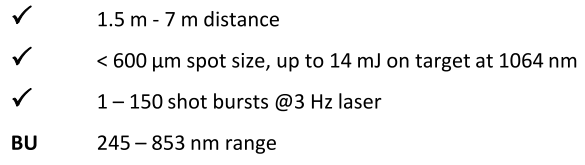

BU $\quad 0.15-0.65 \mathrm{~nm}$ resolution, SNR $>60 @ 767 \mathrm{~nm}$ (w/ 30 shots)

\section{TRR and TRL}

$1.5 m-7 m$ distance

$\sim 0.7 \mathrm{mrad}$ FOV, $>9 \mathrm{~mJ}$ on target at $532 \mathrm{~nm}$

1-200 shot bursts@10 Hz laser

$150-4400 \mathrm{~cm}^{-1}$ range

$<12 \mathrm{~cm}-1$ resolution, $\mathrm{SNR}>20$ ( $\mathrm{w} / 200$ shots)

Time sweep: $100 \mathrm{nsec}$ windows, delays up to $10 \mathrm{msec}$

\section{VIS}

$1 \mathrm{~m}$ to $\infty$ range

$\sim 0.7 \mathrm{mrad}$ FOV

$400-853 \mathrm{~nm}$ range

BU

$0.15-0.65 \mathrm{~nm}$ resolution, SNR $>50 @ 800 \mathrm{~nm}$

IR

$1 \mathrm{~m}$ to $\infty$ range

$\sim 1.2 \mathrm{mrad}$ FOV, $1.3-2.6 \mu \mathrm{m}$ range

$<32 \mathrm{~cm}^{-1}$ res., SNR $>56$, up to 256 spectels max. sampling

RMI

$1 \mathrm{~m}$ to $\infty$ range

Focal length $563 \mathrm{~mm}, \mathrm{f} / 5$

19 mrad FOV, iFOV $10 \mu \mathrm{rad}$, standard RGB color filtering

Spatial resolution $<80 \mu \mathrm{rad}$

Contrast $>20 \%$ at 20 line pairs $/ \mathrm{mm}$ over half-FOV

MIC

$1 \mathrm{~m}$ to $4 \mathrm{~m}$ distance

$100 \mathrm{~Hz}-10 \mathrm{kHz}$ range, sampling at $25 \mathrm{kHz}$ or $100 \mathrm{kHz}$

Standalone mode ( $2.7 \mathrm{~min}$ rec. $\max$ )

LIBS shot recording (up to 150 shots)

- Infrared Spectrometer:

o TEC cool down;

o Dark current, RF profile.

The TVAC results validate (and calibrate) all Mast-Unit L5 requirements that are defined in Sect. 3.3, for different temperatures between $-40{ }^{\circ} \mathrm{C}$ and $+35^{\circ} \mathrm{C}$, and distances between $2 \mathrm{~m}$ and $7 \mathrm{~m}$. They help to redefine some use cases, for the margins to the laser relaxed mode for instance. Results are presented all through with this publication.

\subsection{Conclusion}

The Mast Unit had many challenging aspects to its design: one pulsed laser with two beams, one CW laser, two mechanisms, more than 30 optical parts, micrometer alignments, a wide 
wavelength range, two fast-switching high-voltages, high-current lines, radio-frequency signals, low-noise readout requirements, thermal systems for both heating and cooling, numerous commercial off-the-shelf components needing special flight qualification, and a number of new or unusual technologies (CMOS, AOTF, IR photodiodes, Pockels cells). They were all individual challenges to develop and qualify. Considering the tight schedule, constrained resources (mass, volume, and power), the thermal environment, and the efforts to make all systems to work together, the development of SuperCam Mast Unit has been challenging.

As a whole, the Mast Unit meets all its requirements from $2 \mathrm{~m}$ to $7 \mathrm{~m}$, and at the calibration target $(1.56 \mathrm{~m})$ location. Table 9 summarizes SuperCam's key characteristics, and the ones that have been exclusively validated at the Mast-Unit level. The autofocus capability is not explicitly shown, but its role is paramount to the success of all investigations, except for the microphone. For this reason, it has been duplicated as the RMI and CWL AF methods, which still require the same focus stage.

For LIBS, the challenge was to generate enough irradiance at $1064 \mathrm{~nm}$ on the target in the operational temperature range. For TRR/L, beyond the generation of a collimated beam at $532 \mathrm{~nm}$, the main hurdle was to maintain the beam alignment at all distances and temperatures. For both techniques, the returned photons (LIBS spark, Raman and luminescence signals) are collected and sent to the Body Unit, where the spectral analysis is performed. The Body Unit spectrometers define the SNR and resolution, which are critical for the success of the scientific results. On both parts of the instrument, the development of the VIS reflectance presents fewer challenges when the LIBS functions are realized.

The IR range is new and includes several challenges, not so much the collection of light, but its spectral dispersion. AOTFs are not new in space but they are delicate to drive, in particular when the two diffracted orders are recorded and need to be angularly stabilized; additionally, the commercial IR photodiodes were difficult to qualify, which is one reason for designing the instrument with redundant sensors. The electromagnetic environment of these photodiodes has been treated with the utmost care to obtain an electronic noise $<0.1 \mathrm{pA}$.

The RMI is more than a duplication of ChemCam's imager with color filters. The type of detector was changed to a CMOS, which has its own challenges with well depth, requiring HDR. It is always difficult to design a telescope whose main purpose is to focus light at very small scales, and to obtain a relatively large field of view for imaging at the same time. A new primary mirror relative to ChemCam helped to achieve the required resolution at all temperatures.

Finally, the microphone was added late in the development of the Mast Unit. Except for a particular thermal environment (going down to $-125^{\circ} \mathrm{C}$ ), the key difficulty was the electromagnetic compatibility with the laser and the accommodation on the RWEB.

This paper is the first in a series of two publications on the design of the SuperCam instrument, plus one on the onboard calibration targets (Manrique et al., this journal). The reader should refer to Wiens et al. (this journal) for a more thorough description of the Body Unit and a discussion of the final performance of the instrument.

Acknowledgements Many other people helped with this project in addition to the co-authors, and we are most grateful for their support. This project was supported in the US by the NASA Mars Exploration Program, and in France by CNES, CNRS, and local Universities.

The whole team is very grateful to our Mast-Unit chief optical engineer and friend, Laurent Parès, who designed SuperCam. He passed away in February 2020.

Authors' contributions All authors contributed to either the proposal or the development and testing of the SuperCam instrument as described in this paper. 
Funding In France was provided by the Centre National d'Etudes Spatiales (CNES). Human resources were provided in part by the Centre National de la Recherche Scientifique (CNRS) and universities. Funding was provided in the US by NASA's Mars Exploration Program. Some funding of data analyses at Los Alamos National Laboratory (LANL) was provided by laboratory-directed research and development funds.

Data availability Data presented in the Results section of this paper are being made available to the Planetary Data System Geosciences Node under Mars 2020/SuperCam.

\section{Code availability Not applicable.}

Conflicts of interest/Competing interests The authors declare that there are no conflicts of interest or competing interests.

Publisher's Note Springer Nature remains neutral with regard to jurisdictional claims in published maps and institutional affiliations.

Open Access This article is licensed under a Creative Commons Attribution 4.0 International License, which permits use, sharing, adaptation, distribution and reproduction in any medium or format, as long as you give appropriate credit to the original author(s) and the source, provide a link to the Creative Commons licence, and indicate if changes were made. The images or other third party material in this article are included in the article's Creative Commons licence, unless indicated otherwise in a credit line to the material. If material is not included in the article's Creative Commons licence and your intended use is not permitted by statutory regulation or exceeds the permitted use, you will need to obtain permission directly from the copyright holder. To view a copy of this licence, visit http://creativecommons.org/licenses/by/4.0/.

\section{Appendix of acronyms}

List of acronyms that are used multiple times away from their definition.
AFSW Application Software
AFT Allowable Flight Temperature
AOTF Acousto-Optic Tunable Filter
ATLO Assembly, Tests, and Launch Operations
AZ Azimuth
BFSW Boot Flight Software
C\&DH Command and Data Handling
CC Contamination Control
CF Close Focus
CMOS Complementary Metal Oxide Semiconductor
CWL Continuous Wavelength Laser
DOF Depth-of-field
DPU Digital Processor Unit
EBOX Electronic Box
EDU Engineering Development Unit
EL Elevation
EQM Engineering Qualification Model
FEE Front End Electronics
FM Flight Model
FOV Field of view
FPGA Field Programmable Gate Arrays
FWHM Full Width at Half Maximum
GSE Ground Support Equipments 


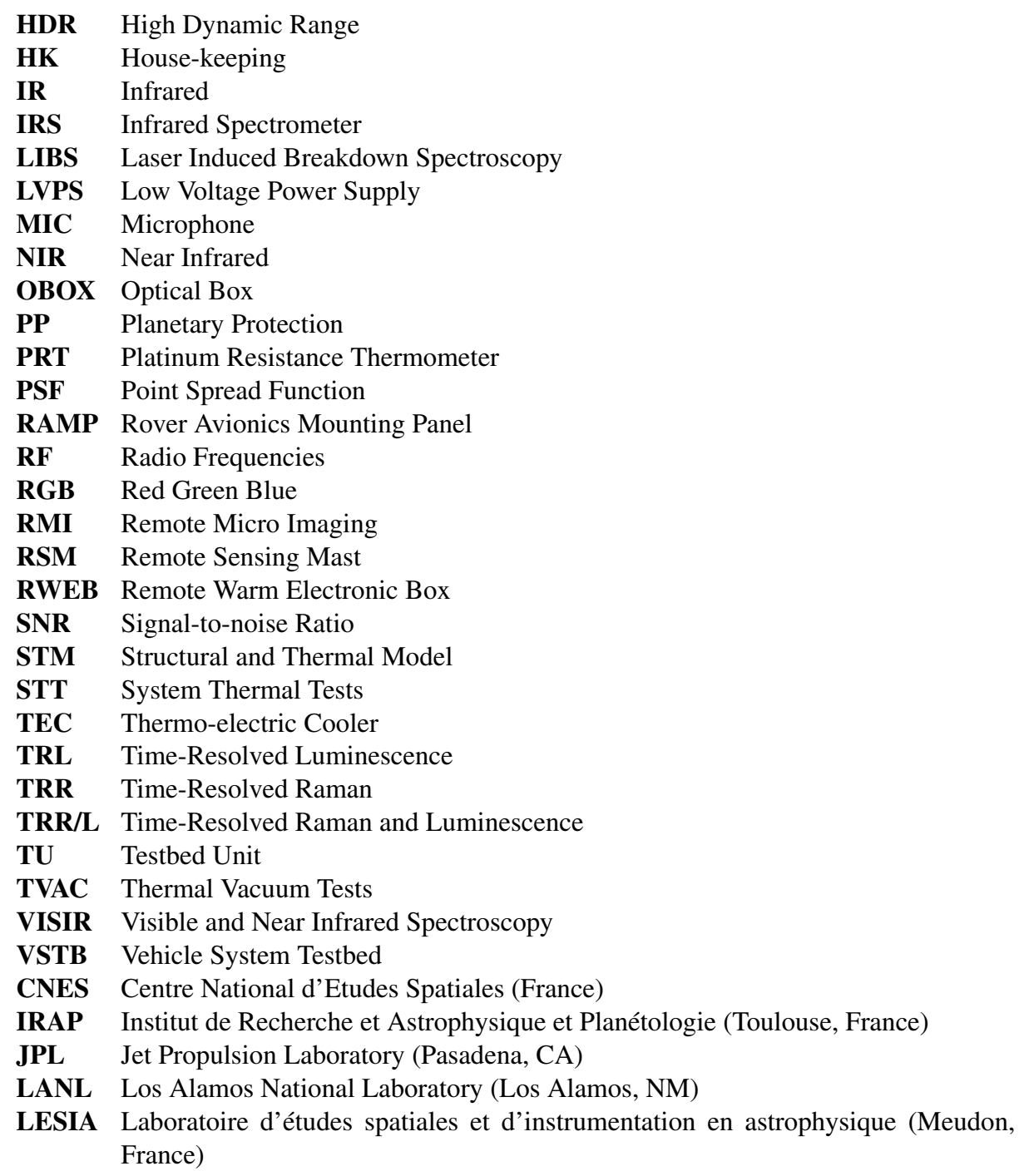

\section{References}

F. Anabitarte, A. Cobo, J.M. Lopez-Higuera, Int. Sch. Res. Not. (2012). https://doi.org/10.5402/2012/285240

D.E. Anderson, B.L. Ehlmann, O. Forni, S.M. Clegg, A. Cousin, N.H. Thomas, J. Lasue, D.M. Delapp, R.E. McInroy, O. Gasnault, M.D. Dyar, S. Schröder, S. Maurice, R.C. Wiens, J. Geophys. Res., Planets (2017). https://doi.org/10.1002/2016JE005164

H.E. Bass, J.P. Chambers, J. Acoust. Soc. Am. (2001). https://doi.org/10.1121/1.1365424

C.C. Bedford, J.C. Bridges, S.P. Schwenzer, R.C. Wiens, E.B. Rampe, J. Frydenvang, P.J. Gasda, Geochim. Cosmochim. Acta (2019). https://doi.org/10.1016/j.gca.2018.11.031

O. Beyssac, Elements (2020). https://doi.org/10.2138/gselements.16.2.117

J.-P. Bibring, Y. Langevin, in The Martian Surface - Composition, Mineralogy, and Physical Properties, ed. by J. Bell III (Cambridge University Press, Cambridge, 2008), p. 153. ISBN-10: 0521866987

J.L. Bishop, in Remote Compositional Analysis: Techniques for Understanding Spectroscopy, Mineralogy, and Geochemistry of Planetary Surfaces (2019). https://doi.org/10.1017/9781316888872.006 
R. Brennetot, J.L. Lacour, E. Vors, A. Rivoallan, D. Vailhen, S. Maurice, Appl. Spectrosc. (2003). https:// doi.org/10.1366/000370203322102816

N.T. Bridges, F.J. Calef, B. Hallet, K.E. Herkenhoff, N.L. Lanza, S. Le Mouélic, C.E. Newman, D.L. Blaney, M.A. Pablo, G.A. Kocurek, Y. Langevin, K.W. Lewis, N. Mangold, S. Maurice, P.-Y. Meslin, P. Pinet, N.O. Renno, M.S. Rice, M.E. Richardson, V. Sautter, R.S. Sletten, R.C. Wiens, R.A. Yingst, J. Geophys. Res., Planets (2014). https://doi.org/10.1002/2013JE004579

B. Chide, S. Maurice, N. Murdoch, J. Lasue, B. Bousquet, X. Jacob, A. Cousin, O. Forni, O. Gasnault, P.-Y. Meslin, J.-F. Fronton, M. Bassas-Portús, A. Cadu, A. Sournac, D. Mimoun, R.C. Wiens, Spectrochim. Acta, Part B, At. Spectrosc. (2019). https://doi.org/10.1016/j.sab.2019.01.008

B. Chide, N. Murdoch, Y. Bury, S. Maurice, X. Jacob, J.P. Merrison, J.J. Iversen, P.-Y. Meslin, M. BassasPortus, A. Cadu, A. Sournac, B. Dubois, R.D. Lorenz, D. Mimoun, R.C. Wiens, Icarus (2020a). https:// doi.org/10.1016/j.icarus.2020.114060

B. Chide, S. Maurice, A. Cousin, B. Bousquet, D. Mimoun, O. Beyssac, P.-Y. Meslin, R.C. Wiens, Spectrochim. Acta, Part B, At. Spectrosc. (2020b). https://doi.org/10.1016/j.sab.2020.106000

M.L. Chithambo, An Introduction to Time-Resolved Optically Stimulated Luminescence (2018). https://doi. org/10.1088/2053-2571/aae5da

S.M. Clegg, R.C. Wiens, R. Anderson, O. Forni, J. Frydenvang, J. Lasue, A. Cousin, V. Payré, T. Boucher, M. Darby Dyar, S.M. McLennan, R.V. Morris, T.G. Graff, S.A. Mertzman, B.L. Ehlmann, I. Belgacem, H. Newsom, C. Ben Clark, N. Melikechi, A. Mezzacappa, R.E. McInroy, R. Martinez, P. Gasda, O. Gasnault, S. Maurice, Spectrochim. Acta, Part B, At. Spectrosc. (2017). https://doi.org/10.1016/j.sab. 2016.12.003

A. Cousin, E. Dehouck, P.-Y. Meslin, O. Forni, A.J. Williams, N. Stein, O. Gasnault, N. Bridges, B. Ehlmann, S. Schröder, V. Payré, W. Rapin, P. Pinet, V. Sautter, N. Lanza, J. Lasue, S. Maurice, R.C. Wiens, J. Geophys. Res., Planets (2017). https://doi.org/10.1002/2017JE005261

D.A. Cremers, L.J. Radziemski, Handbook of Laser-Induced Breakdown Spectroscopy (Wiley, New York, 2006). https://doi.org/10.1002/0470093013

D.P. Cruikshank, L.V. Moroz, R.N. Clark, in Remote Compositional Analysis: Techniques for Understanding Spectroscopy, Mineralogy, and Geochemistry of Planetary Surfaces (2019). https://doi.org/10.1017/ 9781316888872.007

G. David, A. Cousin, O. Forni, P.-Y. Meslin, E. Dehouck, N. Mangold, J. L'Haridon, W. Rapin, O. Gasnault, J.R. Johnson, A.M. Ollila, A.R. Newell, M. Salvatore, T.S.J. Gabriel, R.C. Wiens, S. Maurice, J. Geophys. Res., Planets (2020). https://doi.org/10.1029/2019JE006314

G.T. Delory, J. Luhmann, L. Friedman, B. Betts, Development of the first audio microphone for use on the surface of Mars. J. Acoust. Soc. Am. 121(5), 3116 (2007). https://doi.org/10.1121/1.4782072

J. Dubessy, M.-C. Caumon, F. Rull, S. Sharma, in Raman Spectroscopy Applied to Earth Sciences and Cultural Heritage, ed. by J. Dubessy, M.-C. Caumon, F. Rull (European Mineralogical Union and Mineralogical Society of Great Britain \& Ireland, London, 2012). https://doi.org/10.1180/EMU-notes.12.3

P.H. Edwards, J.C. Bridges, R. Wiens, R. Anderson, D. Dyar, M. Fisk, L. Thompson, P. Gasda, J. Filiberto, S.P. Schwenzer, D. Blaney, I. Hutchinson, Meteorit. Planet. Sci. (2017). https://doi.org/10.1111/maps. 12953

B.L. Ehlmann, C.S. Edwards, Annu. Rev. Earth Planet. Sci. (2014). https://doi.org/10.1146/annurev-earth060313-055024

A. Fau, O. Beyssac, M. Gauthier, P.Y. Meslin, A. Cousin, K. Benzerara, S. Bernard, J.C. Boulliard, O. Gasnault, O. Forni, R.C. Wiens, M. Morand, P. Rosier, Y. Garino, S. Pont, S. Maurice, Spectrochim. Acta, Part B, At. Spectrosc. (2019). https://doi.org/10.1016/j.sab.2019.105687

O. Forni, M. Gaft, M.J. Toplis, S.M. Clegg, S. Maurice, R.C. Wiens, N. Mangold, O. Gasnault, V. Sautter, S. Le Mouélic, P.-Y. Meslin, M. Nachon, R.E. McInroy, A.M. Ollila, A. Cousin, J.C. Bridges, N.L. Lanza, M.D. Dyar, Geophys. Res. Lett. (2015). https://doi.org/10.1002/2014GL062742

R. Francis, T. Estlin, G. Doran, S. Johnstone, D. Gaines, V. Verma, M. Burl, J. Frydenvang, S. Montaño, R.C. Wiens, S. Schaffer, O. Gasnault, L. DeFlores, D. Blaney, B. Bornstein, Sci. Robot. (2017). https:// doi.org/10.1126/scirobotics.aan4582

M. Gaft, G. Panczer, Mineral. Petrol. (2013). https://doi.org/10.1007/s00710-013-0293-3

M. Gaft, R. Reisfeld, G. Panczer, Modern Luminescence Spectroscopy of Minerals and Material. Springer Mineralogy (2015). https://doi.org/10.1007/978-3-319-24765-6. 606 pp.

M. Gaft, Y. Raichlin, F. Pelascini, G. Panzer, V. Motto Ros, Spectrochim. Acta, Part B, At. Spectrosc. (2019). https://doi.org/10.1016/j.sab.2018.11.003

M. Gaft, L. Nagli, I. Gornushkin, Y. Raichlin, Spectrochim. Acta (2020). https://doi.org/10.1016/j.sab.2020. 105989

P.J. Gasda, E.B. Haldeman, R.C. Wiens, W. Rapin, T.F. Bristow, J.C. Bridges, S.P. Schwenzer, B. Clark, K. Herkenhoff, J. Frydenvang, N.L. Lanza, S. Maurice, S. Clegg, D.M. Delapp, V.L. Sanford, M.R. Bodine, R. McInroy, Geophys. Res. Lett. (2017). https://doi.org/10.1002/2017GL074480 
J.R. Johnson, J.F. Bell, S. Bender, D. Blaney, E. Cloutis, L. DeFlores, B. Ehlmann, O. Gasnault, B. Gondet, K. Kinch, M. Lemmon, S. Le Mouélic, S. Maurice, M. Rice, R.C. Wiens, Icarus (2015). https://doi.org/ 10.1016/j.icarus.2014.02.028

J.R. Johnson, C. Achilles, J.F. Bell, S. Bender, E. Cloutis, B. Ehlmann, A. Fraeman, O. Gasnault, V.E. Hamilton, S. Le Mouélic, S. Maurice, P. Pinet, L. Thompson, D. Wellington, R.C. Wiens, J. Geophys. Res., Planets (2017). https://doi.org/10.1002/2016JE005187

A. Kiely, A. Ansar, A. Castano, M. Klimesh, J. Maki, in The Interplanetary Network Progress Report, vol. 42168 (2007), pp. 1-27

S. Kumar, A. Kumar Rai, S.B. Rai, D.K. Rai, A.N. Singh, V.B. Singh, J. Mol. Struct. (2006). https://doi.org/ 10.1016/j.molstruc.2006.01.004

N.L. Lanza, A.M. Ollila, A. Cousin, R.C. Wiens, S. Clegg, N. Mangold, N. Bridges, D. Cooper, M. Schmidt, J. Berger, R. Arvidson, N. Melikechi, H.E. Newsom, R. Tokar, C. Hardgrove, A. Mezzacappa, R.S. Jackson, B. Clark, O. Forni, S.N. Maurice, A. Marion, B. Ryan, J. Blank, M. Deans, D. Delapp, R. Léveillé, R. McInroy, R. Martinez, P.-Y. Meslin, P. Pinet, Icarus (2015). https://doi.org/10.1016/j.icarus. 2014.05.038

N.L. Lanza et al., Geophys. Res. Lett. (2016). https://doi.org/10.1002/2016GL069109

N.L. Lanza, B. Chide, S.M. Clegg, E. Dauson, O. Forni, C. Larmat, A.M. Ollila, A. Reyes-Newell, J. Ten Cate, R.C. Wiens, S. Maurice, in 51st LPSC, 16-20 March, 2020 at the Woodlands, Texas. LPI Contribution No. 2326 (2020), id. 2807

P. Larkin, Infrared and Raman Spectroscopy: Principles and Spectral Interpretation (Elsevier, Amsterdam, 2011), ISBN 978-0-12-386984-5

J. Lasue, A. Cousin, P.-Y. Meslin, N. Mangold, R.C. Wiens, G. Berger, E. Dehouck, O. Forni, W. Goetz, O. Gasnault, W. Rapin, S. Schroeder, A. Ollila, J. Johnson, S. Le Mouélic, S. Maurice, R. Anderson, D. Blaney, B. Clark, S.M. Clegg, C. d'Uston, C. Fabre, N. Lanza, M.B. Madsen, J. Martin-Torres, N. Melikechi, H. Newsom, V. Sautter, M.P. Zorzano, Geophys. Res. Lett. (2018). https://doi.org/10.1029/ 2018GL079210

S. Le Mouélic, O. Gasnault, K.E. Herkenhoff, N.T. Bridges, Y. Langevin, N. Mangold, S. Maurice, R.C. Wiens, P. Pinet, H.E. Newsom, R.G. Deen, J.F. Bell, J.R. Johnson, W. Rapin, B. Barraclough, D.L. Blaney, L. Deflores, J. Maki, M.C. Malin, R. Pérez, M. Saccoccio, Icarus (2015). https://doi.org/10. 1016/j.icarus.2014.05.030

J. L'Haridon, N. Mangold, A.A. Fraeman, J.R. Johnson, A. Cousin, W. Rapin, G. David, E. Dehouck, V. Sun, J. Frydenvang, O. Gasnault, P. Gasda, N. Lanza, O. Forni, P.-Y. Meslin, S.P. Schwenzer, J. Bridges, B. Horgan, C.H. House, M. Salvatore, S. Maurice, R.C. Wiens, J. Geophys. Res., Planets (2020). https:// doi.org/10.1029/2019JE006299

V.M. Lisitsyn, E.F. Polisadova, D.T. Valiev, O.V. Pavlov, J. Appl. Spectrosc. (2011). https://doi.org/10.1007/ s10812-011-9482-1

L. Liu, L. Deng, L. Fan, X. Huang, Y. Lu, W. Shen, L. Jiang, J.-F. Silvain, Y. Lu, Opt. Express (2017). https:// doi.org/10.1364/OE.25.027000

M.C. Malin, M.A. Ravine, M.A. Caplinger, F. Tony Ghaemi, J.A. Schaffner, J.N. Maki, J.F. Bell, J.F. Cameron, W.E. Dietrich, K.S. Edgett, L.J. Edwards, J.B. Garvin, B. Hallet, K.E. Herkenhoff, E. Heydari, L.C. Kah, M.T. Lemmon, M.E. Minitti, T.S. Olson, T.J. Parker, S.K. Rowland, J. Schieber, R. Sletten, R.J. Sullivan, D.Y. Sumner, R. Aileen Yingst, B.M. Duston, S. McNair, E.H. Jensen, Earth Space Sci. (2017). https://doi.org/10.1002/2016EA000252

N. Mangold, L.M. Thompson, O. Forni, A.J. Williams, C. Fabre, L. Le Deit, R.C. Wiens, R. Williams, R.B. Anderson, D.L. Blaney, F. Calef, A. Cousin, S.M. Clegg, G. Dromart, W.E. Dietrich, K.S. Edgett, M.R. Fisk, O. Gasnault, R. Gellert, J.P. Grotzinger, L. Kah, S. Le Mouélic, S.M. McLennan, S. Maurice, P.Y. Meslin, H.E. Newsom, M.C. Palucis, W. Rapin, V. Sautter, K.L. Siebach, K. Stack, D. Sumner, A. Yingst, J. Geophys. Res., Planets (2016). https://doi.org/10.1002/2015JE004977

N. Mangold, M.E. Schmidt, M.R. Fisk, O. Forni, S.M. McLennan, D.W. Ming, V. Sautter, D. Sumner, A.J. Williams, S.M. Clegg, A. Cousin, O. Gasnault, R. Gellert, J.P. Grotzinger, R.C. Wiens, Icarus (2017). https://doi.org/10.1016/j.icarus.2016.11.005

R. Mason, M. Clouter, R. Goulding, Phys. Chem. Miner. (2005). https://doi.org/10.1007/s00269-005-0004-7

S. Maurice, R.C. Wiens, M. Saccoccio, B. Barraclough, O. Gasnault, O. Forni, N. Mangold, D. Baratoux, S. Bender, G. Berger, J. Bernardin, M. Berthé, N. Bridges, D. Blaney, M. Bouyé, P. Caïs, B. Clark, S. Clegg, A. Cousin, D. Cremers, A. Cros, L. DeFlores, C. Derycke, B. Dingler, G. Dromart, B. Dubois, M. Dupieux, E. Durand, L. d'Uston, C. Fabre, B. Faure, A. Gaboriaud, T. Gharsa, K. Herkenhoff, E. Kan, L. Kirkland, D. Kouach, J.-L. Lacour, Y. Langevin, J. Lasue, S. Le Mouélic, M. Lescure, E. Lewin, D. Limonadi, G. Manhès, P. Mauchien, C. McKay, P.-Y. Meslin, Y. Michel, E. Miller, H.E. Newsom, G. Orttner, A. Paillet, L. Parès, Y. Parot, R. Pérez, P. Pinet, F. Poitrasson, B. Quertier, B. Sallé, C. Sotin, V. Sautter, H. Séran, J.J. Simmonds, J.-B. Sirven, R. Stiglich, N. Striebig, J.-J. Thocaven, M.J. Toplis, D. Vaniman, Space Sci. Rev. (2012). https://doi.org/10.1007/s11214-012-9912-2 
S. Maurice, S.M. Clegg, R.C. Wiens, O. Gasnault, W. Rapin, O. Forni, A. Cousin, V. Sautter, N. Mangold, L. Le Deit, M. Nachon, R.B. Anderson, N.L. Lanza, C. Fabre, V. Payré, J. Lasue, P.-Y. Meslin, R.J. Léveillé, B.L. Barraclough, P. Beck, S.C. Bender, G. Berger, J.C. Bridges, N.T. Bridges, G. Dromart, M.D. Dyar, R. Francis, J. Frydenvang, B. Gondet, B.L. Ehlmann, K.E. Herkenhoff, J.R. Johnson, Y. Langevin, M.B. Madsen, N. Melikechi, J.-L. Lacour, S. Le Mouélic, E. Lewin, H.E. Newsom, A.M. Ollila, P. Pinet, S. Schröder, J.-B. Sirven, R.L. Tokar, M.J. Toplis, C. d'Uston, D.T. Vaniman, A.R. Vasavada, J. Anal. At. Spectrom. (2016). https://doi.org/10.1039/C5JA00417A

T.H. McConnochie, M.D. Smith, M.J. Wolff, S. Bender, M. Lemmon, R.C. Wiens, S. Maurice, O. Gasnault, J. Lasue, P.-Y. Meslin, A.-M. Harri, M. Genzer, O. Kemppinen, G.M. Martínez, L. DeFlores, D. Blaney, J.R. Johnson, J.F. Bell, Icarus (2018). https://doi.org/10.1016/j.icarus.2017.10.043

P.-Y. Meslin, O. Gasnault, O. Forni, S. Schröder, S. Clegg, G. Berger, J. Lasue, A. Cousin, S. Le Mouelic, S. Maurice, N. Mangold, C. Fabre, R. Wiens, B. Ehlmann, N. Lanza, P. Pinet, R. Anderson, D. Archer, D. Bish, D. Blake, D. Blaney, N. Bridges, B. Clark, G. Dormart, M.D. Dyar, M. Fisk, W. Goetz, K. Herkenhoff, J.-L. Lacour, Y. Langevin, L. Leshin, R. Leveille, E. Lewin, M.B. Madsen, T.H. McConnochie, J.E. Moores, H. Newsom, A. Ollila, R. Perez, E. Rampe, N. Renno, V. Sautter, J.-B. Sirven, M. de la Torre, L. d'Uston, D. Vaniman (the MSL Science Team), Science (2013). https:// doi.org/10.1126/science. 1238670

A. Meurisse, J. Carpenter, Planet. Space Sci. (2020). https://doi.org/10.1016/j.pss.2020.104853

A.K. Misra, T.E. Acosta-Maeda, S.K. Sharma, C.P. McKay, P.J. Gasda, G.J. Taylor, P.G. Lucey, L. Flynn, M. Nurul Abedin, S.M. Clegg, R. Wiens, Astrobiology (2016). https://doi.org/10.1089/ast.2015.1400

A. Miziolek, V. Palleschi, I. Schechter, Laser Induced Breakdown Spectroscopy (Cambridge University Press, Cambridge, 2006). ISBN 9780521852746

F. Montmessin, O. Korablev, F. Lefèvre, J.-L. Bertaux, A. Fedorova, A. Trokhimovskiy, J.Y. Chaufray, G. Lacombe, A. Reberac, L. Maltagliati, Y. Willame, S. Guslyakova, J.-C. Gérard, A. Stiepen, D. Fussen, N. Mateshvili, A. Määttänen, F. Forget, O. Witasse, F. Leblanc, A.C. Vandaele, E. Marcq, B. Sandel, B. Gondet, N. Schneider, M. Chaffin, N. Chapron, Icarus (2017). https://doi.org/10.1016/j.icarus.2017.06. 022

S. Murchie, R. Arvidson, P. Bedini, K. Beisser, J.-P. Bibring, J. Bishop, J. Boldt, P. Cavender, T. Choo, R.T. Clancy, E.H. Darlington, D. Des Marais, R. Espiritu, D. Fort, R. Green, E. Guinness, J. Hayes, C. Hash, K. Heffernan, J. Hemmler, G. Heyler, D. Humm, J. Hutcheson, N. Izenberg, R. Lee, J. Lees, D. Lohr, E. Malaret, T. Martin, J.A. McGovern, P. McGuire, R. Morris, J. Mustard, S. Pelkey, E. Rhodes, M. Robinson, T. Roush, E. Schaefer, G. Seagrave, F. Seelos, P. Silverglate, S. Slavney, M. Smith, W.-J. Shyong, K. Strohbehn, H. Taylor, P. Thompson, B. Tossman, M. Wirzburger, M. Wolff, J. Geophys. Res. (2007). https://doi.org/10.1029/2006JE002682

S.L. Murchie, J.F. Mustard, B.L. Ehlmann, R.E. Milliken, J.L. Bishop, N.K. McKeown, E.Z. Noe Dobrea, F.P. Seelos, D.L. Buczkowski, S.M. Wiseman, R.E. Arvidson, J.J. Wray, G. Swayze, R.N. Clark, D.J. Des Marais, A.S. McEwen, J.-P. Bibring, J. Geophys. Res., Planets (2009). https://doi.org/10.1029/ 2009JE003342

N. Murdoch, B. Chide, J. Lasue, A. Cadu, A. Sournac, M. Bassas-Portús, X. Jacob, J. Merrison, J.J. Iversen, C. Moretto, C. Velasco, L. Parès, A. Hynes, V. Godiver, R.D. Lorenz, P. Cais, P. Bernardi, S. Maurice, R.C. Wiens, D. Mimoun, Planet. Space Sci. (2019). https://doi.org/10.1016/j.pss.2018.09.009

J.F. Mustard, M. Adler, A. Allwood, D.S. Bass, D.W. Beaty, J.F. Bell III, W.B. Brinckerhoff, M. Carr, D.J. Des Marais, B. Drake, K.S. Edgett, J. Eigenbrode, L.T. Elkins-Tanton, J.A. Grant, S.M. Milkovich, D. Ming, C. Moore, S. Murchie, T.C. Onstott, S.W. Ruff, M.A. Sephton, A. Steele, A. Treiman, Report of the Mars 2020 Science Definition Team (Mars Exploration Program Analysis Group, 2013). http:// mepag.jpl.nasa.gov/reports/MEP/Mars_2020_SDT_Report_Final.pdf

M. Nachon, S.M. Clegg, N. Mangold, S. Schröder, L.C. Kah, G. Dromart, A. Ollila, J.R. Johnson, D.Z. Oehler, J.C. Bridges, S. Le Mouélic, O. Forni, R.C. Wiens, R.B. Anderson, D.L. Blaney, J.F. Bell, B. Clark, A. Cousin, M.D. Dyar, B. Ehlmann, C. Fabre, O. Gasnault, J. Grotzinger, J. Lasue, E. Lewin, R. Léveillé, S. McLennan, S. Maurice, P.-Y. Meslin, W. Rapin, M. Rice, S.W. Squyres, K. Stack, D.Y. Sumner, D. Vaniman, D. Wellington, J. Geophys. Res., Planets (2014). https://doi.org/10.1002/ 2013JE004588

H. Nair, M. Allen, A.D. Anbar, Y.L. Yung, R.T. Clancy, Icarus (1994). https://doi.org/10.1006/icar.1994.1137

D.E. Northup et al., J. Geophys. Res. (2010). https://doi.org/10.1029/2009JG001107

A.M. Ollila, H.E. Newsom, B. Clark III, R.C. Wiens, A. Cousin, J.G. Blank, N. Mangold, V. Sautter, S. Maurice, S.M. Clegg, O. Gasnault, O. Forni, R. Tokar, E. Lewin, M.D. Dyar, J. Lasue, R. Anderson, S.M. McLennan, J. Bridges, D. Vaniman, N. Lanza, C. Fabre, N. Melikechi, G.M. Perrett, J.L. Campbell, P.L. King, B. Barraclough, D. Delapp, S. Johnstone, P.-Y. Meslin, A. Rosen-Gooding, J. Williams (The MSL Science Team), J. Geophys. Res., Planets (2014). https://doi.org/10.1002/2013JE004517

K.J. Parchert et al., Geomicrobiology (2012). https://doi.org/10.1080/01490451.2011.619636

J.D. Pasteris, O. Beyssac, Elements (2020). https://doi.org/10.2138/gselements.16.2.87 
V. Payré, C. Fabre, A. Cousin, V. Sautter, R.C. Wiens, O. Forni, O. Gasnault, N. Mangold, P.-Y. Meslin, J. Lasue, A. Ollila, W. Rapin, S. Maurice, M. Nachon, L. Le Deit, N. Lanza, S. Clegg, Alkali trace elements in Gale crater, Mars, with ChemCam: calibration update and geological implications. J. Geophys. Res., Planets (2017). https://doi.org/10.1002/2016JE005201

L. Peret, O. Gasnault, R. Dingler, Y. Langevin, S. Bender, D. Blaney, S. Clegg, C. Clewans, D. Delapp, C. Donny, S. Johnstone, C. Little, E. Lorigny, R. McInroy, S. Maurice, N. Mittal, B. Pavri, R. Perez, R.C. Wiens, C. Yana, in 14th International Conference on Space Operations (2016). https://doi.org/10.2514/ 6.2016-2539

W. Rapin, P.-Y. Meslin, S. Maurice, D. Vaniman, M. Nachon, N. Mangold, S. Schröder, O. Gasnault, O. Forni, R.C. Wiens, G.M. Martínez, A. Cousin, V. Sautter, J. Lasue, E.B. Rampe, D. Archer, Earth Planet. Sci. Lett. (2016). https://doi.org/10.1016/j.epsl.2016.07.045

W. Rapin, P.-Y. Meslin, S. Maurice, R.C. Wiens, D. Laporte, B. Chauviré, O. Gasnault, S. Schröder, P. Beck, S. Bender, O. Beyssac, A. Cousin, E. Dehouck, C. Drouet, O. Forni, M. Nachon, N. Melikechi, B. Rondeau, N. Mangold, N.H. Thomas, Quantification of water content by laser induced breakdown spectroscopy on Mars. Spectrochim. Acta, Part B, At. Spectrosc. (2017). https://doi.org/10.1016/j.sab.2017. 02.007

P. Rösch, M. Harz, M. Schmitt, K.-D. Peschke, O. Ronneberger, H. Burkhardt, H.-W. Motzkus, M. Lankers, S. Hofer, H. Thiele, J. Popp, Appl. Environ. Microbiol. (2005). https://doi.org/10.1128/AEM.71.3.16261637.2005

C. Royer, F. Poulet, J.-M. Reess, C. Pilorget, V. Hamm, T. Fouchet, S. Maurice, O. Forni, P. Bernardi, F. Montmessin, L. Lapauw, J. Parisot, M. Bonafous, O. Gasnault, R.C. Wiens, Rev. Sci. Instrum. (2020). https://doi.org/10.1063/1.5145390

F. Rull, S. Maurice, I. Hutchinson, A. Moral, C. Perez, C. Diaz, M. Colombo, T. Belenguer, G. Lopez-Reyes, A. Sansano, O. Forni, Y. Parot, N. Striebig, S. Woodward, C. Howe, N. Tarcea, P. Rodriguez, L. Seoane, A. Santiago, J.A. Rodriguez-Prieto, J. Medina, P. Gallego, R. Canchal, P. Santamaría, G. Ramos, J.L. Vago (RLS Team), Astrobiology (2017). https://doi.org/10.1089/ast.2016.1567

F. Rull, C. Ryan, T. Säuberlich, F. Schrandt, S. Ulamec, T. Usui, R. Vance, in 51st Lunar and Planetary Science Conference (2020). Contribution No. 2326

B. Sallé, J.-L. Lacour, P. Mauchien, P. Fichet, S. Maurice, G. Manhès, Spectrochim. Acta (2006). https:// doi.org/10.1016/j.sab.2006.02.003

V. Sautter, M. Toplis, R. Wiens, A. Cousin, C. Fabre, O. Gasnault, S. Maurice, O. Forni, J. Lasue, A. Ollila, J. Bridges, N. Mangold, S. Le Mouélic, M. Fisk, P.-Y. Meslin, P. Beck, P. Pinet, L. Le Deit, W. Rapin, E. Stolper, H. Newsom, D. Dyar, N. Lanza, D. Vaniman, S. Clegg, J. Wray, Nat. Geosci. (2015). https:// doi.org/10.1038/ngeo2474

V. Sautter, M.J. Toplis, P. Beck, N. Mangold, R. Wiens, P. Pinet, A. Cousin, S. Maurice, L. LeDeit, R. Hewins, O. Gasnault, C. Quantin, O. Forni, H. Newsom, P.-Y. Meslin, J. Wray, N. Bridges, V. Payré, W. Rapin, S. Le Mouélic, Lithos (2016). https://doi.org/10.1016/j.lithos.2016.02.023

S. Schröder, P.-Y. Meslin, O. Gasnault, S. Maurice, A. Cousin, R.C. Wiens, W. Rapin, M.D. Dyar, N. Mangold, O. Forni, M. Nachon, S. Clegg, J.R. Johnson, J. Lasue, S. Le Mouélic, A. Ollila, P. Pinet, V. Sautter, D. Vaniman, Icarus (2015). https://doi.org/10.1016/j.icarus.2014.08.029

S. Schröder, T. Belenguer, U. Böttger, M. Buder, Y. Cho, E. Dietz, M. Gensch, T. Hagelschuer, F. Hanke, H.-W. Hübers, S. Kameda, E. Kopp, S. Kubitza, A. Moral, C. Paproth, M. Pertenais, G. Peter, K. Rammelkamp, P. Rodriguez

S.K. Sharma, M.J. Egan, in Remote Compositional Analysis: Techniques for Understanding Spectroscopy, Mineralogy, and Geochemistry of Planetary Surfaces (2019). https://doi.org/10.1017/9781316888872. 008

J.-L. Starck, J. Fadili, F. Murtagh, IEEE Trans. Image Process. (2007). https://doi.org/10.1109/TIP.2006. 887733

G. Taylor, Proc. R. Soc. Lond. Ser. A (1950). https://doi.org/10.1098/rspa.1950.0049

C.E. Viviano-Beck, F.P. Seelos, S.L. Murchie, E.G. Kahn, K.D. Seelos, H.W. Taylor, K. Taylor, B.L. Ehlmann, S.M. Wisemann, J.F. Mustard, M.F. Morgan, J. Geophys. Res., Planets (2014). https://doi.org/10.1002/ 2014JE004627

D.S. Vogt, K. Rammelkamp, S. Schröder, H.-W. Hübers, Icarus (2018). https://doi.org/10.1016/j.icarus.2017. 12.006

R.C. Wiens, S. Maurice (MSL Science Team), Elements (2015). https://doi.org/10.2113/gselements.11.1.33

R.C. Wiens, S. Maurice, B. Barraclough, M. Saccoccio, W.C. Barkley, J.F. Bell, S. Bender, J. Bernardin, D. Blaney, J. Blank, M. Bouyé, N. Bridges, N. Bultman, P. Caïs, R.C. Clanton, B. Clark, S. Clegg, A. Cousin, D. Cremers, A. Cros, L. DeFlores, D. Delapp, R. Dingler, C. D’Uston, M. Darby Dyar, T. Elliott, D. Enemark, C. Fabre, M. Flores, O. Forni, O. Gasnault, T. Hale, C. Hays, K. Herkenhoff, E. Kan, L. Kirkland, D. Kouach, D. Landis, Y. Langevin, N. Lanza, F. LaRocca, J. Lasue, J. Latino, D. Limonadi, C. Lindensmith, C. Little, N. Mangold, G. Manhes, P. Mauchien, C. McKay, E. Miller, J. Mooney, 
R.V. Morris, L. Morrison, T. Nelson, H. Newsom, A. Ollila, M. Ott, L. Pares, R. Perez, F. Poitrasson, C. Provost, J.W. Reiter, T. Roberts, F. Romero, V. Sautter, S. Salazar, J.J. Simmonds, R. Stiglich, S. Storms, N. Striebig, J.-J. Thocaven, T. Trujillo, M. Ulibarri, D. Vaniman, N. Warner, R. Waterbury, R. Whitaker, J. Witt, B. Wong-Swanson, Space Sci. Rev. (2012). https://doi.org/10.1007/s11214-012-9902-4

R.C. Wiens, S. Maurice, J. Lasue, O. Forni, R.B. Anderson, S. Clegg, S. Bender, B.L. Barraclough, L. Deflores, D. Blaney, R. Perez, N. Lanza, A. Ollila, A. Cousin, O. Gasnault, D. Vaniman, M.D. Dyar, C. Fabre, V. Sautter, D. Delapp, H. Newsom, N. Melikechi (the ChemCam Team), Spectrochim. Acta B (2013). https://doi.org/10.1016/j.sab.2013.02.003

J.-P. Williams, J. Geophys. Res. (2001). https://doi.org/10.1029/1999JE001174

R.M.E. Williams et al. (MSL Science Team), Science (2013). https://doi.org/10.1126/science.1237317

R. Xin, C. Tingni, L. Dawei, L. Jianjun, Z. Hongbo, F. Qiang, Z. Zhoubin, X. Weimin, in European Planetary Science Congress (2018), EPSC2018-759

F. Zapata, C. García-Ruiz, Spectrochim. Acta, Part A, Mol. Biomol. Spectrosc. (2018). https://doi.org/10. 1016/j.saa.2017.08.058

Y.-H. Zhang, C.K. Chan, J. Phys. Chem. A (2003). https://doi.org/10.1021/jp0271256 